\title{
Concise Enantioselective Total Synthesis of Cardiotonic Steroids 19-Hydroxysarmentogenin and Trewianin Aglycone.
}

Will Kaplan, Dr. Hem Raj Khatri, and Dr. Pavel Nagorny

Department of Chemistry, University of Michigan, 930 N. University Avenue, Ann Arbor, MI, 48109

\section{Contents}

1. Nomenclature.

Page S2

2. Experimental procedures and spectral tabulations
a. Synthesis of Tetra-ol 4
.Pages S4 -S8
b. Synthesis of 19-Hydroxysarmentogenin (2)
Pages S8 - S15
c. Synthesis of Panogenin core (17).
Pages S16 - S17
d. Synthesis of Trewianin aglycone (3)
Pages S18 - S27

3. Structural Proof.

Pages S28 - S32

4. NMR spectra.

Pages S33 - S120

5. References

Page S121 


\section{Nomenclature}

The standard numbering and facial referencing convention for cardiotonic steroids (shown below) is used throughout the supporting information and for all intermediates.
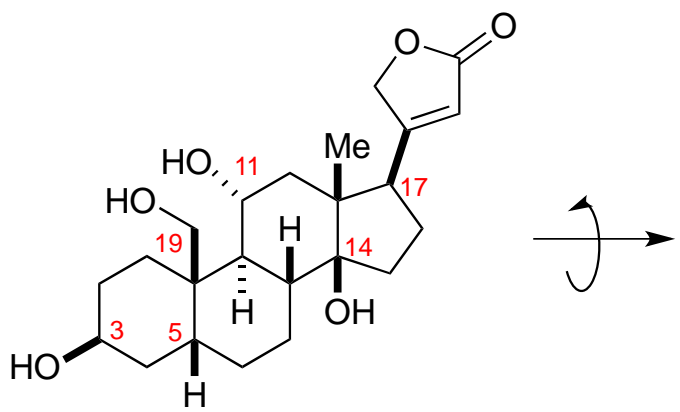
19-hydroxysarmentogenin (2)

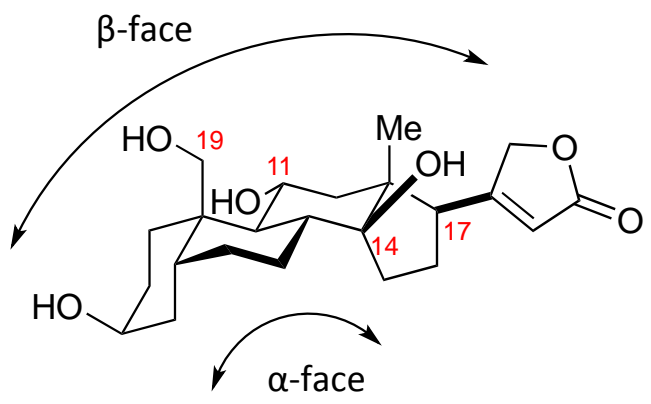




\section{Experimental}

General Methods: All reagents and solvents were purchased from commercial sources and were used as received without further purification unless otherwise specified. THF and DMF were purified by Innovative Technology's Pure-Solve System using basic alumina. All reactions were carried out under a positive pressure of nitrogen in flame- or oven-dried glassware with magnetic stirring. Reactions were cooled using a cryocool or external cooling baths (sodium chloride/ice water $\left(-10^{\circ} \mathrm{C}\right)$ or dry ice/acetone $\left(-78^{\circ} \mathrm{C}\right)$ ). Heating was achieved by use of a silicone oil bath with heating controlled by electronic contact thermometer. Deionized water was used in the preparation of all aqueous solutions and for all aqueous extractions. Solvents used for extraction and chromatography were ACS or HPLC grade. Purification of reactions mixtures was performed by flash chromatography using SiliCycle SiliaFlash P60 (230-400 mesh). Alternatively, purifications were achieved using CombiFlash Rf+ Lumen Automated Flash Chromatography System with UV and ELSD detector using either SiliCycle SiliaFlash P60 (230-400 mesh) or Teledyne RediSep Rf Gold Silica (20-40 $\mu \mathrm{m}$ spherical). Yields indicate the isolated yield of the title compound with $\geq 95 \%$ purity as determined by ${ }^{1} \mathrm{H}$ NMR analysis. Whereas the yields in the article are the average yields of two or more experiments, the yields in the supporting information describe the result of a single experiment. Diastereomeric ratios were determined by ${ }^{1} \mathrm{H}$ NMR analysis. Enantiomeric excess was determined by HPLC analysis using a Waters e2695 Separations Module with a Waters 2998 photodiode array detector on a Chiralcel AD-H column. ${ }^{1} \mathrm{H}$ NMR spectra were recorded on a Varian vnmrs $700(700 \mathrm{MHz})$, Varian vnmrs $500(500 \mathrm{MHz})$, Varian vnmrs $400(400 \mathrm{MHz})$, or a Varian Inova 500 $(500 \mathrm{MHz})$ spectrometer and chemical shifts $(\delta)$ are reported in parts per million (ppm) with solvent resonance as the internal standard $\left(\mathrm{CDCl}_{3}\right.$ at $\delta 7.26, \mathrm{D}_{3} \mathrm{COD}$ at $\left.\delta 3.31\right)$. Tabulated ${ }^{1} \mathrm{H}$ NMR Data are reported as $\mathrm{s}=$ singlet, $\mathrm{d}=$ doublet, $\mathrm{t}=$ triplet, $\mathrm{q}=$ quartet, $\mathrm{qn}=$ quintet, sext $=$ sextet, $\mathrm{m}=$ multiplet, ovrlp = overlap, and coupling constants in $\mathrm{Hz}$. Proton-decoupled ${ }^{13} \mathrm{C}$ NMR spectra were recorded on Varian vnmrs $700(700 \mathrm{MHz})$ spectrometer and chemical shifts $(\delta)$ are reported in ppm with solvent resonance as the internal standard $\left(\mathrm{CDCl}_{3}\right.$ at $\delta 77.0, \mathrm{D}_{3} \mathrm{COD}$ at $\left.\delta 49.0\right)$. High resolution mass spectra (HRMS) were performed and recorded on Micromass AutoSpec Ultima or VG (Micromass) 70-250-S Magnetic sector mass spectrometers in the University of Michigan mass spectrometry laboratory. Infrared (IR) spectra were recorded as thin films a Perkin Elmer Spectrum BX FT-IR spectrometer. Absorption peaks are reported in wavenumbers $\left(\mathrm{cm}^{-1}\right)$. Optical rotations were measured at room temperature in $\mathrm{CHCl}_{3}$ or $\mathrm{H}_{3} \mathrm{COH}$ on a Jasco P-2000 polarimeter. 

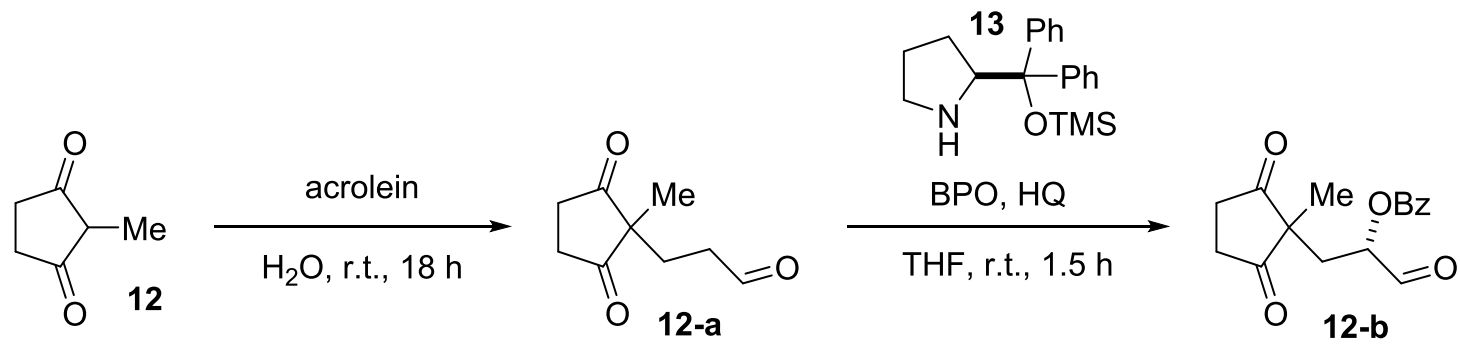

Compound 12-b. Diketone 12 (20.0 g, $178.4 \mathrm{mmol})$ was suspended in $\mathrm{H}_{2} \mathrm{O}(336 \mathrm{~mL})$. Acrolein $(15.0 \mathrm{~g}$, $17.8 \mathrm{~mL}, 267.5 \mathrm{mmol}$ ) was then added. The reaction was allowed to stir at room temperature overnight to provide a homogeneous solution. The reaction was then extracted 6-7x with $\mathrm{CH}_{2} \mathrm{Cl}_{2}(200 \mathrm{~mL})$ and the organic layers were combined, dried over $\mathrm{Na}_{2} \mathrm{SO}_{4}$, filtered, and concentrated to afford compound 12-a $(29.0 \mathrm{~g}, 172.6 \mathrm{mmol})$ as a colorless oil in $97 \%$ yield without the need for purification. The time sensitive aldehyde is then used immediately for the next step: Benzoyl peroxide $(18.7 \mathrm{~g}, 77.4 \mathrm{mmol})$ and hydroquinone (990 mg, $9 \mathrm{mmol}$ ) were solvated in THF $(300 \mathrm{~mL})$ and $\mathrm{H}_{2} \mathrm{O}(0.5 \mathrm{~mL})$. Then, (S)-2(diphenyl((trimethylsilyl)oxy)methyl)pyrrolidine 13 (1.95 g, $6.0 \mathrm{mmol})$ was added followed by aldehyde 12-a $(10.0 \mathrm{~g}, 59.5 \mathrm{mmol})$. The reaction mixture was then stirred at room temperature under $\mathrm{N}_{2}$ until the disappearance of starting material by ${ }^{1} \mathrm{H}$ NMR. After $1.5 \mathrm{~h}$, the reaction mixture was concentrated in vacuo and directly purified using flash column chromatography with 30\% EtOAc in hexanes to afford title compound 12-b (13.5 g, $46.8 \mathrm{mmol}$ ) as viscous oil in $79 \%$ yield. The material was immediately used for the next step without further purification.
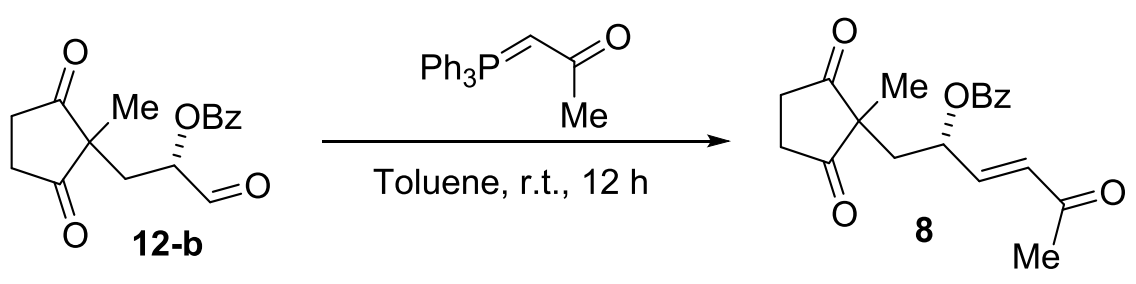

Compound 8. Aldehyde 12-b (13.5 g, $46.8 \mathrm{mmol})$ was solvated in toluene ( $300 \mathrm{~mL}$ ) and Wittig reagent 1(tripheylphosphoranylidene)-2-propanone $(22.2 \mathrm{~g}, 69.7 \mathrm{mmol}$ ) was added. The reaction was allowed to stir at room temperature for $12 \mathrm{~h}$. The solution was then concentrated in vacuo and directly purified by flash column chromatography with $20 \% \rightarrow 30 \%$ EtOAc in hexanes to afford title compound 8 ( $11.5 \mathrm{~g}$, $35.0 \mathrm{mmol}$ ) as a white solid in 75\% yield, 95\% ee (Chiralpak AD-H, 12\% iPrOH in hexanes, R-enantiomer 13.2 minutes, S-enantiomer 28 minutes), $\mathrm{R}_{\mathrm{f}}=0.3$ (33\% EtOAc in Hexanes). ${ }^{1} \mathbf{H}$ NMR $\left(700 \mathrm{MHz}, \mathrm{CDCl}_{3}\right)$ : $7.61(\mathrm{t}, J=7.4 \mathrm{~Hz}, 1 \mathrm{H}), 7.48(\mathrm{t}, J=7.8 \mathrm{~Hz}, 2 \mathrm{H}), 6.64(\mathrm{dd}, J=16.1,5.3 \mathrm{~Hz}, 1 \mathrm{H}), 6.19$ (dd, $J=16.1,1.2 \mathrm{~Hz}$, $1 \mathrm{H}), 5.63-5.49(\mathrm{~m}, 1 \mathrm{H}), 2.82-2.69(\mathrm{~m}, 2 \mathrm{H}), 2.57-2.49(\mathrm{~m}, 1 \mathrm{H}), 2.49-2.36(\mathrm{~m}, 2 \mathrm{H}), 2.25(\mathrm{~s}, 3 \mathrm{H}), 2.15$ (dd, $J=14.6,3.3 \mathrm{~Hz}, 1 \mathrm{H}), 1.19(\mathrm{~s}, 3 \mathrm{H}) .{ }^{13} \mathrm{C}$ NMR $\left(175 \mathrm{MHz}, \mathrm{CDCl}_{3}\right)$ : $\delta$ 215.7, 214.8, 197.6, 165.4, 142.7, 133.8, 130.6, 129.7, 128.7, 128.7, 70.1, 54.3, 37.9, 34.7, 34.6, 27.4, 23.1. HRMS (ESI): Calculated for $\mathrm{C}_{19} \mathrm{H}_{20} \mathrm{O}_{5} \mathrm{Na}^{+}[\mathrm{M}+\mathrm{Na}]^{+}$351.1203, found 351.1206. IR (film, $\mathrm{cm}^{-1}$ ): 1722, 1678, 1364, 1252, 1100, 1070, $982,715 .[\alpha]_{D}^{25}=-29\left(\mathrm{c}=1.0, \mathrm{CHCl}_{3}\right)$. 

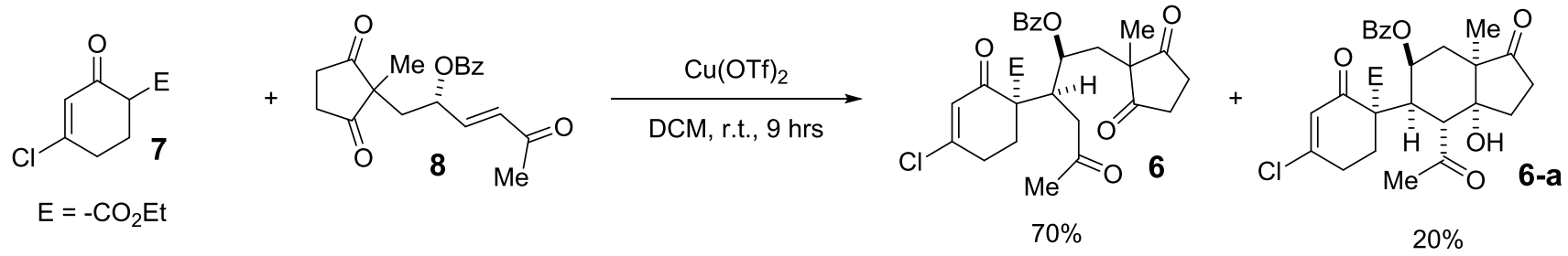

Compound 6. Enone 8 ( $5.7 \mathrm{~g}, 17.3 \mathrm{mmol})$, ketoester $^{1} 7(7.0 \mathrm{~g}, 34.5 \mathrm{mmol})$, and copper(II) triflate $(3.1 \mathrm{~g}$, $8.6 \mathrm{mmol}$ ) were added successively to a flame dried flask. The vessel was purged with $\mathrm{N}_{2}$ and $\mathrm{dry} \mathrm{CH}_{2} \mathrm{Cl}_{2}$ $(10 \mathrm{~mL})$ was added. The reaction mixture was allowed to stir at room temperature until enone 8 was completely consumed as determined by TLC. After $9 \mathrm{~h}$, the reaction mixture was filtered through a pad of silica gel and eluted with diethyl ether. The filtrate was concentrated and directly purified by flash column chromatography with $30 \% \rightarrow 50 \%$ EtOAc in hexanes to afford title compound 6 (6.4 g, 12.1 $\mathrm{mmol}$ ) as a white foam in $70 \%$ yield, $R_{f}=0.2$ (33\% EtOAc in Hexanes), and 6:1 d.r. as well as crude fractions of half cyclized aldol product 6-a $(1.8 \mathrm{~g}, 3.45 \mathrm{mmol})$ in $20 \%$ yield. Adduct 6: ${ }^{1} \mathbf{H}$ NMR $(500 \mathrm{MHz}$, $\left.\mathrm{CDCl}_{3}\right): \delta 7.89(\mathrm{~d}, J=7.2 \mathrm{~Hz}, 2 \mathrm{H}), 7.59(\mathrm{t}, J=7.4 \mathrm{~Hz}, 1 \mathrm{H}), 7.45(\mathrm{t}, J=7.7 \mathrm{~Hz}, 2 \mathrm{H}), 6.18(\mathrm{~d}, J=1.2 \mathrm{~Hz}, 1 \mathrm{H})$, $5.20(\mathrm{~d}, J=8.6 \mathrm{~Hz}, 1 \mathrm{H}), 3.90-3.79(\mathrm{~m}, 2 \mathrm{H}), 3.21(\mathrm{t}, J=4.8 \mathrm{~Hz}, 1 \mathrm{H}), 3.04(\mathrm{dd}, J=19.1,5.7 \mathrm{~Hz}, 1 \mathrm{H}), 2.84$ (dd, $J=19.1,4.2 \mathrm{~Hz}, 1 \mathrm{H}), 2.81-2.71(\mathrm{~m}$, ovrlp, 3H), $2.70-2.60(\mathrm{~m}, 1 \mathrm{H}), 2.55(\mathrm{dt}, J=19.5,4.6 \mathrm{~Hz}, 1 \mathrm{H})$, 2.48 - 2.36 (m, ovrlp, $2 \mathrm{H}$ ), $2.23(\mathrm{~s}, 3 \mathrm{H}), 2.20$ (d, $J=10.1 \mathrm{~Hz}, 1 \mathrm{H}$ ), 2.08 (dd, J = 14.9, $2.7 \mathrm{~Hz}, 1 \mathrm{H}$ ), 1.94 (ddd, J $=14.2,9.2,5.3 \mathrm{~Hz}, 1 \mathrm{H}), 1.11(\mathrm{~s}, 3 \mathrm{H}), 1.08(\mathrm{t}, J=7.1 \mathrm{~Hz}, 3 \mathrm{H}) .{ }^{13} \mathrm{C}$ NMR $\left(100 \mathrm{MHz}, \mathrm{CDCl}_{3}\right): \delta 215.0,214.8$, 206.0, 191.9, 169.0, 166.4, 156.8, 133.5, 129.5, 128.6, 127.8, 70.6, 61.9, 58.1, 55.6, 39.5, 39.23, 37.4, 34.19, 34.15, 31.6, 30.0, 27.3, 24.3, 13.7. HRMS (ESI): Calculated for $\mathrm{C}_{28} \mathrm{H}_{31} \mathrm{ClO}_{8} \mathrm{Na}^{+}[\mathrm{M}+\mathrm{Na}]^{+} 553.1600$,

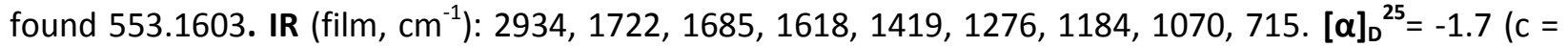
$\left.0.5, \mathrm{CHCl}_{3}\right)$.

Aldol product 6-a: ${ }^{1} \mathrm{H}$ NMR $\left(500 \mathrm{MHz}, \mathrm{CDCl}_{3}\right): \delta 7.87(\mathrm{~d}, J=7.4 \mathrm{~Hz}, 2 \mathrm{H}), 7.57(\mathrm{t}, J=7.4 \mathrm{~Hz}, 1 \mathrm{H}), 7.46(\mathrm{t}, J=$ $7.7 \mathrm{~Hz}, 2 \mathrm{H}), 6.07(\mathrm{~s}, 1 \mathrm{H}), 5.45(\mathrm{~d}, J=1.6 \mathrm{~Hz}, 1 \mathrm{H}), 4.31(\mathrm{~s}, 1 \mathrm{H}), 4.11(\mathrm{~d}, J=11.6 \mathrm{~Hz}, 1 \mathrm{H}), 3.78(\mathrm{dq}, J=14.3$, $7.1 \mathrm{~Hz}, 1 \mathrm{H}), 3.44(\mathrm{dq}, J=14.3,7.2 \mathrm{~Hz}, 1 \mathrm{H}), 2.92(\mathrm{~d}, J=11.6 \mathrm{~Hz}, 1 \mathrm{H}), 2.78-2.30(\mathrm{~m}, 10 \mathrm{H}), 2.22-2.00(\mathrm{~m}$, $1 \mathrm{H}), 1.90(\mathrm{dd}, J=13.5,8.8 \mathrm{~Hz}, 1 \mathrm{H}), 1.74(\mathrm{dd}, J=15.4,3.3 \mathrm{~Hz}, 1 \mathrm{H}), 1.02(\mathrm{~s}, 3 \mathrm{H}), 0.87(\mathrm{t}, J=7.1 \mathrm{~Hz}, 3 \mathrm{H}) .{ }^{13} \mathrm{C}$ NMR $\left(100 \mathrm{MHz}, \mathrm{CDCl}_{3}\right): \delta 217.3,214.5,193.2,168.5,165.3,154.1,133.3,129.7,129.5,128.4,126.3$, 78.0, 68.2, 61.4, 57.3, 51.5, 49.1, 38.6, 36.1, 34.6, 32.5, 31.0, 30.8, 28.5, 19.8, 13.5. HRMS (ESI): calculated for $\mathrm{C}_{28} \mathrm{H}_{32} \mathrm{ClO}_{8}{ }^{+} 531.1786[\mathrm{M}+\mathrm{H}]^{+}$, found 531.1773. IR (film, $\left.\mathrm{cm}^{-1}\right)$ : 3457(br), 2916, 1743, 1722, $1682,1620,1359,1271,1185,1072,1043,756,713 .[\alpha]_{D}^{25}=+91.9\left(\mathrm{c}=0.5, \mathrm{CHCl}_{3}\right)$.

Minor diastereomer of adduct 6 characteristic ${ }^{1} \mathrm{H}$ NMR peaks $\left(400 \mathrm{MHz}, \mathrm{CDCl}_{3}\right): \delta 7.88(\mathrm{~d}, J=7.4 \mathrm{~Hz}, 2 \mathrm{H})$, $7.59(\mathrm{t}, J=7.4 \mathrm{~Hz}, 1 \mathrm{H}), 7.45(\mathrm{t}, J=7.7 \mathrm{~Hz}, 2 \mathrm{H}), 6.09(\mathrm{~s}, 1 \mathrm{H}), 5.32(\mathrm{~d}, J=10.2 \mathrm{~Hz}, 1 \mathrm{H}), 4.16-3.95(\mathrm{~m}, 2 \mathrm{H})$, $3.24(\mathrm{t}, 1 \mathrm{H}), 2.99(\mathrm{dd}, \mathrm{J}=16.3,5.7 \mathrm{~Hz}, 1 \mathrm{H}), 2.23(\mathrm{~s}, 3 \mathrm{H}), 2.00-1.88(\mathrm{~m}, 1 \mathrm{H}), 1.13-1.04$ (ovrlp, 6H).
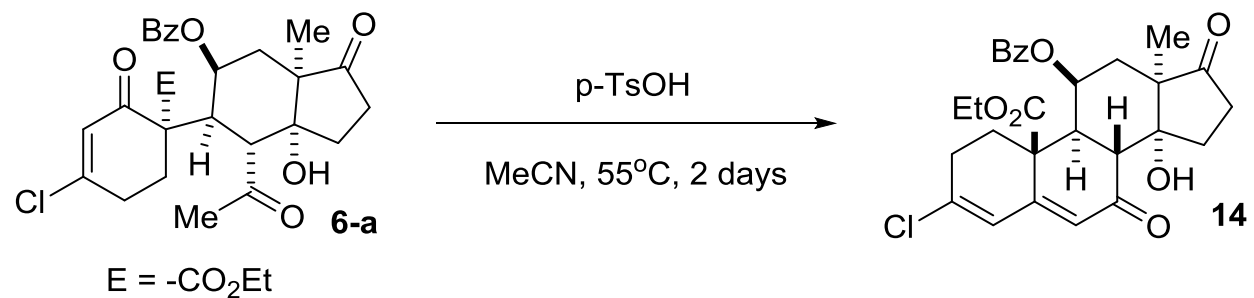

Compound 14. Crude fraction of half cyclized aldol product 6-a $(7.0 \mathrm{~g}, 13.2 \mathrm{mmol})$ and p-toluenesulfonic acid monohydrate $(26.0 \mathrm{~g}, 136.8 \mathrm{mmol})$ were solvated in acetonitrile $(350 \mathrm{~mL})$ and the solution was 
heated to $55^{\circ} \mathrm{C}$ for $48 \mathrm{~h}$. The reaction mixture was then concentrated and $\mathrm{CH}_{2} \mathrm{Cl}_{2}$ (400 mL) was added. The $\mathrm{p}-\mathrm{TsOH}$ precipitated and was filtered off. The filtrate was then concentrated and directly purified by flash column chromatography with $10 \% \rightarrow 30 \%$ EtOAc in hexanes to afford title compound 14 (3.8 g, 7.4 $\mathrm{mmol})$ as a white solid in $56 \%$ yield, $\mathrm{R}_{\mathrm{f}}=0.4$ (50\% EtOAc in Hexanes). ${ }^{1} \mathrm{H} \mathbf{N M R}\left(700 \mathrm{MHz}, \mathrm{CDCl}_{3}\right): \delta 7.80$ $(\mathrm{d}, J=8.3 \mathrm{~Hz}, 2 \mathrm{H}), 7.54(\mathrm{t}, J=7.4 \mathrm{~Hz}, 1 \mathrm{H}), 7.41(\mathrm{t}, J=7.8 \mathrm{~Hz}, 2 \mathrm{H}), 6.31(\mathrm{~d}, J=1.9 \mathrm{~Hz}, 1 \mathrm{H}), 5.95(\mathrm{~s}, 1 \mathrm{H}), 5.54$ $(\mathrm{s}, 1 \mathrm{H}), 3.68(\mathrm{dq}, J=10.9,7.1 \mathrm{~Hz}, 1 \mathrm{H}), 3.41(\mathrm{~d}, J=13.2 \mathrm{~Hz}, 1 \mathrm{H}), 3.32(\mathrm{dq}, J=10.9,7.1 \mathrm{~Hz}, 1 \mathrm{H}), 3.19(\mathrm{dd}, J=$ 13.0, $9.2 \mathrm{~Hz}, 1 \mathrm{H}$ ), 3.08 (s, 1H), $2.87-2.74$ (ovrlp, $2 \mathrm{H}$ ), 2.64 (dd, $J=15.1,3.6 \mathrm{~Hz}, 1 \mathrm{H}$ ), 2.56 - 2.44 (ovrlp, $3 \mathrm{H}), 2.39-2.29(\mathrm{~m}, 1 \mathrm{H}), 2.12(\mathrm{td}, J=12.5,9.7 \mathrm{~Hz}, 1 \mathrm{H}), 1.63(\mathrm{ddd}, J=13.0,11.9,6.1 \mathrm{~Hz}, 1 \mathrm{H}), 1.56(\mathrm{dd}, J=$ 15.1, $2.4 \mathrm{~Hz}, 1 \mathrm{H}), 1.00(\mathrm{~s}, 3 \mathrm{H}), 0.89(\mathrm{t}, J=7.1 \mathrm{~Hz}, 3 \mathrm{H}) .{ }^{13} \mathrm{C}$ NMR $\left(175 \mathrm{MHz}, \mathrm{CDCl}_{3}\right): \delta 216.5,200.8,169.6$, 164.8, 152.0, 144.2, 133.3, 129.8, 129.4, 128.3, 126.8, 125.3, 78.5, 66.8, 62.03, 52.1, 46.8, 46.4, 45.7, 34.8, 33.5, 32.1, 31.8, 30.3, 20.6, 13.6. HRMS (ESI): Calucalted for $\mathrm{C}_{28} \mathrm{H}_{30} \mathrm{ClO}_{7}^{+}[\mathrm{M}+\mathrm{H}]^{+} 513.1675$, found 513.1680. IR (film, cm ${ }^{-1}$ ): 3507(br), 2911, 1724, 1697, 1658, 1625, 1451, 1350, 1277, 1177, 1074, 1027, $900,717 .[\alpha]_{D}^{25}=-176\left(\mathrm{c}=1.1, \mathrm{CHCl}_{3}\right)$.
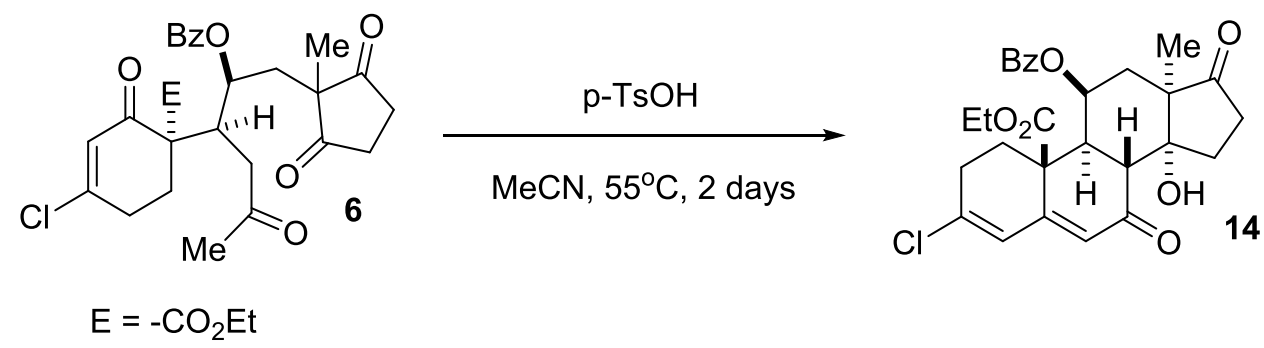

Compound 14. Michael adduct $6(8.0 \mathrm{~g}, 15.1 \mathrm{mmol})$ and p-toluenesulfonic acid monohydrate $(28.7 \mathrm{~g}$, $150.9 \mathrm{mmol}$ ) were solvated in acetonitrile $(400 \mathrm{~mL})$ and the solution was heated to $55^{\circ} \mathrm{C}$ for $48 \mathrm{~h}$. The reaction mixture was then concentrated and $\mathrm{CH}_{2} \mathrm{Cl}_{2}(500 \mathrm{~mL})$ was added. The $\mathrm{p}-\mathrm{TsOH}$ precipitated and was filtered off. The filtrate was then concentrated and directly purified by flash column chromatography with $10 \% \rightarrow 30 \%$ EtOAc in hexanes to afford title compound $\mathbf{1 4}(5.3 \mathrm{~g}, 10.4 \mathrm{mmol})$ as a white solid in 68\% yield, $\mathrm{R}_{\mathrm{f}}=0.4$ (50\% EtOAc in Hexanes). ${ }^{1} \mathrm{H}$ NMR $\left(700 \mathrm{MHz}, \mathrm{CDCl}_{3}\right): \delta 7.80(\mathrm{~d}, J=8.3 \mathrm{~Hz}$, $2 \mathrm{H}), 7.54(\mathrm{t}, J=7.4 \mathrm{~Hz}, 1 \mathrm{H}), 7.41(\mathrm{t}, J=7.8 \mathrm{~Hz}, 2 \mathrm{H}), 6.31(\mathrm{~d}, J=1.9 \mathrm{~Hz}, 1 \mathrm{H}), 5.54(\mathrm{~s}, 1 \mathrm{H}), 5.95(\mathrm{~s}, 1 \mathrm{H}), 3.68$ (dq, $J=10.9,7.1 \mathrm{~Hz}, 1 \mathrm{H}), 3.41(\mathrm{~d}, J=13.2 \mathrm{~Hz}, 1 \mathrm{H}), 3.32(\mathrm{dq}, J=10.9,7.1 \mathrm{~Hz}, 1 \mathrm{H}), 3.19$ (dd, $J=13.0,9.2$ $\mathrm{Hz}, 1 \mathrm{H}$ ), 3.08 (s, 1H), $2.74-2.87$ (ovrlp, $2 \mathrm{H}$ ), 2.64 (dd, J = 15.1, 3.6 Hz, 1H), 2.44 - 2.56 (ovrlp, 3H), 2.29 $2.39(\mathrm{~m}, 1 \mathrm{H}), 2.12(\mathrm{td}, J=12.5,9.7 \mathrm{~Hz}, 1 \mathrm{H}), 1.63(\mathrm{ddd}, J=13.0,11.9,6.1 \mathrm{~Hz}, 1 \mathrm{H}), 1.56(\mathrm{dd}, J=15.1,2.4$ $\mathrm{Hz}, 1 \mathrm{H}), 1.00(\mathrm{~s}, 3 \mathrm{H}), 0.89$ (t, $J=7.1 \mathrm{~Hz}, 3 \mathrm{H}) .{ }^{13} \mathrm{C}$ NMR $\left(175 \mathrm{MHz}, \mathrm{CDCl}_{3}\right): \delta 216.5,200.8,169.6,164.8$, 152.0, 144.2, 133.3, 129.8, 129.4, 128.3, 126.8, 125.3, 78.5, 66.8, 62.03, 52.1, 46.8, 46.4, 45.7, 34.8, 33.5, 32.1, 31.8, 30.3, 20.6, 13.6. HRMS (ESI): Calucalted for $\mathrm{C}_{28} \mathrm{H}_{30} \mathrm{ClO}_{7}^{+}[\mathrm{M}+\mathrm{H}]^{+} 513.1675$, found 513.1680. IR (film, cm ${ }^{-1}$ ): 3507(br), 2911, 1724, 1697, 1658, 1625, 1451, 1350, 1277, 1177, 1074, 1027, 900 , 717. $[\alpha]_{D}{ }^{25}=-176\left(\mathrm{c}=1.1, \mathrm{CHCl}_{3}\right)$.
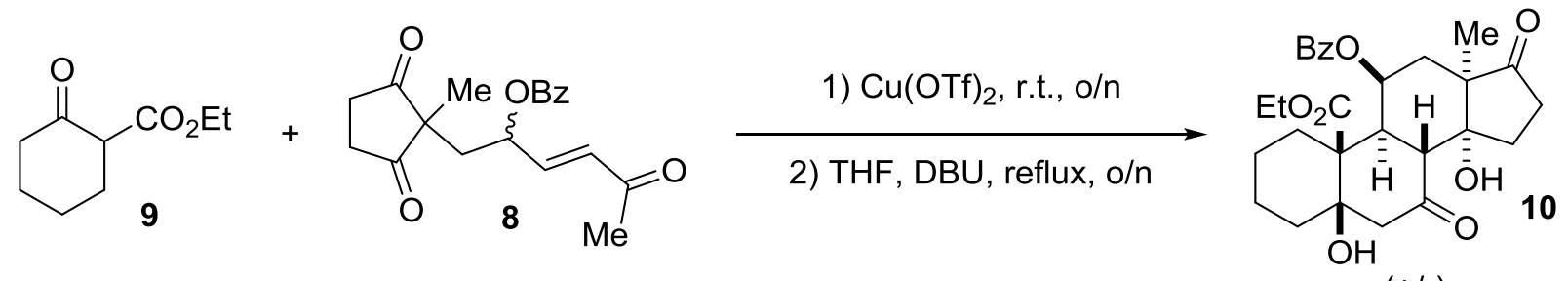

$(+/-)$

Compound 10. Racemic enone $8(328.0 \mathrm{mg}, 1.0 \mathrm{mmol})$, and ketoester 9 (500 mg, $2.94 \mathrm{mmol}$ ) were 
successively added to a flask with a stir bar and the vessel was purged with nitrogen. Then, $\mathrm{Cu}(\mathrm{OTf})_{2}(54$ $\mathrm{mg}, 0.015 \mathrm{mmol}$ ) was added and the reaction was allowed to stir at room temperature overnight. The next day, the residue was filtered through a plug of silica with diethyl ether as eluent. The crude mixture was then purified by flash column chromatography with $10 \% \rightarrow 50 \%$ EtOAc in hexanes. The resultant adduct was used for cyclization without further purification. It was solvated in THF ( $2.5 \mathrm{~mL})$, and 1,8diazabycyclo[5.4.0]undec-7-ene $(50.4 \mathrm{mg}, 49 \mu \mathrm{L}, 0.33 \mathrm{mmol})$ was added via syringe. The solution was allowed to reflux for $18 \mathrm{~h}$. Afterwards, the solution was cooled and concentrated in vacuo. The resulting residue was directly purified by flash column chromatography with $30 \%$ EtOAc in hexanes to afford title compound 10 (219.1 mg, 0.44) in 44\% unoptimized yield, $\mathrm{R}_{\mathrm{f}}=0.2$ (30\% EtOAc in hexanes).. The product was then crystallized from hexanes $/ \mathrm{CH}_{2} \mathrm{Cl}_{2}$ and submitted for $\mathrm{X}$-ray analysis. ${ }^{1} \mathbf{H} \mathbf{N M R}\left(500 \mathrm{MHz}, \mathrm{CDCl}_{3}\right): \delta$ $7.86(\mathrm{~d}, J=7.2 \mathrm{~Hz}, 2 \mathrm{H}), 7.54(\mathrm{t}, J=7.4 \mathrm{~Hz}, 1 \mathrm{H}), 7.42(\mathrm{t}, J=7.7 \mathrm{~Hz}, 2 \mathrm{H}), 5.41(\mathrm{~d}, J=1.1 \mathrm{~Hz}, 1 \mathrm{H}), 4.41(\mathrm{~s}, 1 \mathrm{H})$, $3.85-3.73(\mathrm{~m}, 2 \mathrm{H}), 3.71(\mathrm{~d}, J=12.6 \mathrm{~Hz}, 1 \mathrm{H}), 3.31(\mathrm{~s}, 1 \mathrm{H}), 2.58(\mathrm{dd}, J=15.1,3.3 \mathrm{~Hz}, 1 \mathrm{H}), 2.55-2.38(\mathrm{~m}$, ovrlp, $5 \mathrm{H}), 2.34-2.21(\mathrm{~m}, 1 \mathrm{H}), 2.08-2.21(\mathrm{~m}, 2 \mathrm{H}), 1.98(\mathrm{~d}, J=14.5 \mathrm{~Hz}, 1 \mathrm{H}), 1.63-1.45(\mathrm{~m}$, ovrlp, $6 \mathrm{H})$, $1.41-1.28(\mathrm{~m}, 1 \mathrm{H}), 0.98(\mathrm{~s}, 3 \mathrm{H}), 0.92(\mathrm{t}, J=7.1 \mathrm{~Hz}, 3 \mathrm{H}) .{ }^{13} \mathrm{C}$ NMR $\left(125 \mathrm{MHz}, \mathrm{CDCl}_{3}\right): \delta 216.11,212.81$, 174.1, 165.0, 133.2, 129.7, 129.6, 128.3, 77.5, 74.9, 67.4, 61.0, 54.1, 51.8, 51.6, 49.0, 40.2, 35.8, 34.8, 33.4, 31.7, 21.1, 20.4, 20.1, 13.5. IR (film, $\mathrm{cm}^{-1}$ ): 3504 (br), 2932, 1718 (ovrlp), 1451, 1270, 1177, 1071, 1026, 708.
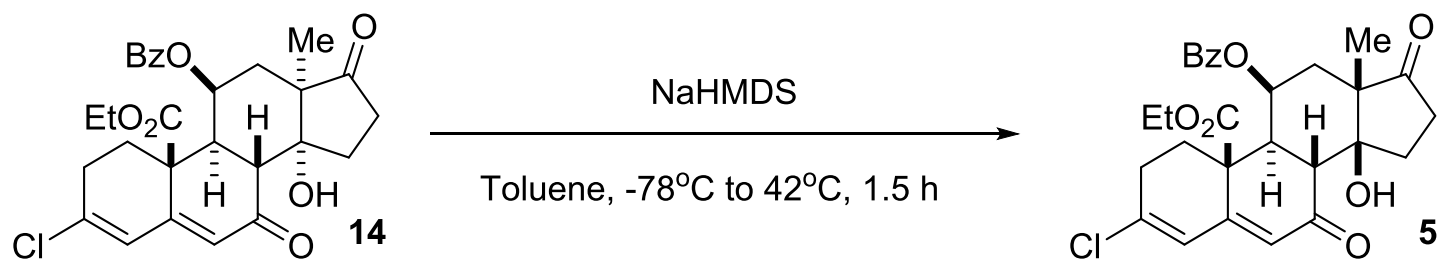

Compound 5. Steroid $14(2.0 \mathrm{~g}, 3.9 \mathrm{mmol})$ was solvated in dry Toluene $(30 \mathrm{~mL})$ and cooled to $-78^{\circ} \mathrm{C}$ under $\mathrm{N}_{2}$. Then, A solution of sodium bis(trimethylsilyl)amide $(860 \mathrm{mg}, 4.7 \mathrm{mmol})$ in toluene $(10 \mathrm{~mL})$ was added dropwise to the reaction vessel. The solution was allowed to stir at $-78^{\circ} \mathrm{C}$ for 1 hour before it was slowly warmed to room temperature and subsequently placed in a $42^{\circ} \mathrm{C}$ oil bath where it was stirred for an additional 45 minutes. The reaction was cooled and quenched with a saturated $\mathrm{NH}_{4} \mathrm{Cl}$ solution $(5 \mathrm{~mL})$, concentrated in vacuo, and directly adsorbed onto silica gel and purified by flash column chromatography with 25\% EtOAc in hexanes to afford title compound 5 (960 mg, $1.9 \mathrm{mmol}$ ) as an off white solid in $48 \%$ yield and 68\% BRSM, $\mathrm{R}_{\mathrm{f}}=0.5$ (50\% EtOAc in Hexanes). ${ }^{1} \mathbf{H}$ NMR $\left(700 \mathrm{MHz}, \mathrm{CDCl}_{3}\right): \delta$ $8.01(\mathrm{~d}, J=7.2 \mathrm{~Hz}, 2 \mathrm{H}), 7.61(\mathrm{t}, J=7.4 \mathrm{~Hz}, 1 \mathrm{H}), 7.47(\mathrm{t}, J=7.8 \mathrm{~Hz}, 2 \mathrm{H}), 6.35(\mathrm{~d}, J=2.1 \mathrm{~Hz}, 1 \mathrm{H}), 5.96(\mathrm{~s}, 1 \mathrm{H})$, $5.74(\mathrm{~d}, J=2.5 \mathrm{~Hz}, 1 \mathrm{H}), 5.24(\mathrm{~s}, 1 \mathrm{H}), 3.79(\mathrm{dq}, J=10.9,7.1 \mathrm{~Hz}, 1 \mathrm{H}), 3.66(\mathrm{~d}, J=13.6 \mathrm{~Hz}, 1 \mathrm{H}), 3.46(\mathrm{dq}, J=$ 10.9, 7.1 Hz, 1H), $2.96-2.83(\mathrm{~m}, 1 \mathrm{H}), 2.79(\mathrm{dd}, J=13.1,5.3 \mathrm{~Hz}, 1 \mathrm{H}), 2.64-2.56(\mathrm{~m}, 1 \mathrm{H}), 2.53(\mathrm{dd}, J=19.6$, $5.7 \mathrm{~Hz}, 1 \mathrm{H}$ ), 2.41 (ddd, $J=19.3,6.5,4.0 \mathrm{~Hz}, 1 \mathrm{H}), 2.28$ (dd, J=13.6, $2.2 \mathrm{~Hz}, 1 \mathrm{H}), 2.06-2.00(\mathrm{~m}, 2 \mathrm{H}), 1.90$ (dd, $J=15.6,3.1 \mathrm{~Hz}, 1 \mathrm{H}$ ), 1.65 (ddd, $J=13.3,11.3,6.1 \mathrm{~Hz}, 1 \mathrm{H}$ ), 1.45 (dd, $J=15.6,2.8 \mathrm{~Hz}, 1 \mathrm{H}), 1.21(\mathrm{~s}, 3 \mathrm{H}$ ), $0.93(\mathrm{t}, J=7.1 \mathrm{~Hz}, 3 \mathrm{H}) .{ }^{13} \mathrm{C}$ NMR $\left(175 \mathrm{MHz}, \mathrm{CDCl}_{3}\right): \delta 218.9,202.7,168.9,164.8,153.8,145.3,133.7$, 129.8, 129.2, 128.6, 126.0, 125.1, 80.9, 66.1, 62.2, 51.7, 49.1, 45.5, 45.2, 34.9, 33.1, 31.6, 30.0, 27.8, 16.9, 13.6. HRMS (ESI): Calculated for $\mathrm{C}_{28} \mathrm{H}_{30} \mathrm{ClO}_{7}^{+}[\mathrm{M}+\mathrm{H}]^{+}$513.1675, found 513.1680. IR (film, cm $\left.{ }^{-1}\right): 3485$

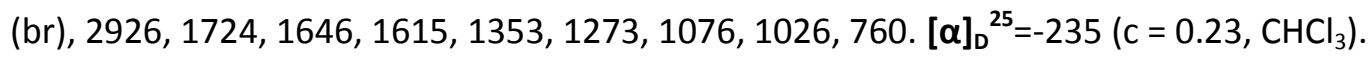




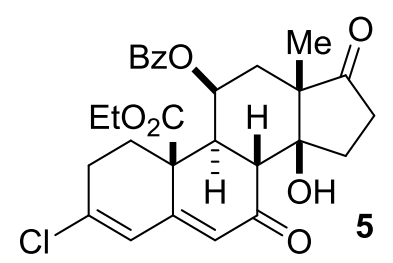

1) DIBALH, $-78^{\circ} \mathrm{C}$ to $60^{\circ} \mathrm{C}$, THF

2) $\mathrm{HCOOH}, \mathrm{H}_{2} \mathrm{O}, 85^{\circ} \mathrm{C}$

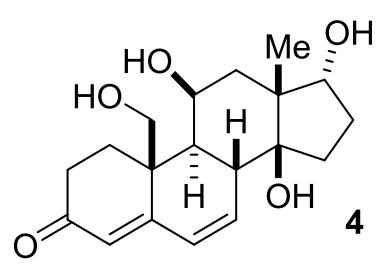

Compound 4. To a flame dried flask containing steroid $5(600.0 \mathrm{mg}, 1.17 \mathrm{mmol}$ ) in THF ( $35 \mathrm{~mL})$ under $\mathrm{N}_{2}$ and cooled to $-78^{\circ} \mathrm{C}$ was added diisobutyl aluminum hydride $(3.3 \mathrm{~g}, 4.2 \mathrm{~mL}, 23.4 \mathrm{mmol})$ dropwise. The reaction was allowed to stir at $-78^{\circ} \mathrm{C}$ for $1 \mathrm{~h}$ before being heated to $60^{\circ} \mathrm{C}$ for $12 \mathrm{~h}$. After completion, the reaction flask was cooled to $0^{\circ} \mathrm{C}$ and quenched catiously by the addition of water $(\sim 10 \mathrm{~mL})$ until the formation of a viscous emulsion. The solution was gradually acidifed with formic acid ( $8 \mathrm{~mL}$ total) until the emulsions dissipated and the solution became homogeneous $(\mathrm{pH}<4)$. The mixture was then heated to $85^{\circ} \mathrm{C}$ for $1 \mathrm{~h}$ and then quenched by the addition of a saturated $\mathrm{NaHCO}_{3}$ solution $(10 \mathrm{~mL})$ and solid $\mathrm{Na}_{2} \mathrm{CO}_{3}(2 \mathrm{~g})$. The suspension was then filtered through celite with excess $\mathrm{MeOH}$ and concentrated. The crude mixture was then adsorbed onto silica gel (with $\mathrm{MeOH}$ ) and purified directly by flash column chromatography with $5 \% \rightarrow 10 \% \mathrm{MeOH}$ in $\mathrm{CH}_{2} \mathrm{Cl}_{2}$ to afford title compound 4 (281 $\mathrm{mg}, 0.842 \mathrm{mmol}$ ) in 72\% yield and 8:1 d.r., $\mathrm{R}_{\mathrm{f}}=0.2\left(11 \% \mathrm{MeOH}\right.$ in $\mathrm{CH}_{2} \mathrm{Cl}_{2}$ ). ${ }^{1} \mathrm{H}$ NMR (700 MHz, $\mathrm{D}_{3} \mathrm{COD}$ ): $\delta 6.49$ (dd, $J=9.9,1.8$ $\mathrm{Hz}, 1 \mathrm{H}), 6.25(\mathrm{dd}, J=9.9,2.6 \mathrm{~Hz}, 1 \mathrm{H}), 5.69(\mathrm{~s}, 1 \mathrm{H}), 4.18(\mathrm{~d}, J=1.6 \mathrm{~Hz}, 1 \mathrm{H}), 4.04(\mathrm{t}, J=8.9 \mathrm{~Hz}, 1 \mathrm{H}), 3.75(\mathrm{~d}$, $J=12.2 \mathrm{~Hz}, 1 \mathrm{H}$ ), $3.43(\mathrm{~d}, J=12.2 \mathrm{~Hz}, 1 \mathrm{H}), 2.93(\mathrm{~d}, J=11.4 \mathrm{~Hz}, 1 \mathrm{H}$ ), $2.65-2.51$ (ovrlp, $2 \mathrm{H}$ ), 2.37 (dd, $J=$ 16.8, $4.8 \mathrm{~Hz}, 1 \mathrm{H}$ ), 2.01 (ddd, $J=13.5,9.3,4.2 \mathrm{~Hz}, 1 \mathrm{H}$ ), 1.84 (ddd, $J=14.6,12.8,4.8 \mathrm{~Hz}, 1 \mathrm{H}$ ), $1.76-1.64$ $(\mathrm{m}, 2 \mathrm{H}), 1.61(\mathrm{dd}, J=11.5,1.2 \mathrm{~Hz}, 1 \mathrm{H}), 1.52-1.42(\mathrm{~m}, 1 \mathrm{H}), 1.41-1.32$ (ovrlp, $2 \mathrm{H}$ ), $1.30(\mathrm{~s}, 3 \mathrm{H}) .{ }^{13} \mathrm{C}$ NMR (175 MHz, $\left.\mathrm{D}_{3} \mathrm{COD}\right): \delta$ 202.0, 162.9, 141.6, 129.7, 124.6, 84.1, 82.9, 64.9, 61.8, 51.1, 48.4, 43.3, 39.6, 36.4, 34.4, 30.3, 29.0, 28.9, 21.6. HRMS (ESI): Calculated for $\mathrm{C}_{19} \mathrm{H}_{27} \mathrm{O}_{5}{ }^{+}[\mathrm{M}+\mathrm{H}]^{+} 335.1853$, found 335.1855. IR

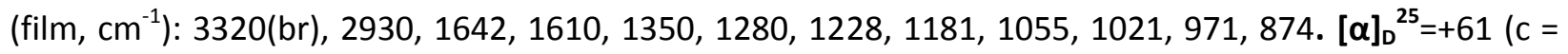
$1.0, \mathrm{MeOH})$.
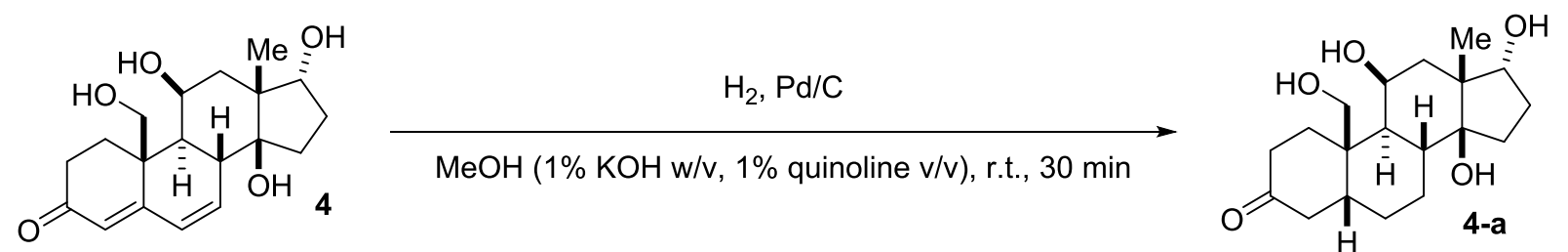

Compound 4-a. Tetra-ol 4 (60 mg, $0.180 \mathrm{mmol}$ ) and 10\% Pd on activated carbon (15 mg, 25\% w/w loading) was solvated in $5.2 \mathrm{~mL}$ of $\mathrm{MeOH}$ containing $1 \% \mathrm{KOH}(\mathrm{w} / \mathrm{v})$ and $1 \%$ quinoline $(\mathrm{v} / \mathrm{v})$. The reaction vessel was purged with $\mathrm{N}_{2}$, and then $\mathrm{H}_{2}$, and allowed to stir vigorously at room temperature under an atmosphere of $\mathrm{H}_{2}$. After 30 minutes, TLC showed complete conversion. The solution was quenched with $600 \mu \mathrm{L}$ of $\mathrm{pH}=7$ phosphate buffer. The crude mixture was directly adsorbed onto silica gel in vacuo and purified by flash column chromatography with $0 \% \rightarrow 5 \% \rightarrow 10 \% \mathrm{MeOH}$ in DCM to afford compound 4-a (51 mg, $0.151 \mathrm{mmol}$ ) exclusively in $84 \%$ yield as a white solid, $\mathrm{R}_{\mathrm{f}}=0.2\left(10 \% \mathrm{MeOH}\right.$ in $\left.\mathrm{CH}_{2} \mathrm{Cl}_{2}\right) .{ }^{1} \mathbf{H} \mathbf{~ N M R}$ (400 MHz, $\left.\mathrm{D}_{3} \mathrm{COD}\right): \delta 4.29(\mathrm{~d}, J=11.6 \mathrm{~Hz}, 1 \mathrm{H}), 4.21(\mathrm{~d}, J=2.3 \mathrm{~Hz}, 1 \mathrm{H}), 4.09(\mathrm{t}, J=8.8 \mathrm{~Hz}, 1 \mathrm{H}), 3.23(\mathrm{~d}, J=$ $11.6 \mathrm{~Hz}, 1 \mathrm{H}), 2.79(, J=15.4 \mathrm{~Hz}, 1 \mathrm{H}$, ) , $2.48(\mathrm{td}, J=14.7,5.7 \mathrm{~Hz}, 1 \mathrm{H}), 2.41-2.31(\mathrm{~m}, 1 \mathrm{H}), 2.29-2.13(\mathrm{~m}$, ovrlp, 3H), 2.13 - 2.01 (m, ovrlp, 2H), 2.00 - 1.84 (m, ovrlp, 3H), 1.84 - 1.68 (m, ovrlp, 2H), 1.63 - 1.32 (m, ovrlp, 5H), 1.32 - 1.22 (ovrlp, 4H). ${ }^{13} \mathrm{C}$ NMR (100 MHz, D $\mathrm{COD}$ ): $\delta$ 215.5, 86.0, 83.4, 68.5, 66.1, 47.8, 44.1, 43.0, 40.6, 40.5, 37.6, 36.6, 36.2, 32.2, 30.4, 28.7, 26.2, 21.1, 20.3. HRMS (ESI): Calculated for $\mathrm{C}_{19} \mathrm{H}_{31} \mathrm{O}_{5}{ }^{+}$ $[\mathrm{M}+\mathrm{H}]^{+}$339.2166, found 339.2158. IR (film, $\left.\mathrm{cm}^{-1}\right)$ : 3392 (br), 2940, 1536, 1512, 1466, 1251, 1177, 1033, 814. $[\alpha]_{D}^{25}=+5.7(c=0.7, \mathrm{MeOH})$. 

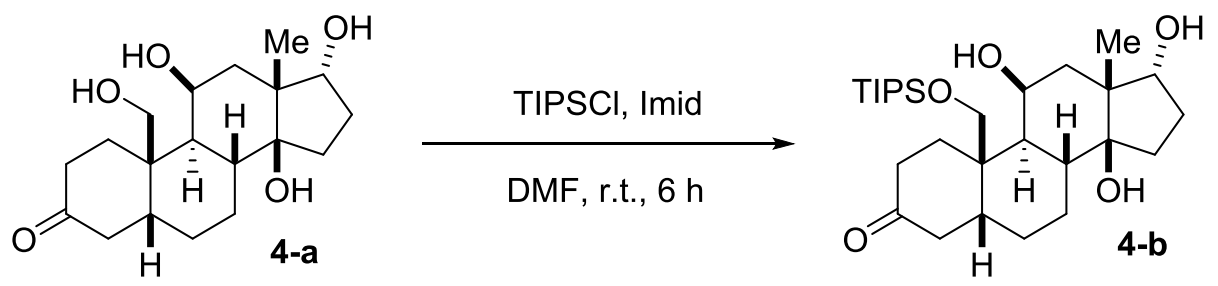

Compound 4-b. Steroid 4-a (40 mg, $0.118 \mathrm{mmol}$ ) and Imidazole (130 mg, $1.912 \mathrm{mmol}$ ) were solvated in dry DMF ( $5 \mathrm{~mL}$ ) at room temperature. Triisopropylsilyl chloride ( $155 \mu \mathrm{L}, 140 \mathrm{mg}, 0.724 \mathrm{mmol}$ ) was added by syringe. The reaction mixture was allowed to stir at ambient temperature for $6 \mathrm{~h}$, until all starting material was consumed. The solution was then quenched with a saturated $\mathrm{NaHCO}_{3}$ solution $(2 \mathrm{~mL})$ and diluted with EtOAc $(5 \mathrm{~mL})$. The organic layer was washed $3 x$ with 1:1 $\mathrm{H}_{2} \mathrm{O}$ :brine, dried over $\mathrm{Na}_{2} \mathrm{SO}_{4}$, filtered, concentrated, and directly purified by flash column chromatography with $0 \% \rightarrow 30 \% \rightarrow 50 \% \rightarrow$ $65 \%$ EtOAc in hexanes to afford title compound 4-b $(48 \mathrm{mg}, 0.097 \mathrm{mmol})$ in $82 \%$ yield as a white solid, $R_{\mathrm{f}}$ $=0.7\left(10 \% \mathrm{MeOH}\right.$ in $\left.\mathrm{CH}_{2} \mathrm{Cl}_{2}\right) .{ }^{1} \mathrm{H}$ NMR $\left(400 \mathrm{MHz}, \mathrm{CDCl}_{3}\right): \delta 4.70(\mathrm{~s}, 1 \mathrm{H}), 4.44(\mathrm{~d}, J=10.4 \mathrm{~Hz}, 1 \mathrm{H}), 4.24-$ $4.13(\mathrm{~m}$, ovrlp, $2 \mathrm{H}), 3.35(\mathrm{~d}, J=10.4 \mathrm{~Hz}, 1 \mathrm{H}), 2.64(\mathrm{t}, J=14.4 \mathrm{~Hz}, 1 \mathrm{H}), 2.48-2.39(\mathrm{~m}, 1 \mathrm{H}), 2.34-2.26(\mathrm{~m}$, ovrlp, 2H), 2.26 - 2.08 (m, ovrlp, 2H), 2.09 - 1.98 (m, ovrlp, 2H), 1.98 - 1.80 (m, ovrlp, 4H), 1.68 - 1.45 (m, ovrlp, 4H), 1.41 - 1.23 (m, ovrlp, 6H), $1.19-1.00$ (m, ovrlp, 21H). $\left.{ }^{13} \mathrm{C} \mathrm{NMR} \mathrm{(100} \mathrm{MHz,} \mathrm{CDCl}_{3}\right): \delta 211.8$, 85.0, 82.6, 69.4, 64.3, 46.7, 43.5, 42.0, 40.2, 39.3, 36.8, 35.7, 34.8, 30.9, 30.0, 28.0, 25.0, 19.8, 18.8, 18.0, 17.9, 11.8. HRMS (ESI): Calculated for $\mathrm{C}_{28} \mathrm{H}_{51} \mathrm{O}_{5} \mathrm{Si}^{+}[\mathrm{M}+\mathrm{H}]^{+}$495.3500, found 495.3509. IR (film, $\left.\mathrm{cm}^{-1}\right): 3408$ (br), 2941, 2866, 1709, 1462, 1264, 1058, 1016, 882, 734. [ $\alpha]_{\mathrm{D}}{ }^{25}=+1.8\left(\mathrm{c}=0.7, \mathrm{CHCl}_{3}\right)$
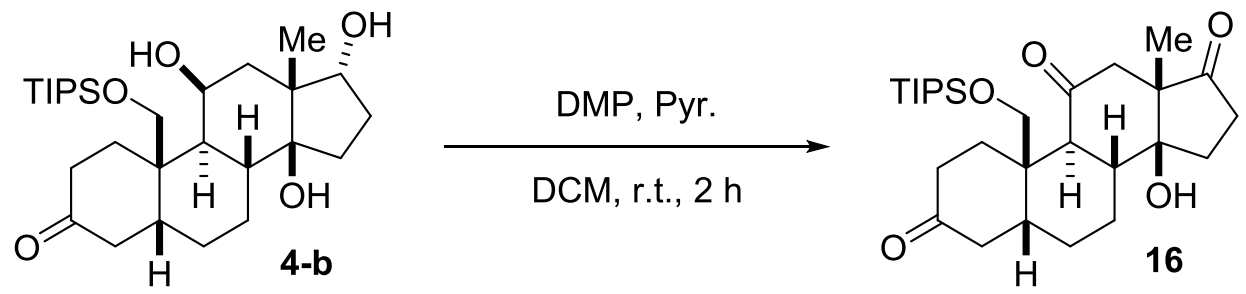

Compound 16. Compound 4-b (90 mg, $0.182 \mathrm{mmol}$ ) and the Dess-Martin periodinane (400 $\mathrm{mg}, 0.943$ mmol) was solvated in dry $\mathrm{CH}_{2} \mathrm{Cl}_{2}(4.0 \mathrm{~mL})$ and pyridine $(75 \mu \mathrm{L})$. The reaction mixture was allowed to stir at room temperature for $2 \mathrm{~h}$ until the starting material was consumed. The reaction was quenched with a saturated $\mathrm{Na}_{2} \mathrm{~S}_{2} \mathrm{O}_{3}$ solution $(2 \mathrm{~mL})$ and a saturated $\mathrm{NaHCO}_{3}$ solution $(2 \mathrm{~mL}$ ) and allowed to stir vigorously for 1 hour. The resulting mixture was extracted $3 x$ with $\mathrm{CH}_{2} \mathrm{Cl}_{2}$ (4 mL each), dried over $\mathrm{Na}_{2} \mathrm{SO}_{4}$, filtered, adsorbed onto silica gel, and directly purified by flash column chromatography with $30 \% \rightarrow 40 \%$ $\rightarrow 50 \%$ EtOAc in hexanes to afford title compound $16(80 \mathrm{mg}, 0.163 \mathrm{mmol})$ in $89 \%$ yield as a white solid, $\mathrm{R}_{\mathrm{f}}=0.3$ (50\% EtOAc in hexanes). ${ }^{1} \mathrm{H}$ NMR $\left(400 \mathrm{MHz}, \mathrm{CDCl}_{3}\right): \delta 4.28(\mathrm{~d}, J=9.5 \mathrm{~Hz}, 1 \mathrm{H}), 3.95(\mathrm{~d}, J=9.6 \mathrm{~Hz}$, $1 \mathrm{H}), 2.79(\mathrm{~d}, J=12.7 \mathrm{~Hz}, 1 \mathrm{H}), 2.66-2.30(\mathrm{~m}$, ovrlp, $7 \mathrm{H}), 2.26-2.05(\mathrm{~m}$, ovrlp, $6 \mathrm{H}), 2.00(\mathrm{~d}, J=13.3 \mathrm{~Hz}$, 1H), $1.97-1.87$ (m, ovrlp, 2H), $1.68-1.54(\mathrm{~m}, 1 \mathrm{H}), 1.51-1.40(\mathrm{~m}, 1 \mathrm{H}), 1.18-0.99$ (m, ovrlp, $24 \mathrm{H}) .{ }^{13} \mathrm{C}$ NMR $\left(100 \mathrm{MHz}, \mathrm{CDCl}_{3}\right): \delta 216.1,212.3,205.9,80.4,63.4,56.5,48.9,47.1,43.6,41.6,39.7,37.4,34.5$, 32.9, 30.8, 27.5, 25.5, 20.2, 18.1, 18.1, 13.4, 12.0. HRMS (ESI): Calculate for $\mathrm{C}_{28} \mathrm{H}_{46} \mathrm{O}_{5} \mathrm{SiNa}^{+}[\mathrm{M}+\mathrm{Na}]^{+}$ 513.3007, found 513.2996. IR (film, $\mathrm{cm}^{-1}$ ): 3457 (br), 2939, 2866, 1734, 1708, 1462,1210, 1094, 882. $[\alpha]_{D}^{25}=+68.7\left(\mathrm{c}=0.45, \mathrm{CHCl}_{3}\right)$. 

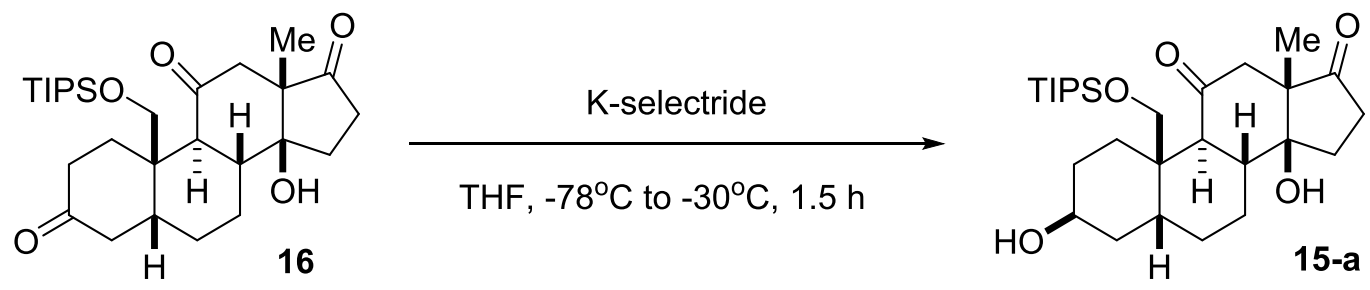

Compound 15-a. Triketone 16 (39 mg, $0.080 \mathrm{mmol}$ ) was solvated in dry THF (5 mL) and cooled to $-78^{\circ} \mathrm{C}$ under a $\mathrm{N}_{2}$ atmosphere. Then, $1 \mathrm{M} \mathrm{K}$-Selectride $(0.170 \mathrm{~mL}, 0.170 \mathrm{mmol})$ was added slowly via syringe. The reaction was allowed to warm to $-30^{\circ} \mathrm{C}$ where it was stirred for $1.5 \mathrm{~h}$ until the reaction was complete. The reaction was then quenched with a saturated $\mathrm{NH}_{4} \mathrm{Cl}$ solution $(2 \mathrm{~mL})$ and allowed to warm to room temperature. The mixture was then extracted $3 x$ with $\mathrm{CH}_{2} \mathrm{Cl}_{2}(4 \mathrm{~mL})$, dried over $\mathrm{Na}_{2} \mathrm{SO}_{4}$, filtered, adsorbed onto silica gel, and directly purified by flash column chromatography with $30 \% \rightarrow 40 \% \rightarrow 50 \%$ EtOAc in hexanes to afford title compound 15-a (30 mg, $0.061 \mathrm{mmol})$ in $77 \%$ yield as a white solid, $R_{\mathrm{f}}=$ 0.3 (50\% EtOAc in hexanes). ${ }^{1} \mathrm{H}$ NMR $\left(700 \mathrm{MHz}, \mathrm{CDCl}_{3}\right): \delta 4.14(\mathrm{~d}, J=9.7 \mathrm{~Hz}, 1 \mathrm{H}), 4.07(\mathrm{~s}, 1 \mathrm{H}), 3.97(\mathrm{~d}, J=$ $9.7 \mathrm{~Hz}, 1 \mathrm{H}), 2.76(\mathrm{~d}, J=12.8 \mathrm{~Hz}, 1 \mathrm{H}), 2.57-2.53(\mathrm{~m}, 2 \mathrm{H}), 2.48-2.41(\mathrm{~m}, 2 \mathrm{H}), 2.35(\mathrm{~d}, J=13.0 \mathrm{~Hz}, 1 \mathrm{H})$, $2.26(\mathrm{td}, J=12.6,3.9 \mathrm{~Hz}, 1 \mathrm{H}), 2.09(\mathrm{ddd}, J=13.6,5.8,5.3 \mathrm{~Hz}, 1 \mathrm{H}), 2.00-1.91(\mathrm{~m}, 3 \mathrm{H}), 1.84-1.77(\mathrm{~m}$, ovrlp, 3H), 1.64 - $1.55(\mathrm{~m}, 2 \mathrm{H}), 1.51-1.43(\mathrm{~m}, 2 \mathrm{H}), 1.37-1.29(\mathrm{~m}, 2 \mathrm{H}), 1.25(\mathrm{dd}, J=8.9,3.5 \mathrm{~Hz}, 1 \mathrm{H}), 1.17$ - 1.05 (m, ovrlp, 21H), 1.02 (s, 3H). ${ }^{13} \mathrm{C}$ NMR $\left(175 \mathrm{MHz} \mathrm{CDCl}_{3}\right): \delta 216.7,206.9,80.8,66.1,64.6,56.6,47.4$, 46.5, 43.5, 40.4, 33.3, 33.0, 28.3, 28.0, 27.5, 25.6, 22.7, 20.2, 18.2, 18.1, 13.6, 12.0. HRMS (ESI): Calculated for $\mathrm{C}_{28} \mathrm{H}_{49} \mathrm{O}_{5} \mathrm{Si}^{+}[\mathrm{M}+\mathrm{H}]^{+}$493.3344, found 493.3341. IR (film, $\mathrm{cm}^{-1}$ ): 3473 (br), 2928, 2862, 1737, $1718,1460,1378,1097 .[\alpha]_{D}^{25}=+68.7\left(c=1.13, \mathrm{CHCl}_{3}\right)$.
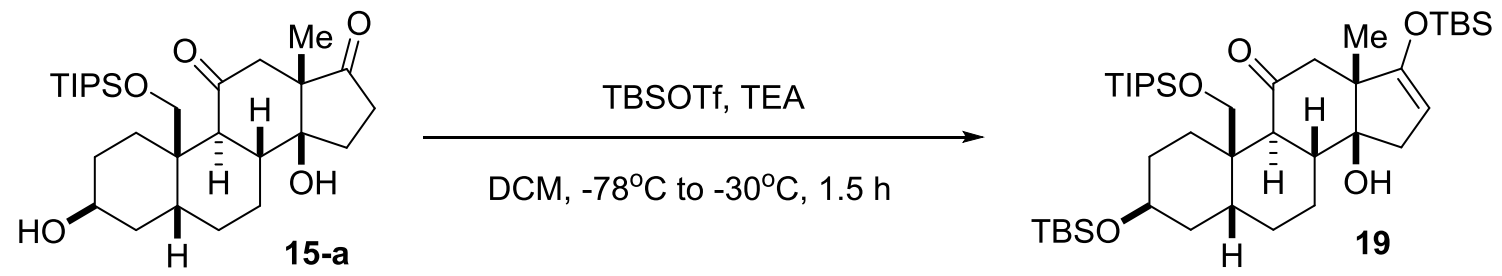

Compound 19. Diketone 15-a (29 mg, $0.059 \mathrm{mmol})$ was solvated in dry $\mathrm{CH}_{2} \mathrm{Cl}_{2}(2.5 \mathrm{~mL})$ and triethylamine $(225 \mu \mathrm{L})$ and cooled to $-78^{\circ} \mathrm{C}$ under a $\mathrm{N}_{2}$ atmosphere. Then, Tert-butyldimethylsilyl trifluoromethanesulfonate $(135 \mu \mathrm{L}, 110 \mathrm{mg}, 0.495 \mathrm{mmol})$ was added via syringe. The reaction was allowed to warm to $-30^{\circ} \mathrm{C}$ where it was stirred for $1.5 \mathrm{~h}$ until the reaction was complete. The solution was then quenched with a saturated $\mathrm{NaHCO}_{3}$ solution $(1 \mathrm{~mL})$ and extracted $3 x$ with $\mathrm{CH}_{2} \mathrm{Cl}_{2}(2 \mathrm{~mL})$, dried over $\mathrm{Na}_{2} \mathrm{SO}_{4}$, filtered, concentrated, and directly purified by flash column chromatography with $0 \% \rightarrow$ $5 \%$ EtOAc in hexanes to afford title compound $19(40 \mathrm{mg}, 0.056 \mathrm{mmol})$ in $95 \%$ yield as a white solid, $\mathrm{R}_{\mathrm{f}}=$ 0.7 (10\% EtOAc in hexanes). ${ }^{1} \mathrm{H}$ NMR $\left(700 \mathrm{MHz}, \mathrm{CDCl}_{3}\right): \delta 4.41(\mathrm{~s}, 1 \mathrm{H}), 4.11(\mathrm{~d}, J=9.8 \mathrm{~Hz}, 1 \mathrm{H}), 4.02(\mathrm{~s}$, $1 \mathrm{H}), 3.56(\mathrm{~d}, J=9.8 \mathrm{~Hz}, 1 \mathrm{H}), 2.69(\mathrm{dd}, J=16.5,1.8 \mathrm{~Hz}, 1 \mathrm{H}), 2.63(\mathrm{~d}, J=12.7 \mathrm{~Hz}, 1 \mathrm{H}), 2.44(\mathrm{td}, J=12.4,3.1$ $\mathrm{Hz}, 1 \mathrm{H}$ ), 2.27 (ovrlp, 2H), 2.08 (d, $J=12.8 \mathrm{~Hz}, 1 \mathrm{H}$ ), 2.03 (dd, $J=16.5,2.5 \mathrm{~Hz}, 1 \mathrm{H}$ ), 1.93 (d, $J=13.3 \mathrm{~Hz}, 1 \mathrm{H}$ ), $1.86(\mathrm{~m}$, ovrlp, $2 \mathrm{H}), 1.74-1.68(\mathrm{~m}$, ovrlp, $2 \mathrm{H}), 1.65(\mathrm{t}, J=13.7 \mathrm{~Hz}, 1 \mathrm{H}), 1.51(\mathrm{~m}, 1 \mathrm{H}), 1.36$ - 1.22 (m, ovrlp, 3H), 1.11 - $1.02(\mathrm{~m}, 24 \mathrm{H}), 0.92(\mathrm{~s}, 9 \mathrm{H}), 0.87(\mathrm{~s}, 9 \mathrm{H}), 0.16(\mathrm{~s}, 3 \mathrm{H}), 0.15(\mathrm{~s}, 3 \mathrm{H}), 0.010(\mathrm{~s}, 3 \mathrm{H}), 0.007(\mathrm{~s}, 3 \mathrm{H})$. ${ }^{13} \mathrm{C}$ NMR $\left(175 \mathrm{MHz}, \mathrm{CDCl}_{3}\right): \delta 212.0,156.0,95.7,80.2,68.4,66.8,53.6,49.3,46.6,43.7,41.7,38.0,34.9$, 30.6, 28.8, 26.8, 25.8, 25.64, 25.61, 24.8, 20.3, 19.3, 18.1, 18.1, 18.07, 18.05, 12.0, -4.7, -4.8, -4.9, -5.1. HRMS (ESI): Calculated for $\mathrm{C}_{40} \mathrm{H}_{77} \mathrm{O}_{5} \mathrm{Si}_{3}{ }^{+}[\mathrm{M}+\mathrm{H}]^{+} 721.5073$, found 721.5078 . 


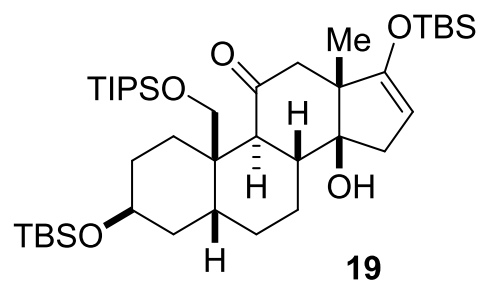

1) $\mathrm{Li}, \mathrm{NH}_{3},-78^{\circ} \mathrm{C}, 30 \mathrm{~min}$

2) TBAF, THF, $-78^{\circ} \mathrm{C}, 5 \mathrm{~min}$

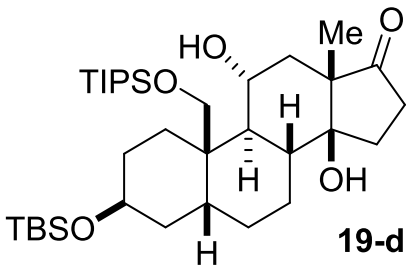

Compound 19-d. Gaseous ammonia $(\sim 1.5 \mathrm{~mL})$ was condensed at $-78^{\circ} \mathrm{C}$ under $\mathrm{N}_{2}$ and a small chip of lithium metal ( $2 \mathrm{mg}$ ) was added and allowed to dissolve. After 30 minutes, Compound 19 (9.1 mg, $0.0125 \mathrm{mmol})$ dissolved in dry THF $(0.5 \mathrm{~mL})$ was slowly added to the deep blue reaction ammonia solution via syringe and allowed to stir at $-78^{\circ} \mathrm{C}$ for 30 minutes. Then, the reaction was quenched dropwise by the addition of a saturated $\mathrm{NH}_{4} \mathrm{Cl}$ solution until the blue color had completely dissipated. The solution was allowed to warm to room temperature as the ammonia boiled off. The remaining mixture was extracted $2 x$ with $\mathrm{CH}_{2} \mathrm{Cl}_{2}(2 \mathrm{~mL})$, dried over $\mathrm{Na}_{2} \mathrm{SO}_{4}$, filtered, and concentrated. The crude mixture was then solvated in dry THF $(1 \mathrm{~mL})$ and cooled to $-78^{\circ} \mathrm{C}$ under $\mathrm{N}_{2}$. $1 \mathrm{M}$ Tetrabutylammonium fluoride in THF (15 $\mu \mathrm{L}, 0.015 \mathrm{mmol})$ was added via syringe and the reaction was stirred for precisely 5 minutes before being quenched with a saturated $\mathrm{NH}_{4} \mathrm{Cl}$ solution $(1 \mathrm{~mL})$ and warmed to room temperature. The crude mixture was then extracted $3 x$ with $\mathrm{CH}_{2} \mathrm{Cl}_{2}(2 \mathrm{~mL})$, dried over $\mathrm{Na}_{2} \mathrm{SO}_{4}$, filtered, adsorbed onto silica gel, and directly purified by flash column chromatography with $10 \% \rightarrow 20 \% \rightarrow 35 \%$ EtOAc in hexanes to afford title compound $19-\mathrm{d}(5.5 \mathrm{mg}, 0.0091 \mathrm{mmol})$ in $73 \%$ yield as a white solid, $\mathrm{R}_{\mathrm{f}}=$ 0.7 (50\% EtOAc in hexanes). ${ }^{1} \mathrm{H}$ NMR $\left(500 \mathrm{MHz}, \mathrm{CDCl}_{3}\right): \delta 4.27(\mathrm{~d}, J=9.8 \mathrm{~Hz}, 1 \mathrm{H}), 4.06(\mathrm{~s}, 1 \mathrm{H}), 3.96(\mathrm{t}, J=$ $10.6 \mathrm{~Hz}, 1 \mathrm{H}), 3.43(\mathrm{~d}, J=9.8 \mathrm{~Hz}, 1 \mathrm{H}), 3.15(\mathrm{~d}, J=2.8 \mathrm{~Hz}, 1 \mathrm{H}), 2.53-2.21(\mathrm{~m}$, ovrlp, 4H), 1.94 - $1.48(\mathrm{~m}$, ovrlp, 11H), 1.47 - 1.35 (m, ovrlp, 2H), $1.31-1.05$ (m, ovrlp, 26H), $0.88(\mathrm{~s}, 9 \mathrm{H}), 0.03(\mathrm{~s}, 3 \mathrm{H}), 0.02$ (s, 3H). ${ }^{13} \mathrm{C}$ NMR $\left(175 \mathrm{MHz}_{2} \mathrm{CDCl}_{3}\right): \delta$ 219.9, 82.3, 70.3, 69.7, 66.5, 53.8, 41.7, 41.5, 40.6, 40.3, 35.2, 34.4, 33.0, 29.7, 28.8, 27.6, 27.2, 26.7, 25.8, 18.8, 18.1, 18.0, 14.3, 11.9, -4.9. HRMS (ESI): Calculated for $\mathrm{C}_{34} \mathrm{H}_{65} \mathrm{O}_{5} \mathrm{Si}_{2}{ }^{+}$ $[\mathrm{M}+\mathrm{H}]^{+}$609.4371, found 609.4379. IR (film, cm ${ }^{-1}$ ): 3483 (br), 2928, 2865, 1731, 1460, 1252, 1065, 830. $[\alpha]_{D}^{25}=-1.7\left(c=0.57, \mathrm{CHCl}_{3}\right)$.

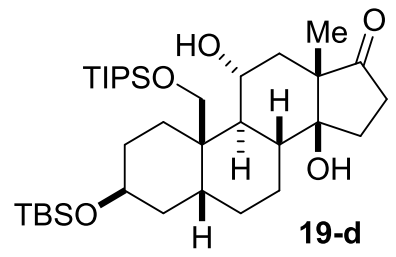

1) $\mathrm{H}_{2} \mathrm{NNH}_{2}-\mathrm{H}_{2} \mathrm{O}$, TEA, EtOH, $50^{\circ} \mathrm{C}, 6 \mathrm{~h}$

2) $I_{2}$, TEA, THF, r.t., $1 \mathrm{~h}$

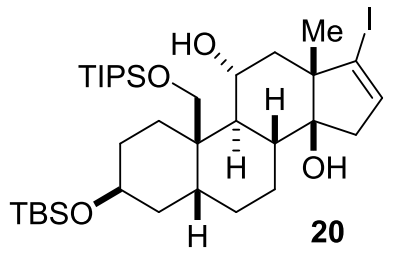

Compound 20. Compound 19-d $(5.5 \mathrm{mg}, 0.0091 \mathrm{mmol})$ was solvated in $\mathrm{EtOH}(1.0 \mathrm{~mL})$ and triethylamine $(25 \mu \mathrm{L})$. Hydrazine monohydrate $(9 \mathrm{mg}, 0.18 \mathrm{mmol})$ was then added and the solution was heated to $50^{\circ} \mathrm{C}$ and stirred for $6 \mathrm{~h}$ until all starting material was consumed. The resulting mixture was cooled to room temperature and concentrated in vacuo. The crude oil was then redissolved in THF $(1.0 \mathrm{~mL})$ and triethylamine $(25 \mu \mathrm{L})$. A solution of $\mathrm{I}_{2}(25 \mathrm{mg}, 0.0985 \mathrm{mmol})$ in THF $(1 \mathrm{~mL})$ was prepared and $0.25 \mathrm{~mL}$ of this solution was added to the reaction vessel. After 10 minutes, additional $\mathrm{I}_{2}$ solution $(0.30 \mathrm{~mL})$ was added until a brown color persisted. The mixture was stirred at room temperature for 1 hour until it was complete. The solution was then quenched with a saturated $\mathrm{Na}_{2} \mathrm{~S}_{2} \mathrm{O}_{3}$ solution $(1 \mathrm{~mL})$ and a saturated 
$\mathrm{NaHCO}_{3}$ solution $(1 \mathrm{~mL})$ and stirred vigorously for 20 minutes. The resulting mixture was then extracted $3 x$ with EtOAc ( $2 \mathrm{~mL}$ each), dried over $\mathrm{Na}_{2} \mathrm{SO}_{4}$, filtered, adsorbed onto silica gel, and directly purified by flash column chromatography with $0 \% \rightarrow 5 \% \rightarrow 10 \% \rightarrow 20 \%$ EtOAc in hexanes to afford title compound 20 (6.0 mg, $0.0084 \mathrm{mmol})$ in $90 \%$ yield, $\mathrm{R}_{\mathrm{f}}=0.9$ (50\% EtOAc in hexanes). ${ }^{1} \mathbf{H}$ NMR $\left(700 \mathrm{MHz}, \mathrm{CDCl}_{3}\right): \delta$ $6.13(\mathrm{~s}, 1 \mathrm{H}), 4.30(\mathrm{~d}, J=9.8 \mathrm{~Hz}, 1 \mathrm{H}), 4.04(\mathrm{~s}, 1 \mathrm{H}), 3.83(\mathrm{td}, J=12.4,3.3 \mathrm{~Hz}, 1 \mathrm{H}), 3.43(\mathrm{~d}, J=3.9 \mathrm{~Hz}, 1 \mathrm{H})$, $3.39(\mathrm{~d}, J=9.8 \mathrm{~Hz}, 1 \mathrm{H}), 2.66(\mathrm{dd}, J=16.5,1.0 \mathrm{~Hz}, 1 \mathrm{H}), 2.31-2.24(\mathrm{~m}$, ovrlp, $2 \mathrm{H}), 1.91$ (dd, $J=13.2,2.9 \mathrm{~Hz}$, 1H), 1.88 - 1.75 (m, ovrlp, 3H), 1.75 - 1.67 (m, ovrlp, 2H), 1.68 - 1.47 (m, ovrlp, 4H), 1.23 - 1.07 (m, ovrlp, 28H), 0.87 (s, 9H), $0.02(\mathrm{~s}, 3 \mathrm{H}), 0.01(\mathrm{~s}, 3 \mathrm{H}) .{ }^{13} \mathrm{C}$ NMR $\left(175 \mathrm{MHz}, \mathrm{CDCl}_{3}\right): \delta 133.7,110.2,82.1,70.5,69.1$, 66.6, 55.3, 46.1, 43.1, 41.8, 41.0, 40.5, 35.2, 34.5, 28.8, 27.1, 26.7, 25.8, 20.1, 19.6, 18.1, 18.0, 11.9, -4.9. HRMS (ESI): Calculated for $\mathrm{C}_{34} \mathrm{H}_{64} \mathrm{IO}_{4} \mathrm{Si}_{2}{ }^{+}[\mathrm{M}+\mathrm{H}]^{+} 719.3382$, found 719.3381. IR (film, $\mathrm{cm}^{-1}$ ): 3450 (br), $2926,2856,1462,1380,1253,1053,828 .[\alpha]_{D}{ }^{25}=-8.1\left(c=0.53, \mathrm{CHCl}_{3}\right)$.
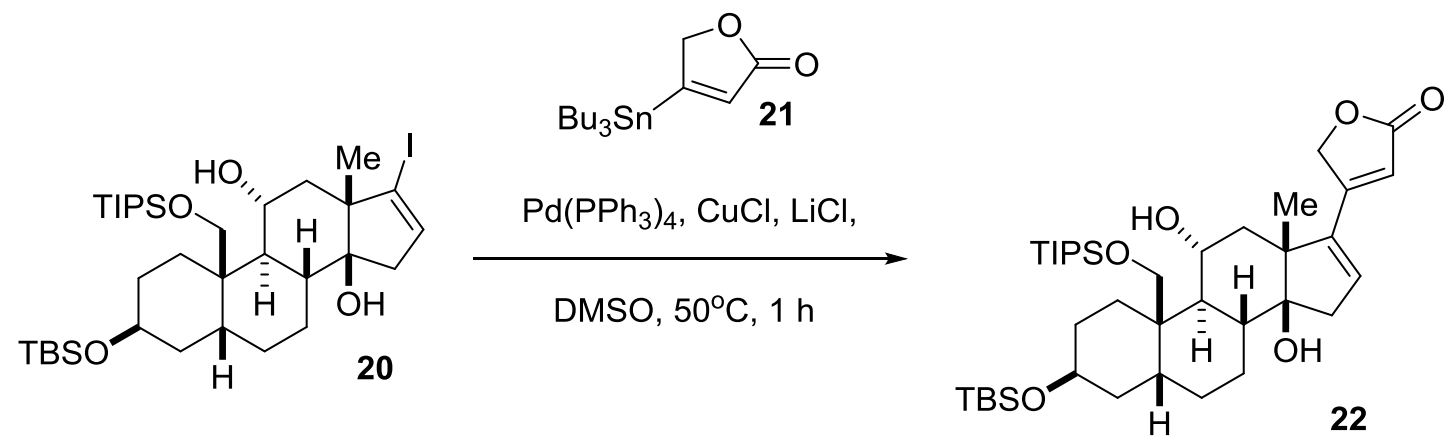

Compound 22. Vinyl lodide 20 (15.0 mg, $0.021 \mathrm{mmol})$ along with $\mathrm{Pd}\left(\mathrm{PPh}_{3}\right)_{4}(5 \mathrm{mg}, 0.0043 \mathrm{mmol}), \mathrm{CuCl}$ (31 mg, $0.313 \mathrm{mmol}$ ), and $\mathrm{LiCl}\left(18 \mathrm{mg}, 0.425 \mathrm{mmol}\right.$ ) was solvated in dry DMSO $(1.1 \mathrm{~mL})$ under a $\mathrm{N}_{2}$ atmosphere and stannyl butenolide $21(30 \mathrm{mg}, 0.0804 \mathrm{mmol}$ ) was added via syringe. The reaction solution was heated to $50^{\circ} \mathrm{C}$ for 1 hour until the starting material was consumed. The solution was then quenched with $\mathrm{pH}=7$ phosphate buffer $(1 \mathrm{~mL})$ and extracted $4 x$ with EtOAc $(1.5 \mathrm{~mL}$ each). The organic layers were combined, dried over $\mathrm{Na}_{2} \mathrm{SO}_{4}$, filtered, adsorbed onto silica gel, and directly purified by flash column chromatography with $0 \% \rightarrow 10 \% \rightarrow 20 \% \rightarrow 30 \%$ EtOAc in hexanes to afford title compound 22 (13.0 mg, $0.0193 \mathrm{mmol}$ ) in 92\% yield, $\mathrm{R}_{\mathrm{f}}=0.3$ (30\% EtOAc in hexanes). ${ }^{1} \mathrm{H} \mathbf{N M R}\left(700 \mathrm{MHz}, \mathrm{CDCl}_{3}\right): \delta 6.11$ $(\mathrm{s}, 1 \mathrm{H}), 6.00(\mathrm{~s}, 1 \mathrm{H}), 5.00(\mathrm{dd}, J=16.2,1.0 \mathrm{~Hz}, 1 \mathrm{H}), 4.92(\mathrm{dd}, J=16.2,1.0 \mathrm{~Hz}, 1 \mathrm{H}), 4.34(\mathrm{~d}, J=9.8 \mathrm{~Hz}, 1 \mathrm{H})$, $4.05(\mathrm{~s}, 1 \mathrm{H}), 3.93-3.85(\mathrm{~m}, 1 \mathrm{H}), 3.52(\mathrm{~d}, J=3.8 \mathrm{~Hz}, 1 \mathrm{H}), 3.40(\mathrm{~d}, J=9.8 \mathrm{~Hz}, 1 \mathrm{H}), 2.81(\mathrm{~d}, J=18.2 \mathrm{~Hz}, 1 \mathrm{H})$, $2.41(\mathrm{dd}, J=18.5,3.3 \mathrm{~Hz}, 1 \mathrm{H}), 2.30(\mathrm{~d}, J=13.9 \mathrm{~Hz}, 1 \mathrm{H}), 2.13(\mathrm{dd}, J=13.2,3.0 \mathrm{~Hz}, 1 \mathrm{H}), 1.91-1.66(\mathrm{~m}, 6 \mathrm{H})$, 1.66 - $1.48(\mathrm{~m}, 3 \mathrm{H}), 1.36-1.32$ (m, ovrlp, 4H), $1.31-1.15$ (m, ovrlp, 6H), $1.14-1.08(\mathrm{~m}, 18 \mathrm{H}), 0.088(\mathrm{~s}$, $9 \mathrm{H}), 0.025$ (s, 3H), $0.018(\mathrm{~s}, 3 \mathrm{H}) .{ }^{13} \mathrm{C}$ NMR $\left(175 \mathrm{MHz}, \mathrm{CDCl}_{3}\right): \delta 174.1,157.9,143.4,131.8,112.9,85.3$, 71.6, 70.6, 69.0, 66.5, 52.7, 47.1, 41.8, 40.9, 40.5, 40.5, 35.3, 34.6, 29.7, 28.8, 27.2, 26.7, 25.8, 20.2, 18.1, 18.0, 18.0, 12.0, -4.9. HRMS (ESI): Calculated for $\mathrm{C}_{38} \mathrm{H}_{67} \mathrm{O}_{6} \mathrm{Si}_{2}{ }^{+}[\mathrm{M}+\mathrm{H}]^{+} 675.4471$, found 675.4466. IR (film, $\left.\mathrm{cm}^{-1}\right): 3473(\mathrm{br}), 2925,2856,1747,1622,1462,1255,1045,828 .[\alpha]_{\mathrm{D}}^{25}=+19.5\left(\mathrm{c}=0.3, \mathrm{CHCl}_{3}\right)$. 

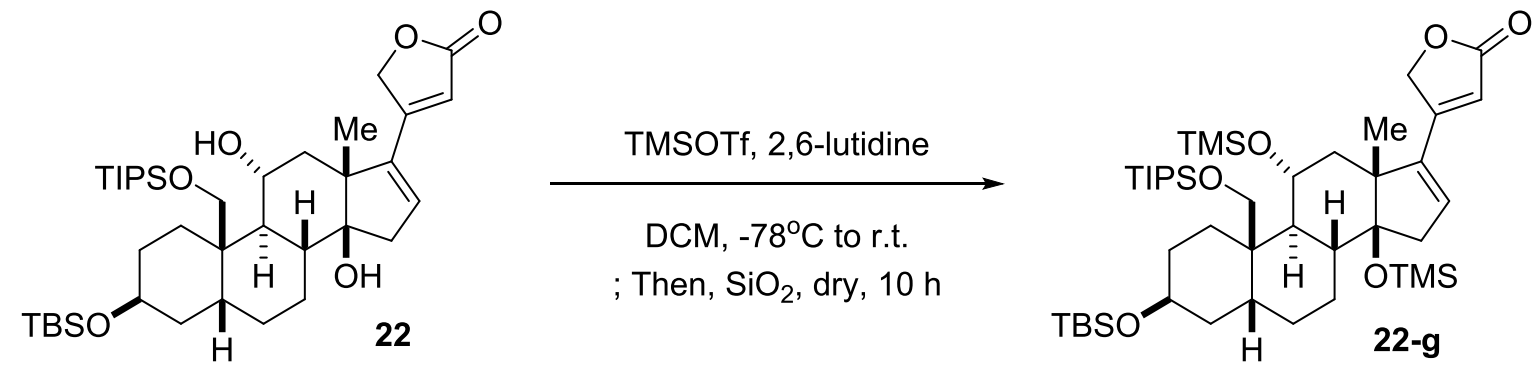

Compound 22-g. Compound 22 (13.0 mg, $0.0193 \mathrm{mmol}$ ) and 2,6 - lutidine ( $28 \mu \mathrm{L}, 25.9 \mathrm{mg}, 0.242 \mathrm{mmol})$ were solvated in dry $\mathrm{CH}_{2} \mathrm{Cl}_{2}(4 \mathrm{~mL})$ and cooled to $-78^{\circ} \mathrm{C}$ under a $\mathrm{N}_{2}$ atmosphere. Then, trimethylsilyl trifluoromethanesulfonate $(22 \mu \mathrm{L}, 27.0 \mathrm{mg}, 0.122 \mathrm{mmol}$ ) was added via syringe and the reaction was allowed to warm to room temperature. After $2 \mathrm{~h}$, the reaction was quenched with a saturated $\mathrm{NaHCO}_{3}$ solution (1.5 mL). The crude mixture was then extracted $3 x$ with $\mathrm{CH}_{2} \mathrm{Cl}_{2}\left(2 \mathrm{~mL}\right.$ each), dried over $\mathrm{Na}_{2} \mathrm{SO}_{4}$, filtered, and azeotroped $4 x$ with toluene $(2 \mathrm{~mL}$ each) to remove remaining 2,6-lutidine. The crude material was then solvated in $\mathrm{CH}_{2} \mathrm{Cl}_{2}(5 \mathrm{~mL})$ and adsorbed onto silica gel $(5 \mathrm{~g})$. The silica gel was allowed to sit at room temperature overnight. Then, the crude material was washed off the silica gel by filtering and eluting with $100 \%$ EtOAc. The filtrate was concentrated and directly purified by flash column chromatography with $0 \% \rightarrow 5 \%$ EtOAc in hexanes to afford title compound $\mathbf{2 2}-\mathrm{g}$ (10.0 $\mathrm{mg}, 0.0122 \mathrm{mmol})$ in 64\% yield, $\mathrm{R}_{\mathrm{f}}=0.4$ (10\% EtOAc in hexanes). ${ }^{1} \mathrm{H}$ NMR $\left(500 \mathrm{MHz}, \mathrm{CDCl}_{3}\right): \delta 6.06(\mathrm{t}, \mathrm{J}=2.5 \mathrm{~Hz}, 1 \mathrm{H}), 5.95$ (s, $1 \mathrm{H}), 5.01(\mathrm{dd}, J=16.2,1.5 \mathrm{~Hz}, 1 \mathrm{H}), 4.92(\mathrm{dd}, J=16.2,1.5 \mathrm{~Hz}, 1 \mathrm{H}), 4.02(\mathrm{~s}, 1 \mathrm{H}), 3.82-3.93(\mathrm{~m}$, ovrlp, 2H), $3.65(\mathrm{~d}, J=9.5 \mathrm{~Hz}, 1 \mathrm{H}), 2.87(\mathrm{~d}, J=17.9 \mathrm{~Hz}, 1 \mathrm{H}), 2.44(\mathrm{dd}, J=18.9,3.3 \mathrm{~Hz}, 1 \mathrm{H}), 2.25(\mathrm{~d}, J=11.9 \mathrm{~Hz}, 1 \mathrm{H})$, $1.93-1.70(\mathrm{~m}, 8 \mathrm{H}), 1.63-1.52(\mathrm{~m}, 1 \mathrm{H}), 1.44-1.35(\mathrm{~m}, 2 \mathrm{H}), 1.24-1.05(\mathrm{~m}, 27 \mathrm{H}), 0.88(\mathrm{~s}, 9 \mathrm{H}), 0.023(\mathrm{~s}$, $9 \mathrm{H}), 0.014(\mathrm{~s}, 6 \mathrm{H}), 0.007$ (s, 9H). ${ }^{13} \mathrm{C}$ NMR $\left(175 \mathrm{MHz}, \mathrm{CDCl}_{3}\right): \delta 174.5,158.4,143.3,133.3,111.8,88.7$, 71.6, 67.6, 67.4, 67.2, 52.8, 46.1, 42.7, 40.8, 40.3, 39.8, 34.9, 30.6, 29.3, 26.9, 25.9, 25.3, 21.6, 19.7, 18.3, $18.2,18.1,12.0,2.6,0.8,-4.9,-4.9$.

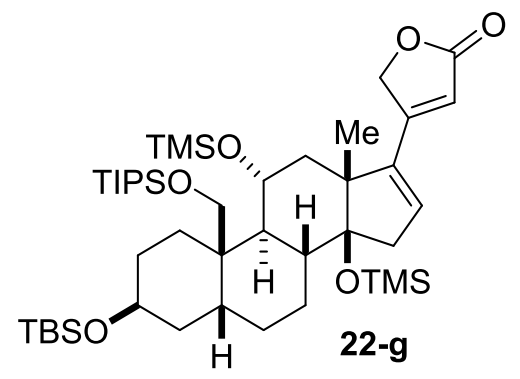

1) $\mathrm{H}_{2}, \mathrm{Pd} / \mathrm{C}, \mathrm{EtOAc}, 30 \mathrm{~min}$

2) $\mathrm{HF}, \mathrm{MeCN}, \mathrm{DCM}, \mathrm{H}_{2} \mathrm{O}$

r.t., 3 days

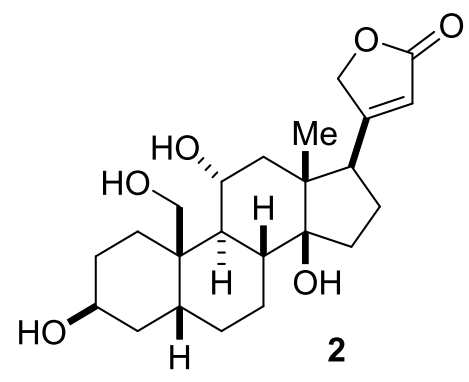

19-Hydroxysarmentogenin (2). Compound $22-\mathrm{g}(10.0 \mathrm{mg}, 0.0122 \mathrm{mmol})$ and $10 \%$ Palladium on activated carbon $\left(10 \mathrm{mg}\right.$ ) were solvated in EtOAc $(1.5 \mathrm{~mL})$ and the reaction vessel was purged with $\mathrm{N}_{2}$, and then with $\mathrm{H}_{2}$, and the solution was allowed to stir vigorously under an atmosphere of $\mathrm{H}_{2}$. After 30 minutes, all starting material was consumed. The reaction solution was then filtered through a pad of celite and silica gel with 100\% EtOAc. The resulting crude filtrate was a 2.5:1 diastereomeric mixture of C17 epimers. The minor diastereomer was then removed by flash column chromatography with $0 \% \rightarrow$ $5 \% \rightarrow 10 \%$ EtOAc in hexanes. The material was then immediately subjected to HF deprotection without further purification. The residue was solvated in $450 \mu \mathrm{L}$ of $1: 1 \mathrm{CH}_{2} \mathrm{Cl}_{2}: \mathrm{MeCN}$. Then, a stock solution of 18:3:2 MeCN: $\mathrm{H}_{2} \mathrm{O}: \mathrm{HF}$ was prepared and $700 \mu \mathrm{L}$ of this solution was added to the reaction vessel. The resulting solution was allowed to stir at room temperature for 3 days. Once all the TIPS groups had been removed, methoxytrimethylsilane $(300 \mu \mathrm{L})$ was added and the crude mixture was stirred for $3 \mathrm{~h}$. The 
resulting solution was then concentrated in vacuo, adsorbed onto silica gel, and directly purified by flash column chromatography with $0 \% \rightarrow 10 \% \mathrm{MeOH}$ in $\mathrm{CH}_{2} \mathrm{Cl}_{2}$ to afford 19-hydroxysarmentogenin (2) (2.0 $\mathrm{mg}, 0.0049 \mathrm{mmol})$ in $42 \%$ yield over two steps, $\mathrm{R}_{\mathrm{f}}=0.3\left(15 \% \mathrm{MeOH}\right.$ in $\left.\mathrm{CH}_{2} \mathrm{Cl}_{2}\right) .{ }^{1} \mathrm{H}$ NMR $(700 \mathrm{MHz}$, $\mathrm{D}_{3} \mathrm{COD}$ ): $\delta 5.91$ (s, $1 \mathrm{H}, \mathrm{H} 22$ ), 5.02 (dd, $J=18.4,1.3 \mathrm{~Hz}, 1 \mathrm{H}, \mathrm{H} 21 \mathrm{~b}$ ), 4.92 (dd, $J=18.4,0.9 \mathrm{~Hz}, 1 \mathrm{H}, \mathrm{H} 21 \mathrm{a}$ ), $4.03(\mathrm{~m}, 1 \mathrm{H}, \mathrm{H3}$ ), 3.85 (ddd, J = 10.6, 4.2 Hz, 1H, H11), 3.77 (d, J = 11.1 Hz, 1H, H19b), 3.74 (d, J = 11.1 Hz, $1 \mathrm{H}, \mathrm{H} 19 \mathrm{a}), 2.90$ (dd, J = 9.0, $5.7 \mathrm{~Hz}, 1 \mathrm{H}, \mathrm{H} 17), 2.26-2.15$ (m, 3H, H1b, 15b, 16b), 2.07 (m, 1H, H5), 1.96 $1.70(\mathrm{~m}, 9 \mathrm{H}, \mathrm{H1a}, 2 \mathrm{~b}, 4 \mathrm{~b}, 6 \mathrm{~b}, 7 \mathrm{~b}, 8,9,15 \mathrm{a}, 16 \mathrm{a}), 1.67$ (dd, $J=13.2,4.2 \mathrm{~Hz}, 1 \mathrm{H}, \mathrm{H} 12 \mathrm{~b}), 1.53$ (dd, $J=13.2$, $10.5 \mathrm{~Hz}, 1 \mathrm{H}, \mathrm{H} 12 \mathrm{a}), 1.57-1.50$ (m, 1H, H2a), 1.39 - 1.20 (m, 3H, H4a, 6a, 7a), 0.91 (s, 3H, H18). ${ }^{13} \mathrm{C}$ NMR (175 MHz, D $\mathrm{C}_{3} \mathrm{COD}$ ): $\delta 177.7$ (C23), 177.1 (20), 118.0 (C22), 85.9 (C14), 75.3 (C21), 69.1 (C11), 67.6 (C3), 66.9 (C19), 51.9 (C17), 51.1 (C13), 50.6 (C12), 42.8, 41.6 (x2) (C10), 35.0, 33.5 (C15), 32.3 (C5), 29.2, 27.9 (C16), 27.7, 27.1, 22.3, 17.6 (C18). HRMS (ESI): Calculated for $\mathrm{C}_{23} \mathrm{H}_{35} \mathrm{O}_{6}{ }^{+}[\mathrm{M}+\mathrm{H}]^{+}$407.2428, found 407.2435. Calculated for $\mathrm{C}_{23} \mathrm{H}_{34} \mathrm{O}_{6} \mathrm{Na}^{+}[\mathrm{M}+\mathrm{Na}]^{+} 429.2248$, found 429.2255. IR (film, $\mathrm{cm}^{-1}$ ): 3315 (br), 2920, $1735,1632,1449,1022 .[\alpha]_{D}^{25}=+13.4(c=0.1, \mathrm{MeOH})$.

\section{Comparison to the reported 19-hydroxysarmentogenin ${ }^{1} \mathrm{H} N M R\left(700 \mathrm{MHz}, \mathrm{D}_{3} \mathrm{COD}\right)$ :}

\begin{tabular}{|c|c|c|}
\hline Position & $\begin{array}{c}\text { This work } \\
\delta_{\mathrm{H}} \text { (Multiplicity, } \mathrm{J} \text { in } \mathrm{Hz}, \text { No. of } \mathrm{H} \text { ) }\end{array}$ & $\begin{array}{c}\text { Inoue et al. }^{2} \\
\left.\delta_{\mathrm{H}} \text { (Multiplicity, } J \text { in } \mathrm{Hz}, \text { No. of } \mathrm{H}\right)\end{array}$ \\
\hline 22 & $5.91(\mathrm{~s}, 1 \mathrm{H})$ & $5.91(\mathrm{br} \mathrm{s}, 1 \mathrm{H})$ \\
\hline 21b & $5.02(\mathrm{dd}, J=18.4,1.3,1 \mathrm{H})$ & $5.02(\mathrm{dd}, J=18.3,1.8,1 \mathrm{H})$ \\
\hline $21 a$ & $4.92(\mathrm{dd}, J=18.4,0.9,1 \mathrm{H})$ & $4.91(\mathrm{dd}, J=18.3,1.2,1 \mathrm{H})$ \\
\hline 3 & $4.03(\mathrm{~m}, 1 \mathrm{H})$ & $4.03(\mathrm{~m}, 1 \mathrm{H})$ \\
\hline 11 & $3.85(\mathrm{ddd}, J=10.6,4.2,1 \mathrm{H})$ & $3.85(\mathrm{ddd}, J=10.9,3.5,1 \mathrm{H})$ \\
\hline $19 b$ & $3.77(\mathrm{~d}, J=11.1,1 \mathrm{H})$ & $3.77(\mathrm{~d}, J=12.0,1 \mathrm{H})$ \\
\hline $19 a$ & $3.74(\mathrm{~d}, J=11.1,1 \mathrm{H})$ & $3.74(\mathrm{~d}, J=12.0,1 \mathrm{H})$ \\
\hline 17 & $2.90(\mathrm{dd}, J=9.0,5.7,1 \mathrm{H})$ & $2.90(\mathrm{dd}, J=9.2,5.2,1 \mathrm{H})$ \\
\hline $1 b, 15 b, 16 b$ & $2.26-2.15(\mathrm{~m}, 3 \mathrm{H})$ & $2.15-2.26(\mathrm{~m}, 3 \mathrm{H})$ \\
\hline 5 & $2.07(\mathrm{~m}, 1 \mathrm{H})$ & $2.07(\mathrm{~m}, 1 \mathrm{H})$ \\
\hline $\begin{array}{c}H 1 a, 2 b, 4 b, 6 b \\
7 b, 8,9,15 a, 16 a\end{array}$ & $1.96-1.70(\mathrm{~m}, 9 \mathrm{H})$ & 1.69-1.96 (m, 9H) \\
\hline $12 b$ & $1.67(\mathrm{dd}, J=13.2,4.2,1 \mathrm{H})$ & $1.67(\mathrm{dd}, J=13.2,4.0,1 \mathrm{H})$ \\
\hline $12 a$ & $1.53(\mathrm{dd}, J=13.2,10.5,1 \mathrm{H})$ & $1.53(\mathrm{dd}, J=13.2,10.5,1 \mathrm{H})$ \\
\hline $2 a$ & $1.57-1.50(\mathrm{~m}, 1 \mathrm{H})$ & $1.50-1.58(\mathrm{~m}, 1 \mathrm{H})$ \\
\hline $4 a, 6 a, 7 a$ & $1.39-1.20(\mathrm{~m}, 3 \mathrm{H})$ & $1.20-1.39(\mathrm{~m}, 3 \mathrm{H})$ \\
\hline 18 & $0.91(\mathrm{~s}, 3 \mathrm{H})$ & $0.92(\mathrm{~s}, 3 \mathrm{H})$ \\
\hline
\end{tabular}


Comparison to the reported 19 -hydroxysarmentogenin ${ }^{13} \mathrm{C}$ NMR:

\begin{tabular}{|c|c|c|c|}
\hline Carbon \# & This work & Inoue et al. $^{2}$ & $\boldsymbol{\Delta}(\boldsymbol{\delta})$ \\
\hline $\mathbf{2 3}$ & 177.7 & 177.7 & 0.0 \\
\hline $\mathbf{2 0}$ & 177.1 & 177.1 & 0.0 \\
\hline $\mathbf{2 2}$ & 118.0 & 118.0 & 0.0 \\
\hline $\mathbf{1 4}$ & 85.9 & 85.9 & 0.0 \\
\hline $\mathbf{2 1}$ & 75.3 & 75.3 & 0.0 \\
\hline $\mathbf{1 1}$ & 69.1 & 69.1 & 0.0 \\
\hline $\mathbf{3}$ & 67.6 & 67.6 & 0.0 \\
\hline $\mathbf{1 9}$ & 66.9 & 66.9 & 0.0 \\
\hline $\mathbf{1 7}$ & 51.9 & 51.9 & 0.0 \\
\hline $\mathbf{1 3}$ & 51.1 & 51.1 & 0.0 \\
\hline $\mathbf{1 2}$ & 50.6 & 50.5 & 0.1 \\
\hline & 42.8 & 42.8 & 0.0 \\
\hline $\mathbf{1 0}$ & 41.6 & 41.6 & 0.0 \\
\hline & 35.0 & 35.0 & 0.0 \\
\hline $\mathbf{1 5}$ & 33.5 & 33.5 & 0.0 \\
\hline $\mathbf{5}$ & 32.3 & 32.3 & 0.0 \\
\hline & 29.2 & 29.2 & 0.0 \\
\hline $\mathbf{1 6}$ & 27.9 & 27.9 & 0.0 \\
\hline & 27.7 & 27.7 & 0.0 \\
\hline & 27.1 & 27.1 & 0.0 \\
\hline & 22.3 & 22.3 & 0.0 \\
\hline $\mathbf{1 8}$ & 17.6 & 17.6 & 0.0 \\
\hline
\end{tabular}

TLC of 19-hydroxysarmentogenin in $15 \% \mathrm{MeOH}$ in $\mathrm{CH}_{2} \mathrm{Cl}_{2}$ (stained with cerium ammonium molybdate):

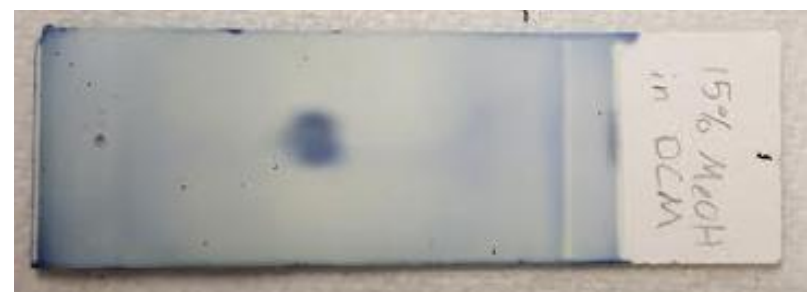



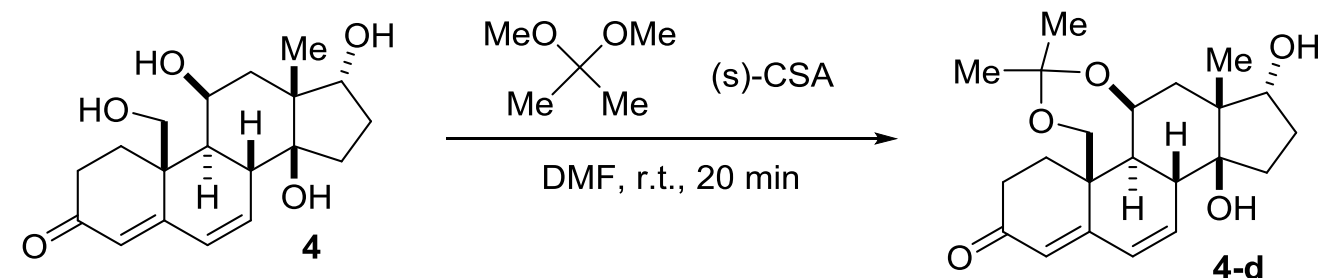

Compound 4-d. To a flame dried flask was added compound $4(50.0 \mathrm{mg}, 0.15 \mathrm{mmol}$ ) and Scamphorsulfonic acid (CSA) $(20.0 \mathrm{mg}, 0.086 \mathrm{mmol})$. The solids were solvated in $4.0 \mathrm{~mL}$ of dry dimethylformamide and 2,2-dimethoxypropane (550 uL) was added via syringe. The solution was allowed to stir at ambient temperature for 20 minutes when TLC indicated all starting material was consumed. The crude mixture was then diluted with EtOAc $(4.0 \mathrm{~mL})$ and quenched with a saturated solution of $\mathrm{NaHCO}_{3}(2.0 \mathrm{~mL})$ and Brine $(2.0 \mathrm{~mL})$. The organic layer was washed $3 x$ with a 1:1 mixture of water:brine (3.0 mL each). The organic layer was then dried with $\mathrm{Na}_{2} \mathrm{SO}_{4}$, filtered, and concentrated. The crude residue was then purified by flash column chromatography with $5 \% \rightarrow 10 \% \mathrm{MeOH}$ in $\mathrm{CH}_{2} \mathrm{Cl}_{2}$ to afford title compound 4-d $(38.6 \mathrm{mg}, 0.10 \mathrm{mmol})$ in $69 \%$ yield as a white solid, $\mathrm{R}_{\mathrm{f}}=0.5(10 \% \mathrm{MeOH}$ in $\mathrm{CH}_{2} \mathrm{Cl}_{2}$ ). ${ }^{1} \mathrm{H}$ NMR $\left(400 \mathrm{MHz}, \mathrm{CDCl}_{3}\right): \delta 6.51(\mathrm{dd}, J=9.9,2.1 \mathrm{~Hz}, 1 \mathrm{H}), 6.22(\mathrm{dd}, J=10.0,2.9 \mathrm{~Hz}, 1 \mathrm{H}), 5.78(\mathrm{~s}$, $1 \mathrm{H}), 4.27(\mathrm{~s}, 1 \mathrm{H}), 4.16(\mathrm{t}, J=8.8 \mathrm{~Hz}, 1 \mathrm{H}), 3.85(\mathrm{~d}, J=12.7 \mathrm{~Hz}, 1 \mathrm{H}), 3.32(\mathrm{dd}, J=12.7,1.6 \mathrm{~Hz}, 1 \mathrm{H}), 3.03(\mathrm{~d}, J$ $=11.0 \mathrm{~Hz}, 1 \mathrm{H}), 2.71-2.58(\mathrm{~m}, \mathrm{ovrlp}, 2 \mathrm{H}), 2.52-2.43(\mathrm{~m}, 1 \mathrm{H}), 2.23-2.10(\mathrm{~m}, 1 \mathrm{H}), 1.95-1.83(\mathrm{~m}, 1 \mathrm{H}), 1.68$ (dd, $J=14.6,3.4 \mathrm{~Hz}, 1 \mathrm{H}), 1.59-1.41\left(\mathrm{~m}\right.$, ovrlp, 4H), 1.36 (s, ovrlp, 4H), $1.33(\mathrm{~s}, 3 \mathrm{H}), 1.31(\mathrm{~s}, 3 \mathrm{H}) .{ }^{13} \mathrm{C}$ NMR $\left(100 \mathrm{MHz} \mathrm{CDCl}_{3}\right): \delta$ 199.7, 157.0, 137.9, 128.8, 126.0, 100.8, 83.1, 82.1, 65.4, 61.8, 48.1, 40.7, 38.8, 35.0, 34.0, 30.8, 29.6, 29.0, 25.4, 24.4, 21.2. IR (film, $\mathrm{cm}^{-1}$ ): 3346 (br), 2920, 1644, 1613, 1363, 1281, 1220,

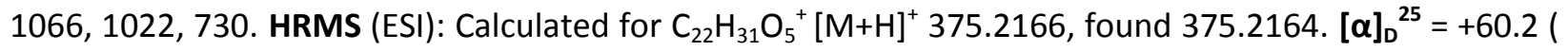
$\mathrm{c}=0.33, \mathrm{CH}_{2} \mathrm{Cl}_{2}$ ).
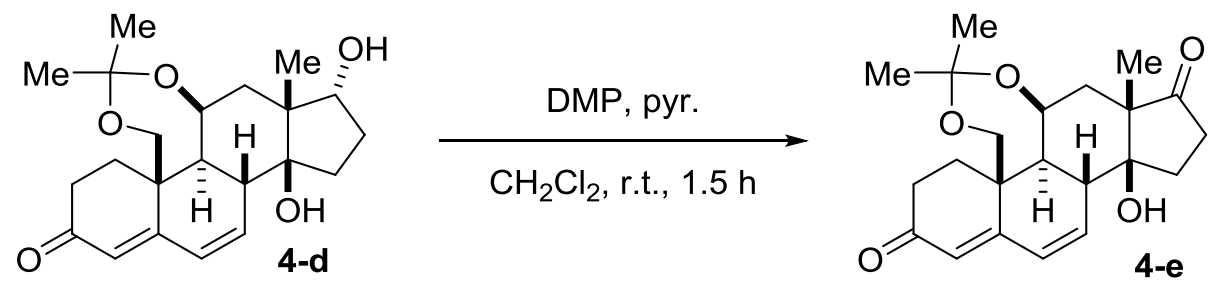

Compound 4-e. Cyclic ketal 4-d (28.0 mg, $0.075 \mathrm{mmol})$ and Dess-Martin periodinane $(140.0 \mathrm{mg}, 0.330$ mmol) were added successively to a dry flask and solvated in $3.0 \mathrm{~mL}$ of $\mathrm{CH}_{2} \mathrm{Cl}_{2}$ and $30.0 \mathrm{uL}$ of pyridine. The solution was allowed to stir at room temperature for 1.5 hours until all SM was consumed. If the reaction seems to stall by TLC, 1 drop of $\mathrm{H}_{2} \mathrm{O}$ can be added to promote further conversion to product. The solution was then quenched with saturated solutions of $\mathrm{Na}_{2} \mathrm{~S}_{2} \mathrm{O}_{3}(2.0 \mathrm{~mL})$ and $\mathrm{NaHCO}_{3}(1.5 \mathrm{~mL})$ and stirred at room temperature for 45 minutes. The organic layer was then extracted, dried with $\mathrm{Na}_{2} \mathrm{SO}_{4}$, filtered, and concentrated. The crude residue was then purified by flash column chromatography with $5 \% \rightarrow 10 \% \mathrm{MeOH}$ in $\mathrm{CH}_{2} \mathrm{Cl}_{2}$ to afford title compound 4-e $(25.0 \mathrm{mg}, 0.067 \mathrm{mmol})$ in $90 \%$ yield as a white solid, $\mathrm{R}_{\mathrm{f}}=0.7\left(10 \% \mathrm{MeOH}\right.$ in $\left.\mathrm{CH}_{2} \mathrm{Cl}_{2}\right) .{ }^{1} \mathrm{H}$ NMR $\left(700 \mathrm{MHz}, \mathrm{CDCl}_{3}\right): \delta 6.59$ (dd, J = 9.9, $2.1 \mathrm{~Hz}, 1 \mathrm{H}$ ), 6.28 (dd, $J=10.0,3.0 \mathrm{~Hz}, 1 \mathrm{H}), 5.80(\mathrm{~s}, 1 \mathrm{H}), 4.22(\mathrm{~s}, 1 \mathrm{H}), 3.85(\mathrm{~d}, J=12.8 \mathrm{~Hz}, 1 \mathrm{H}), 3.32(\mathrm{dd}, J=12.8,1.4 \mathrm{~Hz}, 1 \mathrm{H})$, $3.17(\mathrm{~d}, \mathrm{~J}=11.2 \mathrm{~Hz}, 1 \mathrm{H}), 2.67-2.59(\mathrm{~m}$, ovrlp, $2 \mathrm{H}), 2.54(\mathrm{~m}, \mathrm{ovrlp}, 3 \mathrm{H}), 2.03-1.96(\mathrm{~m}, 1 \mathrm{H}), 1.90$ - $1.79(\mathrm{~m}$, ovrlp, 2H), 1.69 (dd, $J=14.5,3.3 \mathrm{~Hz}, 1 \mathrm{H}$ ), $1.54-1.44$ (m, ovrlp, 2H), 1.37 (dd, J = 14.5, 2.7 Hz, $1 \mathrm{H}), 1.35$ (s, 3H), 1.33 (s, 3H), 1.29 (s, 3H). ${ }^{13} \mathrm{C}$ NMR (176 MHz, $\left.\mathrm{CDCl}_{3}\right): \delta 220.4,199.5,156.5,136.8,129.1,126.3$, 101.0, 81.6, 65.1, 61.6, 53.1, 47.7, 40.7, 38.1, 37.5, 33.9, 33.9, 30.7, 26.8, 25.3, 24.4, 16.9. IR (film, $\left.\mathrm{cm}^{-1}\right)$ : 
3448 (br), 2935, 1729, 1650, 1615, 1376, 1220, 1072, 881. HRMS(ESI): Calculated for $\mathrm{C}_{22} \mathrm{H}_{29} \mathrm{O}_{5}{ }^{+}[\mathrm{M}+\mathrm{H}]^{+}$ 373.2010, found 373.2006. $[\alpha]_{D}^{25}=+85.1\left(c=0.9, \mathrm{CH}_{2} \mathrm{Cl}_{2}\right)$.

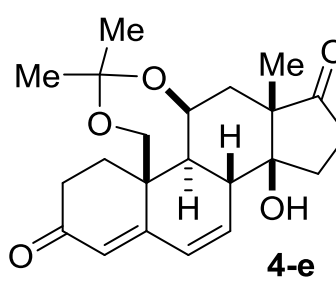

1) $\mathrm{Pd} / \mathrm{C}, \mathrm{H}_{2}$, EtOAc/MeOH/CH $\mathrm{Cl}_{2}$, r.t., $20 \mathrm{~min}$

2) $\mathrm{LiAl}(\mathrm{OtBu})_{3} \mathrm{H}, \mathrm{THF},-78$ to $-40^{\circ} \mathrm{C}, 2.5 \mathrm{~h}$

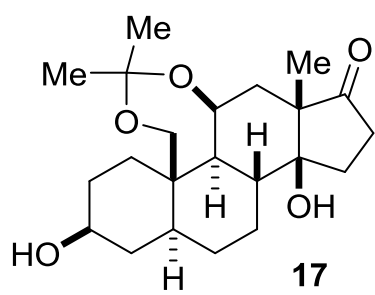

Panogenin Core 17. Compound 4-e $(14.0 \mathrm{mg}, 0.0376 \mathrm{mmol})$ and $10 \%$ Palladium on activated carbon (5.0 $\mathrm{mg}$ ) were added to a vial and solvated in $1.0 \mathrm{~mL}$ of a 3:1:1 mixture of EtOAc: $\mathrm{MeOH}: \mathrm{CH}_{2} \mathrm{Cl}_{2}$. The vial was purged with $\mathrm{N}_{2}$ and then with $\mathrm{H}_{2}$ and allowed to stir vigorously at room temperature under an atmosphere of $\mathrm{H}_{2}$. After 20 minutes, TLC showed complete conversion of starting material. The crude mixture was filtered through a plug of celite and the filtrate was concentrated. By NMR, the crude mixture was a 2.3:1 diastereomeric mixture of trans:cis A/B ring junctions. Without further purification, the residue was solvated in dry THF $(1.0 \mathrm{~mL})$ and cooled to $-78^{\circ} \mathrm{C}$ under a $\mathrm{N}_{2}$ atmosphere. Then, a solution of $\mathrm{LiAl}(\mathrm{OtBu})_{3} \mathrm{H}(22.0 \mathrm{mg}, 0.087 \mathrm{mmol})$ in THF $(0.50 \mathrm{~mL})$ was added slowly to the reaction vessel via syringe. The solution was then allowed to warm to $-40^{\circ} \mathrm{C}$ and was stirred at that temperature for 2.5 hours. Then, the reaction was quenched with a saturated solution of $\mathrm{NH}_{4} \mathrm{Cl}(0.50 \mathrm{~mL})$ and brine $(1.50$ $\mathrm{mL})$, and diluted with $\mathrm{CH}_{2} \mathrm{Cl}_{2}(2.0 \mathrm{~mL})$ as it was brought to room temperature. The aqueous layer was extracted $2 x$ with $\mathrm{CH}_{2} \mathrm{Cl}_{2}$ (2.0 mL each) and the combined organic layers were dried with $\mathrm{Na}_{2} \mathrm{SO}_{4}$, filtered, and concentrated. Crude NMR indicated a retention of 2.3:1 d.r., indicating completely stereoselective reduction of the $\mathrm{C} 3$ ketone. The crude residue was then purified by flash column chromatography with $66 \%$ EtOAc in hexanes to afford title compound $17(8.4 \mathrm{mg}, 0.022 \mathrm{mmol})$ in $59 \%$ yield over 2 steps as a white solid, $\mathrm{R}_{\mathrm{f}}=0.2$ (66\% EtOAc in hexanes). ${ }^{1} \mathrm{H}$ NMR (500 MHz, $\mathrm{CDCl}_{3}$ ): $\delta 4.11(\mathrm{~s}, 1 \mathrm{H}), 3.77$ (d, J= 12.5 $\mathrm{Hz}, 1 \mathrm{H}), 3.72$ - $3.64(\mathrm{~m}, 1 \mathrm{H}), 3.61(\mathrm{~d}, J=12.5 \mathrm{~Hz}, 1 \mathrm{H}), 2.46$ - $2.35(\mathrm{~m}, \mathrm{ovrlp}, 3 \mathrm{H}), 2.30-2.19(\mathrm{~m}, 1 \mathrm{H}), 2.17-$ $2.10(\mathrm{~m}, 1 \mathrm{H}), 2.10-1.99(\mathrm{~m}, 1 \mathrm{H}), 1.92-1.82(\mathrm{~m}, 2 \mathrm{H}), 1.74-1.67(\mathrm{~m}, 1 \mathrm{H}), 1.61-1.48(\mathrm{~m}, 2 \mathrm{H}), 1.44-1.35$ $(\mathrm{m}, 2 \mathrm{H}), 1.34-1.27$ (m, ovrlp, 8H), $1.24(\mathrm{~s}, 3 \mathrm{H}), 1.22-1.16(\mathrm{~m}$, ovrlp, $2 \mathrm{H}), 0.91(\mathrm{~d}, J=11.7 \mathrm{~Hz}, 1 \mathrm{H}), 0.70$ $(\mathrm{td}, J=13.3,3.4 \mathrm{~Hz}, 1 \mathrm{H}) .{ }^{13} \mathrm{C}$ NMR $\left(176 \mathrm{MHz}, \mathrm{CDCl}_{3}\right): \delta 221.5,100.5,82.9,71.1,65.1,57.8,53.1,51.1$, 43.0, 39.1, 37.8, 37.5, 36.9, 33.6, 33.0, 31.0, 27.6, 26.8, 25.5, 25.2, 24.2, 16.6. IR (film, cm $\mathrm{cm}^{-1}$ ): 3461 (br), 2928, 1730, 1457, 1379, 1278, 1218, 1134, 1080. HRMS(ESI): Calculated for $\mathrm{C}_{22} \mathrm{H}_{35} \mathrm{O}_{5}{ }^{+}[\mathrm{M}+\mathrm{H}]^{+} 379.2485$, found 379.2487. $[\alpha]_{D}^{25}=+18.9\left(\mathrm{c}=0.4, \mathrm{CHCl}_{3}\right)$. 

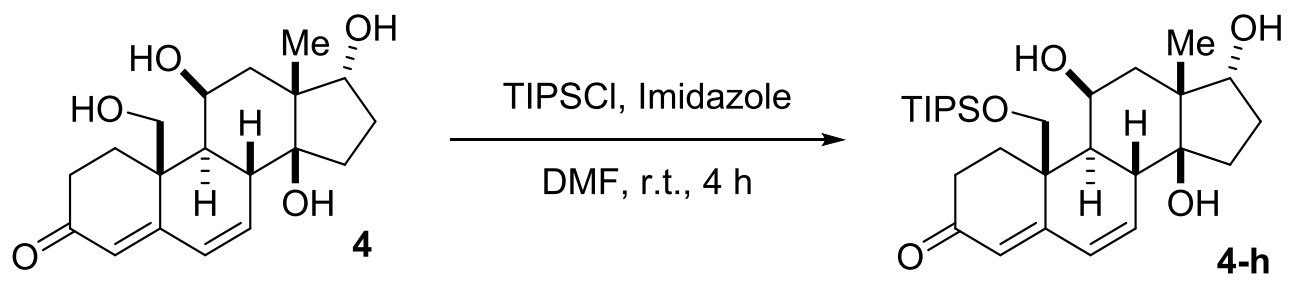

Compound 4-h. Tetra-ol 4 (250 mg, $0.75 \mathrm{mmol}$ ) and Imidazole (204 mg, $3.00 \mathrm{mml}$ ) was solvated in dry DMF $(5 \mathrm{~mL})$ at room temperature and triisopropylsilyl chloride $(316 \mu \mathrm{L}, 287 \mathrm{mg}, 1.5 \mathrm{mmol})$ was added. The reaction was allowed to stir for $4 \mathrm{~h}$ until the starting material was consumed. The solution was then quenched with $\mathrm{H}_{2} \mathrm{O}(3 \mathrm{~mL})$ and extracted $3 x$ with $\mathrm{CH}_{2} \mathrm{Cl}_{2}(15 \mathrm{~mL})$. The combined organic layers were washed with brine, dried over $\mathrm{Na}_{2} \mathrm{SO}_{4}$, filtered, and concentrated. The crude residue was directly purified by flash column chromatography with $40 \% \rightarrow 80 \%$ EtOAc in hexanes to afford title compound 4h $\left(295 \mathrm{mg}, 0.6 \mathrm{mmol}\right.$ ) in $81 \%$ yield as a colorless oil, $\mathrm{R}_{\mathrm{f}}=0.5$ (80\% EtOAc in hexanes). ${ }^{1} \mathbf{H} \mathbf{~ N M R}(700 \mathrm{MHz}$, $\left.\mathrm{CDCl}_{3}\right): \delta 6.49(\mathrm{dd}, J=9.8,1.4 \mathrm{~Hz}, 1 \mathrm{H}), 6.22(\mathrm{dd}, J=9.9,2.4 \mathrm{~Hz}, 1 \mathrm{H}), 5.75(\mathrm{~s}, 1 \mathrm{H}), 4.80(\mathrm{~s}, 1 \mathrm{H}), 4.20(\mathrm{~s}, 1 \mathrm{H})$, $4.16(\mathrm{t}, J=8.7 \mathrm{~Hz}, 1 \mathrm{H}), 3.90(\mathrm{~d}, J=11.0 \mathrm{~Hz}, 1 \mathrm{H}), 3.68(\mathrm{~d}, J=11.1 \mathrm{~Hz}, 1 \mathrm{H}), 2.80(\mathrm{~d}, J=11.2 \mathrm{~Hz}, 1 \mathrm{H}), 2.69-$ $2.61(\mathrm{~m}, 1 \mathrm{H}), 2.56-2.46$ (ovrlp, $2 \mathrm{H}$ ), $2.20-2.10(\mathrm{~m}, 1 \mathrm{H}), 1.88(\mathrm{dd}, J=14.8,3.1 \mathrm{~Hz}, 1 \mathrm{H}), 1.82$ (ddd, $J=$ 14.8, 4.6, $1 \mathrm{~Hz}, 1 \mathrm{H}$ ), 1.73 (td, $J=12.9,7.0 \mathrm{~Hz}, 1 \mathrm{H}$ ), 1.58 (d, $J=11.2 \mathrm{~Hz}, 1 \mathrm{H}$ ), $1.56-1.40$ (ovrlp, 4H), 1.37 (s, $3 \mathrm{H}), 1.31(\mathrm{~d}, J=14.8 \mathrm{~Hz}, 1 \mathrm{H}), 1.25(\mathrm{~d}, J=2.8 \mathrm{~Hz}, 1 \mathrm{H}), 1.21-1.13(\mathrm{~m}, 3 \mathrm{H}), 1.09(\mathrm{t}, J=7.9 \mathrm{~Hz}, 18 \mathrm{H}) .{ }^{13} \mathrm{C}$ NMR $\left(175 \mathrm{MHz}, \mathrm{CDCl}_{3}\right): \delta 198.6,158.8,139.3,128.9,124.6,83.5,82.2,63.2,62.5,51.5,47.2,42.0,39.5$, $34.4,33.5,29.4,28.5,27.8,20.2,18.0,17.9,11.9$. HRMS (ESI): Calculated for $\mathrm{C}_{28} \mathrm{H}_{46} \mathrm{O}_{5} \mathrm{SiNa}^{+}[\mathrm{M}+\mathrm{Na}]^{+}$ 513.3007, found 513.3011. IR (film, cm ${ }^{-1}$ ): 3419(br), 2942, 2866, 1728, 1646, 1616, 1463, 1369, 1244, $1188,1049,969,880,796,687 .[\alpha]_{D}{ }^{25}=+90.9\left(\mathrm{c}=0.5, \mathrm{CHCl}_{3}\right)$.
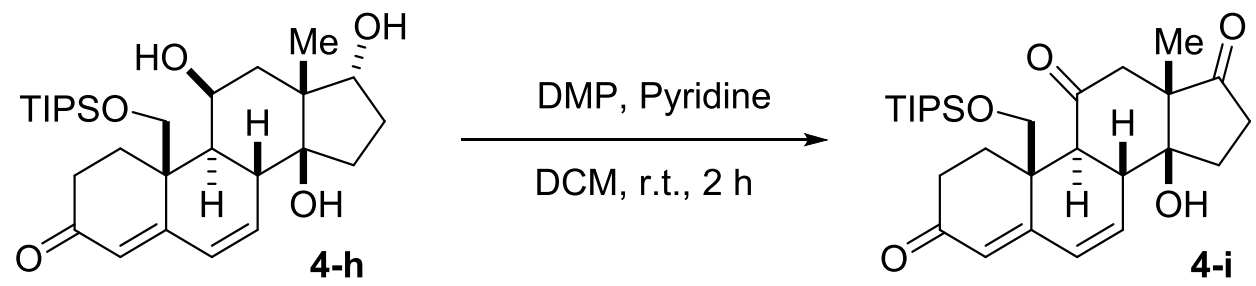

Compound 4-i. Compound 4-h (200 mg, $0.41 \mathrm{mmol}$ ) and the Dess-Martin periodinane (869 $\mathrm{mg}, 2.05$ mmol) was solvated in dry $\mathrm{CH}_{2} \mathrm{Cl}_{2}(8 \mathrm{~mL})$ and pyridine $(165 \mu \mathrm{L})$. The reaction mixture was allowed to stir at room temperature for $2 \mathrm{~h}$ until the starting material was consumed. The reaction was quenched with a saturated $\mathrm{Na}_{2} \mathrm{~S}_{2} \mathrm{O}_{3}$ solution $(3 \mathrm{~mL})$ and a saturated $\mathrm{NaHCO}_{3}$ solution $(3 \mathrm{~mL})$ and allowed to stir vigorously for $1 \mathrm{~h}$. The resulting mixture was extracted $3 x$ with $\mathrm{CH}_{2} \mathrm{Cl}_{2}\left(15 \mathrm{~mL}\right.$ each), dried over $\mathrm{Na}_{2} \mathrm{SO}_{4}$, filtered, adsorbed onto silica gel, and directly purified by flash column chromatography with $30 \% \rightarrow 40 \%$ $\rightarrow 50 \%$ EtOAc in hexanes to afford title compound 4-i (181 $\mathrm{mg}, 0.37 \mathrm{mmol}$ ) in $91 \%$ yield as a white solid, $\mathrm{R}_{\mathrm{f}}=0.8$ (80\% EtOAc in hexanes). ${ }^{1} \mathrm{H}$ NMR $\left(700 \mathrm{MHz}, \mathrm{CDCl}_{3}\right): \delta 6.50-6.32$ (ovrlp, 2H), $5.90(\mathrm{~s}, 1 \mathrm{H}), 4.36$ (d, $J=9.6 \mathrm{~Hz}, 1 \mathrm{H}), 4.16(\mathrm{~d}, J=9.6 \mathrm{~Hz}, 1 \mathrm{H}), 3.16(\mathrm{~d}, J=12.5,1 \mathrm{~Hz}, 1 \mathrm{H}), 2.87$ (ddd, $J=18.1,14.1,6.2 \mathrm{~Hz}, 1 \mathrm{H}$ ), 2.80 (dd, $J=13.2,5.7 \mathrm{~Hz}, 1 \mathrm{H}$ ), $2.64-2.52$ (ovrlp, 3H), $2.41-2.28$ (ovrlp, 3H), 2.12 - 1.97 (ovrlp, 3H), 1.69 (td, $J=13.8,5.8 \mathrm{~Hz}, 1 \mathrm{H}), 1.10(\mathrm{~s}, 3 \mathrm{H}), 1.05-0.96$ (ovrlp, 21H). ${ }^{13} \mathrm{C} \mathrm{NMR}\left(175 \mathrm{MHz}, \mathrm{CDCl}_{3}\right): \delta 215.9,211.7$, 204.7, 199.9, 155.9, 134.4, 131.3, 127.4, 80.0, 65.1, 56.7, 55.2, 47.2, 45.9, 40.6, 34.7, 33.2, 32.2, 26.9, 
18.0, 13.9, 11.8. HRMS (ESI): Calculated for $\mathrm{C}_{28} \mathrm{H}_{42} \mathrm{O}_{5} \mathrm{SiNa}^{+}[\mathrm{M}+\mathrm{Na}]^{+}$509.2694, found 509.2693. IR (film,

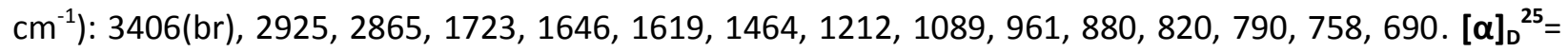
$+189.1\left(\mathrm{c}=1.25, \mathrm{CHCl}_{3}\right)$.

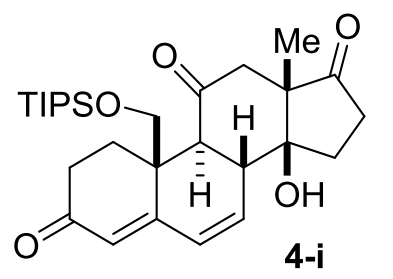
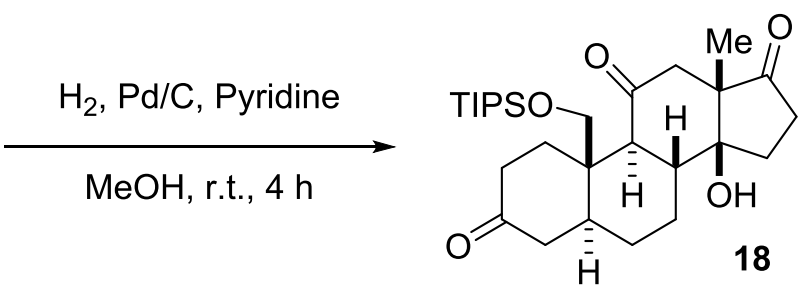

Compound 18. Triketone 4-i $(250 \mathrm{mg}, 0.514 \mathrm{mmol}$ ) and $10 \%$ palladium on activated carbon (109 $\mathrm{mg}$, $0.102 \mathrm{mmol}$ ) was solvated in $\mathrm{MeOH}(12 \mathrm{~mL})$ and pyridine $(90 \mu \mathrm{L})$ was added. The reaction flask was purged with $\mathrm{N}_{2}$, and then $\mathrm{H}_{2}$, and allowed to stir at room temperature for $4 \mathrm{~h}$. After consumption of the starting material, the reaction solution was filtered through a pad of celite with $\mathrm{MeOH}$ and the filtrate was concentrated. The resulting residue was purified by flash column chromatography with $30 \% \rightarrow 50 \%$ EtOAc in hexanes to afford title compound $18(210 \mathrm{mg}, 0.428 \mathrm{mmol})$ in $83 \%$ yield as a white solid, $R_{\mathrm{f}}=$ 0.7 (80\% EtOAc in hexanes). ${ }^{1} \mathrm{H}$ NMR $\left(700 \mathrm{MHz}, \mathrm{CDCl}_{3}\right): \delta 4.42(\mathrm{q}, J=10.6 \mathrm{~Hz}, 2 \mathrm{H}), 2.81(\mathrm{t}, J=13.5 \mathrm{~Hz}$, 1H), 2.75 (ddd, $J=13.5,7.3,2.0 \mathrm{~Hz}, 1 \mathrm{H}$ ), 2.67 (ddd, $J=16.0,12.9,7.4 \mathrm{~Hz}, 1 \mathrm{H}$ ), $2.56-2.47$ (ovrlp, 3H), 2.39 - 2.33 (ovrlp, $2 \mathrm{H}$ ), 2.29 (ddd, $J=16.0,3.9,1.9 \mathrm{~Hz}, 1 \mathrm{H}$ ), 2.21 (ddd, $J=15.5,3.9,1.7 \mathrm{~Hz}, 1 \mathrm{H}$ ), 2.15 (ddd, $J=$ 12.8, 6.5, 3.6 Hz, 1H), 2.08 - 2.00 (ovrlp, 3H), 1.86 (qd, J = 13.0, $3.9 \mathrm{~Hz}, 1 \mathrm{H}$ ), $1.67-1.59$ (m, $1 \mathrm{H}), 1.56$ (ddd, $J=12.2,6.3,3.2 \mathrm{~Hz}, 1 \mathrm{H}), 1.45(\mathrm{qd}, J=12.8,4.4 \mathrm{~Hz}, 1 \mathrm{H}), 1.37(\mathrm{~s}, 1 \mathrm{H}), 1.32-1.26(\mathrm{~m}, 1 \mathrm{H}), 1.19-1.11$ (m, 3H), 1.10 - 1.06 (ovrlp, 18H), 1.04 (s, 3H). $\left.{ }^{13} \mathrm{C} \mathrm{NMR} \mathrm{(175} \mathrm{MHz,} \mathrm{CDCl}\right): \delta 216.4,211.5,206.0,80.6$, 63.4, 59.8, 56.4, 47.1, 45.9, 44.9, 43.6, 39.5, 38.7, 35.3, 32.9, 27.8, 27.2, 26.4, 18.2, 18.2, 13.5, 11.9. HRMS (ESI): Calculated for $\mathrm{C}_{28} \mathrm{H}_{47} \mathrm{O}_{5} \mathrm{Si}^{+}[\mathrm{M}+\mathrm{H}]^{+}$491.3087, found 491.3191. IR (film, $\mathrm{cm}^{-1}$ ): 3462(br), 2923, $2865,1738,1704,1461,1382,1266,1209,1079,1064,957,882,799,733,683 .[\alpha]_{D}{ }^{25}=+28.6(c=0.5$, $\left.\mathrm{CHCl}_{3}\right)$.

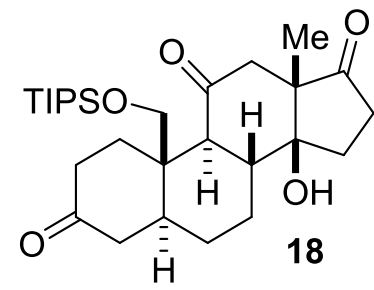
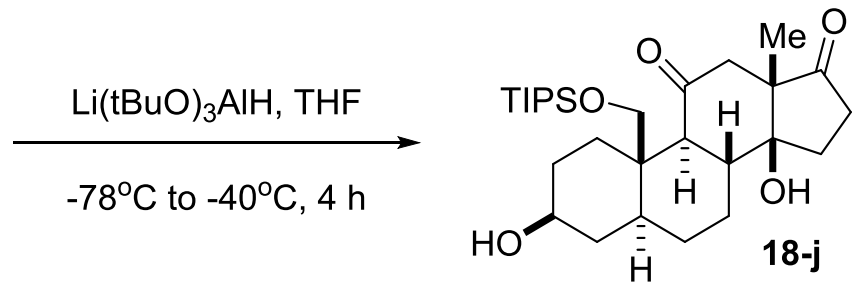

Compound 18-j. Compound 18 (160 mg, $0.326 \mathrm{mmol})$ was solvated in dry THF (10 mL) and cooled to $78^{\circ} \mathrm{C}$ under $\mathrm{N}_{2}$. Then, a solution of lithium tri-tert-butoxyaluminum hydride $(166 \mathrm{mg}, 0.653 \mathrm{mmol}$ ) in THF $(500 \mu \mathrm{L})$ was added dropwise via syringe. The reaction flask was allowed to warm to $-40^{\circ} \mathrm{C}$ and stirred for $4 \mathrm{~h}$ before it was quenched by the dropwise addition of a saturated $\mathrm{NH}_{4} \mathrm{Cl}$ solution. The mixture was then extracted $3 x$ with EtOAc ( $20 \mathrm{~mL}$ each), and the organic layers were washed with brine, dried over $\mathrm{Na}_{2} \mathrm{SO}_{4}$, filtered, and concentrated. The crude residue was then purified on flash column chromatography with $50 \% \rightarrow 70 \%$ EtOAc in hexanes to afford title compound 18-j (146 mg, $0.296 \mathrm{mmol}$ ) in $96 \%$ yield as a white solid, $\mathrm{R}_{\mathrm{f}}=0.5$ (80\% EtOAc in hexanes). ${ }^{1} \mathrm{H}$ NMR (700 MHz, $\mathrm{CDCl}_{3}$ ): $\delta 4.29$ (d, $J=$ $10.6 \mathrm{~Hz}, 1 \mathrm{H}$ ), $4.15(\mathrm{~d}, J=10.6 \mathrm{~Hz}, 1 \mathrm{H}), 3.60(\mathrm{tt}, J=10.5,5.2 \mathrm{~Hz}, 1 \mathrm{H}), 2.85(\mathrm{td}, J=12.3,3.6 \mathrm{~Hz}, 1 \mathrm{H}), 2.58-$ 
$2.46(\mathrm{~m}, 1 \mathrm{H}), 2.42$ (dd, $J=19.0,8.3 \mathrm{~Hz}, 1 \mathrm{H}), 2.32-2.20$ (ovrlp, 3H), 2.15 (d, $J=12.3,1 \mathrm{H}$ ), 2.07 (ddd, $J=$ 12.3, 6.3, $3.5 \mathrm{~Hz}, 1 \mathrm{H}$ ), $2.01-1.95(\mathrm{~m}, 1 \mathrm{H}), 1.91$ - 1.79 (ovrlp, 2H), 1.74 (d, $J=12.3 \mathrm{~Hz}, 1 \mathrm{H}), 1.71$ - $1.65(\mathrm{~m}$, $1 \mathrm{H}$ ), 1.54 (dd, $J=23.9,12.5 \mathrm{~Hz}, 1 \mathrm{H}$ ), $1.46-1.31$ (ovrlp, 3H), 1.18 (tt, $J=12.8,2.9 \mathrm{~Hz}, 1 \mathrm{H}$ ), $1.13-1.04$ (ovrlp, 21H), 1.03 (s, 3H), 0.91 (td, $J=14.0,4.0 \mathrm{~Hz}, 1 \mathrm{H}) .{ }^{13} \mathrm{C}$ NMR $\left(175 \mathrm{MHz}, \mathrm{CDCl}_{3}\right.$ ): $\delta$ 218.1, 207.1, 80.7, 70.8, 63.0, 60.1, 56.0, 47.5, 45.0, 42.9, 40.2, 37.6, 34.0, 33.4, 31.4, 27.9, 27.1, 26.4, 18.1, 14.8, 11.8. HRMS (ESI): Calculated for $\mathrm{C}_{28} \mathrm{H}_{49} \mathrm{O}_{5} \mathrm{Si}[\mathrm{M}+\mathrm{H}]^{+}$493.3344, found 493.3347. IR (film, cm${ }^{-1}$ ): 3407(br), 2925, $2866,1734,1458,1212,1083,884,801,761,687 .[\alpha]_{D}{ }^{25}=+28.4\left(c=0.75, \mathrm{CHCl}_{3}\right)$.
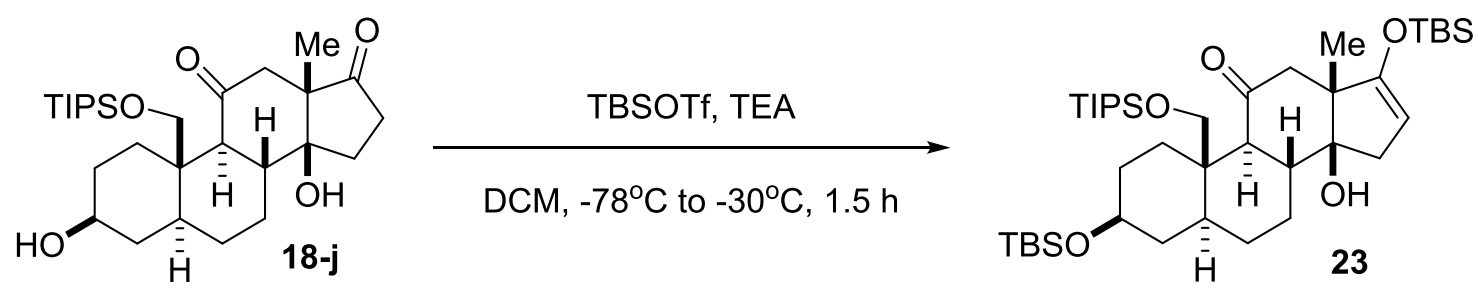

Compound 23. Diketone 18-j (120 mg, $0.243 \mathrm{mmol}$ ) was solvated in dry $\mathrm{CH}_{2} \mathrm{Cl}_{2}(8 \mathrm{~mL})$ and triethylamine $(683 \mu \mathrm{L})$ and cooled to $-78^{\circ} \mathrm{C}$ under a $\mathrm{N}_{2}$ atmosphere. Then, Tert-butyldimethylsilyl trifluoromethanesulfonate $(560 \mu \mathrm{L}, 644 \mathrm{mg}, 2.43 \mathrm{mmol})$ was added via syringe. The reaction was allowed to warm to $-30^{\circ} \mathrm{C}$ where it was stirred for $1.5 \mathrm{~h}$ until the reaction was complete. The solution was then quenched with a saturated $\mathrm{NaHCO}_{3}$ solution $(5 \mathrm{~mL})$ and extracted $3 x$ with $\mathrm{CH}_{2} \mathrm{Cl}_{2}(10 \mathrm{~mL}$ each), the combined organic layers were washed with brine, dried over $\mathrm{Na}_{2} \mathrm{SO}_{4}$, filtered, concentrated, and directly purified by flash column chromatography with $0 \% \rightarrow 5 \%$ EtOAc in hexanes to afford title compound 23 (152 mg, $0.211 \mathrm{mmol}$ ) in $87 \%$ yield as a white solid, $\mathrm{R}_{\mathrm{f}}=0.7$ (75\% EtOAc in hexanes). ${ }^{1} \mathbf{H}$ NMR $\left(700 \mathrm{MHZ}, \mathrm{C}_{6} \mathrm{D}_{6}\right): \delta 4.42(\mathrm{t}, J=2.0 \mathrm{~Hz}, 1 \mathrm{H}), 4.06(\mathrm{~d}, J=10.5 \mathrm{~Hz}, 1 \mathrm{H}), 3.97(\mathrm{~d}, J=10.5 \mathrm{~Hz}, 1 \mathrm{H}), 3.62$ (tt, $J=10.5,5.3 \mathrm{~Hz}, 1 \mathrm{H}), 3.03(\mathrm{~d}, J=12.5 \mathrm{~Hz}, 1 \mathrm{H}), 2.70(\mathrm{dt}, J=14.3,3.3 \mathrm{~Hz}, 1 \mathrm{H}), 2.65(\mathrm{dd}, J=16.5,2.0 \mathrm{~Hz}$, 1H), $2.58-2.45$ (ovrlp, 2H), $2.18-2.05(\mathrm{~m}, 1 \mathrm{H}), 1.98$ (dd, $J=16.5,2.4 \mathrm{~Hz}, 1 \mathrm{H}), 1.85(\mathrm{~d}, J=10.6 \mathrm{~Hz}, 1 \mathrm{H})$, $1.69-1.61(\mathrm{~m}, 1 \mathrm{H}), 1.58(\mathrm{ddt}, J=12.5,4.7,2.4 \mathrm{~Hz}, 1 \mathrm{H}), 1.47(\mathrm{~d}, J=12.6 \mathrm{~Hz}, 1 \mathrm{H}), 1.42(\mathrm{dd}, J=23.8,12.9$ $\mathrm{Hz}, 1 \mathrm{H}$ ), 1.35 (ddd, $J=17.0,13.4,4.2 \mathrm{~Hz}, 1 \mathrm{H}$ ), 1.24 (s, 3H), $1.23-1.18$ (ovrlp, 2H), $1.12(\mathrm{~s}, 1 \mathrm{H}$ ), $1.08-1.05$ (ovrlp, 18H), $1.04-0.97$ (ovrlp, 23H), $0.27(\mathrm{~s}, 3 \mathrm{H}), 0.18(\mathrm{~s}, 3 \mathrm{H}), 0.13(\mathrm{~d}, J=6.2 \mathrm{~Hz}, 3 \mathrm{H}), 0.12(\mathrm{~s}, 3 \mathrm{H}) .{ }^{13} \mathrm{C}$ NMR $\left(175 \mathrm{MHz}, \mathrm{C}_{6} \mathrm{D}_{6}\right): \delta$ 208.7, 156.1, 96.4, 79.9, 72.1, 60.9, 59.3, 53.6, 48.9, 45.0, 44.1, 42.1, 39.6, 38.5, $32.4,32.1,28.6,26.2,26.1,25.9,20.5,18.4,18.4,18.4,18.3,12.5,-4.2,-4.3,-4.4,-4.9$. HRMS (ESI): Calculated for $\mathrm{C}_{40} \mathrm{H}_{77} \mathrm{O}_{5} \mathrm{Si}_{3}[\mathrm{M}+\mathrm{H}]^{+}$721.5073, found 721.5078. IR (film, $\left.\mathrm{cm}^{-1}\right): 3545(\mathrm{br}), 2926,2856,1696$, $1657,1461,1334,1251,1193,1084,1065,872,834,776,679 .[\alpha]_{D}^{25}=-334.2\left(c=0.25, \mathrm{CHCl}_{3}\right)$.
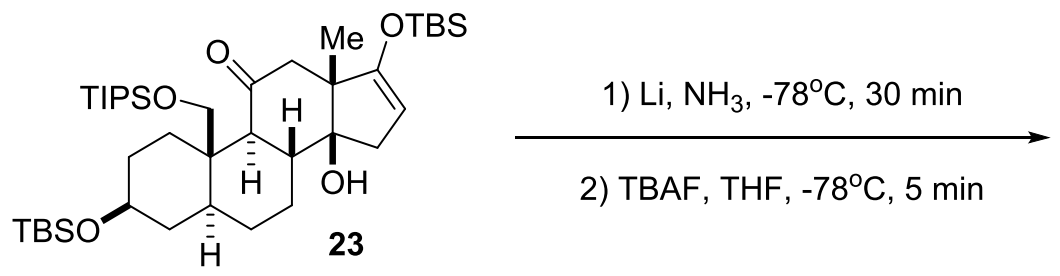

Compound 23-m. Gaseous ammonia $\left(\sim 8 \mathrm{~mL}\right.$ ) was condensed at $-78^{\circ} \mathrm{C}$ under $\mathrm{N}_{2}$ and lithium metal (74 $\mathrm{mg}, 10.62 \mathrm{mmol}$ ) was added and allowed to dissolve. After 30 minutes, Compound 23 (100 mg, 0.138 $\mathrm{mmol})$ dissolved in dry THF $(5.0 \mathrm{~mL})$ was slowly added to the deep blue reaction ammonia solution via syringe and allowed to stir at $-78^{\circ} \mathrm{C}$ for 30 minutes. Then, the reaction was quenched slowly by the dropwise addition of a saturated $\mathrm{NH}_{4} \mathrm{Cl}$ solution until the blue color had completely dissipated. The 
solution was allowed to warm to room temperature as the ammonia boiled off. The remaining mixture was extracted $3 x$ with EtOAc ( $15 \mathrm{~mL}$ each), dried over $\mathrm{Na}_{2} \mathrm{SO}_{4}$, filtered, and concentrated. The crude mixture was then solvated in dry THF $(6 \mathrm{~mL})$ and cooled to $-78^{\circ} \mathrm{C}$ under $\mathrm{N}_{2}$. $1 \mathrm{M}$ Tetrabutylammonium fluoride in THF ( $140 \mu \mathrm{L}, 0.140 \mathrm{mmol})$ was added via syringe and the reaction was stirred for precisely 5 minutes before being quenched with a saturated $\mathrm{NH}_{4} \mathrm{Cl}$ solution $(3 \mathrm{~mL})$ and warmed to room temperature. The crude mixture was then extracted $3 x$ with EtOAc ( $15 \mathrm{~mL}$ each), dried over $\mathrm{Na}_{2} \mathrm{SO}_{4}$, filtered, adsorbed onto silica gel, and directly purified by flash column chromatography with $10 \% \rightarrow 20 \%$ $\rightarrow 35 \%$ EtOAc in hexanes to afford title compound $23-\mathrm{m}(76 \mathrm{mg}, 0.125 \mathrm{mmol}$ ) in $91 \%$ yield as a white solid, $R_{f}=0.3$ (33\% EtOAc in hexanes).. ${ }^{1} \mathrm{H}$ NMR (700 MHz, CDCl $): \delta 4.01(\mathrm{~d}, J=10.7 \mathrm{~Hz}, 1 \mathrm{H}), 4.00-3.96$ $(\mathrm{m}, 1 \mathrm{H}), 3.94(\mathrm{~d}, J=10.5 \mathrm{~Hz}, 1 \mathrm{H}), 3.61(\mathrm{tt}, J=10.5,5.2 \mathrm{~Hz}, 1 \mathrm{H}), 3.39(\mathrm{~s}, 1 \mathrm{H}), 2.67(\mathrm{dt}, J=14.0,3.3 \mathrm{~Hz}, 1 \mathrm{H})$, $2.49-2.35(\mathrm{~m}, 2 \mathrm{H}), 2.22(\mathrm{dt}, J=13.6,10.0 \mathrm{~Hz}, 1 \mathrm{H}), 2.02-1.96(\mathrm{~m}, 1 \mathrm{H}), 1.85-1.78$ (ddd, $J=13.3,9.3,1.3$ $\mathrm{Hz}, 1 \mathrm{H}$ ), 1.74 - 1.65 (ovrlp, 2H), $1.61-1.57(\mathrm{~m}, 1 \mathrm{H}), 1.53$ (dd, J = 13.3, 3.5 Hz, 1H), 1.49 - 1.38 (ovrlp, 3H), $1.32(\mathrm{dd}, J=13.1,2.8 \mathrm{~Hz}, 1 \mathrm{H}), 1.26(\mathrm{tt}, J=14.0,7.0 \mathrm{~Hz}, 1 \mathrm{H}$ ), 1.23 - 1.14 (ovrlp, 5H), 1.14 - 1.10 (ovrlp, 21H), $1.09-1.06(\mathrm{~m}, 1 \mathrm{H}), 0.99(\mathrm{td}, J=14.1,3.3 \mathrm{~Hz}, 1 \mathrm{H}), 0.87(\mathrm{~s}, 9 \mathrm{H}), 0.03(\mathrm{~s}, 6 \mathrm{H}) .{ }^{13} \mathrm{C} \mathrm{NMR}(175 \mathrm{MHz}$, $\left.\mathrm{CDCl}_{3}\right): \delta 219.8,82.2,70.8,69.8,61.1,54.4,53.8,44.8,42.2,41.2,39.9,39.6,34.7,32.9,32.2,28.3,27.5$, 25.8, 25.6, 18.2, 18.1, 18.1, 14.3, 12.1, -4.5, -4.5. HRMS (ESI): Calculated for $\mathrm{C}_{34} \mathrm{H}_{64} \mathrm{O}_{5} \mathrm{Si}{ }_{2} \mathrm{Na}[\mathrm{M}+\mathrm{Na}]^{+}$ 631.4184, found 631.4187. IR (film, $\mathrm{cm}^{-1}$ ): 3457(br), 2930, 2866, 1733, 1461, 1374, 1256, 1091, 1060, $869,835,775,734,683 .[\alpha]_{D}^{25}=+7.2\left(c=1.0, \mathrm{CHCl}_{3}\right)$.

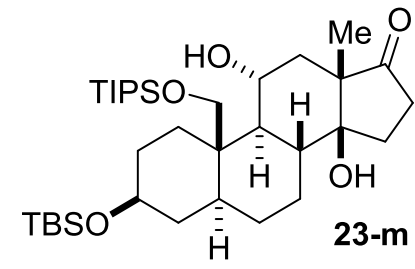

1) $\mathrm{H}_{2} \mathrm{NNH}_{2}-\mathrm{H}_{2} \mathrm{O}$, TEA, EtOH, $50^{\circ} \mathrm{C}, 6 \mathrm{~h}$

2) $\mathrm{I}_{2}$, TEA, THF, r.t., $1 \mathrm{~h}$

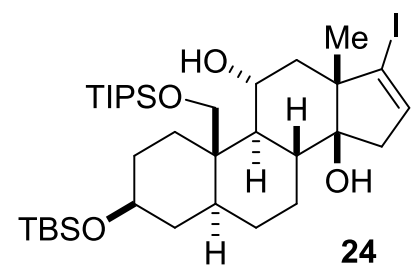

Compound 24. Compound $23-\mathrm{m}(100 \mathrm{mg}, 0.164 \mathrm{mmol})$ was solvated in EtOH $(16.0 \mathrm{~mL})$ and triethylamine $(461 \mu \mathrm{L})$. Hydrazine monohydrate $(164 \mathrm{mg}, 3.28 \mathrm{mmol})$ was then added and the solution was heated to $50^{\circ} \mathrm{C}$ and stirred for $6 \mathrm{~h}$ until all starting material was consumed. The resulting mixture was cooled to room temperature and concentrated in vacuo. The crude oil was then re-dissolved in THF $(16.0 \mathrm{~mL})$ and triethylamine $(461 \mu \mathrm{L})$. A solution of $\mathrm{I}_{2}(84 \mathrm{mg}, 0.656 \mathrm{mmol})$ in THF $(1 \mathrm{~mL})$ was prepared and $0.25 \mathrm{~mL}$ of this solution was added to the reaction vessel. After 10 minutes, additional $\mathrm{I}_{2}$ solution $(0.30 \mathrm{~mL})$ was added until a brown color persisted. The mixture was stirred at room temperature for 1 hour until it was complete. The solution was then quenched with a saturated $\mathrm{Na}_{2} \mathrm{~S}_{2} \mathrm{O}_{3}$ solution $(4 \mathrm{~mL})$ and a saturated $\mathrm{NaHCO}_{3}$ solution $(4 \mathrm{~mL})$ and stirred vigorously for 20 minutes. The resulting mixture was then extracted $3 x$ with EtOAc (15 mL each), dried over $\mathrm{Na}_{2} \mathrm{SO}_{4}$, filtered, adsorbed onto silica gel, and directly purified by flash column chromatography with $0 \% \rightarrow 5 \% \rightarrow 10 \% \rightarrow 20 \%$ EtOAc in hexanes to afford title compound 24 (98 mg, 0.136mmol) in 83\% yield, $R_{f}=0.8$ (33\% EtOAc in hexanes). ${ }^{1} \mathbf{H}$ NMR $\left(700 \mathrm{MHz}, \mathrm{CDCl}_{3}\right.$ ): $\delta 6.12(\mathrm{~s}, 1 \mathrm{H}), 4.00(\mathrm{~d}, J=10.5 \mathrm{~Hz}, 1 \mathrm{H}), 3.96(\mathrm{~d}, J=10.5 \mathrm{~Hz}, 1 \mathrm{H}), 3.85(\mathrm{~m}, 1 \mathrm{H}), 3.67-$ 3.53 (ovrlp, $2 \mathrm{H}$ ), 2.69 (dt, $J=14.0,3.3 \mathrm{~Hz}, 1 \mathrm{H}$ ), 2.60 (dd, $J=16.5,1.7 \mathrm{~Hz}, 1 \mathrm{H}$ ), 2.22 (dd, J=16.6, 3.0 Hz, $1 \mathrm{H}), 2.10(\mathrm{~m}, 1 \mathrm{H}), 1.91(\mathrm{dd}, J=13.2,3.1 \mathrm{~Hz}, 1 \mathrm{H}), 1.75-1.65$ (ovrlp, 3H), $1.59-1.57(\mathrm{~m}, 1 \mathrm{H}), 1.49-1.38$ (ovrlp, 2H), $1.29-1.23$ (ovrlp, 6H), $1.23-1.14$ (ovrlp, 4H), $1.15-1.10(\mathrm{~m}, 18 \mathrm{H}), 1.08-1.03(\mathrm{~m}, 1 \mathrm{H}), 0.98$ (ovrlp, 2H), 0.87 (s, 9H), 0.03 (s, 6H). ${ }^{13} \mathrm{C}$ NMR (175 MHz, $\mathrm{CDCl}_{3}$ ): $\delta 133.7,109.9,82.1,70.8,69.1,61.1$, 55.2, 54.6, 45.7, 44.9, 43.1, 41.6, 41.1, 39.7, 34.8, 32.2, 29.7, 28.3, 27.1, 25.9, 19.5, 18.2, 18.1, 18.1, 12.1, 


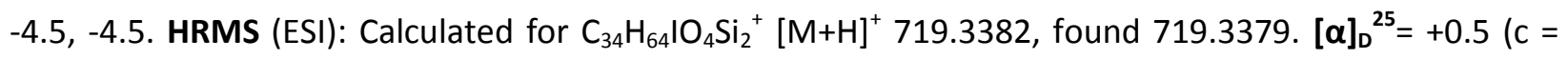
1.0, $\left.\mathrm{CHCl}_{3}\right)$.
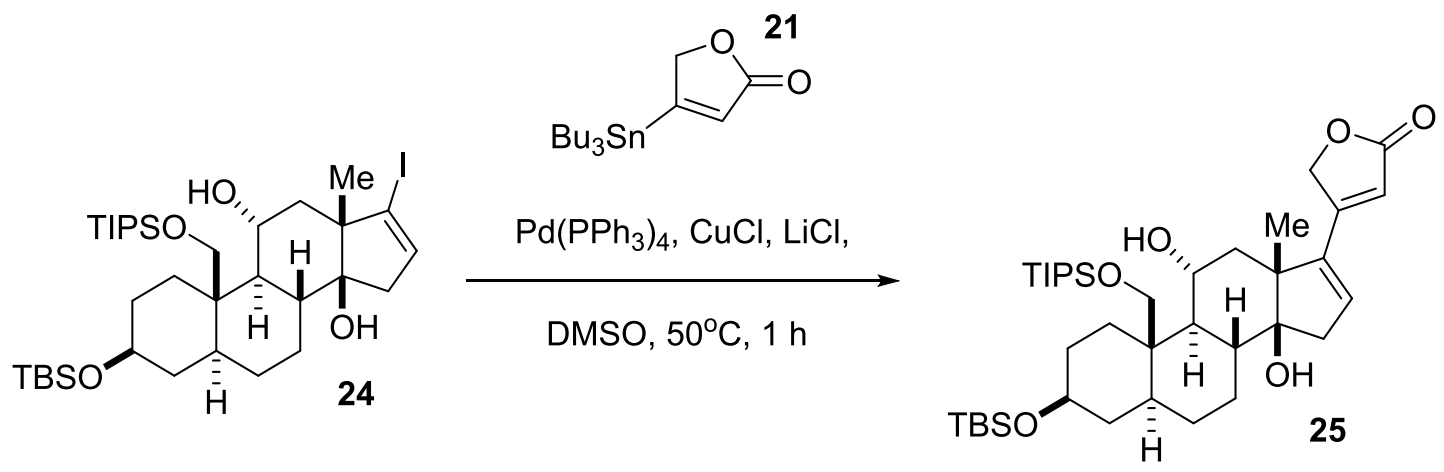

Compound 25. Vinyl lodide 24 (60 mg, $0.0835 \mathrm{mmol})$ along with $\mathrm{Pd}\left(\mathrm{PPh}_{3}\right)_{4}(19.4 \mathrm{mg}, 0.0167 \mathrm{mmol}), \mathrm{CuCl}$ (123 mg, $1.25 \mathrm{mmol})$, and $\mathrm{LiCl}(71 \mathrm{mg}, 1.67 \mathrm{mmol})$ was solvated in dry DMSO $(8 \mathrm{~mL})$ under a $\mathrm{N}_{2}$ atmosphere and stannyl butenolide $21(94 \mathrm{mg}, 0.25 \mathrm{mmol})$ was added via syringe. The reaction solution was heated to $50^{\circ} \mathrm{C}$ for 1 hour until the starting material was consumed. The solution was then quenched with $\mathrm{pH}=7$ phosphate buffer $(5 \mathrm{~mL})$ and extracted $3 x$ with EtOAc ( $15 \mathrm{~mL}$ each). The organic layers were combined, washed with brine, dried over $\mathrm{Na}_{2} \mathrm{SO}_{4}$, filtered, adsorbed onto silica gel, and directly purified by flash column chromatography with $0 \% \rightarrow 10 \% \rightarrow 20 \% \rightarrow 30 \%$ EtOAc in hexanes to afford title compound 25 (52 mg, $0.077 \mathrm{mmol})$ in 93\% yield, $\mathrm{R}_{\mathrm{f}}=0.3$ (50\% EtOAc in hexanes). ${ }^{1} \mathrm{H} \mathbf{~ N M R}$ $\left(700 \mathrm{MHz}, \mathrm{CDCl}_{3}\right): \delta 6.10(\mathrm{~s}, 1 \mathrm{H}), 5.99(\mathrm{~s}, 1 \mathrm{H}), 5.00(\mathrm{dd}, J=16.3,1.1 \mathrm{~Hz}, 1 \mathrm{H}), 4.91(\mathrm{dd}, J=16.2,1.0 \mathrm{~Hz}$, $1 \mathrm{H}), 4.02(\mathrm{~d}, J=10.5 \mathrm{~Hz}, 1 \mathrm{H}), 3.97(\mathrm{~d}, J=10.5 \mathrm{~Hz}, 1 \mathrm{H}), 3.94-3.86(\mathrm{~m}, 1 \mathrm{H}), 3.67(\mathrm{~d}, J=3.7 \mathrm{~Hz}, 1 \mathrm{H}), 3.61$ (tt, $J=10.5,5.2 \mathrm{~Hz}, 1 \mathrm{H}), 2.75(\mathrm{~d}, J=18.3 \mathrm{~Hz}, 1 \mathrm{H}), 2.69(\mathrm{dt}, J=14.0,3.3 \mathrm{~Hz}, 1 \mathrm{H}), 2.37(\mathrm{dd}, J=18.5,3.3 \mathrm{~Hz}$, 1H), $2.13-2.00$ (ovrlp, 2H), $1.75-1.67$ (ovrlp, 2H), $1.63-1.54$ (m, 1H), $1.52-1.38$ (ovrlp, 2H), 1.34 (s, 3H), 1.31 - 1.24 (ovrlp, 3H), $1.24-1.18(\mathrm{~m}, 3 \mathrm{H}$ ), $1.16-1.12$ (ovrlp, 18H), $1.08(\mathrm{~m}, 1 \mathrm{H}), 1.04$ (t, J = $10.5 \mathrm{~Hz}$, $1 \mathrm{H}), 0.98(\mathrm{td}, J=14.3,2.9 \mathrm{~Hz}, 1 \mathrm{H}), 0.87(\mathrm{~s}, 9 \mathrm{H}), 0.03(\mathrm{~s}, 6 \mathrm{H}) .{ }^{13} \mathrm{C} \mathrm{NMR}\left(175 \mathrm{MHz}, \mathrm{CDCl}_{3}\right): \delta 174.1,157.9$, 143.3, 131.9, 112.9, 85.2, 71.6, 70.8, 69.0, 61.1, 54.5, 52.6, 46.6, 44.8, 41.2, 41.1, 40.9, 39.7, 34.8, 32.2, 28.3, 27.2, 25.8, 18.2, 18.1, 18.0, 12.1, -4.5, -4.5. HRMS (ESI): Calculated for $\mathrm{C}_{38} \mathrm{H}_{66} \mathrm{O}_{6} \mathrm{Si}_{2} \mathrm{Na}^{+}[\mathrm{M}+\mathrm{Na}]^{+}$ 697.4290, found 697.4293. IR (film, $\mathrm{cm}^{-1}$ ): 3458 (br), 2922, 2852, 1738, 1618, 1461, 1376, 1255, 1093, $992,871,835,774,677 .[\alpha]_{D}^{25}=+28.3\left(\mathrm{c}=0.5, \mathrm{CHCl}_{3}\right)$.
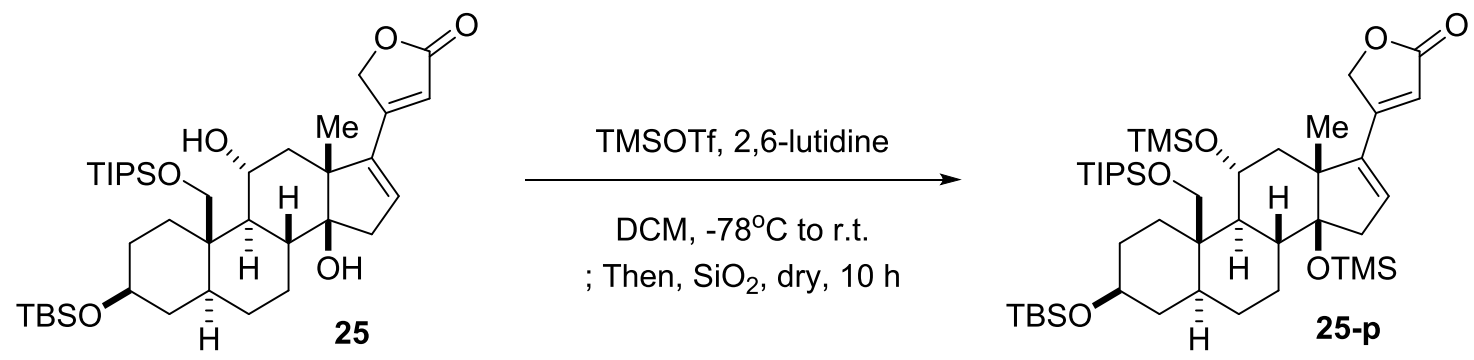

Compound 25-p. Compound $25(52 \mathrm{mg}, 0.077 \mathrm{mmol}$ ) and 2,6 - lutidine (180 $\mu \mathrm{L}, 165 \mathrm{mg}, 1.54 \mathrm{mmol})$ were solvated in dry $\mathrm{CH}_{2} \mathrm{Cl}_{2}(6 \mathrm{~mL})$ and cooled to $-78^{\circ} \mathrm{C}$ under a $\mathrm{N}_{2}$ atmosphere. Then, trimethylsilyl trifluoromethanesulfonate $(140 \mu \mathrm{L}, 171 \mathrm{mg}, 0.77 \mathrm{mmol})$ was added via syringe and the reaction was allowed to warm to room temperature. After $2 \mathrm{~h}$, the reaction was quenched with a saturated $\mathrm{NaHCO}_{3}$ 
solution $(2.5 \mathrm{~mL})$. The crude mixture was then extracted $3 x$ with EtOAc $\left(15 \mathrm{~mL}\right.$ each), dried over $\mathrm{Na}_{2} \mathrm{SO}_{4}$, filtered, and azeotroped $4 x$ with toluene $(5 \mathrm{~mL}$ each) to remove remaining 2,6-lutidine. The crude material was then solvated in EtOAc $(10 \mathrm{~mL})$ and adsorbed onto silica gel $(10 \mathrm{~g})$. The silica gel was allowed to sit at room temperature overnight. Then, the crude material was washed off the silica gel by filtering and eluting with $100 \%$ EtOAc. The filtrate was concentrated and directly purified by flash column chromatography with $0 \% \rightarrow 5 \%$ EtOAc in hexanes to afford title compound 25-p (50 mg, 0.061 $\mathrm{mmol}$ ) in 79\% yield, $\mathrm{R}_{\mathrm{f}}=0.7$ (33\% EtOAc in hexanes). ${ }^{1} \mathrm{H}$ NMR $\left(700 \mathrm{MHz}, \mathrm{CDCl}_{3}\right): \delta 6.04(\mathrm{t}, J=2.5 \mathrm{~Hz}, 1 \mathrm{H})$, $5.96(\mathrm{~s}, 1 \mathrm{H}), 5.00(\mathrm{dd}, J=16.2,1.4 \mathrm{~Hz}, 1 \mathrm{H}), 4.91(\mathrm{dd}, J=16.1,1.3 \mathrm{~Hz}, 1 \mathrm{H}), 4.33-4.23(\mathrm{~m}, 1 \mathrm{H}), 4.09(\mathrm{~d}, J=$ $10.8 \mathrm{~Hz}, 1 \mathrm{H}), 3.89(\mathrm{~d}, J=10.8 \mathrm{~Hz}, 1 \mathrm{H}), 3.58(\mathrm{tt}, J=10.5,5.2 \mathrm{~Hz}, 1 \mathrm{H}), 2.92(\mathrm{~d}, J=18.4 \mathrm{~Hz}, 1 \mathrm{H}), 2.46-2.33$ (ovrlp, 2H), 2.15 - 1.98 (ovrlp, 2H), 1.85 (dd, $J=14.0,3.1 \mathrm{~Hz}, 1 \mathrm{H}$ ), 1.67 (d, $J=13.0 \mathrm{~Hz}, 1 \mathrm{H}$ ), $1.58(\mathrm{~m}, 1 \mathrm{H}$ ), 1.53 - 1.51 (ovrlp, 2H), 1.48 - $1.36(\mathrm{~m}, 2 \mathrm{H}), 1.34-1.20$ (ovrlp, 3H), 1.15 (s, 3H), 1.14 - 1.06 (ovrlp, 22H), $1.06-0.98(\mathrm{~m}, 1 \mathrm{H}), 0.95-0.90(\mathrm{~m}, 1 \mathrm{H}), 0.88(\mathrm{~s}, 9 \mathrm{H}), 0.04(\mathrm{~s}, 6 \mathrm{H}), 0.00(\mathrm{~s}, 9 \mathrm{H}),-0.01(\mathrm{~s}, 9 \mathrm{H}) .{ }^{13} \mathrm{C} \mathrm{NMR}(175$ $\left.\mathrm{MHz}, \mathrm{CDCl}_{3}\right): \delta 174.7,158.6,142.7,134.0,111.5,88.3,71.8,71.7,67.6,62.6,58.1,52.6,45.6,43.7,40.7$, 40.3, 40.0, 39.1, 33.6, 32.3, 29.7, 28.9, 28.1, 25.9, 20.7, 18.3, 18.3, 18.2, 12.1, 2.5, 0.9, -4.5. HRMS (ESI): Calculated for $\mathrm{C}_{44} \mathrm{H}_{82} \mathrm{O}_{6} \mathrm{Si}_{4} \mathrm{Na}^{+}[\mathrm{M}+\mathrm{Na}]^{+}$841.5081, found 841.5092. IR (film, $\left.\mathrm{cm}^{-1}\right)$ : 2926, 2861, 1784, 1754, $1625,1463,1250,1155,1086,1006,836,774,681 .[\alpha]_{D}^{25}=+24.0\left(c=0.2, \mathrm{CHCl}_{3}\right)$.
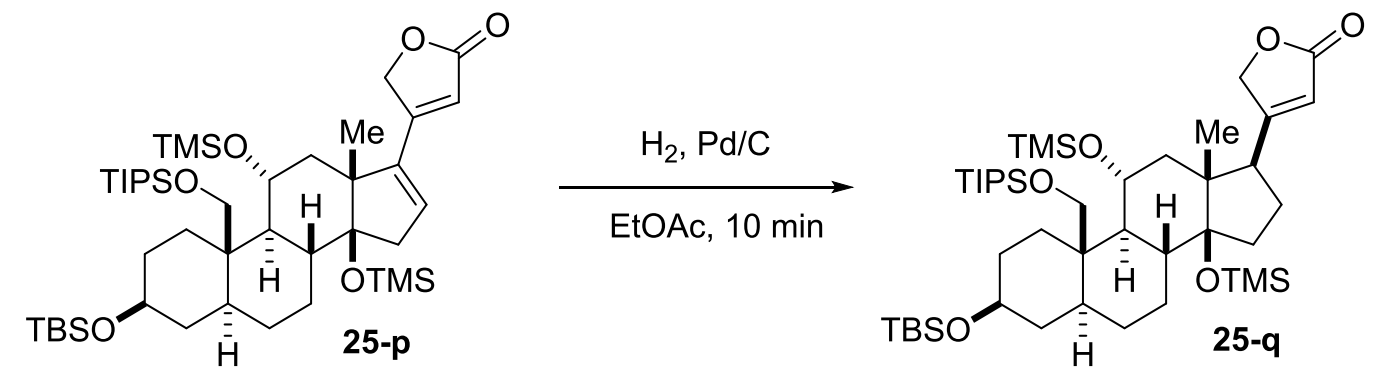

Compound 25-q. Compound 25-p (42.2 mg, $0.0513 \mathrm{mmol}$ ) and $10 \%$ Palladium on activated carbon (21.6 $\mathrm{mg}$ ) was solvated in EtOAc ( $8 \mathrm{~mL}$ ) and the reaction vessel was purged with $\mathrm{N}_{2}$, and then with $\mathrm{H}_{2}$, and the solution was allowed to stir vigorously under an atmosphere of $\mathrm{H}_{2}$. After 10 minutes, all starting material was consumed. The reaction solution was then filtered through a pad of celite and silica gel with $100 \%$ EtOAc. $2 / 3$ rds of the resulting crude filtrate was purified by semi-preparative HPLC with $10 \%$ EtOAc in hexanes to afford title compound $\mathbf{2 5 - q}(15.1 \mathrm{mg}, 0.018 \mathrm{mmol})$ in $54 \%$ yield as a colorless liquid, $\mathrm{R}_{\mathrm{f}}=0.7$ (33\% EtOAc in hexanes), and its C17 epimer (25-q-epi) $(6.0 \mathrm{mg}, 0.0073 \mathrm{mmol})$ in $22 \%$ yield, $R_{\mathrm{f}}=0.7$ (33\% EtOAc in hexanes).. [25-q]: ${ }^{1} \mathrm{H}$ NMR $\left(700 \mathrm{MHz}, \mathrm{CDCl}_{3}\right): \delta 5.83(\mathrm{~d}, J=1.0 \mathrm{~Hz}, 1 \mathrm{H}), 4.80$ (dd, $J=17.3,1.6 \mathrm{~Hz}$, $1 \mathrm{H}), 4.70(\mathrm{dd}, J=16.7,1.7 \mathrm{~Hz}, 1 \mathrm{H}), 4.47(\mathrm{dd}, J=9.1,4.4 \mathrm{~Hz}, 1 \mathrm{H}), 3.93(\mathrm{~d}, J=10.8 \mathrm{~Hz}, 1 \mathrm{H}), 3.88(\mathrm{~d}, J=10.8$ $\mathrm{Hz}, 1 \mathrm{H}), 3.60(\mathrm{tt}, J=10.5,5.2 \mathrm{~Hz}, 1 \mathrm{H}), 3.31(\mathrm{dd}, J=9.8,7.8 \mathrm{~Hz}, 1 \mathrm{H}), 2.37(\mathrm{dt}, J=10.3,3.4 \mathrm{~Hz}, 1 \mathrm{H}), 2.09(\mathrm{td}$, $J=12.8,7,0 \mathrm{~Hz}, 1 \mathrm{H}$ ), $2.04-1.96(\mathrm{~m}, 1 \mathrm{H}), 1.89-1.77$ (ovrlp, 2H), 1.71 - 1.68 (ovrlp, 3H), 1.65 (dd, $J=14.3$, $3.6 \mathrm{~Hz}, 1 \mathrm{H}$ ), $1.53-1.48(\mathrm{~m}, 2 \mathrm{H}), 1.46$ (dd, $J=14.3,5.6 \mathrm{~Hz}, 1 \mathrm{H}$ ), 1.30 (ovrlp, 4H), 1.17 (td, $J=12.2,3.6 \mathrm{~Hz}$, 1H), 1.16 - 1.04 (ovrlp, 22H), $1.03-0.94(\mathrm{~m}, 1 \mathrm{H}), 0.88(\mathrm{~s}, 9 \mathrm{H}), 0.73(\mathrm{~s}, 3 \mathrm{H}), 0.12(\mathrm{~s}, 9 \mathrm{H}), 0.09(\mathrm{~s}, 9 \mathrm{H}), 0.04$ $(\mathrm{s}, 6 \mathrm{H}) .{ }^{13} \mathrm{C}$ NMR $\left(175 \mathrm{MHz}, \mathrm{CDCl}_{3}\right): \delta 174.2,172.8,116.2,90.0,73.8,71.6,68.0,61.4,58.2,48.7,48.7$, 45.5, 40.7, 40.2, 39.0, 33.3, 32.2, 32.0, 29.3, 28.5, 27.3, 25.9, 21.7, 18.3, 18.2, 18.2, 12.1, 2.9, 1.2, -4.5, 4.5. HRMS (ESI): Calculated for $\mathrm{C}_{44} \mathrm{H}_{84} \mathrm{O}_{6} \mathrm{Si}_{4} \mathrm{Na}^{+}[\mathrm{M}+\mathrm{Na}]^{+}$843.5237, found 843.5232. IR (film, $\left.\mathrm{cm}^{-1}\right): 2948$, $2867,1782,1753,1458,1250,1084,887,836,772 .[\alpha]_{D}^{25}=+14.9\left(c=0.5, \mathrm{CHCl}_{3}\right)$.

C17 epimer [25-q-epi]: ${ }^{1} \mathbf{H}$ NMR $\left(700 \mathrm{MHz}, \mathrm{CDCl}_{3}\right): \delta 5.87(\mathrm{~d}, J=1.2 \mathrm{~Hz}, 1 \mathrm{H}), 4.81$ (dd, $J=17.5,1.7 \mathrm{~Hz}$, $1 \mathrm{H}), 4.70(\mathrm{dd}, J=17.6,1.2 \mathrm{~Hz}, 1 \mathrm{H}), 4.19(\mathrm{td}, J=10.6,4.3 \mathrm{~Hz}, 1 \mathrm{H}), 4.17(\mathrm{~d}, J=14 \mathrm{~Hz}, 1 \mathrm{H}), 3.88(\mathrm{~d}, J=11.0$ $\mathrm{Hz}, 1 \mathrm{H}), 3.55(\mathrm{tt}, J=10.6,5.6 \mathrm{~Hz}, 1 \mathrm{H}), 2.91(\mathrm{t}, J=9.7 \mathrm{~Hz}, 1 \mathrm{H}), 2.42(\mathrm{dt}, J=14.2,3.3 \mathrm{~Hz}, 1 \mathrm{H}), 2.24(\mathrm{td}, J=$ 
12.1, 3.7 Hz, 1H), 2.11 (ddd, $J=14.5,12.1,5.6 \mathrm{~Hz}, 1 \mathrm{H}$ ), 2.03 - 1.91 (ovrlp, $2 \mathrm{H}$ ), 1.82 - 1.65 (ovrlp, 3H), 1.64 - $1.58(\mathrm{~m}, 1 \mathrm{H}$ ), 1.53 - 1.51 (ovrlp, 2H), 1.41 - $1.32(\mathrm{~m}, 1 \mathrm{H}$ ), 1.28 - 1.25 (ovrlp, 2H), 1.23 - 1.12 (ovrlp, 5H), 1.13 - 1.08 (ovrlp, 18H), 1.04 (ovrlp, 2H), $1.01(\mathrm{~s}, 3 \mathrm{H}), 1.00-0.94(\mathrm{~m}, 1 \mathrm{H}), 0.87(\mathrm{~s}, 9 \mathrm{H}), 0.12(\mathrm{~s}, 9 \mathrm{H}), 0.05$ (9s, H), $0.04(\mathrm{~d}, J=1.7 \mathrm{~Hz}, 6 \mathrm{H}) .{ }^{13} \mathrm{C}$ NMR $\left(175 \mathrm{MHz}, \mathrm{CDCl}_{3}\right): \delta 173.7,171.1,116.6,90.2,73.7,71.6,69.7$, 63.9, 56.1, 50.2, 49.0, 45.4, 42.0, 41.0, 40.8, 39.4, 35.7, 32.7, 30.1, 29.7, 29.1, 28.3, 25.9, 24.6, 20.0, 18.4, 18.4, 18.2, 12.0, 2.6, 1.3, -4.5, -4.5. IR (film, $\left.\mathrm{cm}^{-1}\right)$ : 2950, 2866, 1783, 1752, 1458, 1250, 1084, 886, 838, 677. $\mathrm{HRMS}(\mathrm{ESI})$ : calculated for $\mathrm{C}_{44} \mathrm{H}_{84} \mathrm{O}_{6} \mathrm{Si}_{4} \mathrm{Na}^{+}[\mathrm{M}+\mathrm{Na}]^{+} 843.5237$, found 843.5242. $[\alpha]_{D}{ }^{25}=-28.8(\mathrm{c}=$ $0.25, \mathrm{CHCl} 3)$.
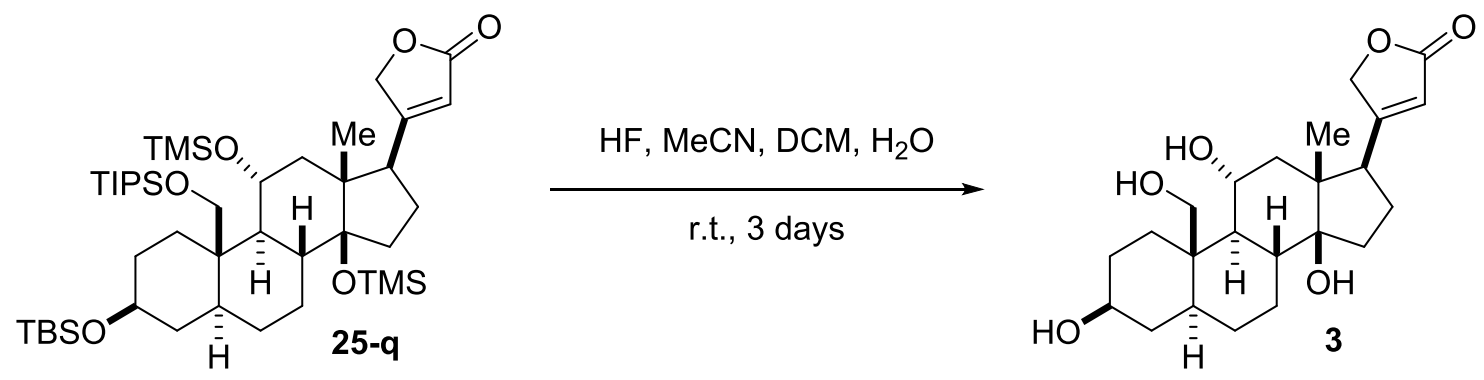

Trewianin aglycone (3). Compound $25-\mathrm{q}(10.0 \mathrm{mg}, 0.01219 \mathrm{mmol})$ was solvated in $1 \mathrm{~mL}$ of $1: 1$ $\mathrm{CH}_{2} \mathrm{Cl}_{2}: \mathrm{MeCN}$. Then, a stock solution of 18:3:1 MeCN: $\mathrm{H}_{2} \mathrm{O}: \mathrm{HF}$ was prepared and $900 \mu \mathrm{L}$ of this solution was added to the reaction vessel. The resulting solution was allowed to stir at room temperature for 3 days. Once all the TIPS groups had been removed, methoxytrimethylsilane $(1 \mathrm{~mL})$ was added and the crude mixture was stirred for 30 minutes. The resulting solution was then concentrated in vacuo, adsorbed onto silica gel, and directly purified by flash column chromatography with $0 \% \rightarrow 10 \% \mathrm{MeOH}$ in $\mathrm{CH}_{2} \mathrm{Cl}_{2}$ to afford Trewianin aglycone (3) $(4.5 \mathrm{mg}, 0.011 \mathrm{mmol})$ in $91 \%$ yield, $\mathrm{R}_{\mathrm{f}}=0.2(10 \% \mathrm{MeOH}$ in $\mathrm{CH}_{2} \mathrm{Cl}_{2}$ ). ${ }^{1} \mathrm{H}$ NMR $\left(700 \mathrm{MHz}, \mathrm{D}_{5}\right.$-Pyridine): $\delta 6.28(\mathrm{t}, \mathrm{J}=4.0 \mathrm{~Hz}, 1 \mathrm{H}, \mathrm{C} 19-\mathrm{OH}), 6.12(\mathrm{~s}, 1 \mathrm{H}, \mathrm{H} 22), 6.02(\mathrm{~d}, J=$ $4.2 \mathrm{~Hz}, 1 \mathrm{H}, \mathrm{C} 3-\mathrm{OH}$ ), $5.52-5.40$ (ovrlp, $2 \mathrm{H}, \mathrm{C} 11-\mathrm{OH}, \mathrm{C} 14-\mathrm{OH}$ ), 5.30 (dd, J = 18.0, 1H, $1.6 \mathrm{~Hz}, \mathrm{H} 21 \mathrm{a}$ ), 5.04 (dd, $J=18.0,1.5 \mathrm{~Hz}, 1 \mathrm{H}, \mathrm{H} 21 \mathrm{~b}$ ), $4.65-4.54(\mathrm{~m}, 1 \mathrm{H}, \mathrm{H} 11), 4.30$ (dd, $J=11.2,4.3 \mathrm{~Hz}, 1 \mathrm{H}, \mathrm{H} 19 \mathrm{a}), 4.24$ (dd, $J$ $=11.2,3.4 \mathrm{~Hz}, 1 \mathrm{H}, \mathrm{H} 19 \mathrm{~b}), 4.10-4.00(\mathrm{~m}, 1 \mathrm{H}, \mathrm{H3}), 3.53(\mathrm{dt}, J=13.9,3.4 \mathrm{~Hz}, 1 \mathrm{H}, \mathrm{H1a}), 2.94(\mathrm{dd}, J=9.4,5.2$ $\mathrm{Hz}, 1 \mathrm{H}, \mathrm{H} 17), 2.45(\mathrm{~m}, 1 \mathrm{H}, \mathrm{H7a}), 2.30$ (dt, $J=12.2,9.5 \mathrm{~Hz}, 1 \mathrm{H}, \mathrm{H} 15 \mathrm{a}), 2.25-2.20(\mathrm{~m}, 1 \mathrm{H}, \mathrm{H} 2 \mathrm{a}), 2.17(\mathrm{td}, J=$ 11.7, 3.8 Hz, 1H, H8), 2.11 - 2.08 (ovrlp, 2H, H7b, 16a), 2.04 - 1.94 (ovrlp, 4H, H4a, 12a, 15b, 16b), 1.92 1.84 (ovrlp, 2H, H4b, 12b), 1.45 (t, J = $10.7 \mathrm{~Hz}, 1 \mathrm{H}, \mathrm{H}$ ), $1.42-1.30$ (ovrlp, 5H, H1b, 2b, 5, 6a, 6b), 1.16 (s, $3 \mathrm{H}, \mathrm{H} 18) .{ }^{13} \mathrm{C}$ NMR (175 MHz, $\mathrm{D}_{5}$-Pyridine): $\delta$ 175.6(C23), 174.5(C20), 117.8(C22), 84.7(C14), 73.8(C21), 70.4(C3), 70.2(C11), 59.5(C19), 55.5(C9), 51.4(C17), 50.6(C13), 50.0(C12), 45.6(C5), 42.2(C8), 41.6(C10), 40.3(C4), 35.8(C1), 33.6(C15), 33.1(C2), 29.5(C6), 28.3(C7), 27.5(C16), 18.0(C18). HRMS (ESI): Calculated for $\mathrm{C}_{23} \mathrm{H}_{34} \mathrm{O}_{6} \mathrm{Na}^{+}[\mathrm{M}+\mathrm{Na}]^{+} 429.2248$, found 429.2253. IR (film, $\mathrm{cm}^{-1}$ ): 3358(br), 2926, 2856, 1736, 1623, $1448,1375,1172,1035,961,696$. [ $\alpha]_{D}^{25}=+9.9\left(\mathrm{c}=0.25, \mathrm{CH}_{3} \mathrm{OH}\right)$.

TLC of Trewianin aglycone in $10 \% \mathrm{MeOH}$ in $\mathrm{CH}_{2} \mathrm{Cl}_{2}$ (stained with cerium ammonium molybdate):

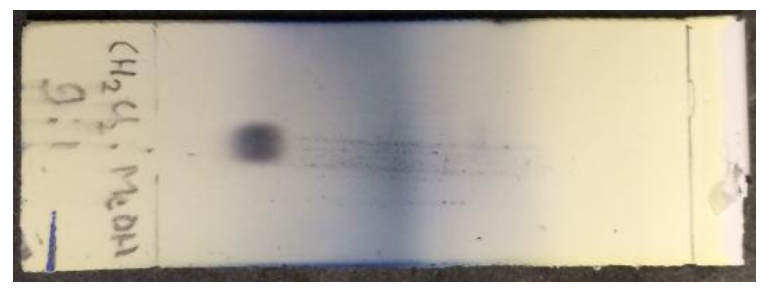


Comparison to the reported Trewianin glycoside ${ }^{1} H \mathrm{NMR}\left(700 \mathrm{MHz}, D_{5}\right.$-Pyridine):

\begin{tabular}{|c|c|c|}
\hline Carbon \# & $\begin{array}{l}\text { This work( Trewianin Aglycone) } \\
\delta_{\mathrm{H}} \text { (Multiplicity, } \mathrm{J} \text { in } \mathrm{Hz}, \text { No. of } \mathrm{H} \text { ) }\end{array}$ & $\begin{array}{c}\text { Seidel et al.(Trewianin) } \\
\delta_{\mathrm{H}} \text { (Multiplicity, } \mathrm{J} \text { in } \mathrm{Hz}, \mathrm{No} \text {. of } \mathrm{H} \text { ) }\end{array}$ \\
\hline 1 & $\begin{array}{c}1.42-1.30 \text { (ovrlp, } 1 \mathrm{H}) \\
3.53(\mathrm{dt}, J=13.9,3.4,1 \mathrm{H})\end{array}$ & $\begin{array}{l}1.27(\mathrm{td}, J=14.0,3.5,1 \mathrm{H}) \\
3.48(\mathrm{td}, J=14.0,3.5,1 \mathrm{H})\end{array}$ \\
\hline 2 & $\begin{array}{c}1.42-1.30(\text { ovrlp, } 1 \mathrm{H}) \\
2.25-2.20(\mathrm{~m}, 1 \mathrm{H})\end{array}$ & $\begin{array}{l}1.96 \mathrm{~m} \\
2.15 \mathrm{~m}\end{array}$ \\
\hline 3 & $\begin{array}{c}4.10-4.00(\mathrm{~m}, 1 \mathrm{H}) \\
6.02(\mathrm{~d}, J=4.2,1 \mathrm{H}, \mathrm{C} 3-\mathrm{OH})\end{array}$ & $3.92(\mathrm{dddd}, J=5.1,5.5,10.5,11.0,1 \mathrm{H})$ \\
\hline 4 & $\begin{array}{l}1.92-1.84 \text { (ovrlp, } 1 \mathrm{H}) \\
2.04-1.94 \text { (ovrlp, } 1 \mathrm{H})\end{array}$ & $\begin{array}{l}1.53(\mathrm{~m}, 1 \mathrm{H}) \\
1.79(\mathrm{~m}, 1 \mathrm{H})\end{array}$ \\
\hline 5 & $1.42-1.30$ (ovrlp, $1 \mathrm{H})$ & $1.20(\mathrm{~m}, 1 \mathrm{H})$ \\
\hline 6 & $1.42-1.30$ (ovrlp, $2 \mathrm{H}$ ) & $\begin{array}{l}1.22(\mathrm{~m}, 1 \mathrm{H}) \\
1.16(\mathrm{~m}, 1 \mathrm{H})\end{array}$ \\
\hline 7 & $\begin{array}{c}2.11 \text { (ovrlp, 1H) } \\
2.45(\mathrm{~m}, 1 \mathrm{H})\end{array}$ & $\begin{array}{l}1.18(\mathrm{~m}, 1 \mathrm{H}) \\
2.42(\mathrm{~m}, 1 \mathrm{H})\end{array}$ \\
\hline 8 & $2.17(\mathrm{td}, J=11.7,3.8,1 \mathrm{H})$ & $2.11(\mathrm{~m}, 1 \mathrm{H})$ \\
\hline 9 & $1.45(t, J=10.7,1 \mathrm{H})$ & $1.38(t, J=10.6,1 \mathrm{H})$ \\
\hline 10 & & \\
\hline 11 & $\begin{array}{c}4.65-4.54(\mathrm{~m}, 1 \mathrm{H}) \\
5.52-5.40 \text { (ovrlp, } 1 \mathrm{H}, \mathrm{C} 11-\mathrm{OH})\end{array}$ & $4.54(\mathrm{dt}, J=3.5,10.6,1 \mathrm{H})$ \\
\hline 12 & $\begin{array}{l}1.92-1.84 \text { (ovrlp, } 1 \mathrm{H}) \\
2.04-1.94 \text { (ovrlp, } 1 \mathrm{H})\end{array}$ & $\begin{array}{l}1.84(\mathrm{~m}, 1 \mathrm{H}) \\
1.98(\mathrm{~m}, 1 \mathrm{H})\end{array}$ \\
\hline 13 & & \\
\hline 14 & $5.52-5.40$ (ovrlp, $1 \mathrm{H}, \mathrm{C} 14-\mathrm{OH}$ ) & \\
\hline 15 & $\begin{array}{c}2.04-1.94 \text { (ovrlp, } 1 \mathrm{H}) \\
2.30(\mathrm{dt}, J=12.2,9.5,1 \mathrm{H})\end{array}$ & $\begin{array}{l}1.96(\mathrm{~m}, 1 \mathrm{H}) \\
2.27(\mathrm{~m}, 1 \mathrm{H})\end{array}$ \\
\hline 16 & $\begin{array}{l}2.11-2.08 \text { (ovrlp, } 1 \mathrm{H}) \\
2.04-1.94 \text { (ovrlp, } 1 \mathrm{H})\end{array}$ & $\begin{array}{l}1.95(\mathrm{~m}, 1 \mathrm{H}) \\
2.11(\mathrm{~m}, 1 \mathrm{H})\end{array}$ \\
\hline 17 & $2.94(\mathrm{dd}, J=9.4,5.2,1 \mathrm{H})$ & $2.89(\mathrm{dd}, J=4.8,9.2,1 \mathrm{H})$ \\
\hline 18 & $1.16(\mathrm{~s}, 3 \mathrm{H})$ & $1.12(\mathrm{~s}, 3 \mathrm{H})$ \\
\hline 19 & $\begin{array}{c}4.30(\mathrm{dd}, J=11.2,4.3,1 \mathrm{H}) \\
4.24(\mathrm{dd}, J=11.2,3.4,1 \mathrm{H}) \\
6.28(\mathrm{t}, J=4.0 \mathrm{~Hz}, 1 \mathrm{H}, \mathrm{C} 19-\mathrm{OH})\end{array}$ & $4.16(s)$ \\
\hline 20 & & \\
\hline 21 & $\begin{array}{l}5.30(\mathrm{dd}, J=18.0,1.6,1 \mathrm{H}) \\
5.04(\mathrm{dd}, J=18.0,1.5,1 \mathrm{H})\end{array}$ & $\begin{array}{l}5.01(\mathrm{dd}, J=1.7,18.0,1 \mathrm{H}) \\
5.28(\mathrm{dd}, J=1.7,18.0,1 \mathrm{H})\end{array}$ \\
\hline 22 & $6.12(\mathrm{~s}, 1 \mathrm{H})$ & $6.09(\mathrm{br} \mathrm{s}, 1 \mathrm{H})$ \\
\hline
\end{tabular}


Comparison of synthetic Trewianin aglycone (3) to Natural Trewianin:

\begin{tabular}{|c|c|c|c|}
\hline Carbon \# & Synthetic Trewianin Aglycone & Natural Trewianin $^{3}$ & $\boldsymbol{\Delta}(\boldsymbol{\delta})$ \\
\hline $\mathbf{1}$ & 35.8 & 35.3 & -0.5 \\
\hline $\mathbf{2}$ & 33.2 & 30.3 & -2.9 \\
\hline $\mathbf{3}$ & 70.4 & 75.6 & 5.2 \\
\hline $\mathbf{4}$ & 40.3 & 35.5 & -4.8 \\
\hline $\mathbf{5}$ & 45.6 & 45 & -0.6 \\
\hline $\mathbf{6}$ & 29.5 & 29.1 & -0.4 \\
\hline $\mathbf{7}$ & 28.3 & 28.1 & -0.2 \\
\hline $\mathbf{8}$ & 42.2 & 41.9 & -0.3 \\
\hline $\mathbf{9}$ & 55.5 & 55.1 & -0.4 \\
\hline $\mathbf{1 0}$ & 41.6 & 41.4 & -0.2 \\
\hline $\mathbf{1 1}$ & 70.2 & 69.9 & -0.3 \\
\hline $\mathbf{1 2}$ & 50.0 & 49.7 & -0.3 \\
\hline $\mathbf{1 3}$ & 50.6 & 50.4 & -0.2 \\
\hline $\mathbf{1 4}$ & 84.7 & 84.5 & -0.2 \\
\hline $\mathbf{1 5}$ & 33.6 & 33.3 & -0.3 \\
\hline $\mathbf{1 6}$ & 27.5 & 27.3 & -0.3 \\
\hline $\mathbf{1 7}$ & 51.4 & 51.1 & -0.3 \\
\hline $\mathbf{1 8}$ & 18.0 & 17.7 & -0.3 \\
\hline $\mathbf{1 9}$ & 59.5 & 59.1 & -0.4 \\
\hline $\mathbf{2 0}$ & 174.5 & 174.5 & 0.0 \\
\hline $\mathbf{2 1}$ & 73.8 & 73.7 & -0.1 \\
\hline $\mathbf{2 2}$ & 117.8 & 117.6 & -0.2 \\
\hline $\mathbf{2 3}$ & 175.8 & 175.6 & 0.2 \\
\hline
\end{tabular}
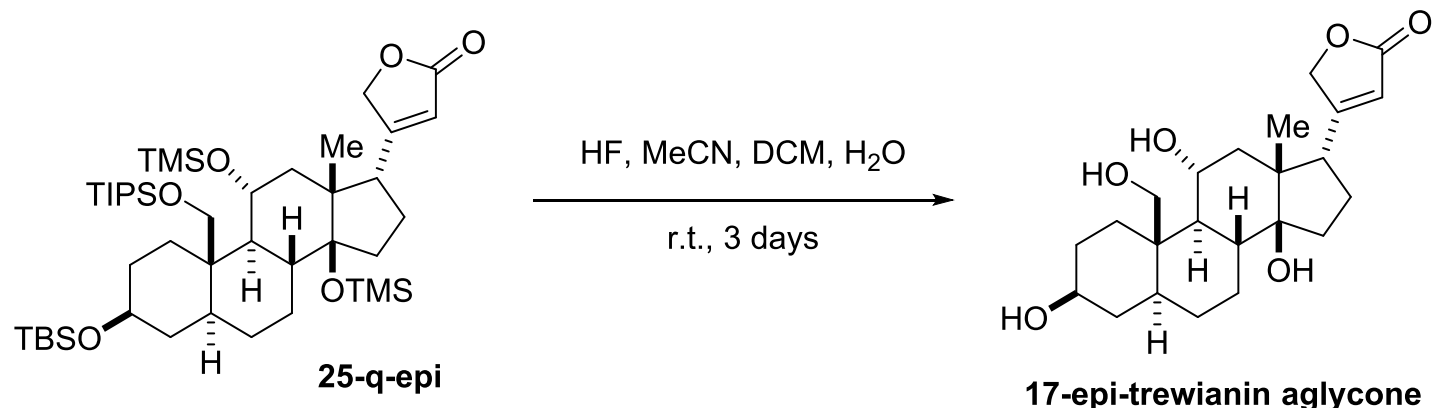

17-epi-trewianin aglycone

17-epi-trewianin aglycone. Steroid 25-q-epi $(5.0 \mathrm{mg}, 0.00609 \mathrm{mmol})$ was solvated in a 1:1 mixture of $\mathrm{MeCN}: \mathrm{CH}_{2} \mathrm{Cl}_{2}(600 \mu \mathrm{L})$. A solution of 18:3:1 MeCN: $\mathrm{H}_{2} \mathrm{O}: \mathrm{HF}$ was prepared and $600 \mu \mathrm{L}$ of this stock solution was added to the reaction mixture. The solution was allowed to stir at room temperature for $60 \mathrm{~h}$. Then, $1 \mathrm{~mL}$ of methoxytrimethyl silane was added and the solution was stirred for 30 minutes. Then, the crude material was concentrated in vacuo. The resulting residue was directly purified by flash column 
chromatography with $0 \% \rightarrow 10 \% \mathrm{MeOH}$ in $\mathrm{CH}_{2} \mathrm{Cl}_{2}$ to afford 17-epi-trewianin aglycone $(2.2 \mathrm{mg}, 0.0054$ $\mathrm{mmol})$ in $89 \%$ yield as a white solid. ${ }^{1} \mathrm{H}$ NMR $\left(700 \mathrm{MHz}, \mathrm{D}_{5}\right.$-Pyridine): $\delta 6.32(\mathrm{~s}, 1 \mathrm{H},-\mathrm{OH}), 6.10(\mathrm{~s}, 1 \mathrm{H}$, $\mathrm{H} 22), 6.04(\mathrm{~s}, 1 \mathrm{H},-\mathrm{OH}), 5.47(\mathrm{~s}, 1 \mathrm{H},-\mathrm{OH}), 5.42(\mathrm{~s}, 1 \mathrm{H},-\mathrm{OH}), 4.94(\mathrm{~d}, J=17.8 \mathrm{~Hz}, 1 \mathrm{H}, \mathrm{H} 21 \mathrm{a}), 4.81(\mathrm{~d}, J=$ $17.6 \mathrm{~Hz}, 1 \mathrm{H}, \mathrm{H} 21 \mathrm{~b}), 4.58-4.45(\mathrm{~m}, 1 \mathrm{H}, \mathrm{H} 11), 4.32(\mathrm{~d}, J=9.9 \mathrm{~Hz}, 1 \mathrm{H}, \mathrm{H} 19 \mathrm{a}), 4.28(\mathrm{~d}, J=11.0 \mathrm{~Hz}, 1 \mathrm{H}$, $\mathrm{H} 19 \mathrm{~b}), 4.07(\mathrm{~m}, 1 \mathrm{H}, \mathrm{H} 3), 3.55(\mathrm{~d}, J=14.0 \mathrm{~Hz}, 1 \mathrm{H}, \mathrm{H} 1 \mathrm{a}), 3.40$ (t, J = 9.6 Hz, 1H, H17), 2.51 (d, J = $11.1 \mathrm{~Hz}$, $1 \mathrm{H}, \mathrm{H} 7 \mathrm{a}$ ), 2.31 (td, $J=13.2,4.6 \mathrm{~Hz}, 1 \mathrm{H}, \mathrm{H} 15 \mathrm{a}$ ), 2.23 (ovrlp, 2H, H2a, 8), 2.16 - 2.04 (ovrlp, 2H, H2b, 16a), 2.01 (m, 1H, H4a), 1.96 - 1.81 (ovrlp, 3H, H4b, 15b, 16b), 1.67 (m, 2H, H12a, 12b), 1.46 - 1.18 (ovrlp, 9H, $\mathrm{H} 1 \mathrm{~b}, 5,6 \mathrm{a}, 6 \mathrm{~b}, 9,18) .{ }^{13} \mathrm{C}$ NMR (175 MHz, $\mathrm{D}_{5}$-pyridine) $\delta 174.1(\mathrm{C}-23), 172.5(\mathrm{C}-20), 116.7(\mathrm{C}-22), 85.2(\mathrm{C}-$ 14), 74.1(C-21), 70.4(C-3), 70.2(C-11), 59.5(C-19), 55.7(C-9), 50.1(C-13), 49.4(C-17), 45.6(C-5), 42.2(C-8), 41.6(C-10), 41.6(C-12), 40.3(C-4), 35.9(C-1), 33.1(C-2), 32.0(C-15), 29.5(C-6), 27.9(C-7), 25.0(C-16), 20.3(C-18). HRMS (ESI): calculated for $\mathrm{C}_{23} \mathrm{H}_{34} \mathrm{O}_{6} \mathrm{Na}^{+}[\mathrm{M}+\mathrm{Na}]^{+} 429.2248$, found 429.2251. IR (film, $\mathrm{cm}^{-1}$ ):

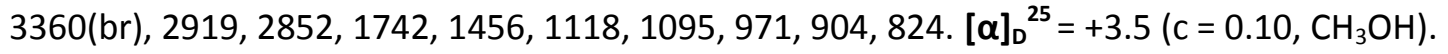

\section{Comparison of C17-epi-Trewianin aglycone to Natural Trewianin:}

\begin{tabular}{|c|c|c|c|}
\hline Carbon \# & C17-epi-Trewianin Aglycone & Natural Trewianin $^{3}$ & $\boldsymbol{\Delta}(\boldsymbol{\delta})$ \\
\hline $\mathbf{1}$ & 35.9 & 35.3 & -0.6 \\
\hline $\mathbf{2}$ & 33.1 & 30.3 & -2.8 \\
\hline $\mathbf{3}$ & 70.4 & 75.6 & 5.2 \\
\hline $\mathbf{4}$ & 40.3 & 35.5 & -4.8 \\
\hline $\mathbf{5}$ & 45.6 & 45 & -0.6 \\
\hline $\mathbf{6}$ & 29.5 & 29.1 & -0.4 \\
\hline $\mathbf{7}$ & 27.9 & 28.1 & 0.2 \\
\hline $\mathbf{8}$ & 42.2 & 41.9 & -0.3 \\
\hline $\mathbf{9}$ & 55.7 & 55.1 & -0.6 \\
\hline $\mathbf{1 0}$ & 41.6 & 41.4 & -0.2 \\
\hline $\mathbf{1 1}$ & 70.2 & 69.9 & -0.3 \\
\hline $\mathbf{1 2}$ & 41.6 & 49.7 & 8.1 \\
\hline $\mathbf{1 3}$ & 50.1 & 50.4 & 0.3 \\
\hline $\mathbf{1 4}$ & 85.2 & 84.5 & -0.7 \\
\hline $\mathbf{1 5}$ & 32.0 & 33.3 & 1.3 \\
\hline $\mathbf{1 6}$ & 25.0 & 27.3 & 2.3 \\
\hline $\mathbf{1 7}$ & 49.4 & 51.1 & 0.7 \\
\hline $\mathbf{1 8}$ & 20.3 & 17.7 & -2.6 \\
\hline $\mathbf{1 9}$ & 59.5 & 59.1 & -0.4 \\
\hline $\mathbf{2 0}$ & 172.5 & 174.5 & 2.0 \\
\hline $\mathbf{2 1}$ & 74.1 & 73.7 & -0.4 \\
\hline $\mathbf{2 2}$ & 116.7 & 117.6 & 0.9 \\
\hline $\mathbf{2 3}$ & 174.1 & 175.6 & 1.5 \\
\hline & & & \\
\hline
\end{tabular}


Unnatural C/D junction:
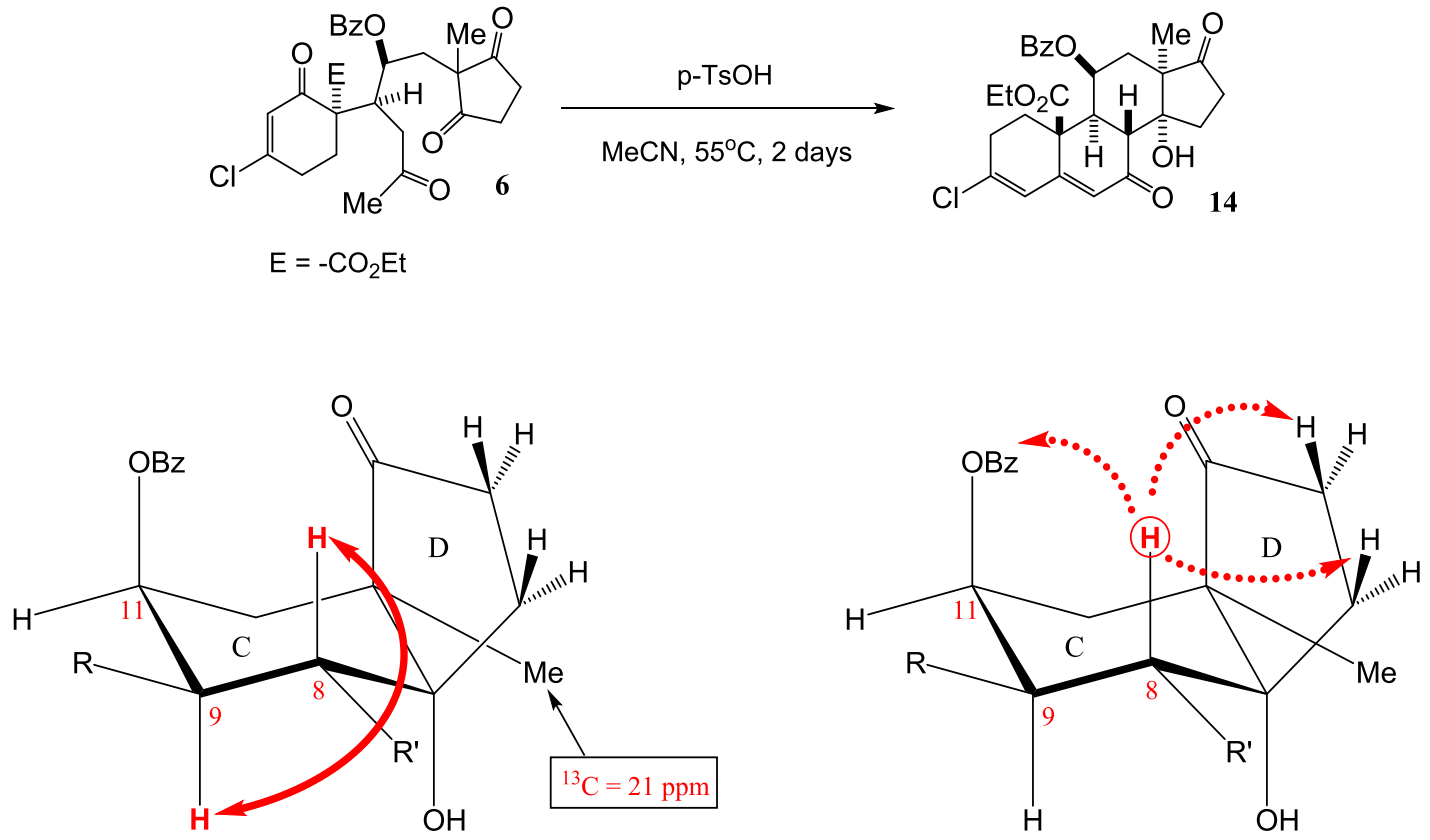

$J=13.2 \mathrm{~Hz}$

Transdiaxial

NOE correlations

Natural C/D junction:
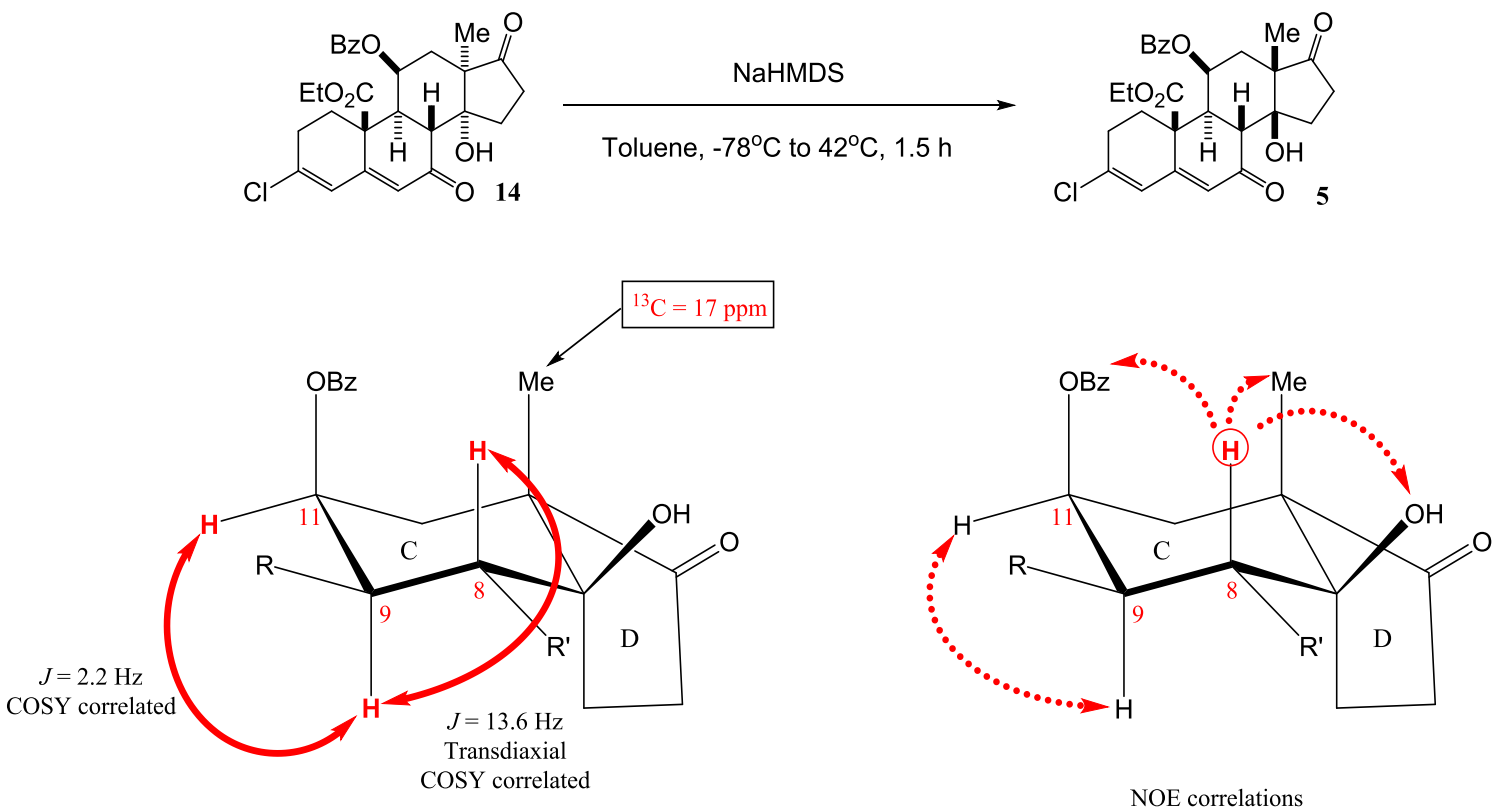
C18 Carbon shift: In addition to the more concrete structural proof, our research into the topic of steroidal C/D ring junctions revealed an interesting and strong correlation between the C18 carbon NMR shift and the ring junction stereochemistry. It became apparent that steroids of similar structure having a C/D-cis junction with $\beta$-oriented $-\mathrm{Me}$ and $\beta$-oriented $-\mathrm{OH}$ (natural junction) often produce a characteristic C18 shift of $15-17$ ppm. In contrast, a C/D-cis junction with $\alpha$-oriented $-\mathrm{Me}$ and $\alpha$ oriented $-\mathrm{OH}$ produce a $\mathrm{C} 18$ shift in the range of $20-21 \mathrm{ppm}^{4}$. This is a theme we have noticed in the literature and throughout our own work on the synthesis of cardenolides.

\section{A/B-cis reduction:}
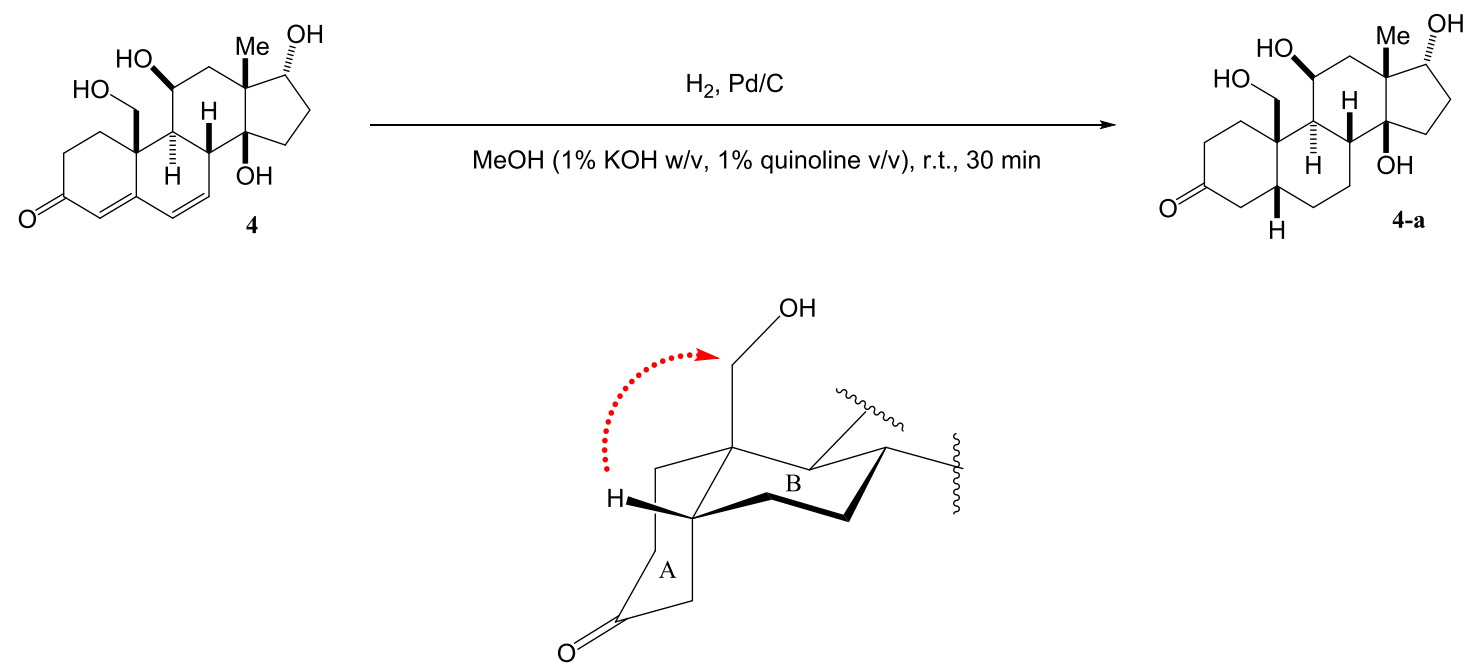

NOE correlations

\section{C3 reduction (Trewianin Aglycone):}

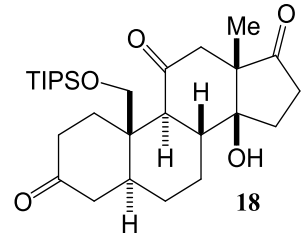

H

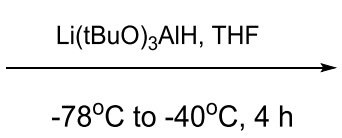

$-78^{\circ} \mathrm{C}$ to $-40^{\circ} \mathrm{C}, 4 \mathrm{~h}$

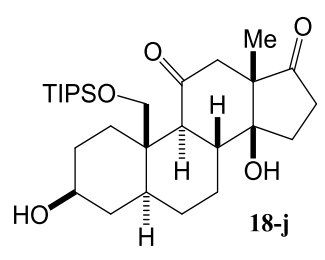

18-j

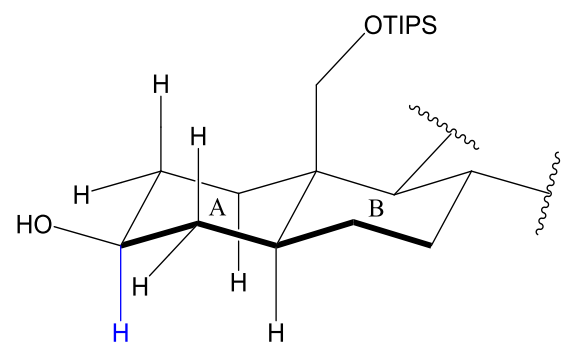

( tt, $J=10.5,5.2$ )

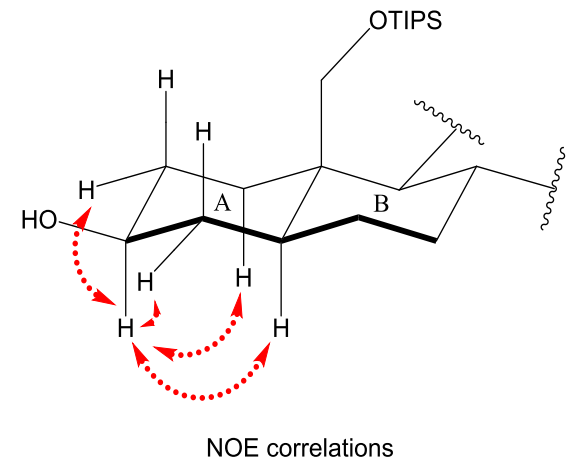




\section{C16-C17 Hydrogenation (Trewianin Aglycone)}

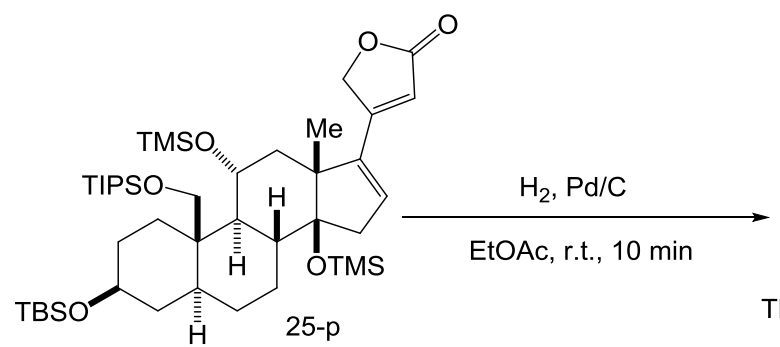

H $25-p$

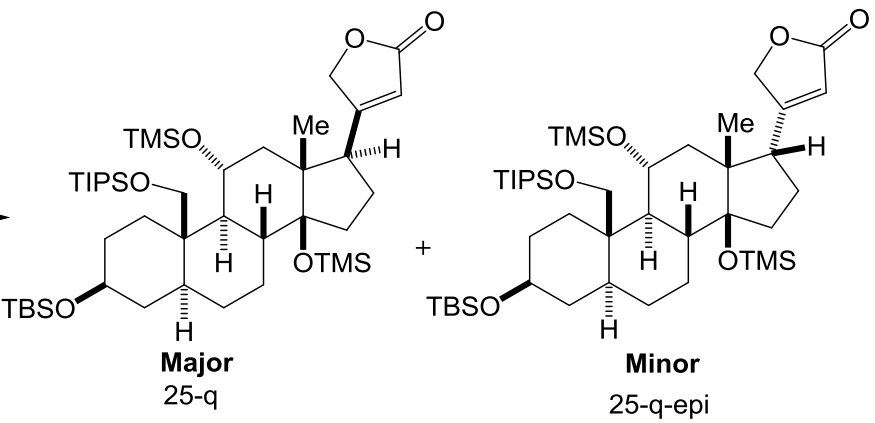

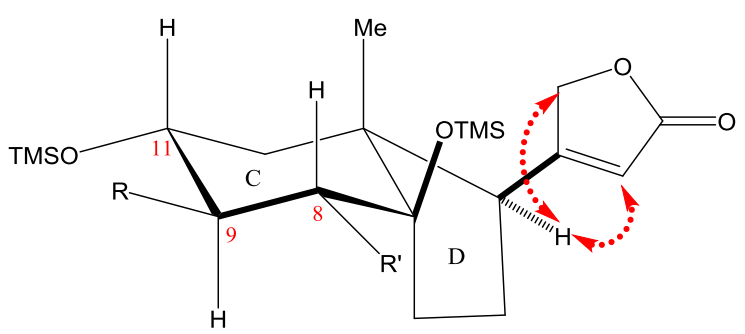

Major 25-q

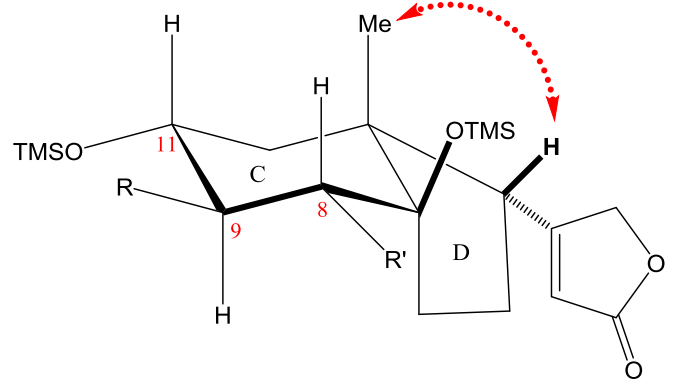

NOE correlations
Minor

25-q-epi

$\underline{\mathrm{X}-\mathrm{RAY} \text { analysis in model system: }}$<smiles>CCOC(=O)C1CCCCC1=O</smiles>

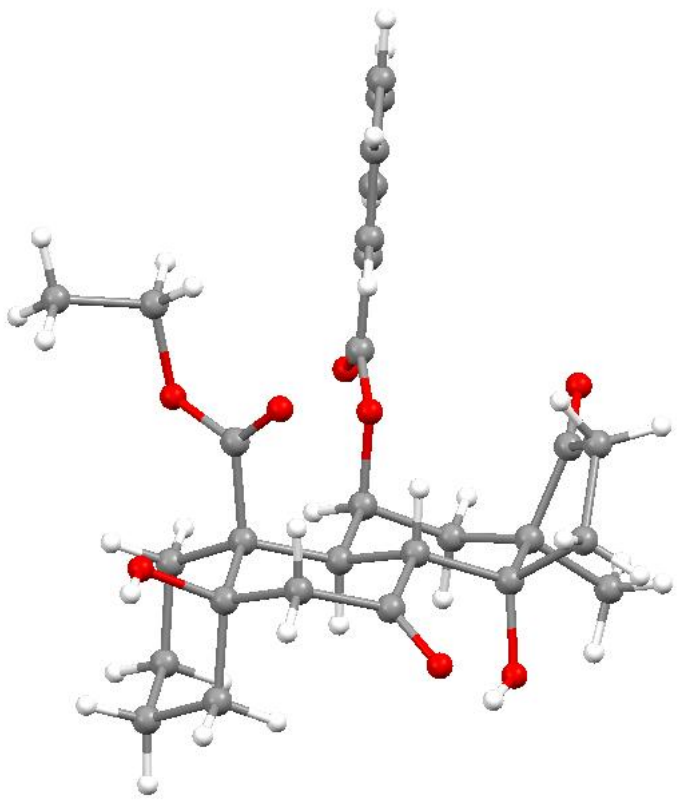

1) $\mathrm{Cu}(\mathrm{OTf})_{2}$, r.t., o/n

2) THF, DBU, reflux, o/n

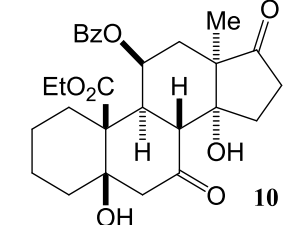

$(+/-)$ 


\section{Target Steroids:}

19-Hydroxysarmentogenin (2): The structure of 19-hydroxysarmentogenin is known in the literature and all data matches the known data precisely. The optical rotation indicates the desired, natural (+)enantiomer was synthesized.

Panogenin core (17): All NMR data are consistent with the observed stereochemistry, including the following relevant NOE correlations:

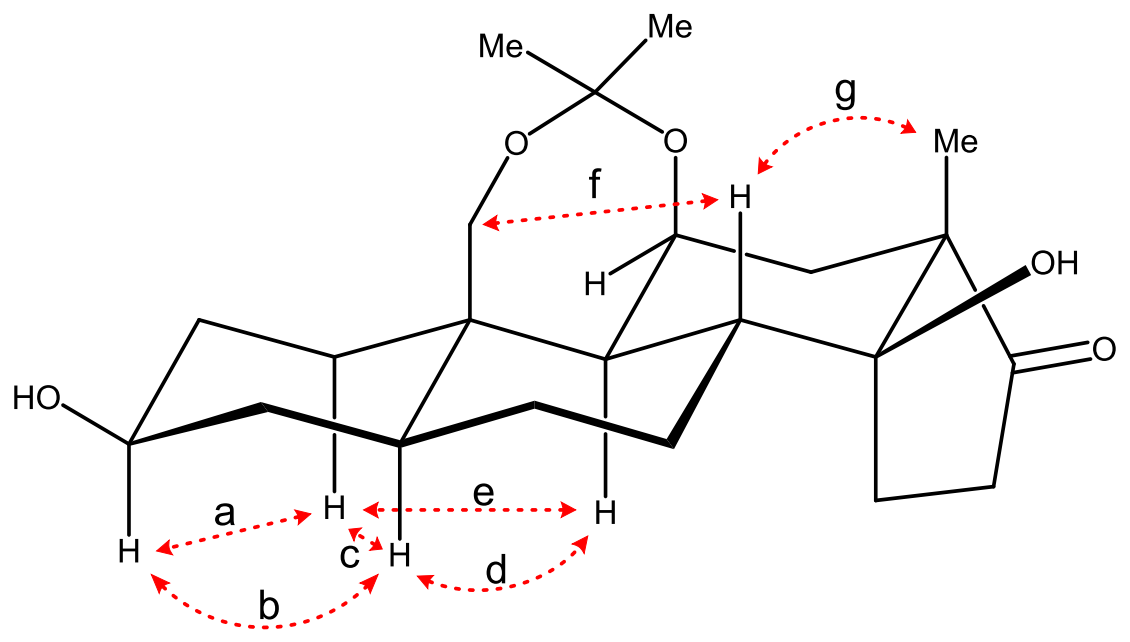

(letters reference to NOE spectra found on page S77)

Trewianin aglycone (3): As this structure is unknown in the literature, all structural data was compared to reported values for the natural glycoside to verify structural assignment and detailed structural characterization on the target compound and key intermediates was performed with NMR analysis. Observed NOESY correlations for Trewianin aglycone (3):

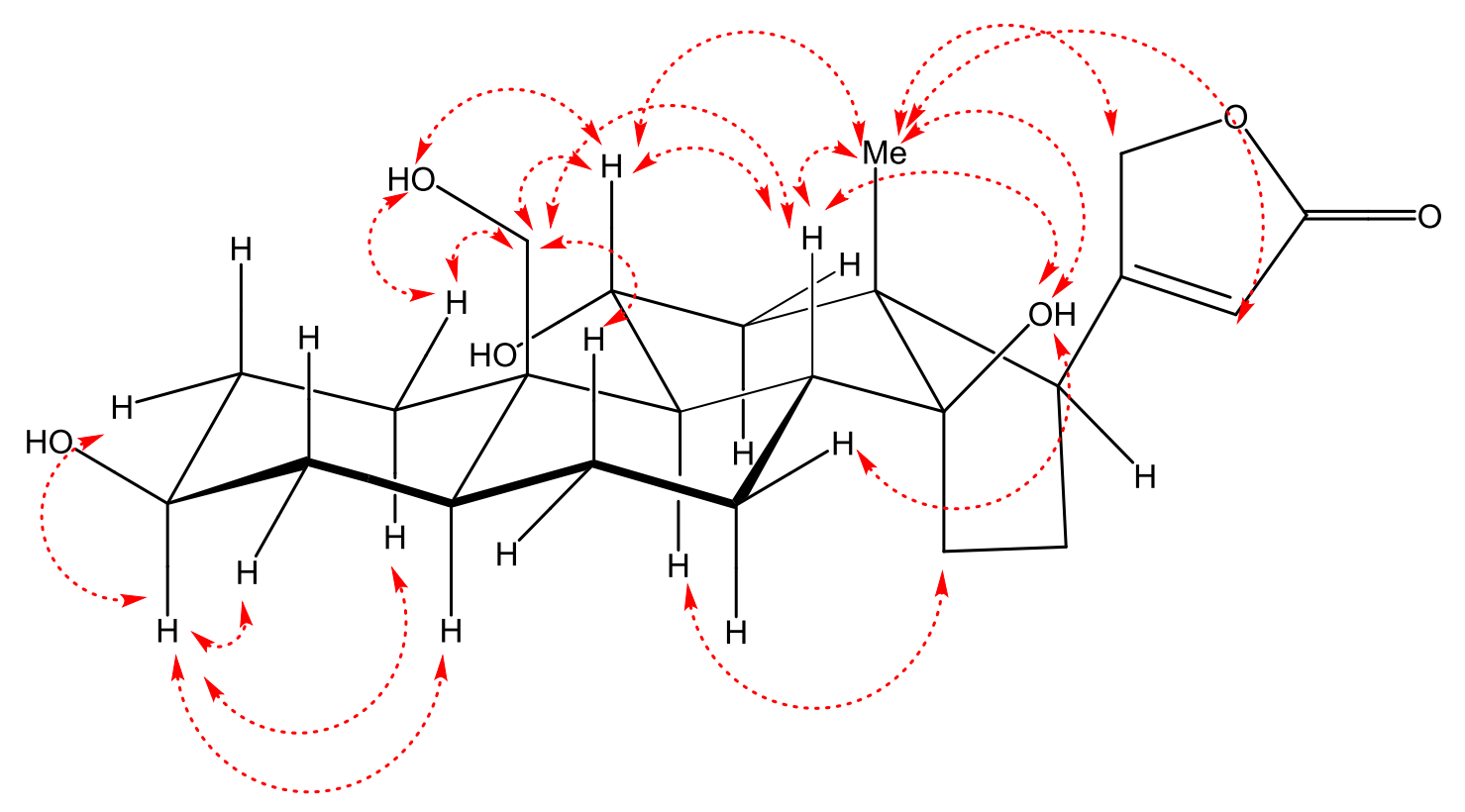


Structural evidence derived from ${ }^{13} \mathrm{C}$ NMR shift correlations:

A thorough collection and review of cardiac glycoside ${ }^{13} \mathrm{C}$ NMR shifts was published by Wolfgang Robien et al. ${ }^{4}$ Several strong correlations and patterns relating to specific ${ }^{13} \mathrm{C}$ shifts and stereochemical/structural features were noted. In particular, certain isomers often show characteristic carbon shifts which deviate significantly enough from its other isomers to constitute evidence of a steroids configuration. In addition to the characteristic C18 shifts mentioned earlier (page 29), the authors noted the following tell-tale shifts:

\begin{tabular}{|c|c|c|}
\hline Carbon No. & $\mathbf{5} \boldsymbol{\alpha}$ - derivatives & $\mathbf{5 \beta}$ - derivatives \\
\hline C5 & $44.7 \pm 0.4$ & $36.0 \pm 0.6$ \\
\hline C9 & $50.0 \pm 0.3$ & $35.5 \pm 0.4$ \\
\hline
\end{tabular}

Accordingly, our synthetic sample of Trewianin aglycone shows carbon shifts of:
C5: 45.62
C9: 55.45

These shifts are in accord with those noted for $5 \alpha$-derivatives.

And our sample of Panogenin core shows carbon shifts of:
C5: 43.02
C9: 51.05

Again, in agreement with known shifts for $5 \alpha$-derivatives.

For 19-hydroxysarmentogenin, the shifts are:
C5: 32.28
C9: 41.61

In better alignment with those expected for $5 \beta$-derivatives. 


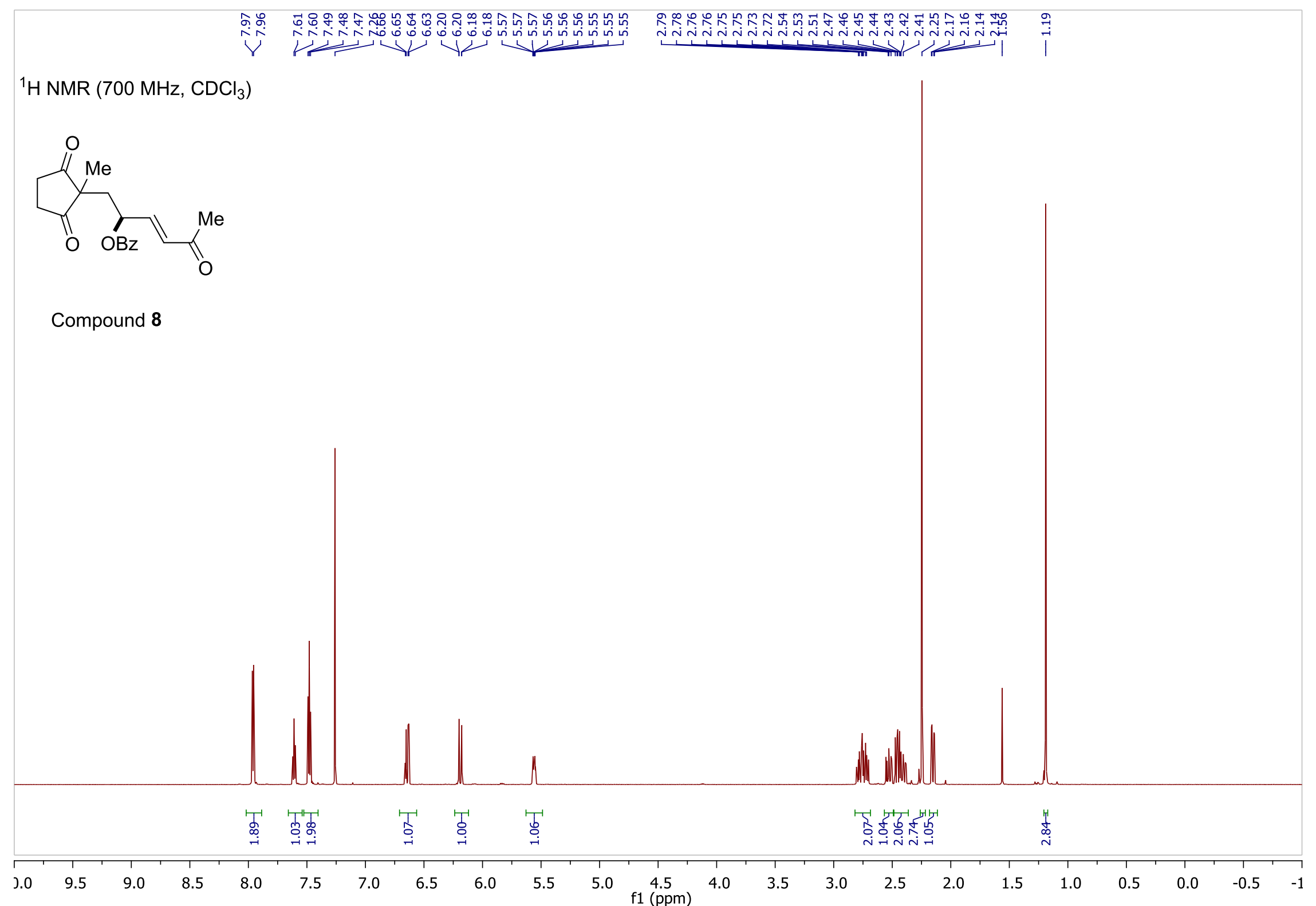




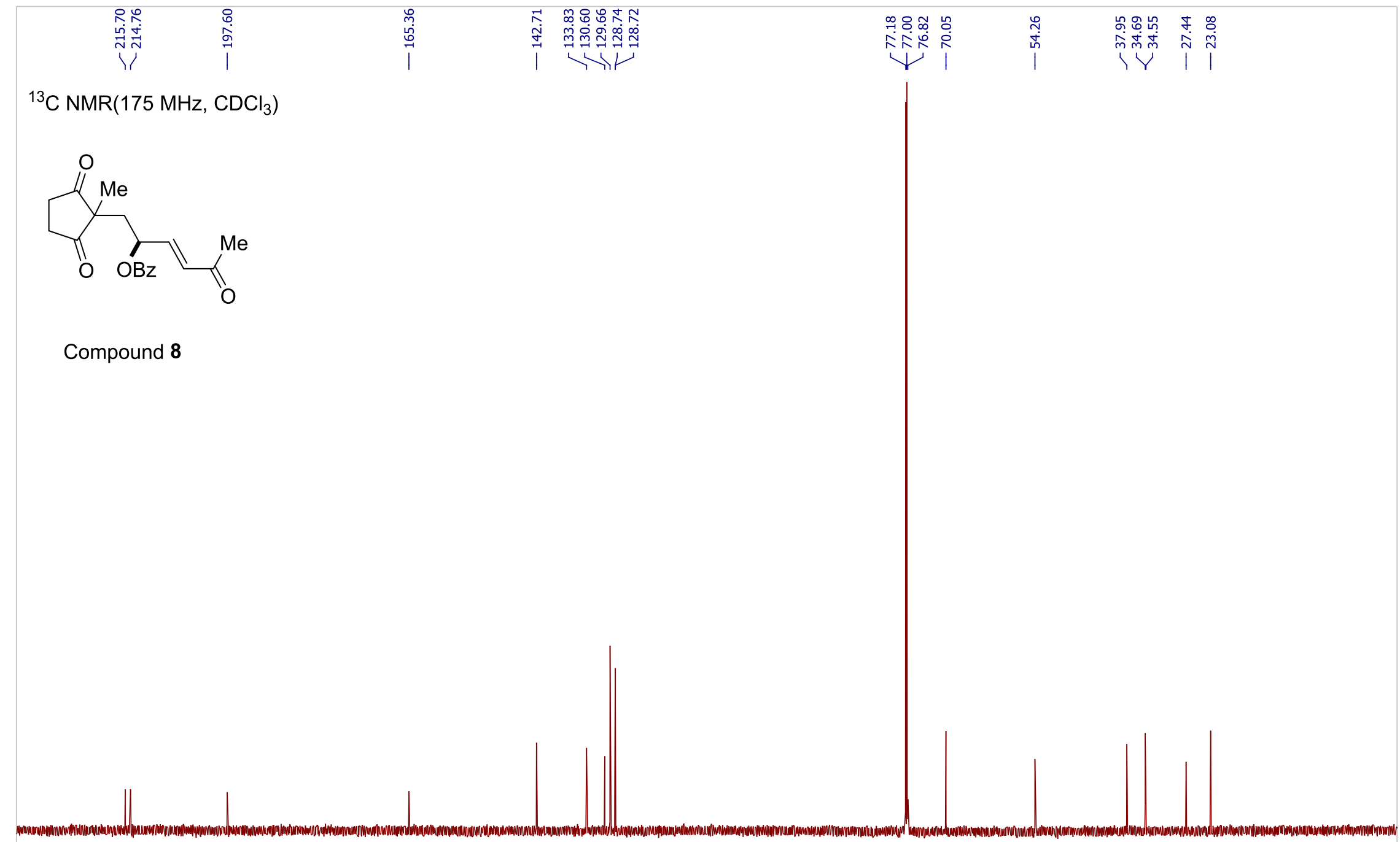


${ }^{1} \mathrm{H}$ NMR $\left(500 \mathrm{MHz}, \mathrm{CDCl}_{3}\right)$

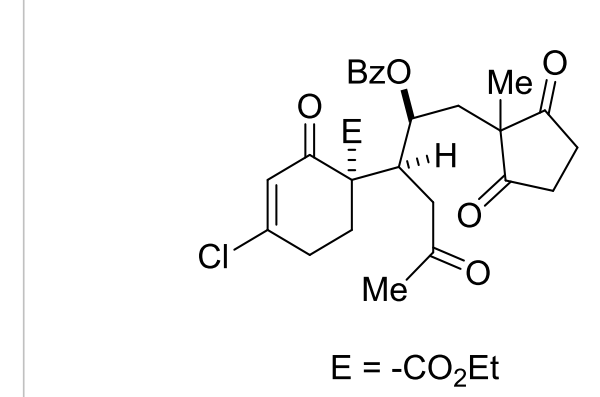

Compound 6

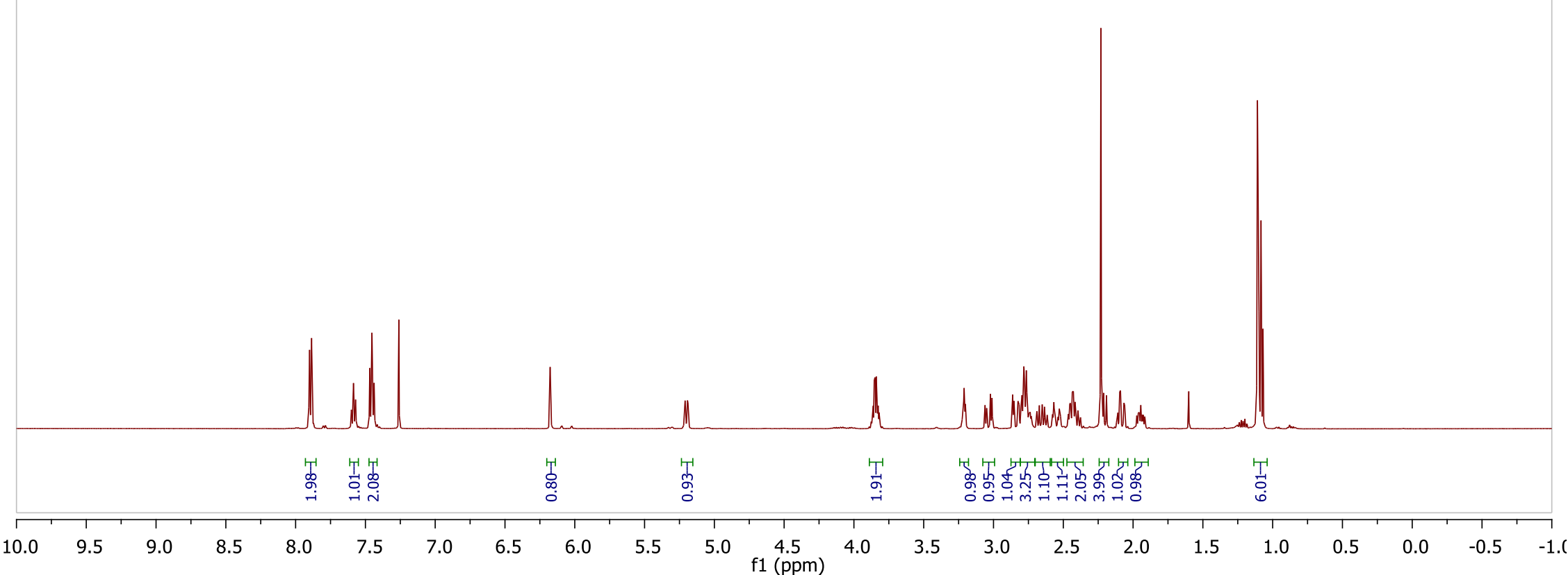




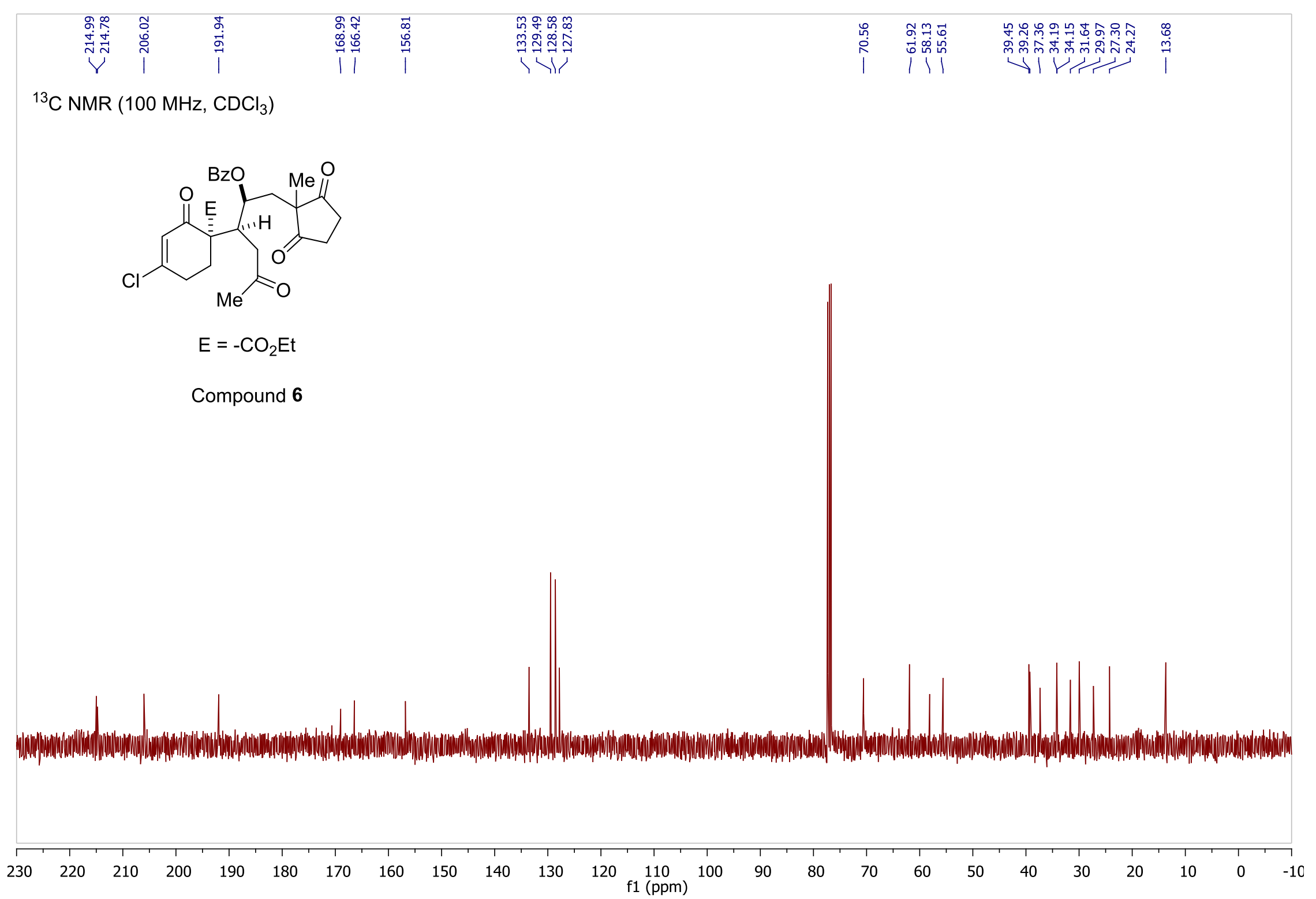




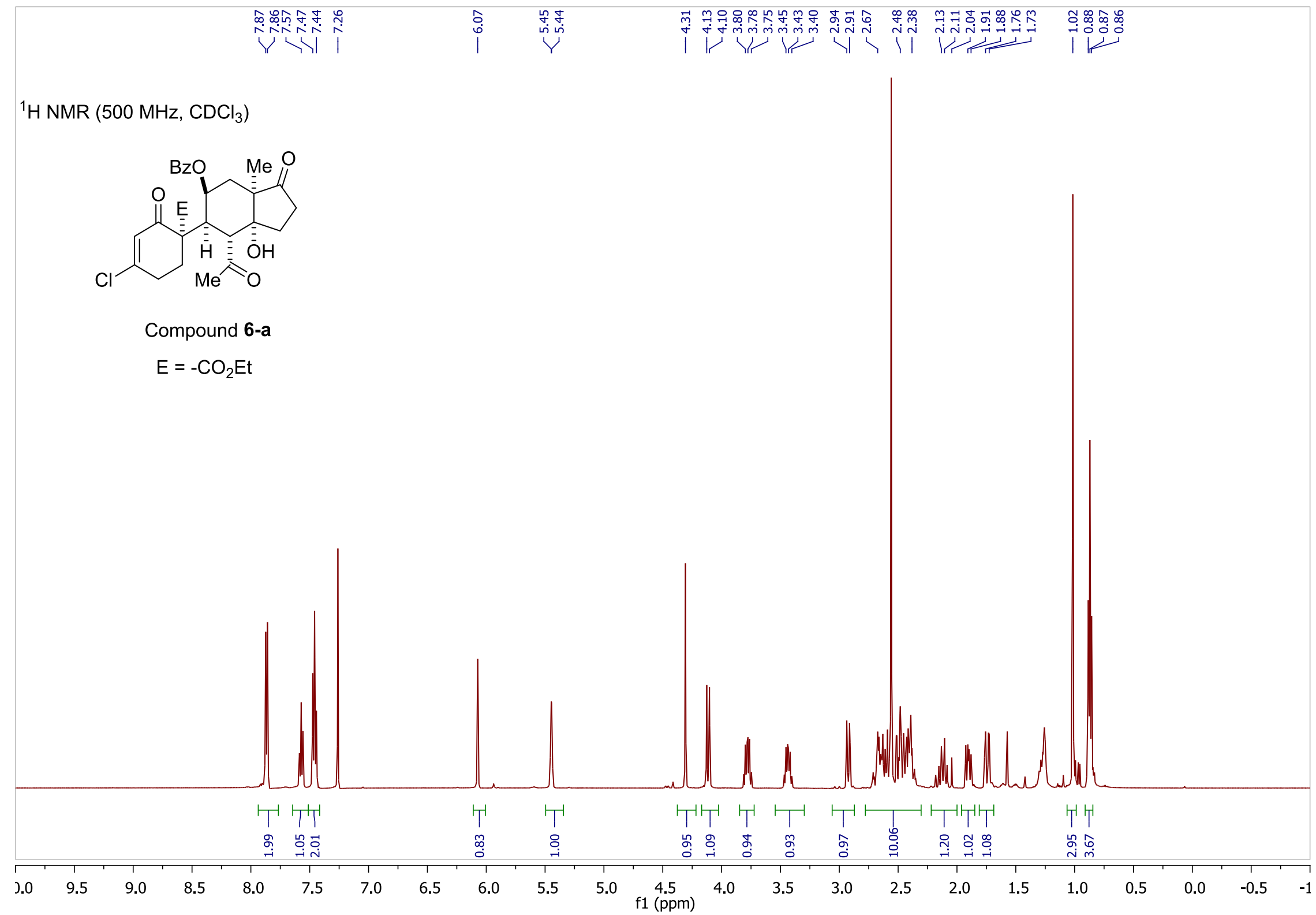




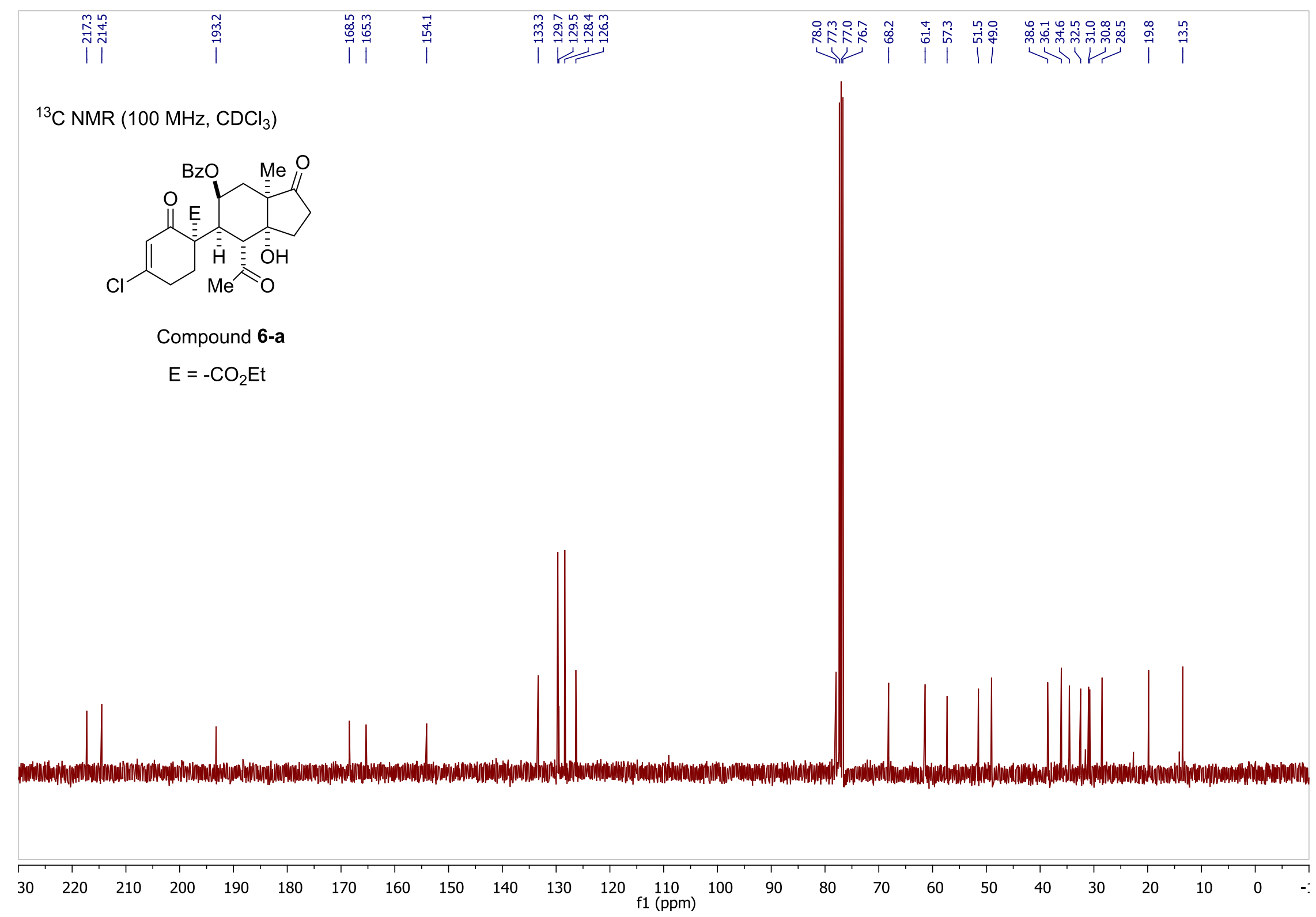




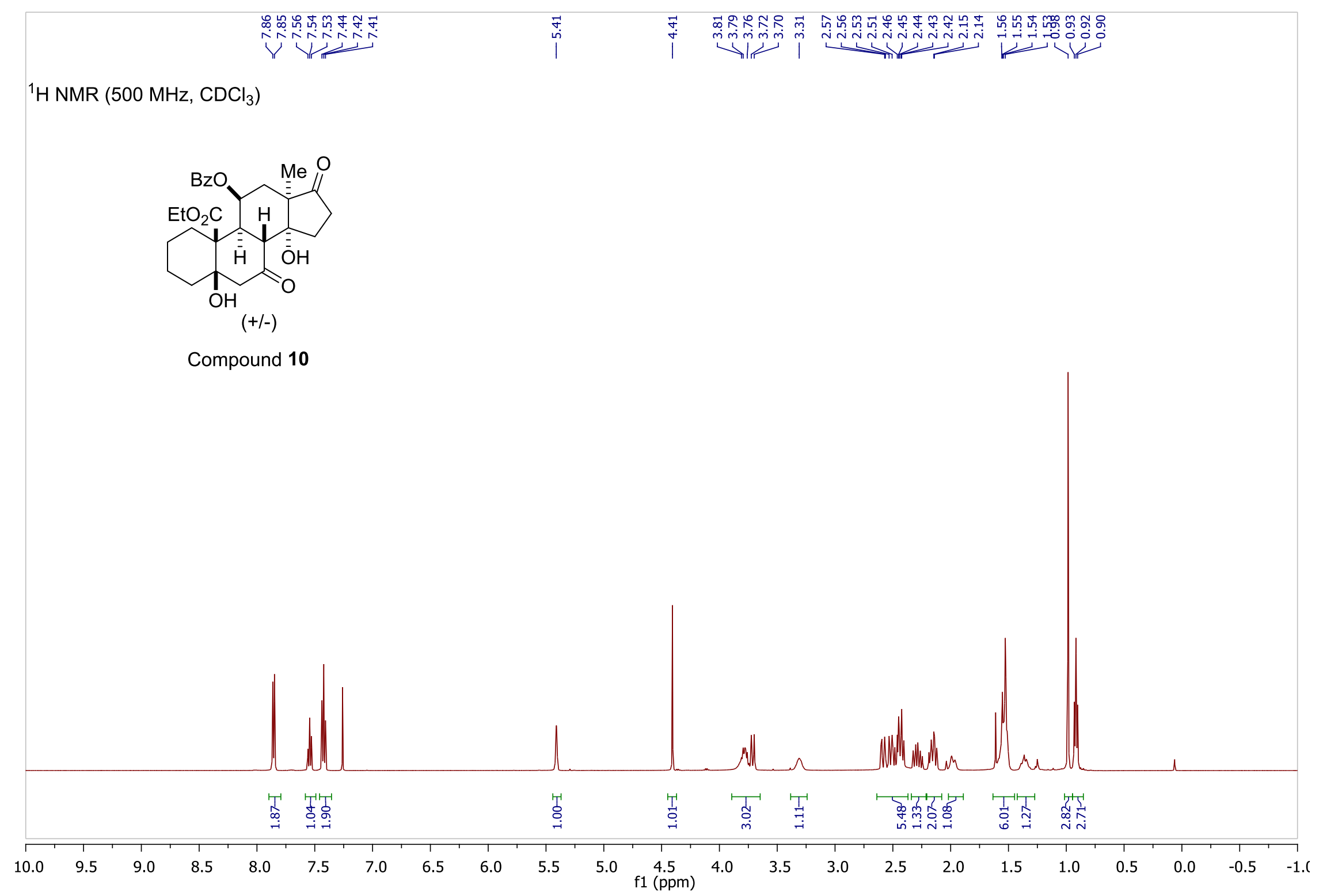



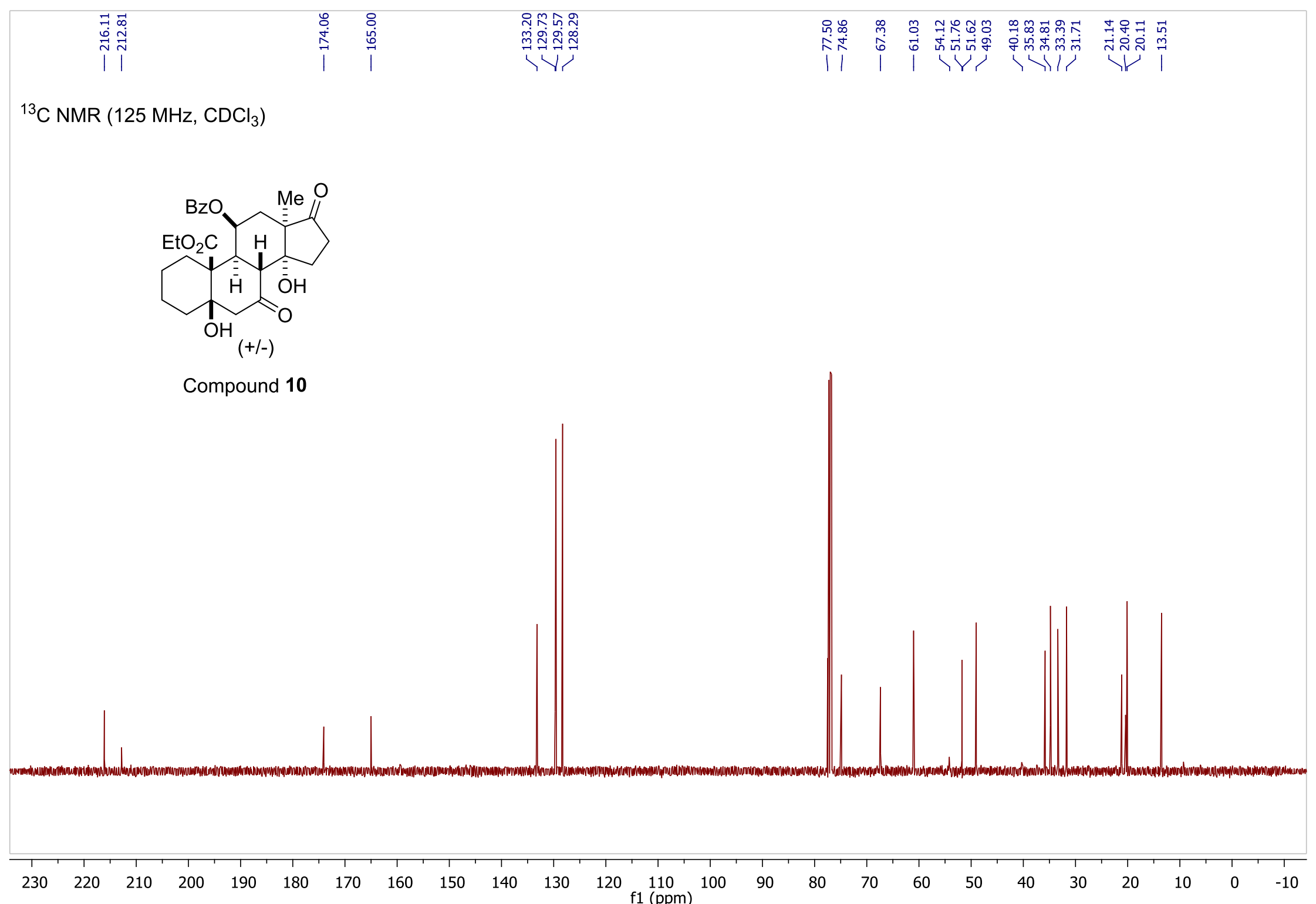


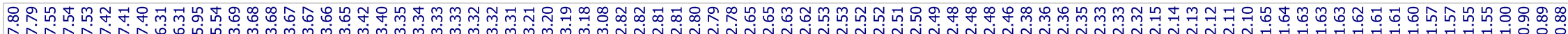

${ }^{1} \mathrm{H}$ NMR $\left(700 \mathrm{MHz}, \mathrm{CDCl}_{3}\right)$

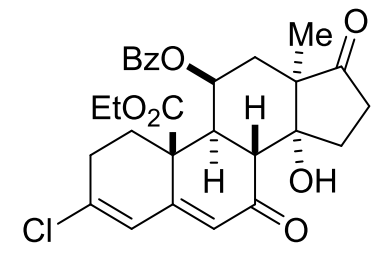

Compound 14

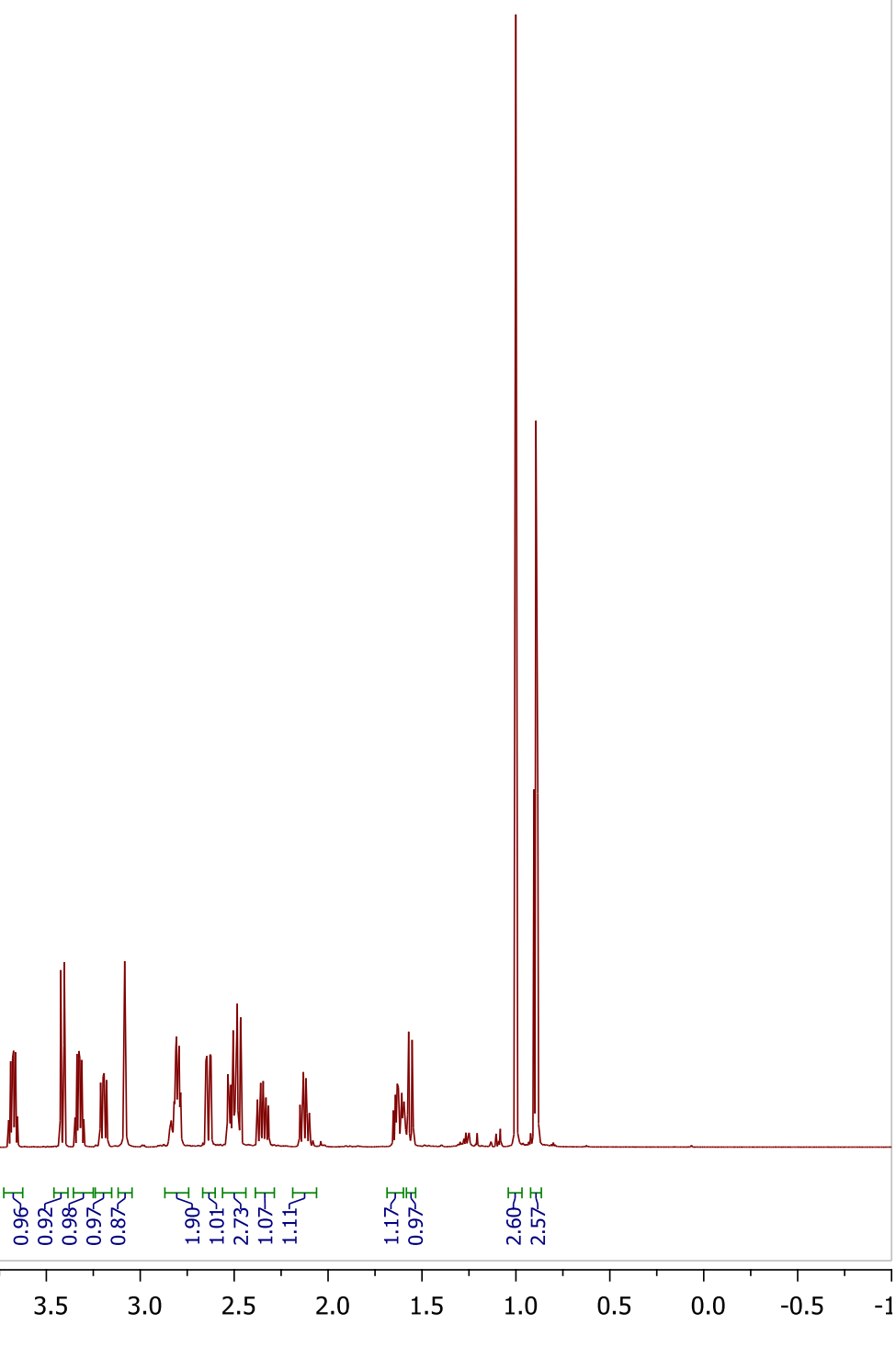



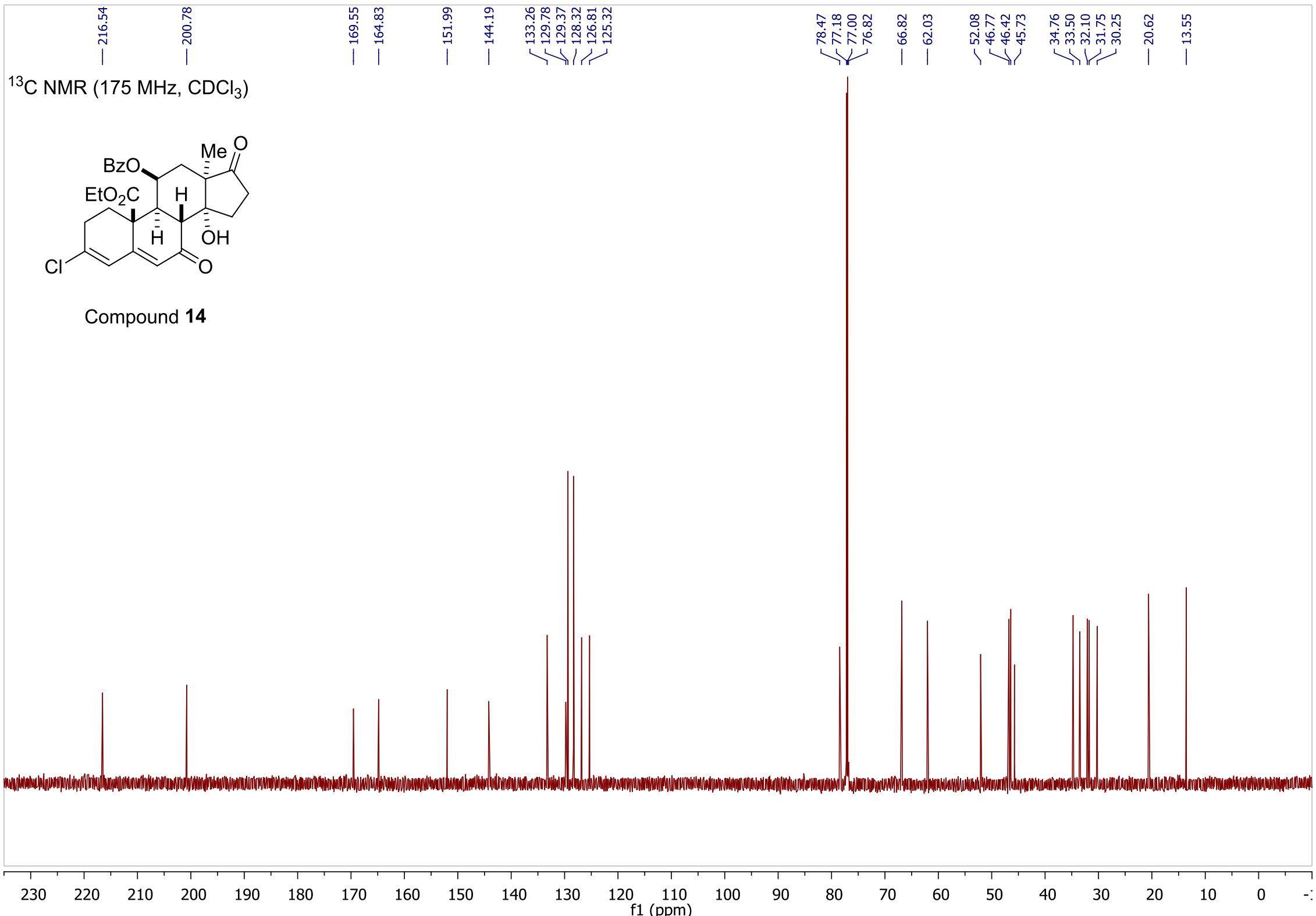
${ }^{1} \mathrm{H}$ NMR $\left(700 \mathrm{MHz}, \mathrm{CDCl}_{3}\right)$

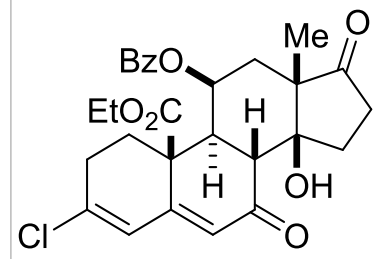

Compound 5

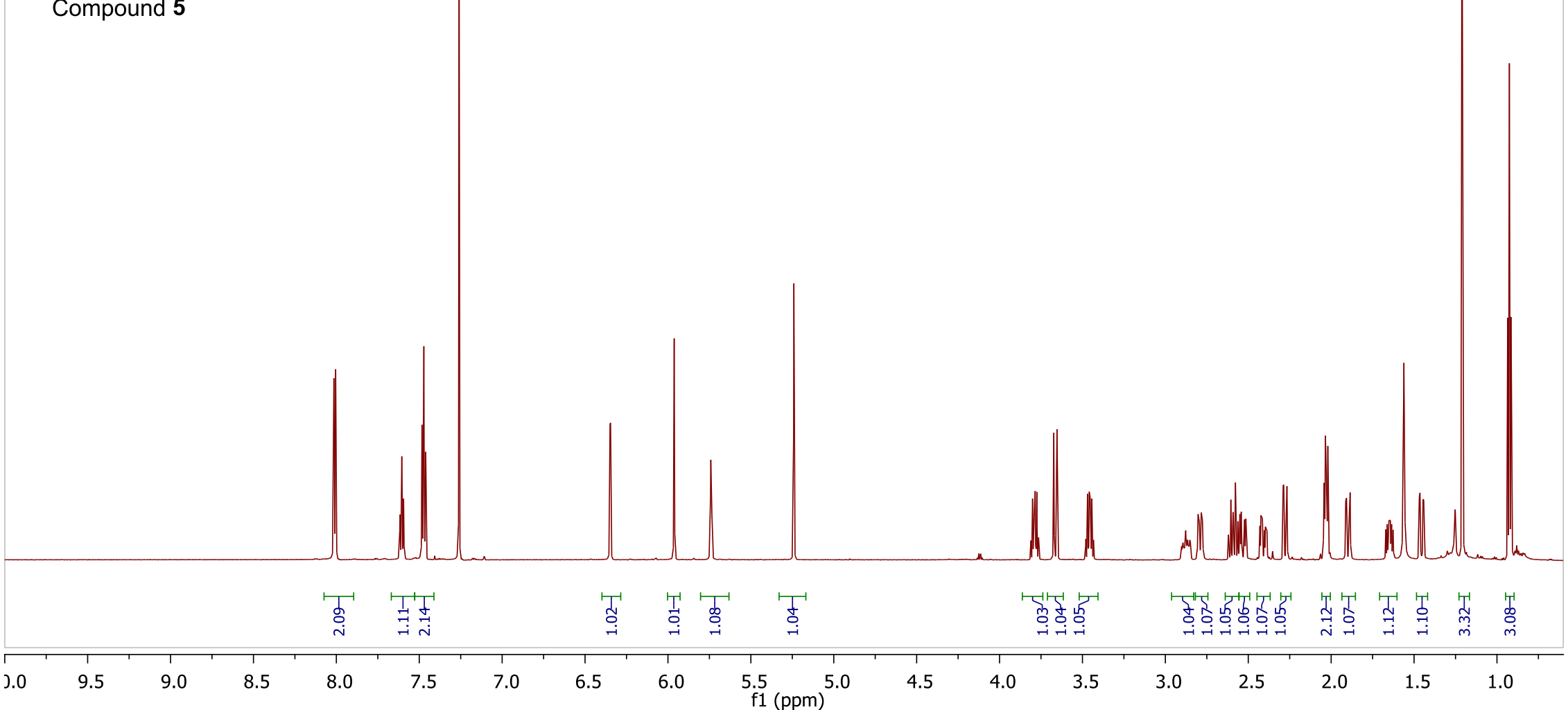




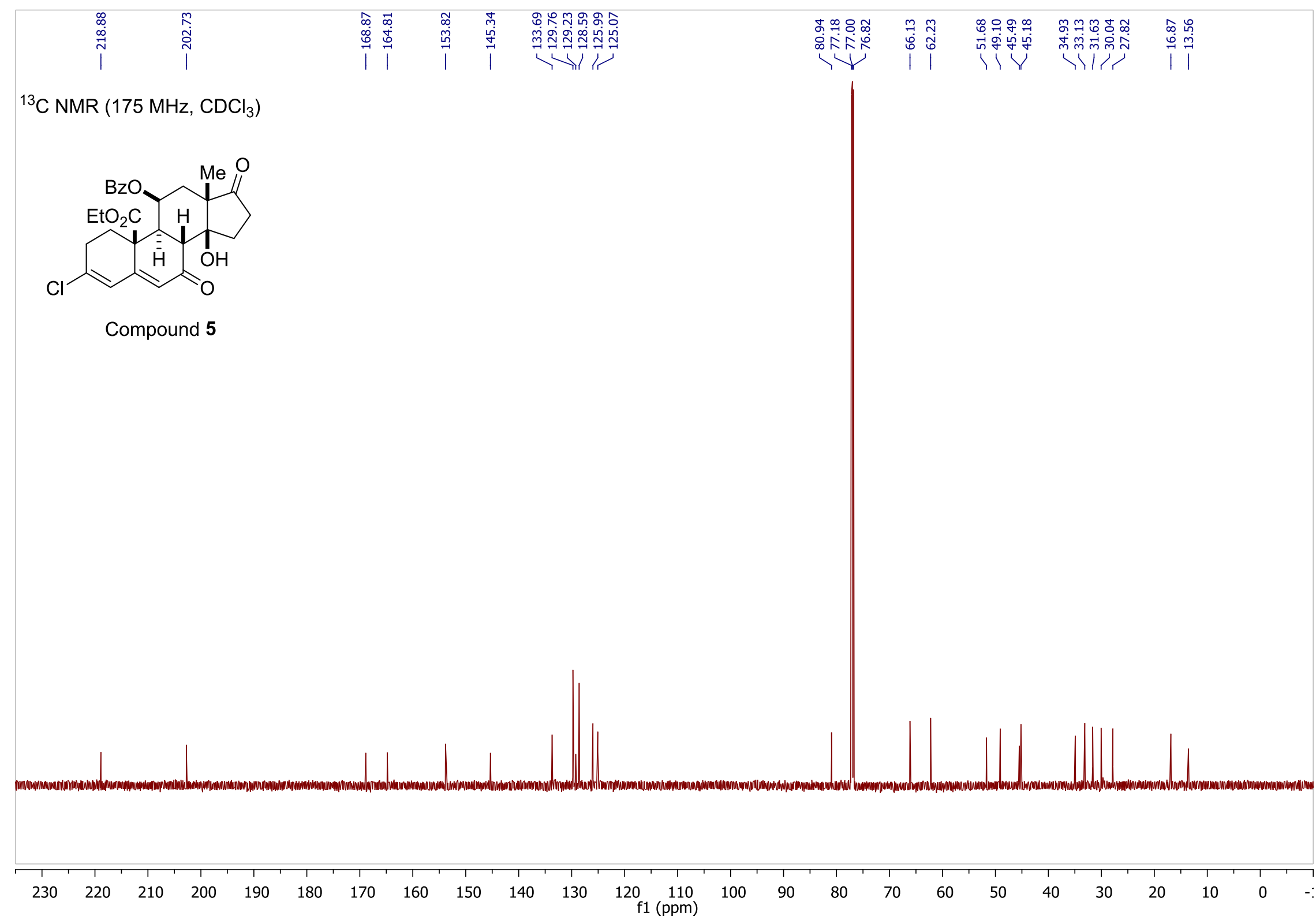




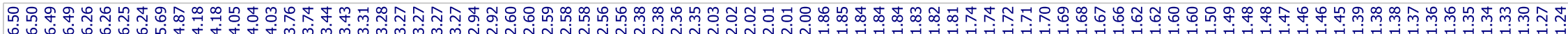

${ }^{1} \mathrm{H}$ NMR $\left(700 \mathrm{MHz}, \mathrm{CD}_{3} \mathrm{OD}\right)$

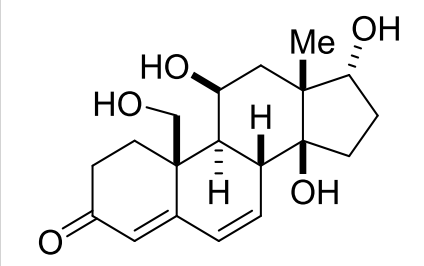

Compound 4

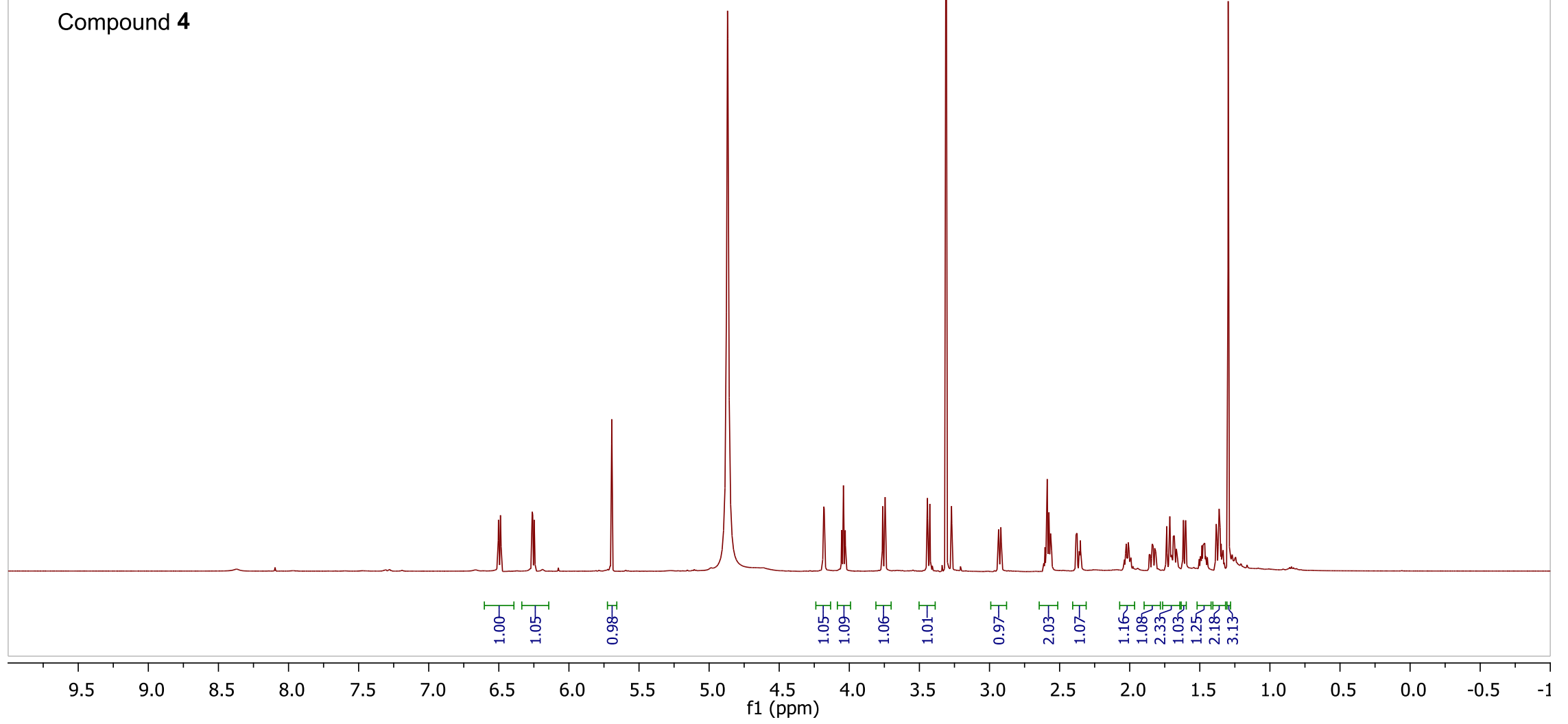




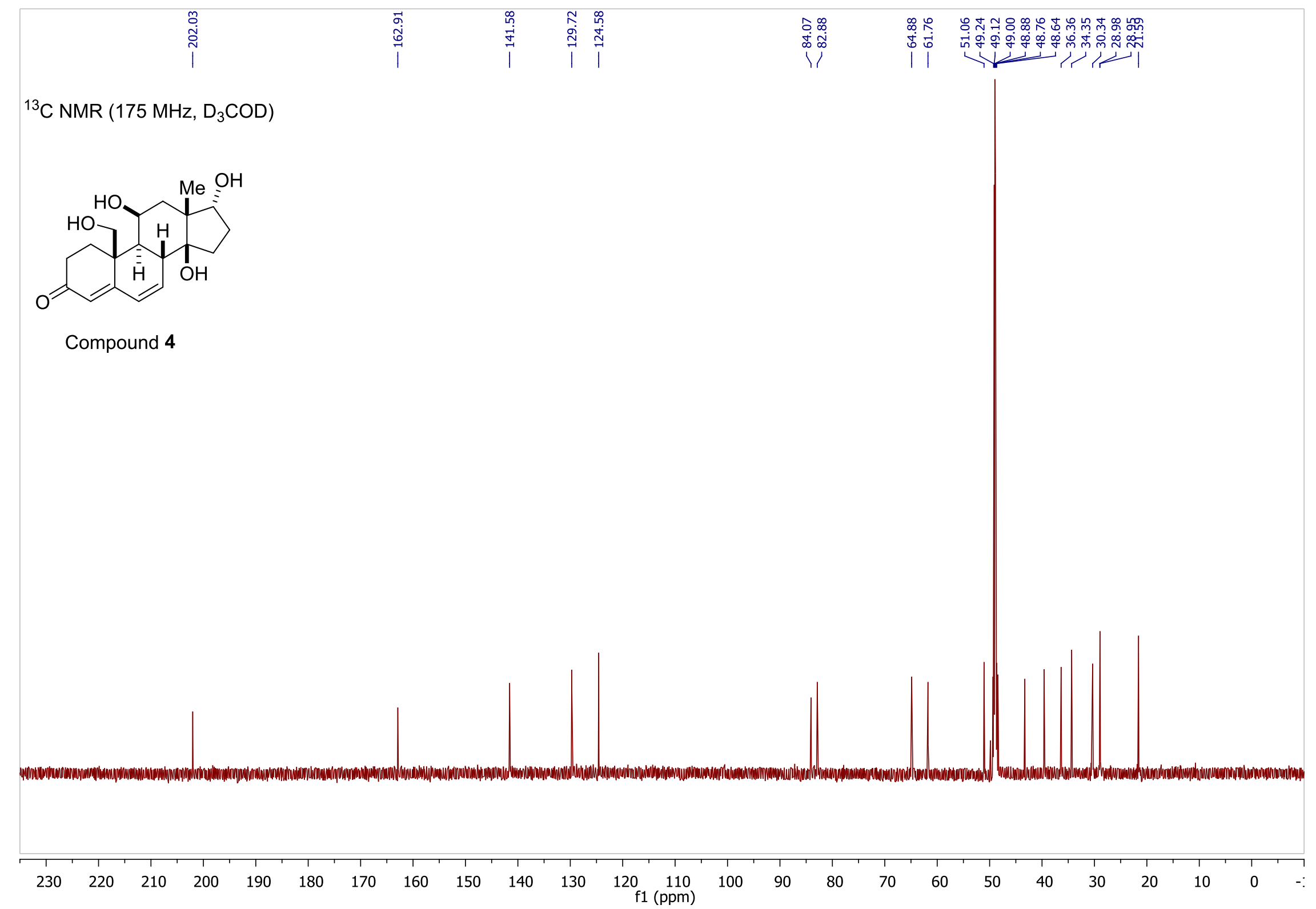




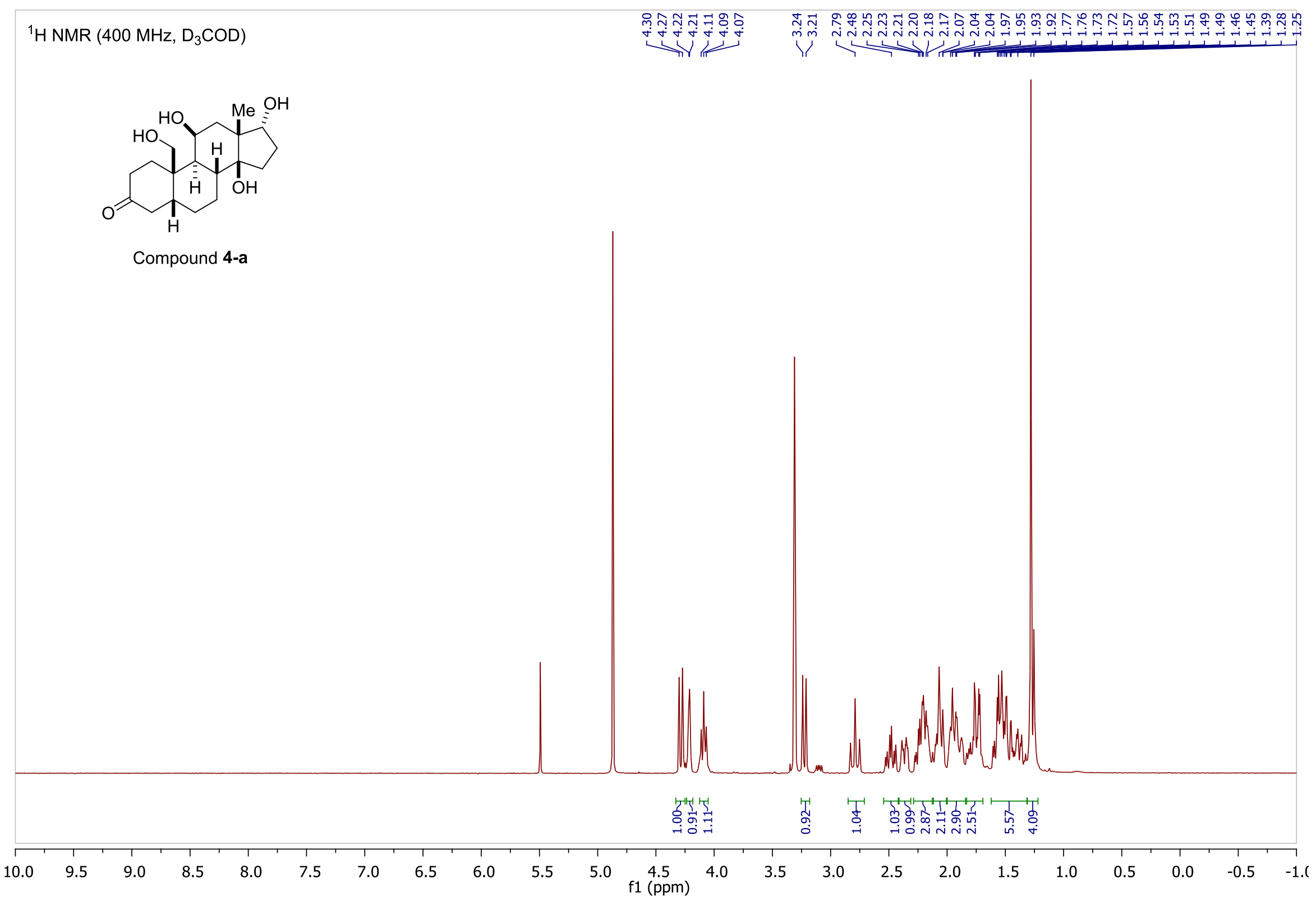




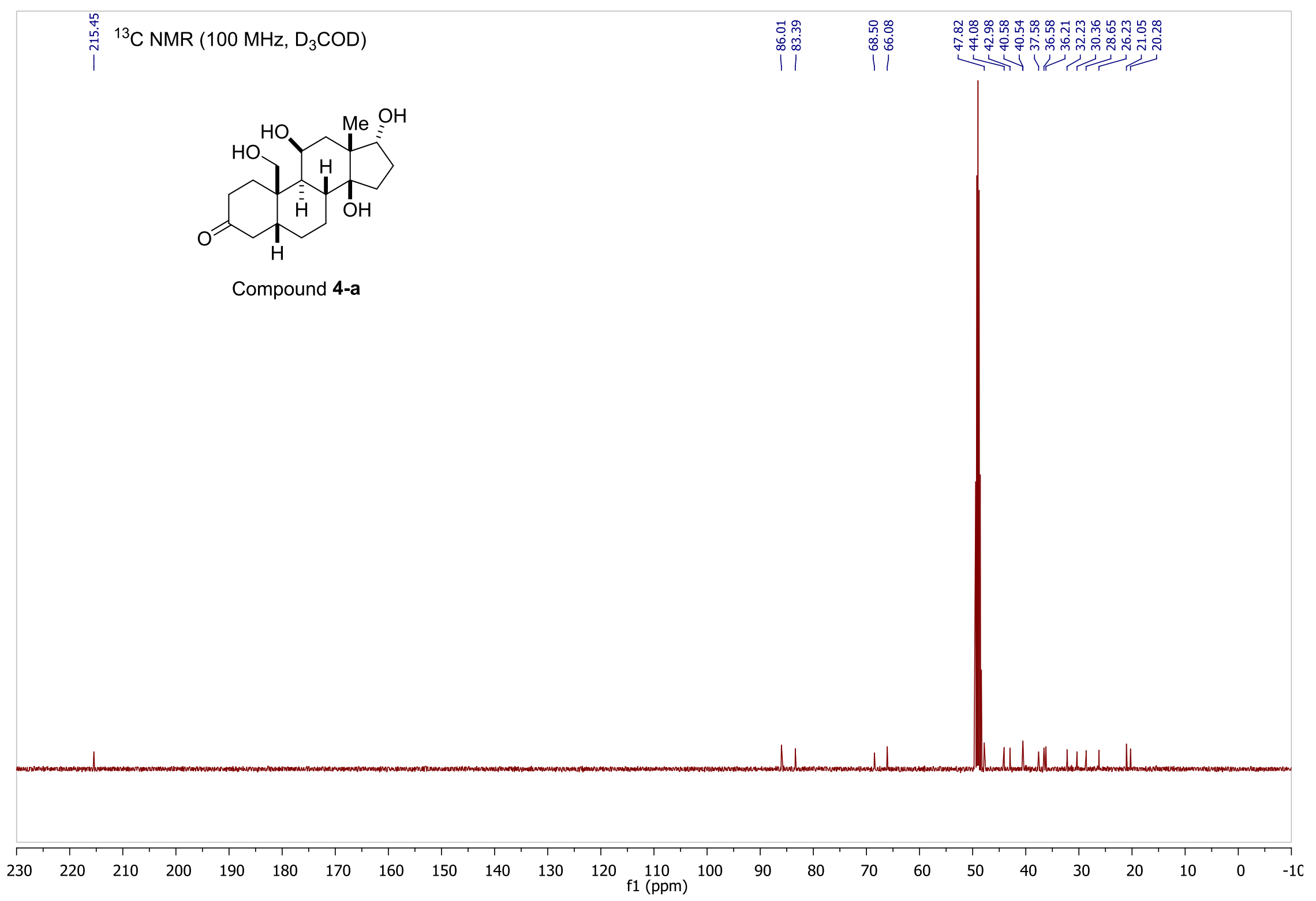




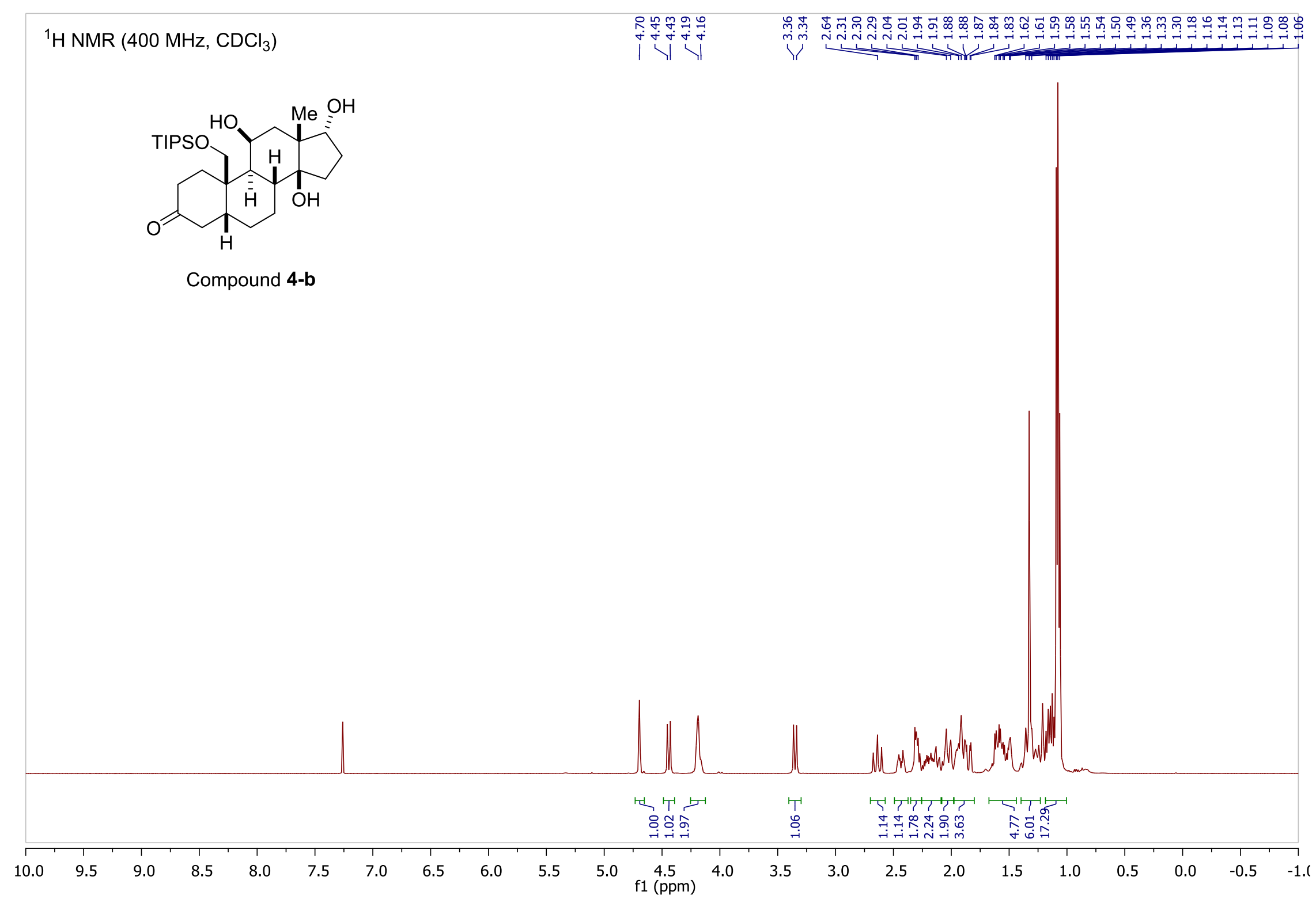




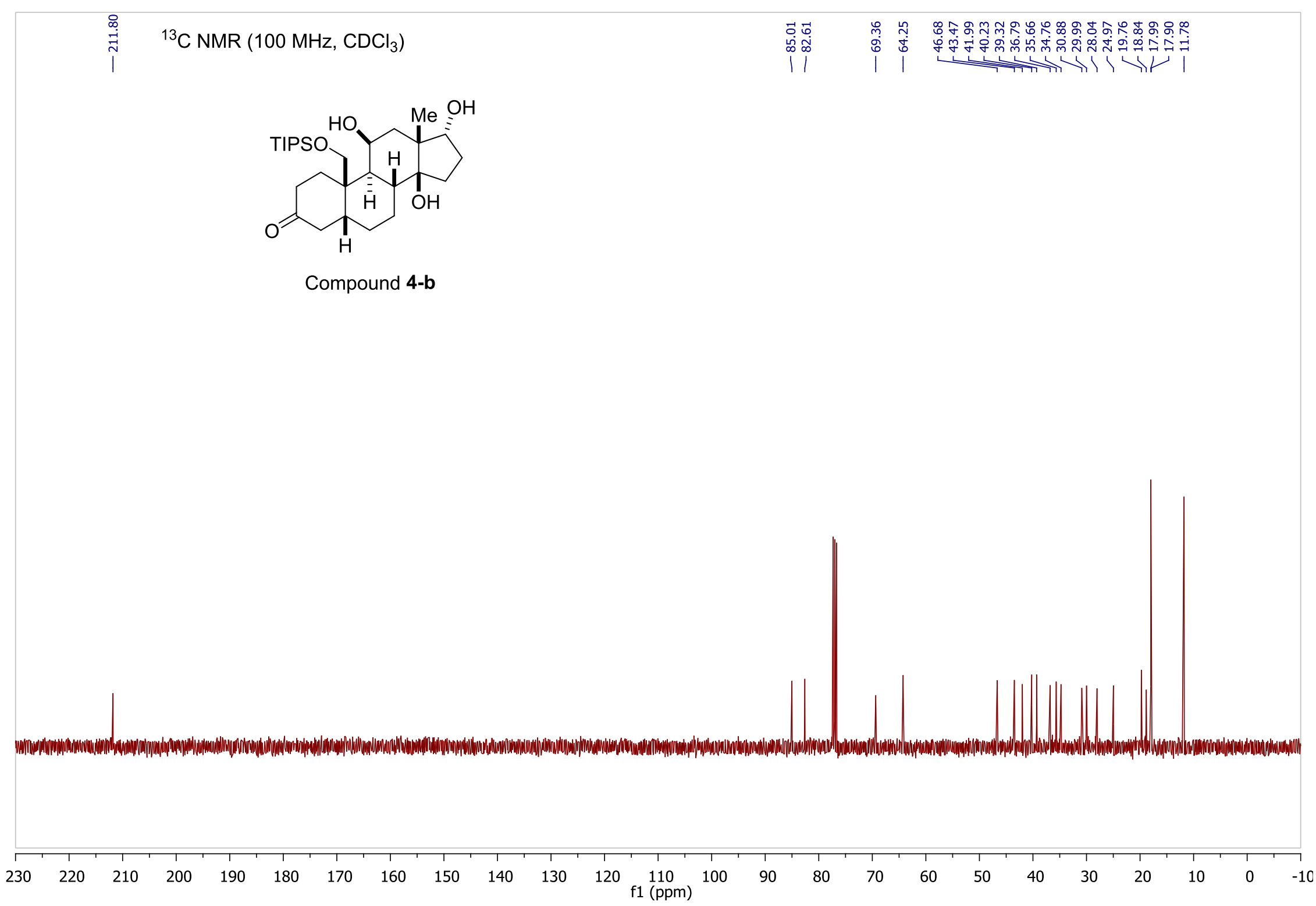




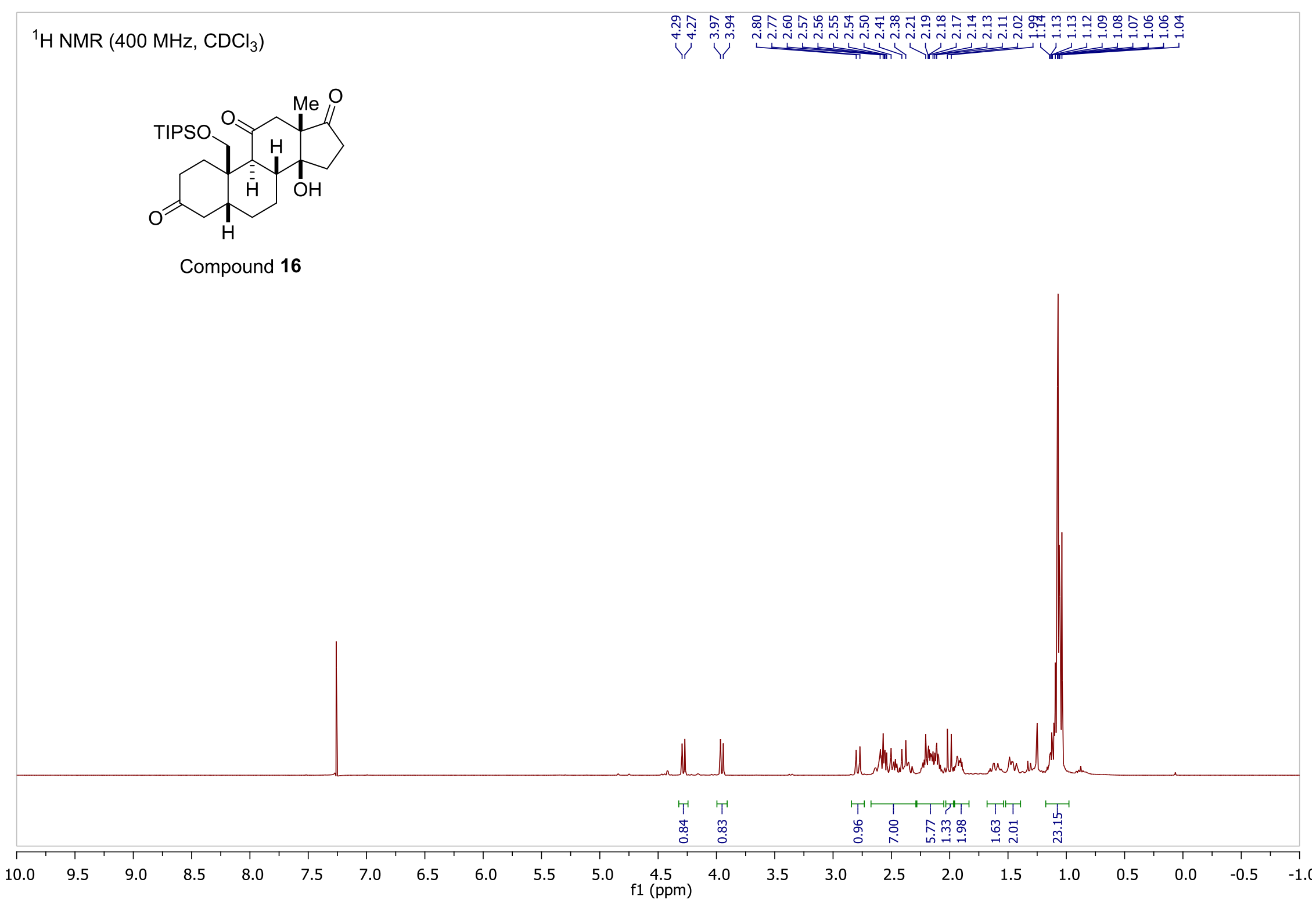




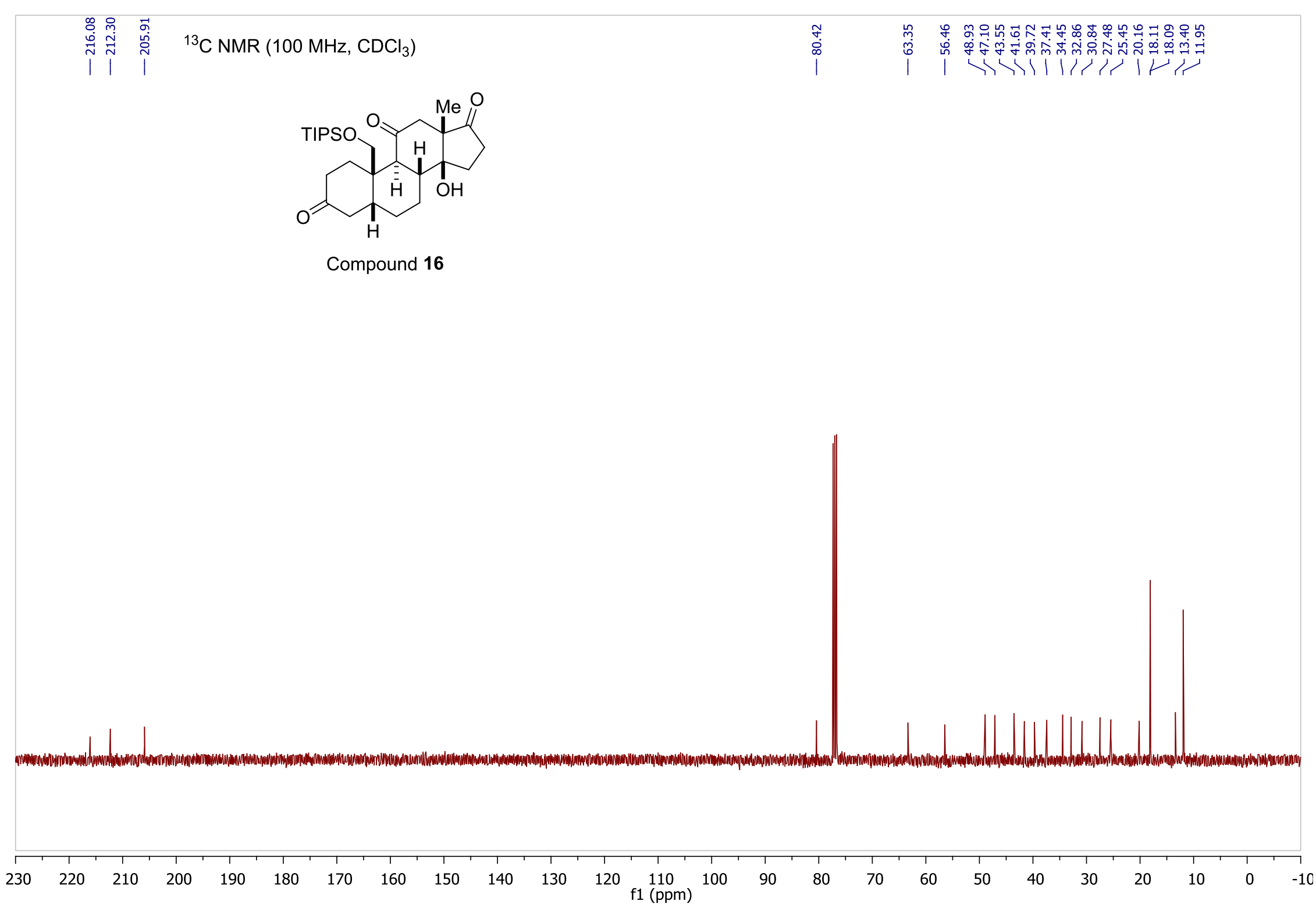




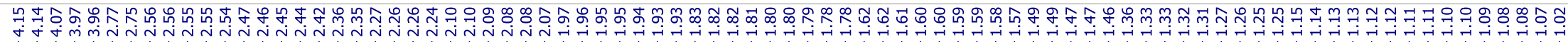

${ }^{1} \mathrm{H} \mathrm{NMR}\left(700 \mathrm{MHz}, \mathrm{CDCl}_{3}\right)$

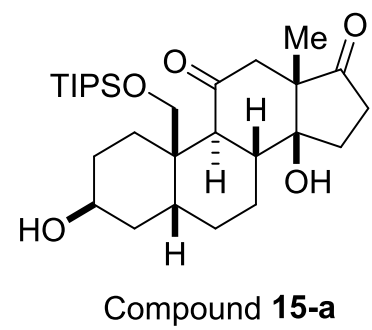

Compound 15-a

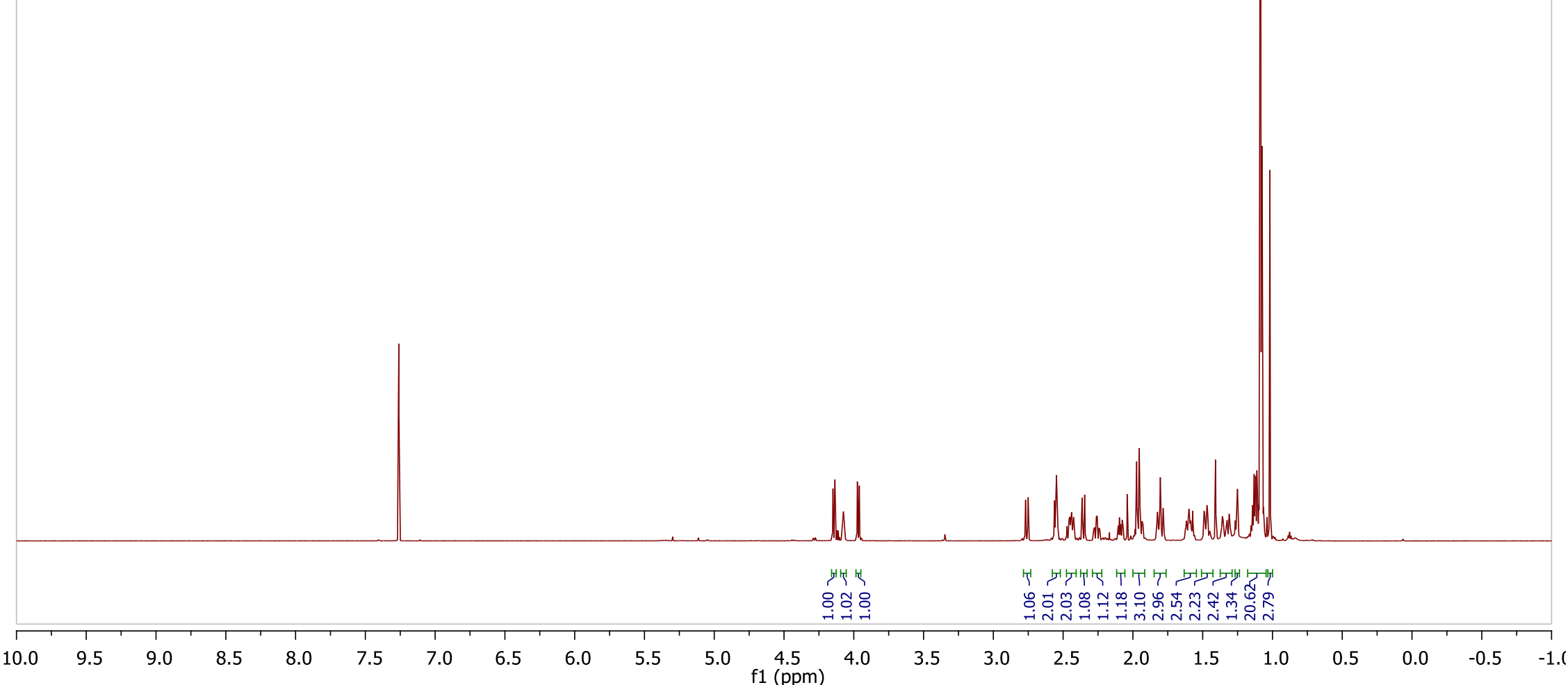




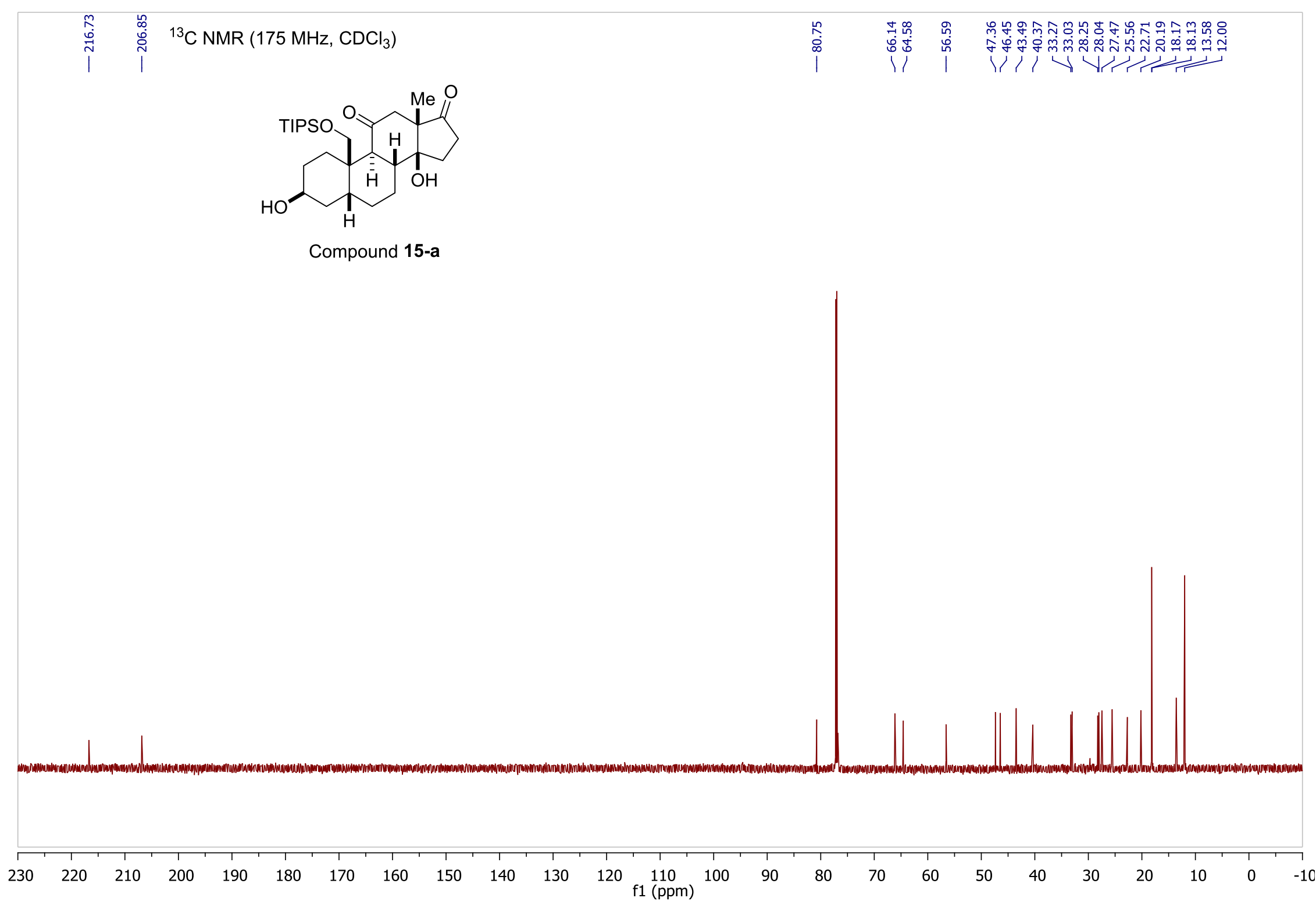




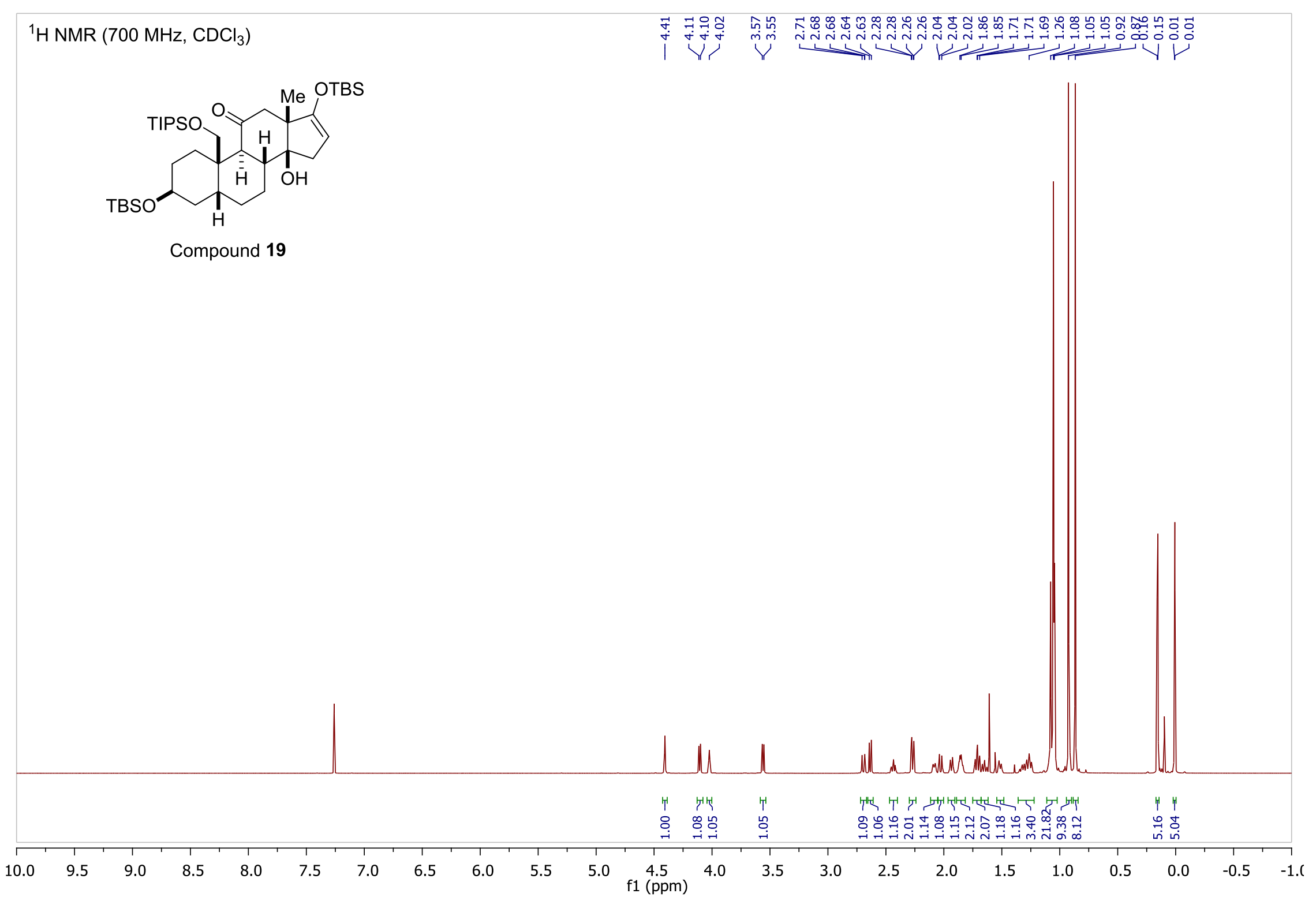




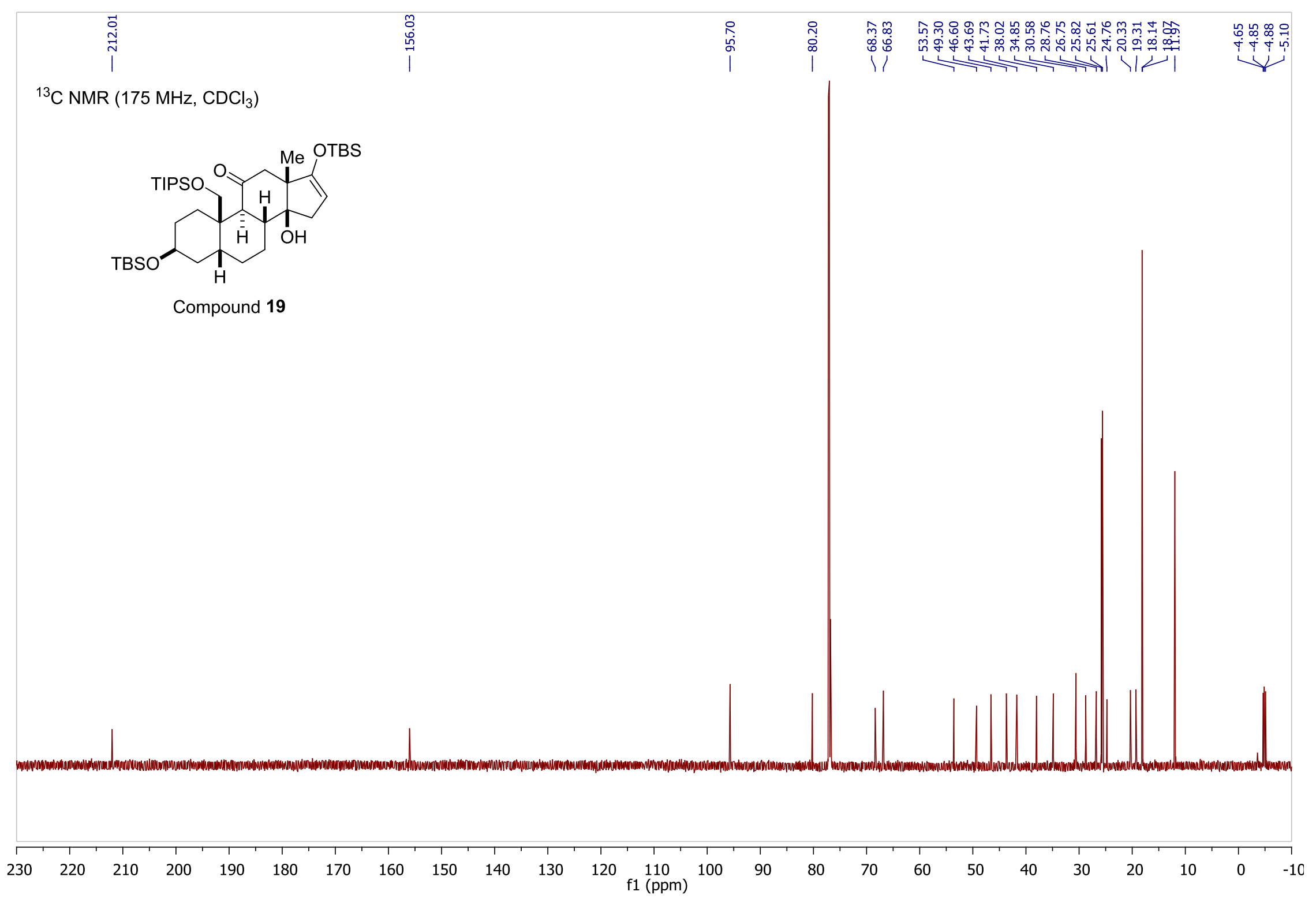




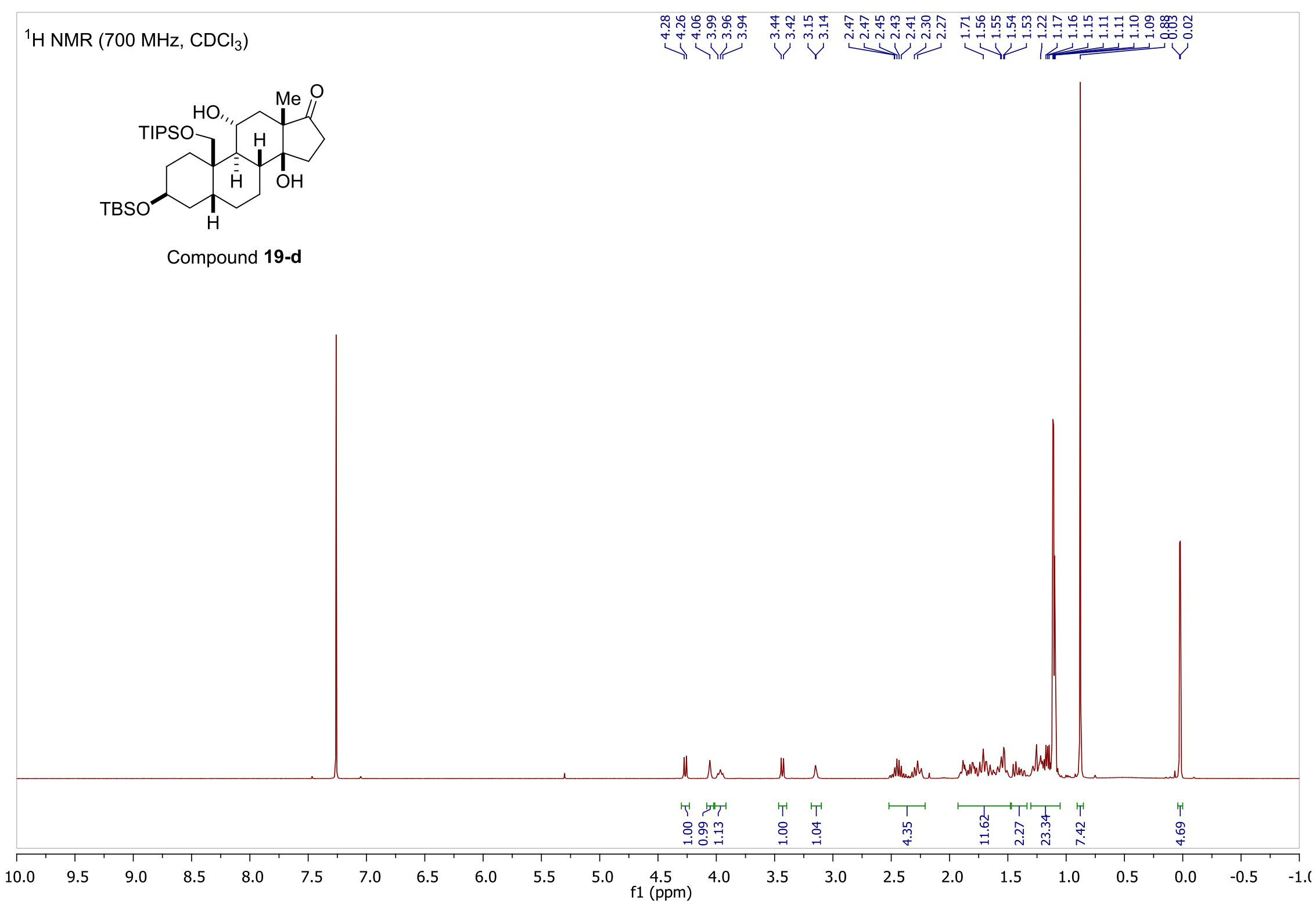




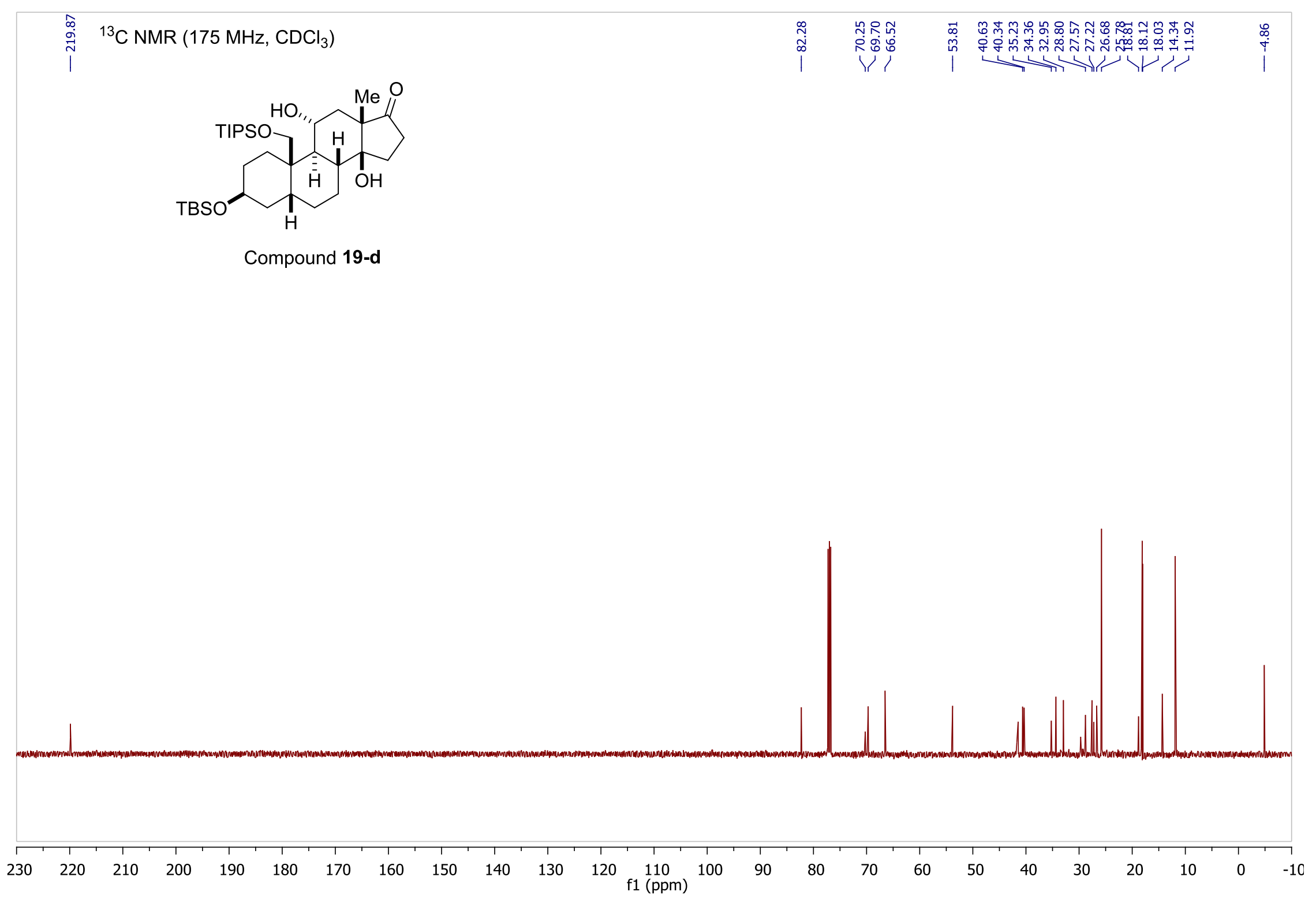




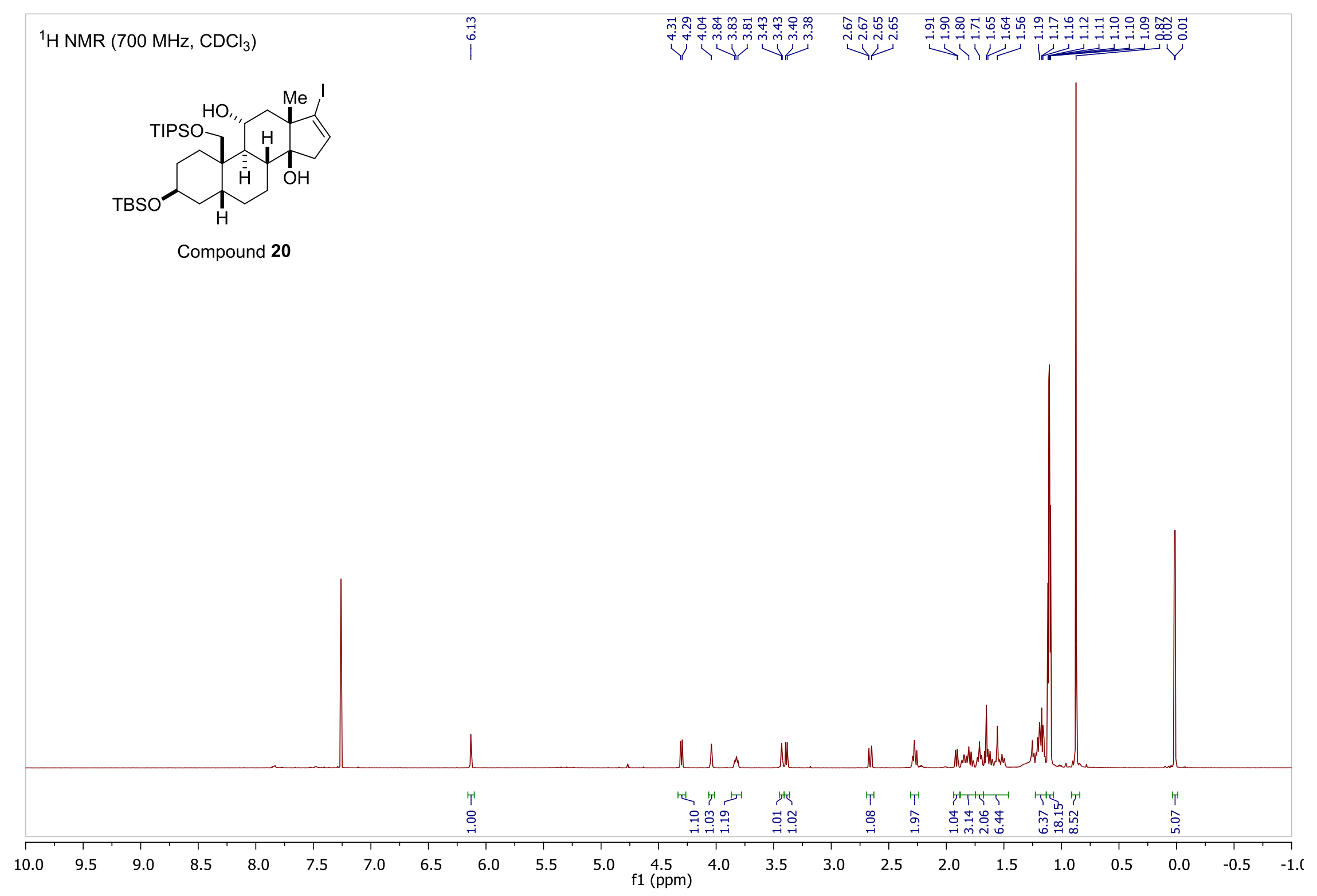




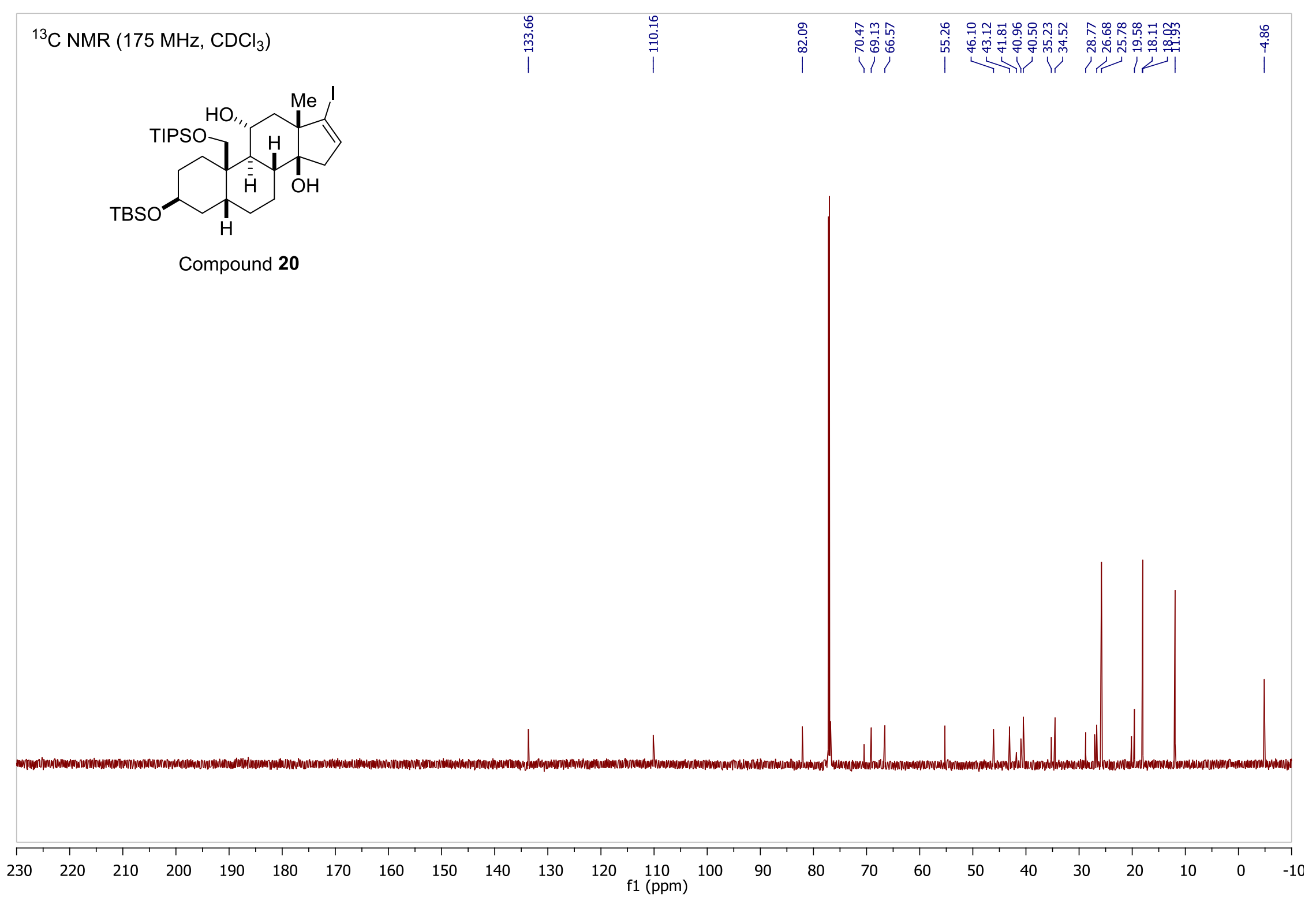




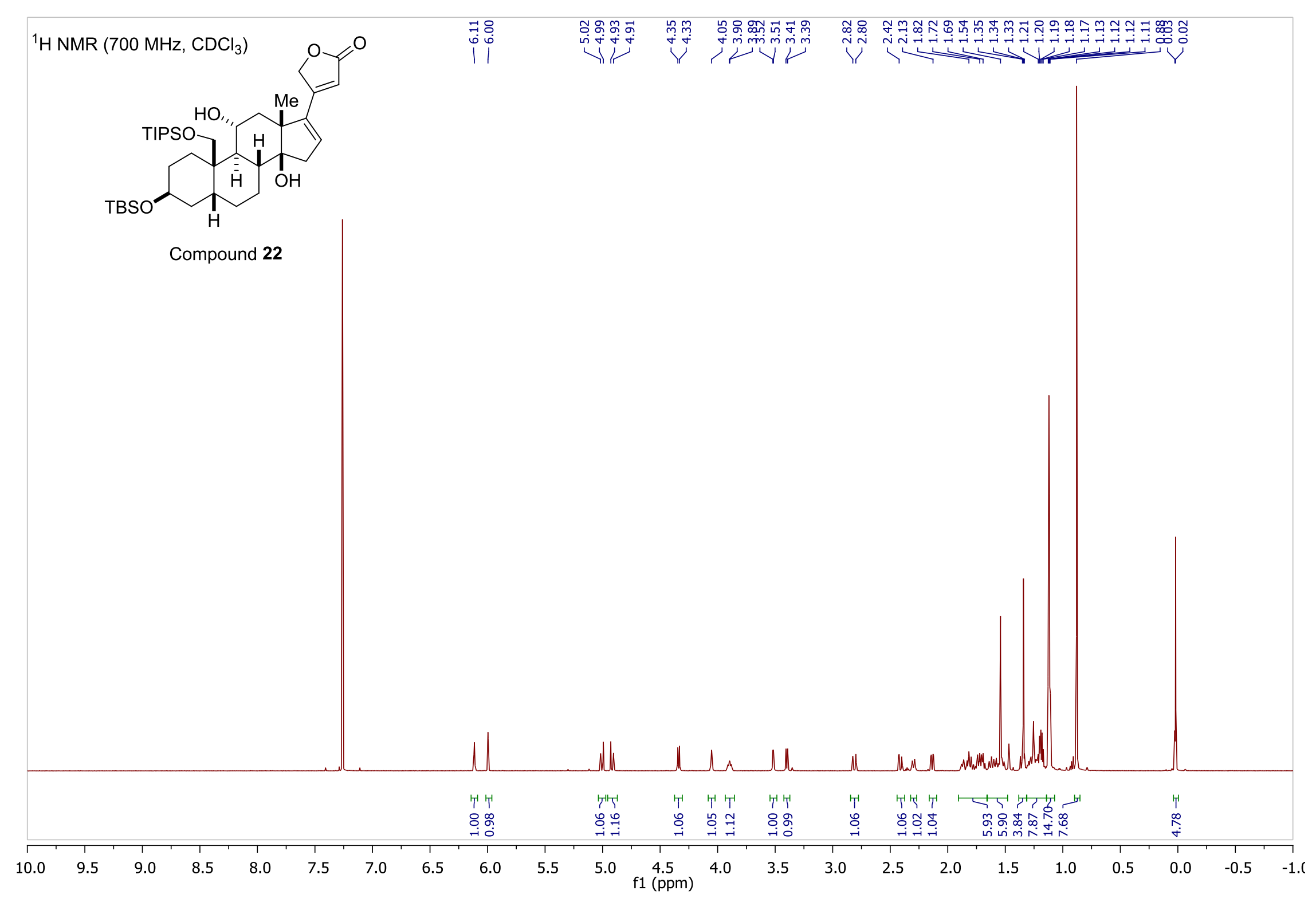




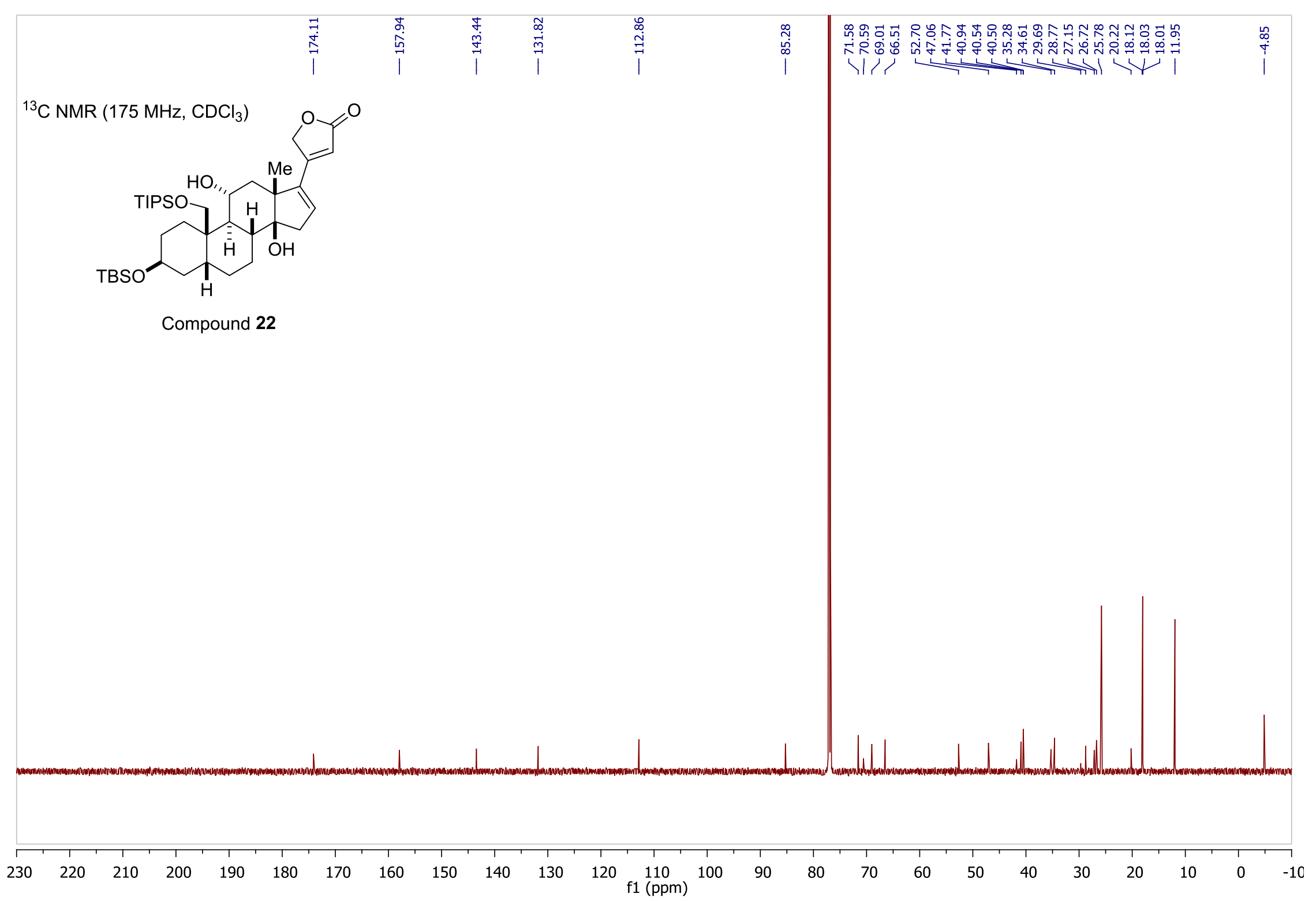




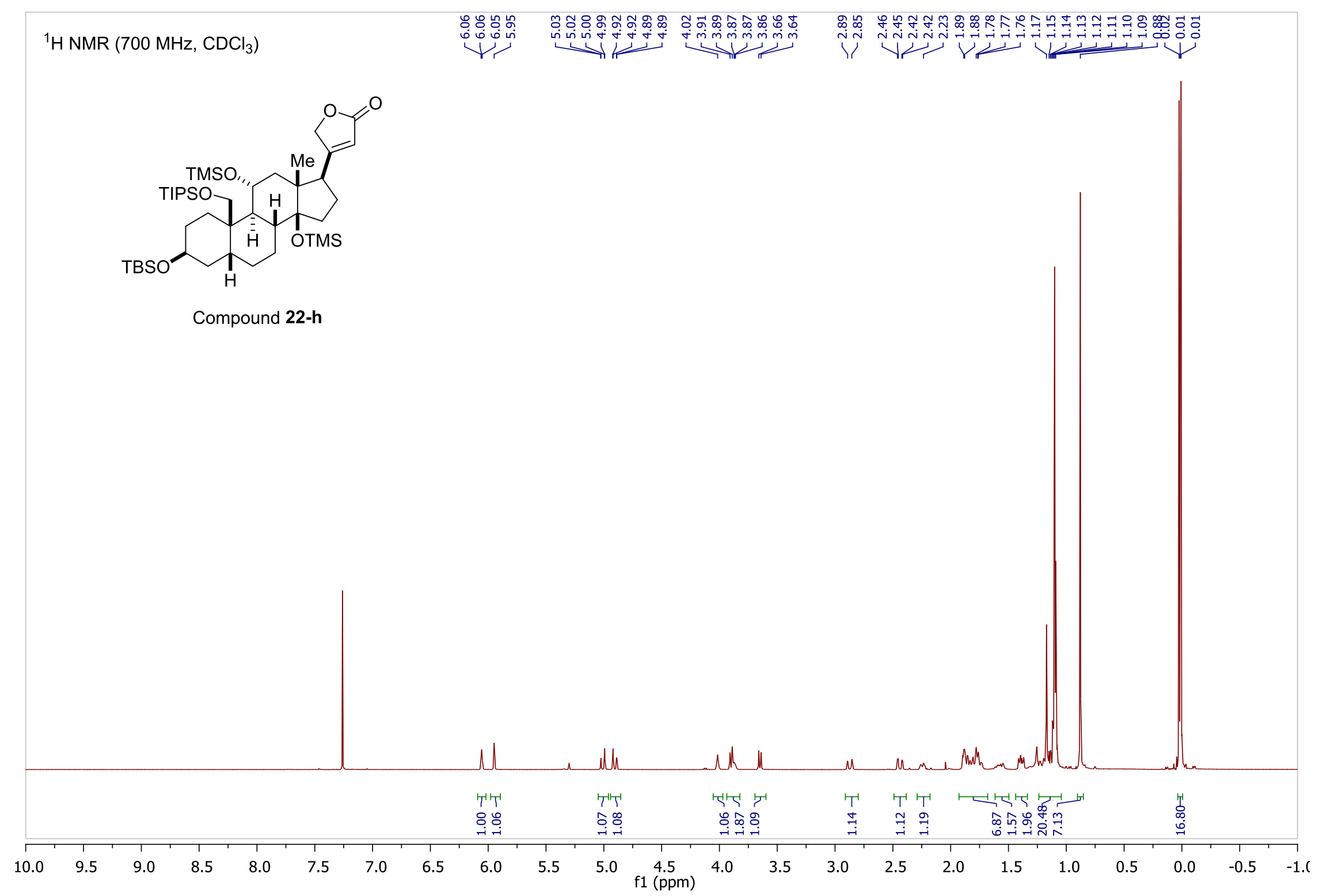




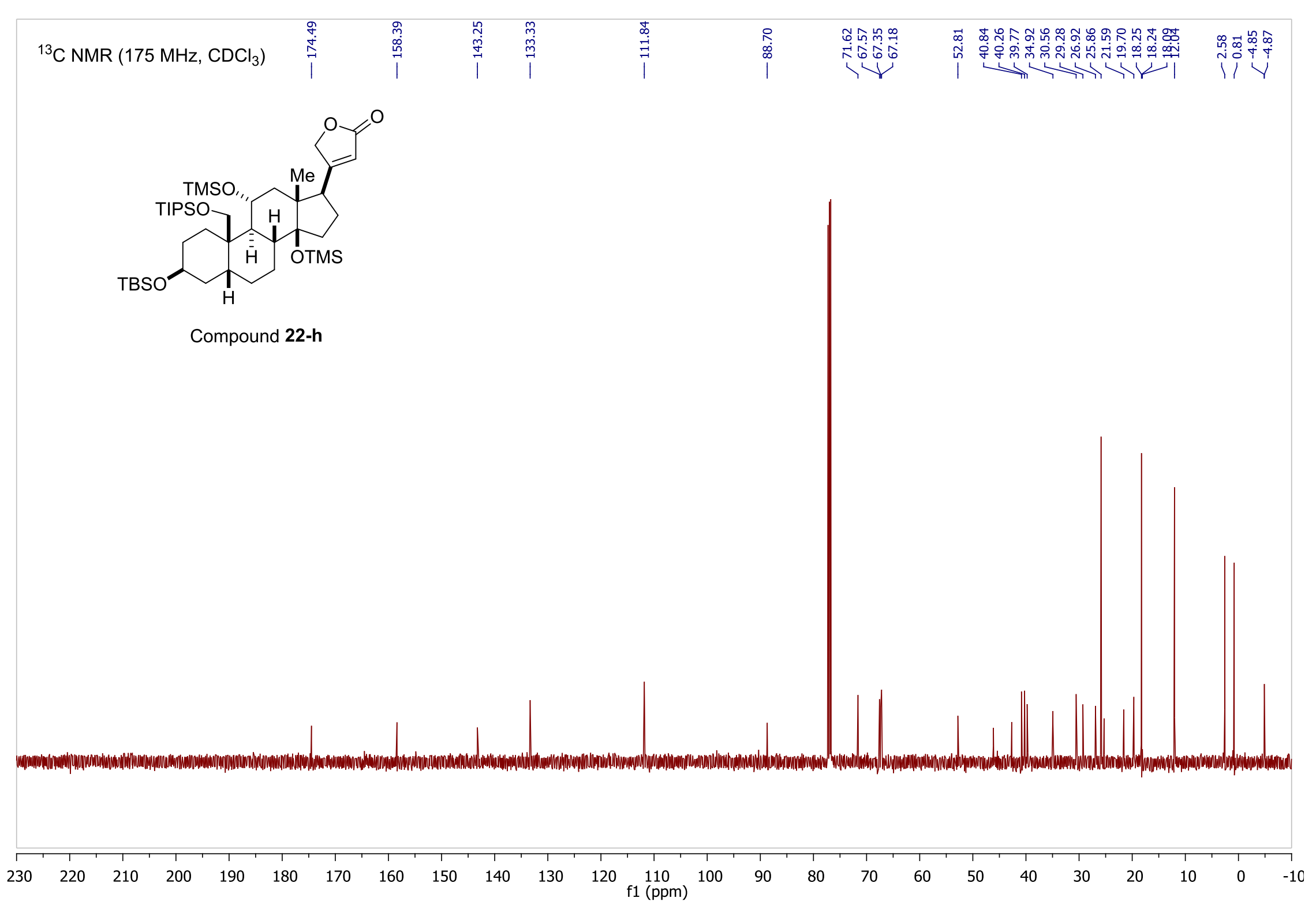




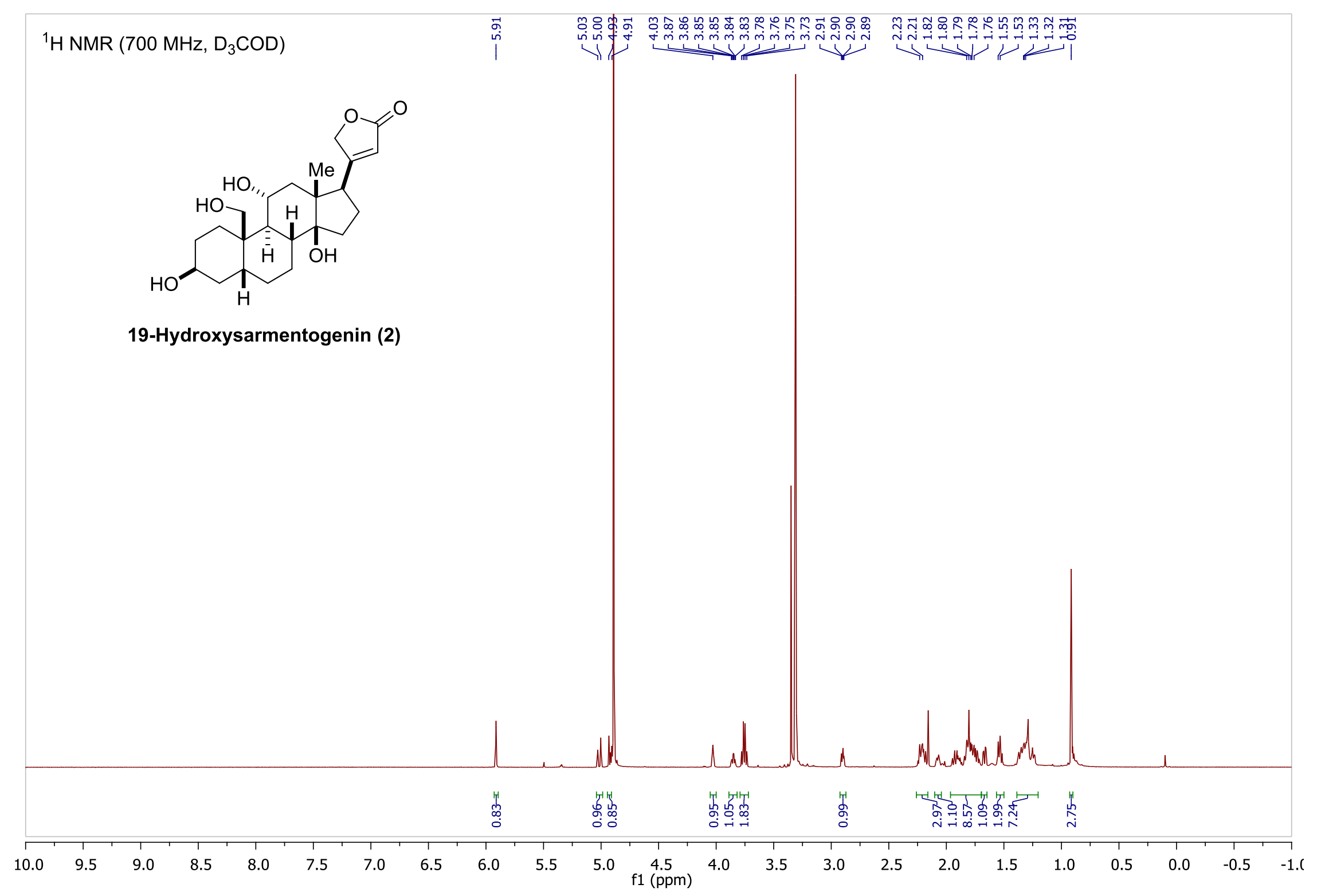




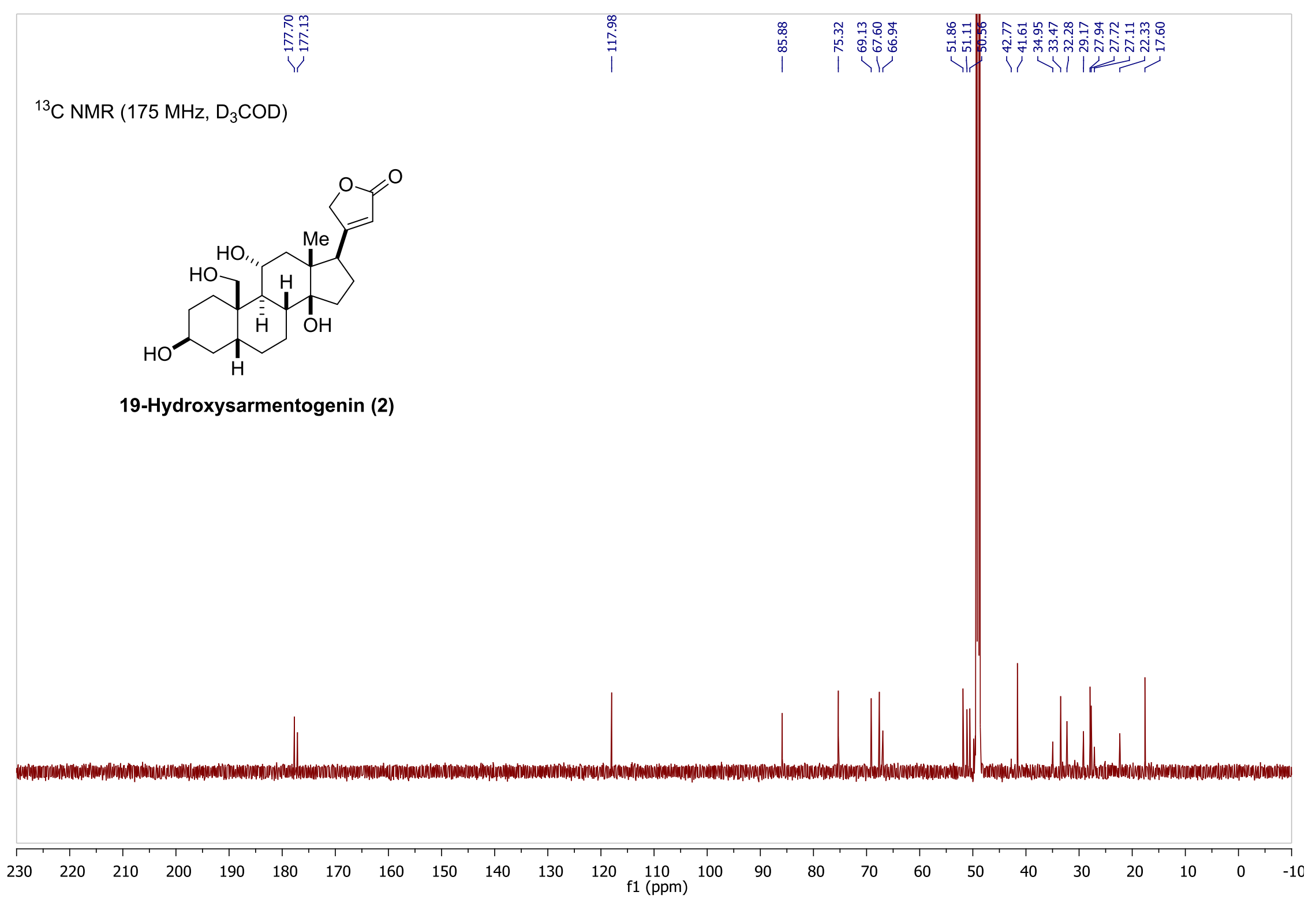




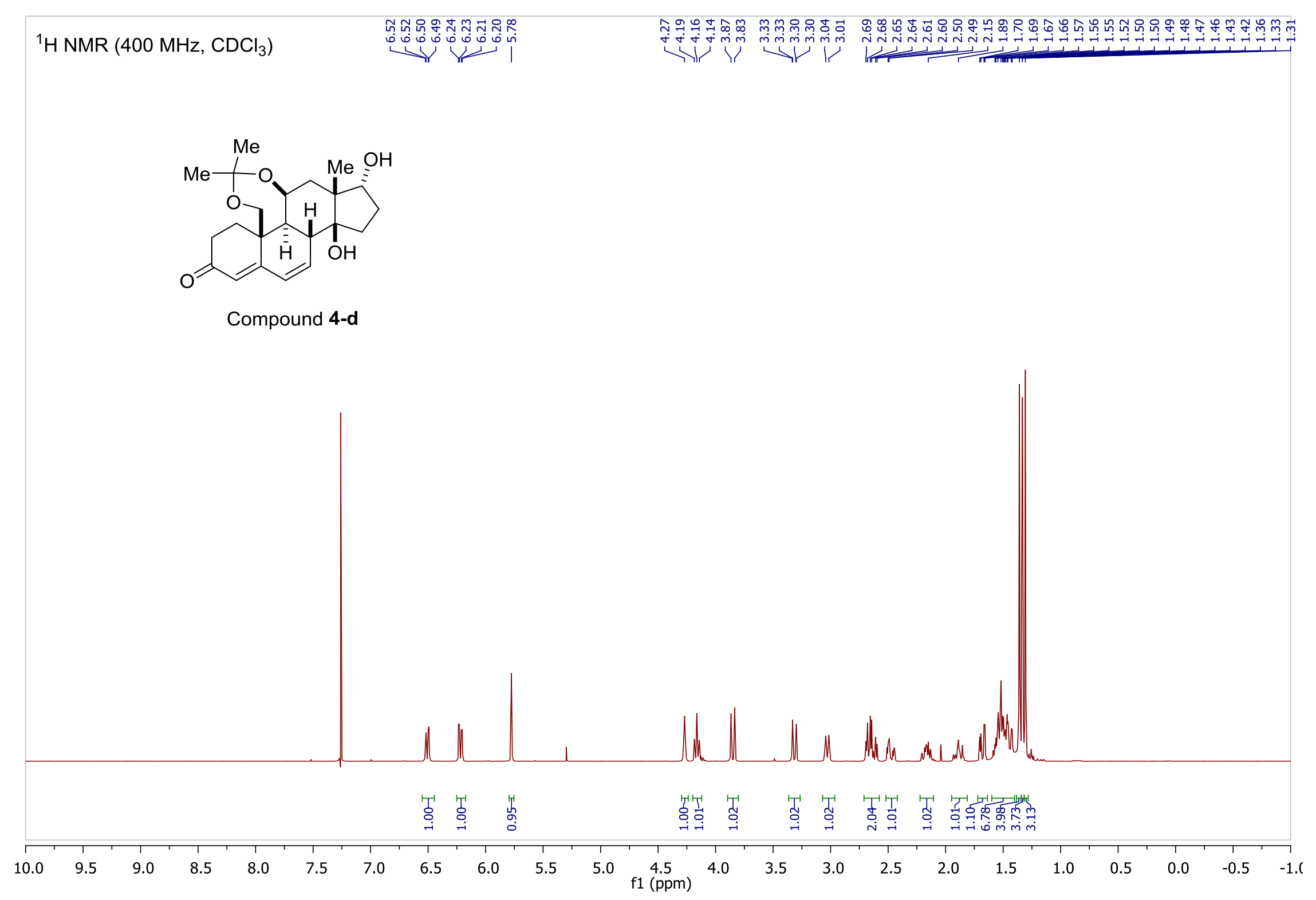




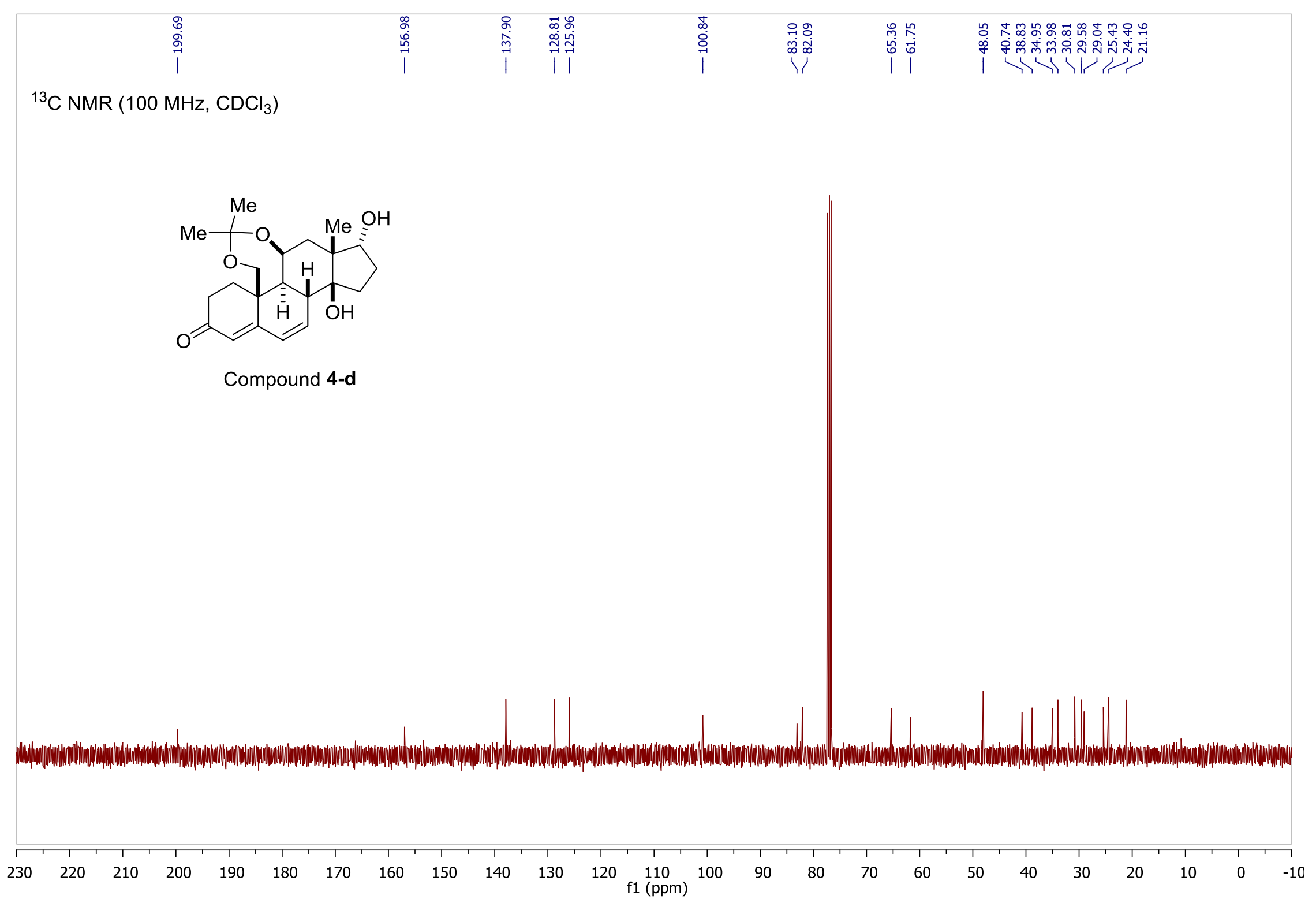


HSQC
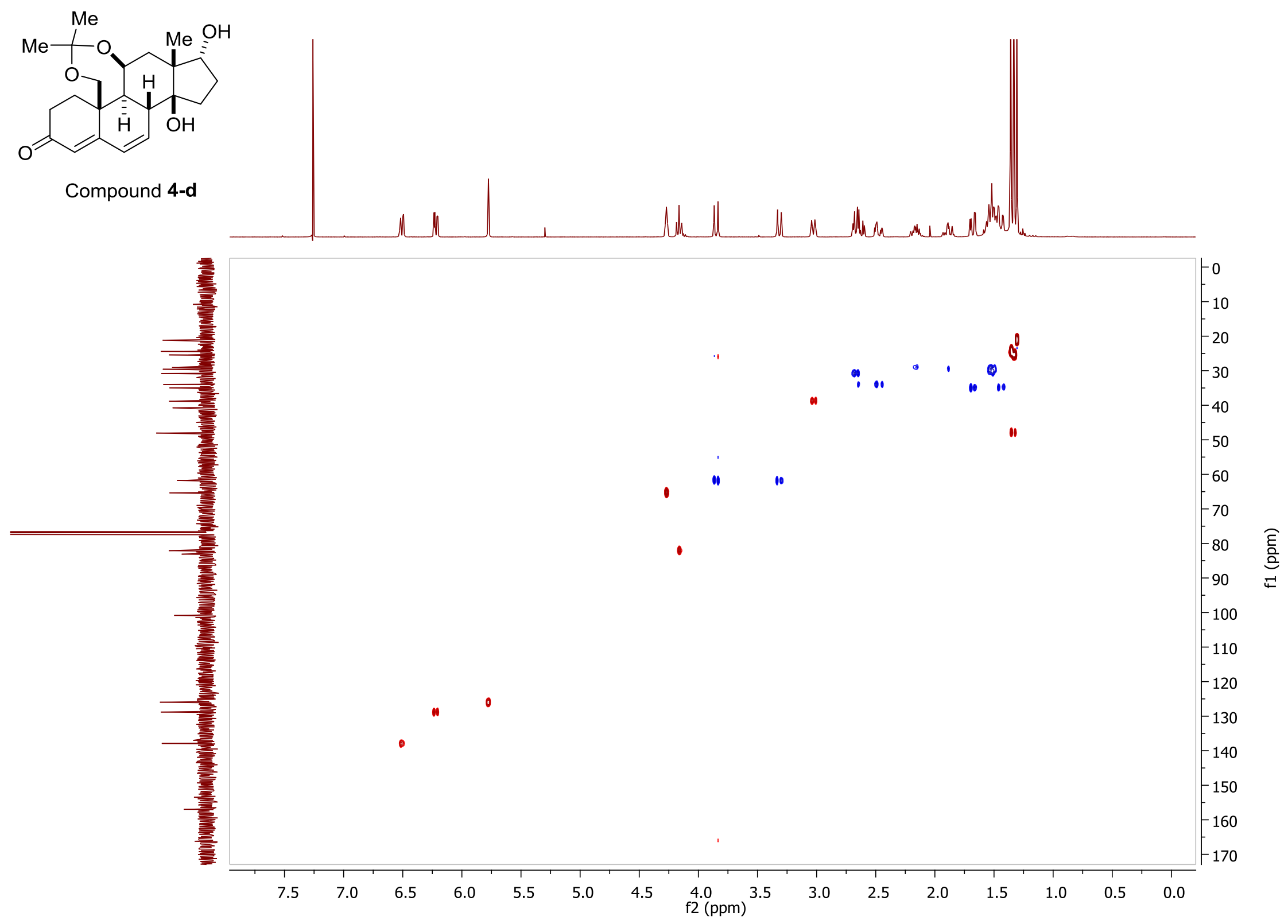


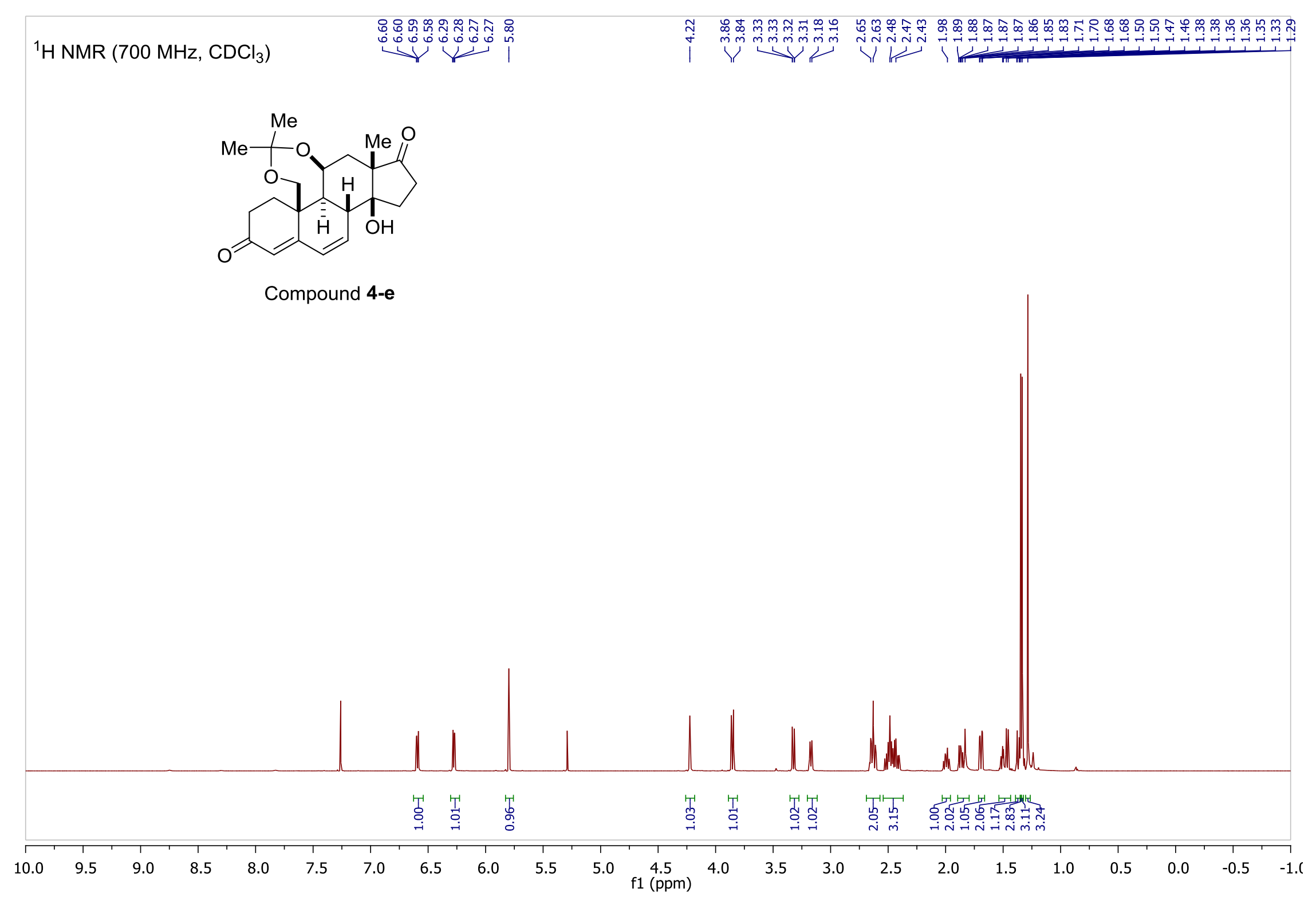




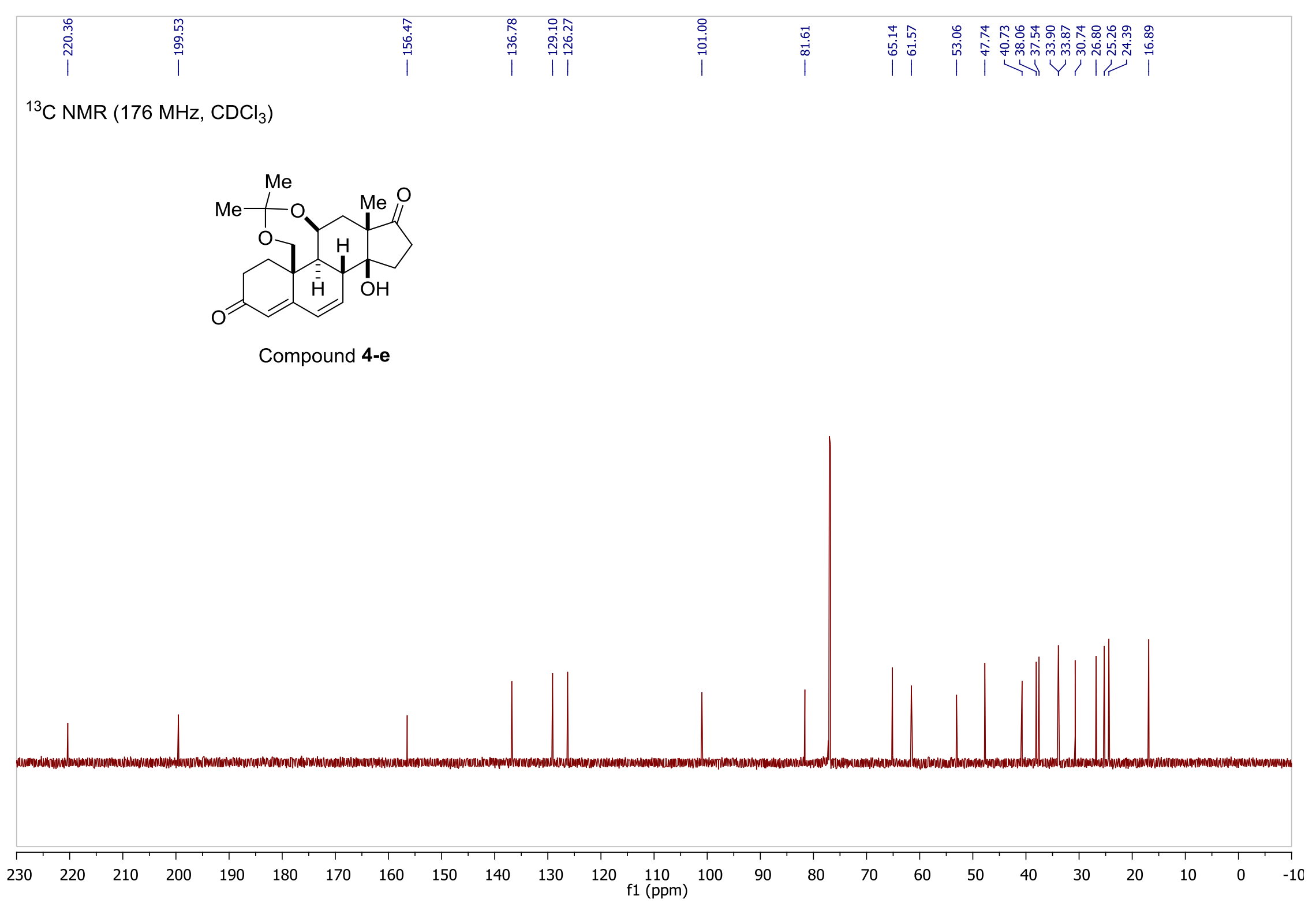


HSQC

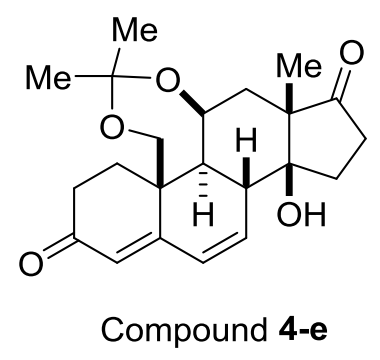

Compound 4-e

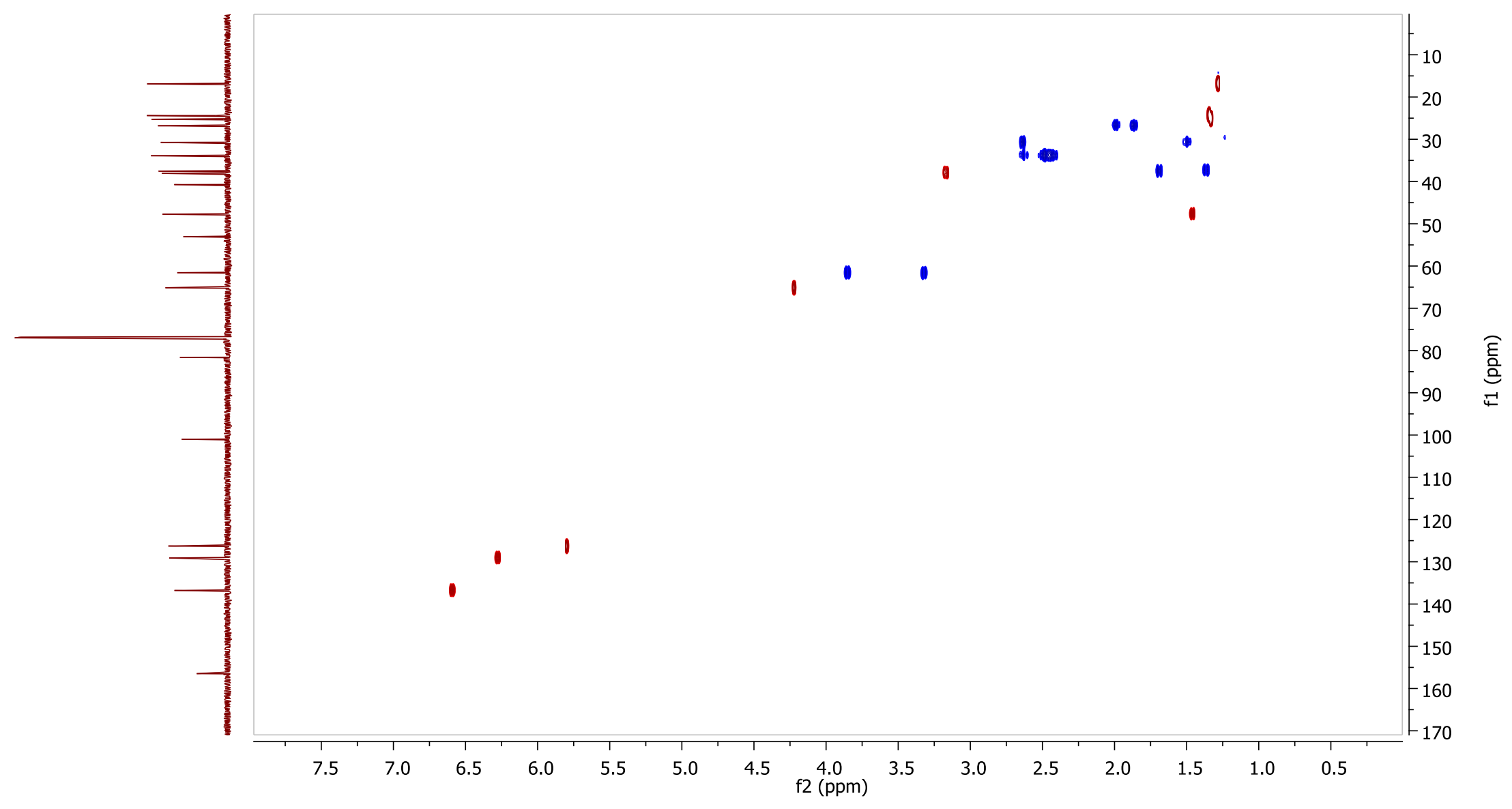




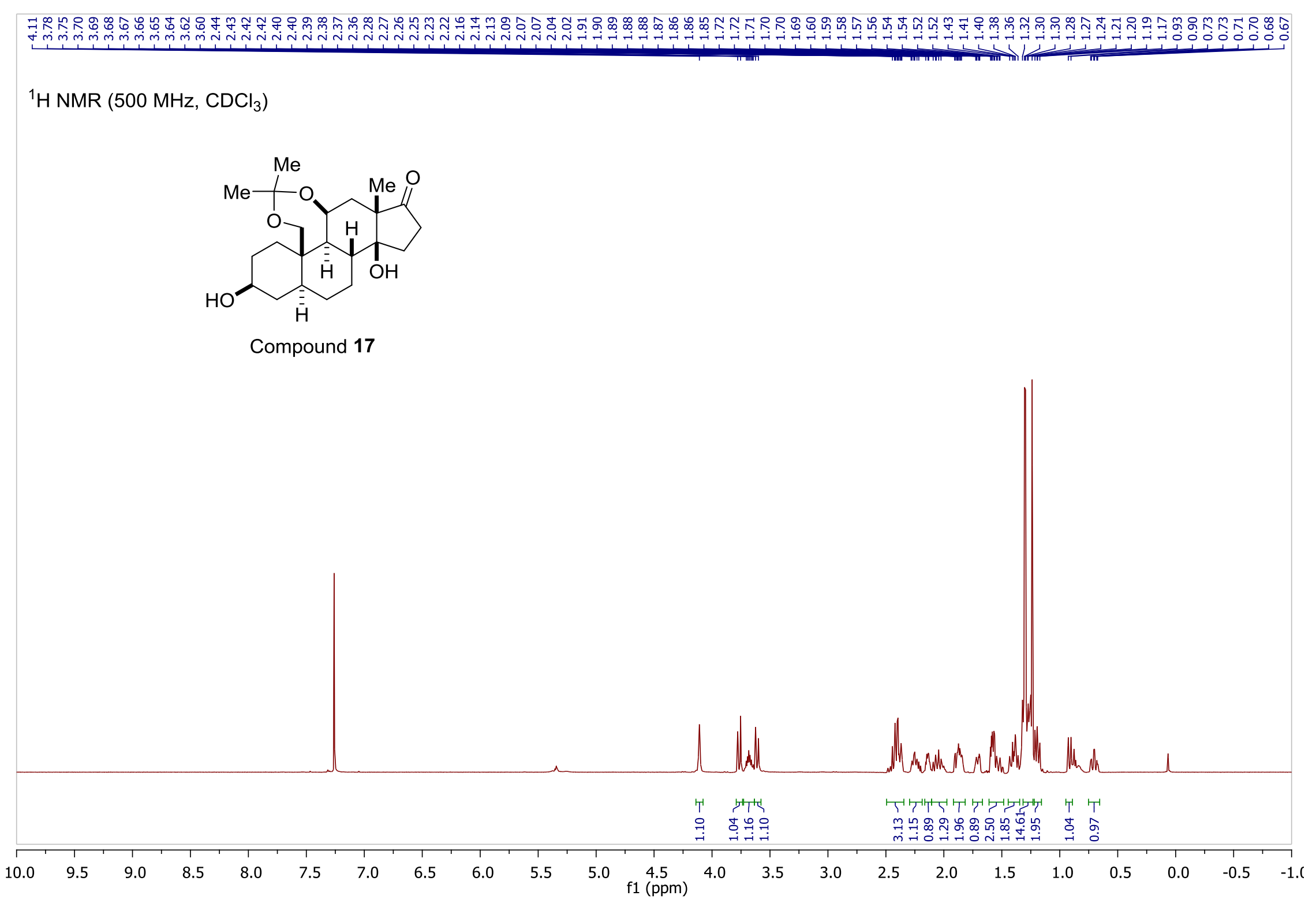




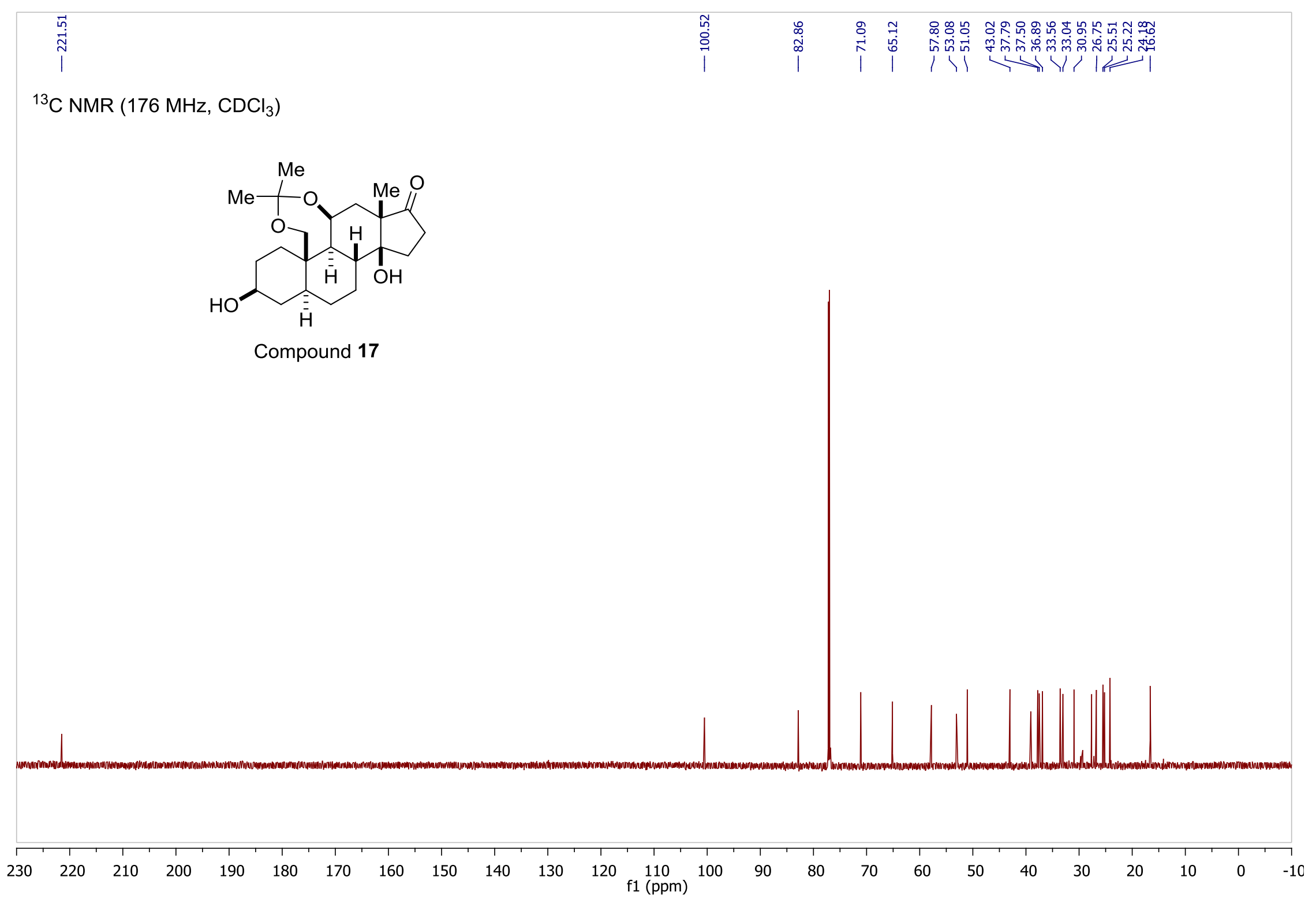




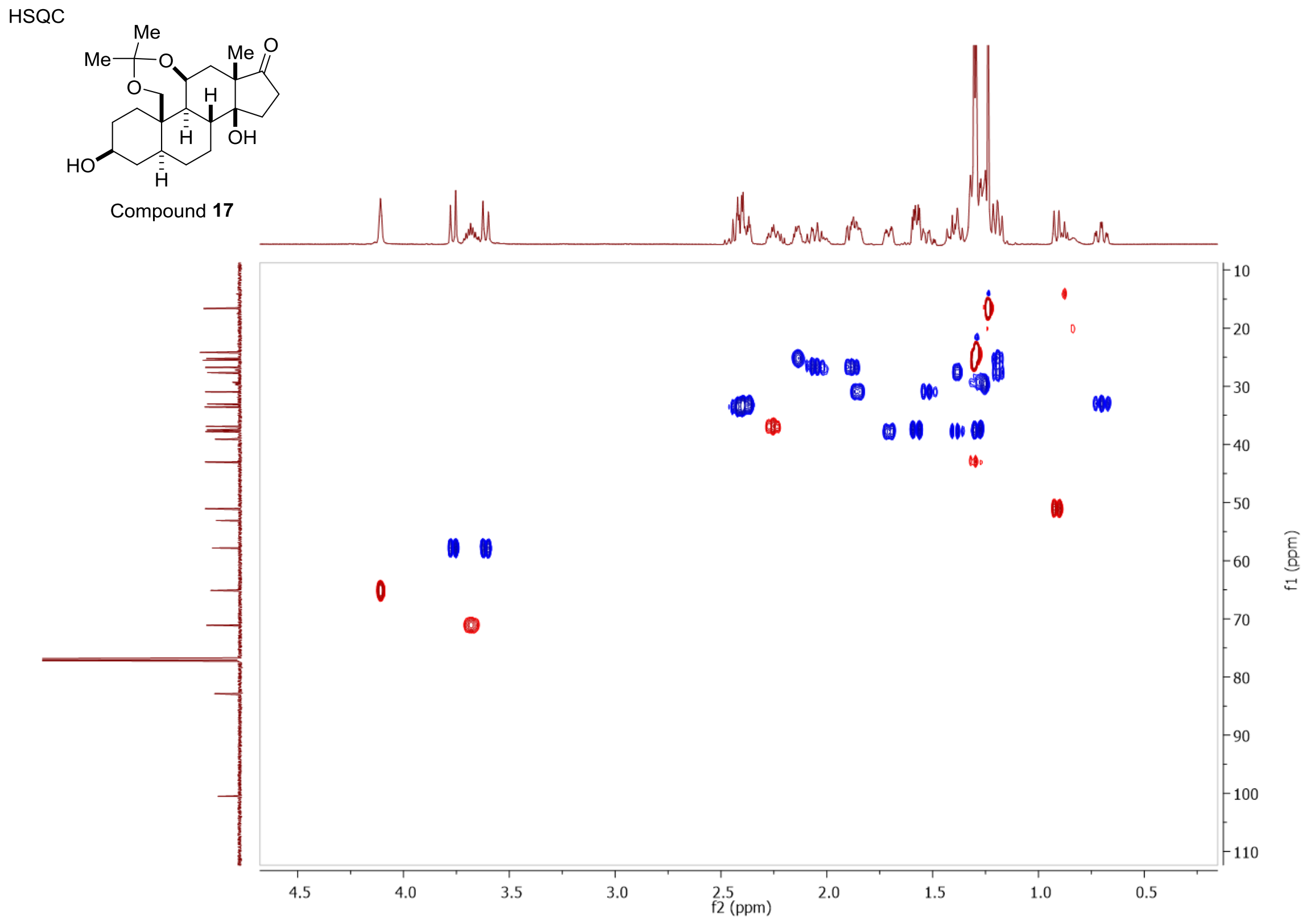




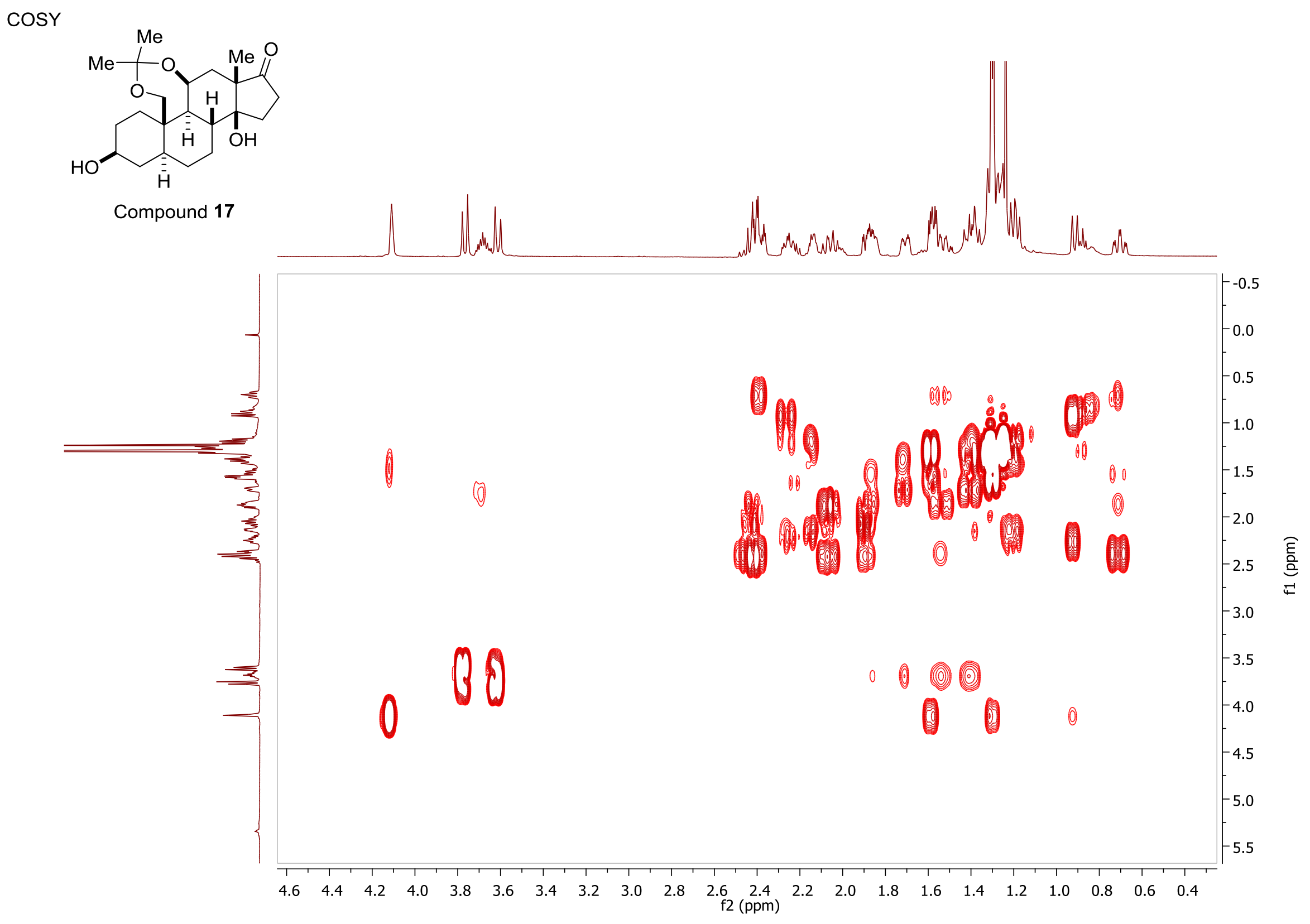




$$
\text { Compound } 17
$$

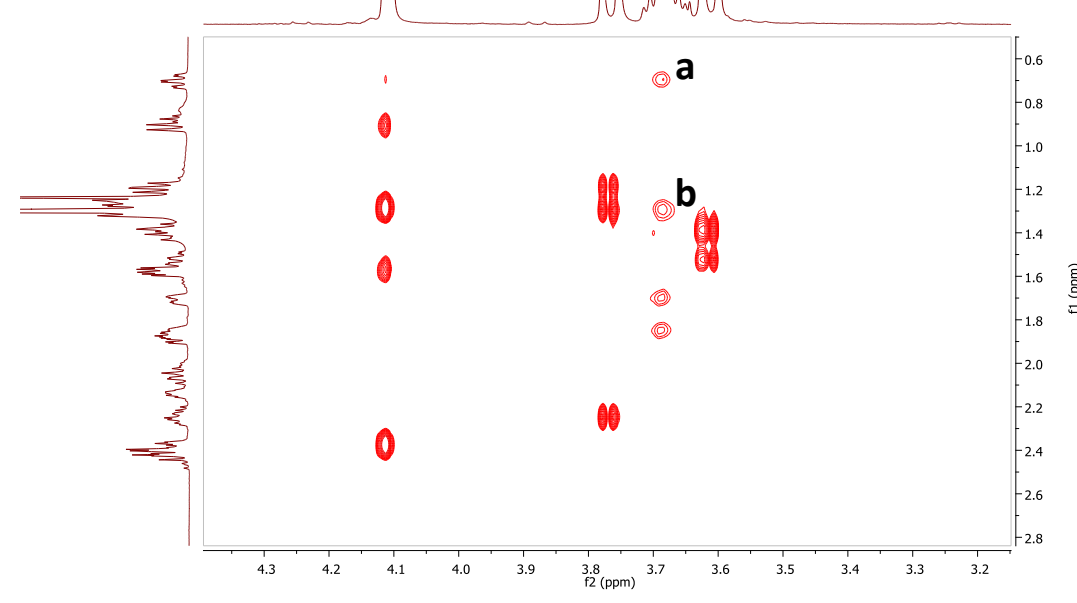

H1- $\alpha$

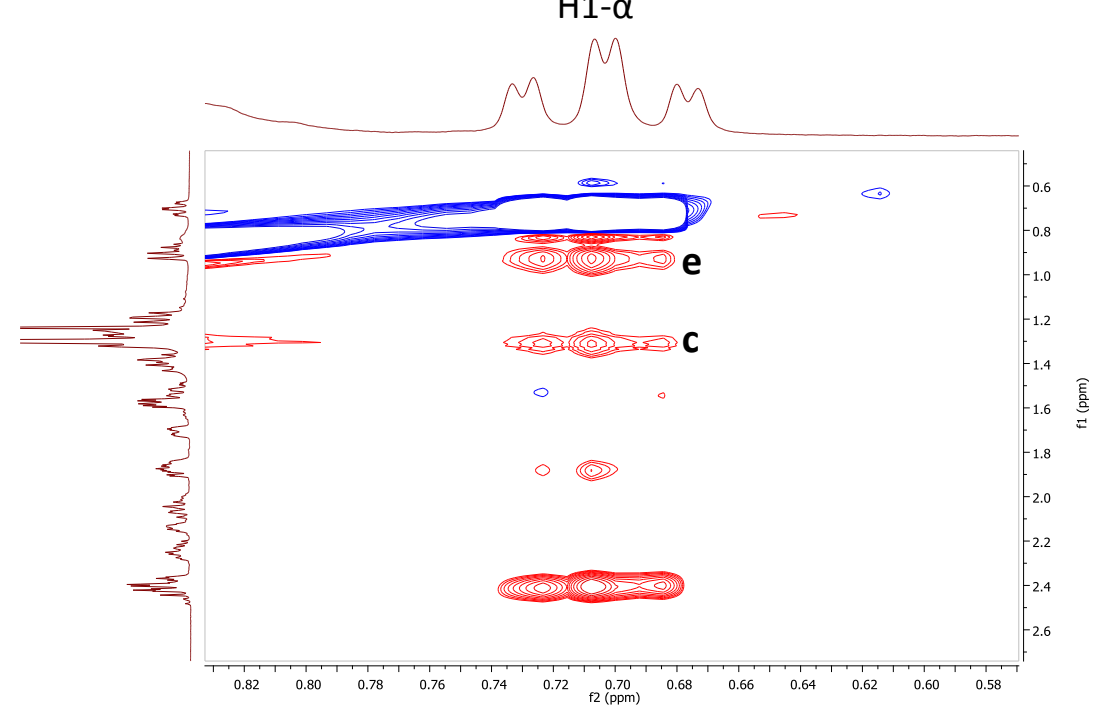

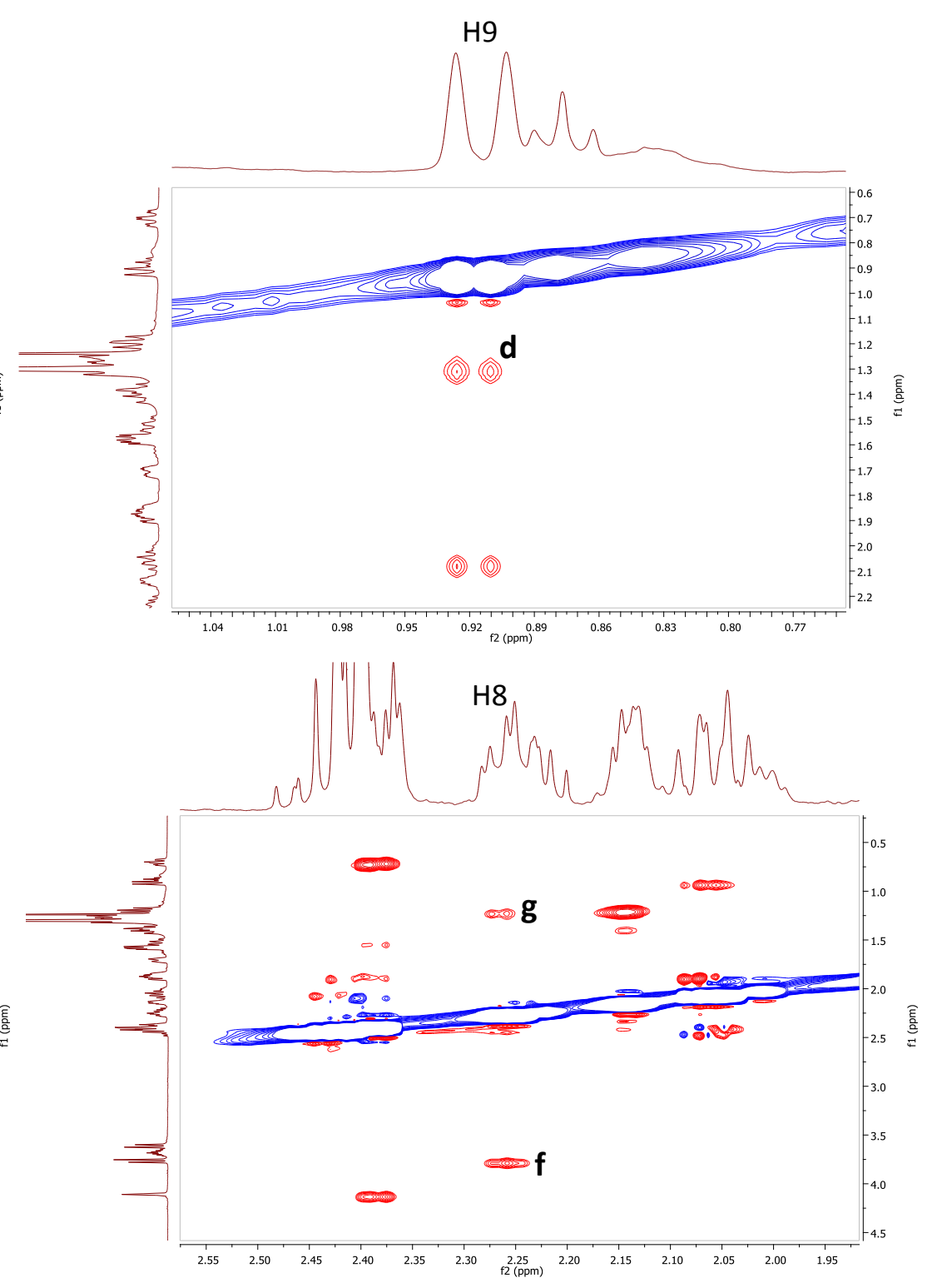


${ }^{1} \mathrm{H}$ NMR $\left(700 \mathrm{MHz}, \mathrm{CDCl}_{3}\right)$

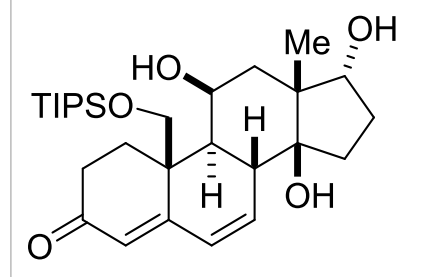

\section{Compound 4-h}

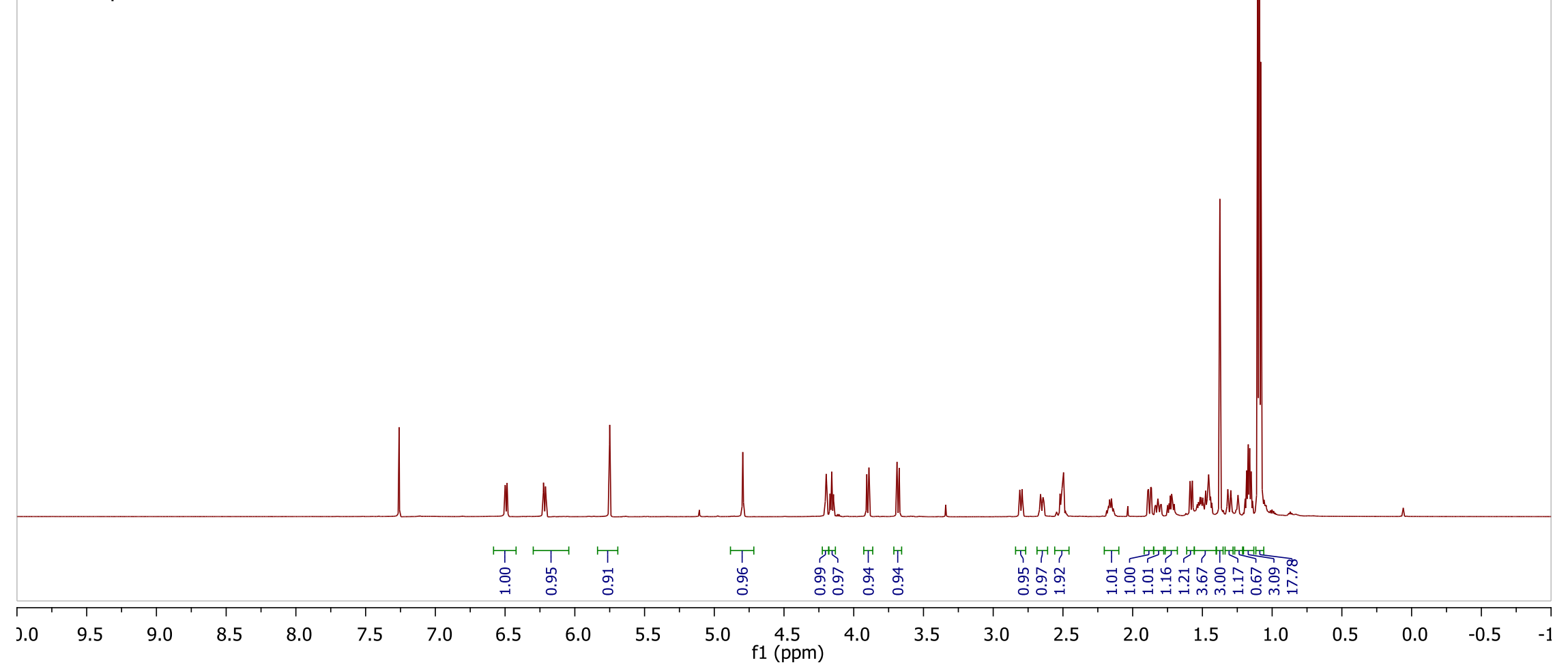




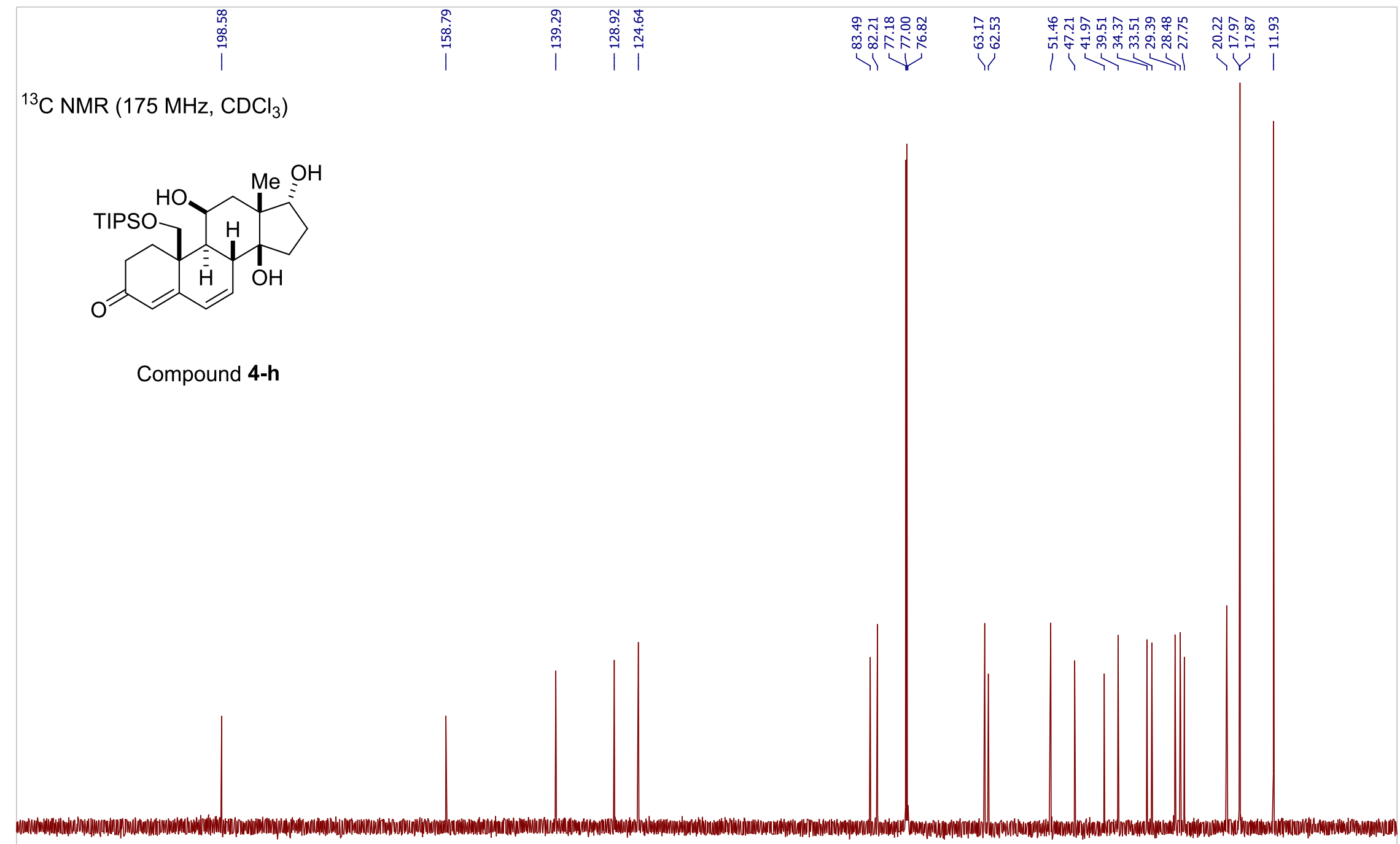




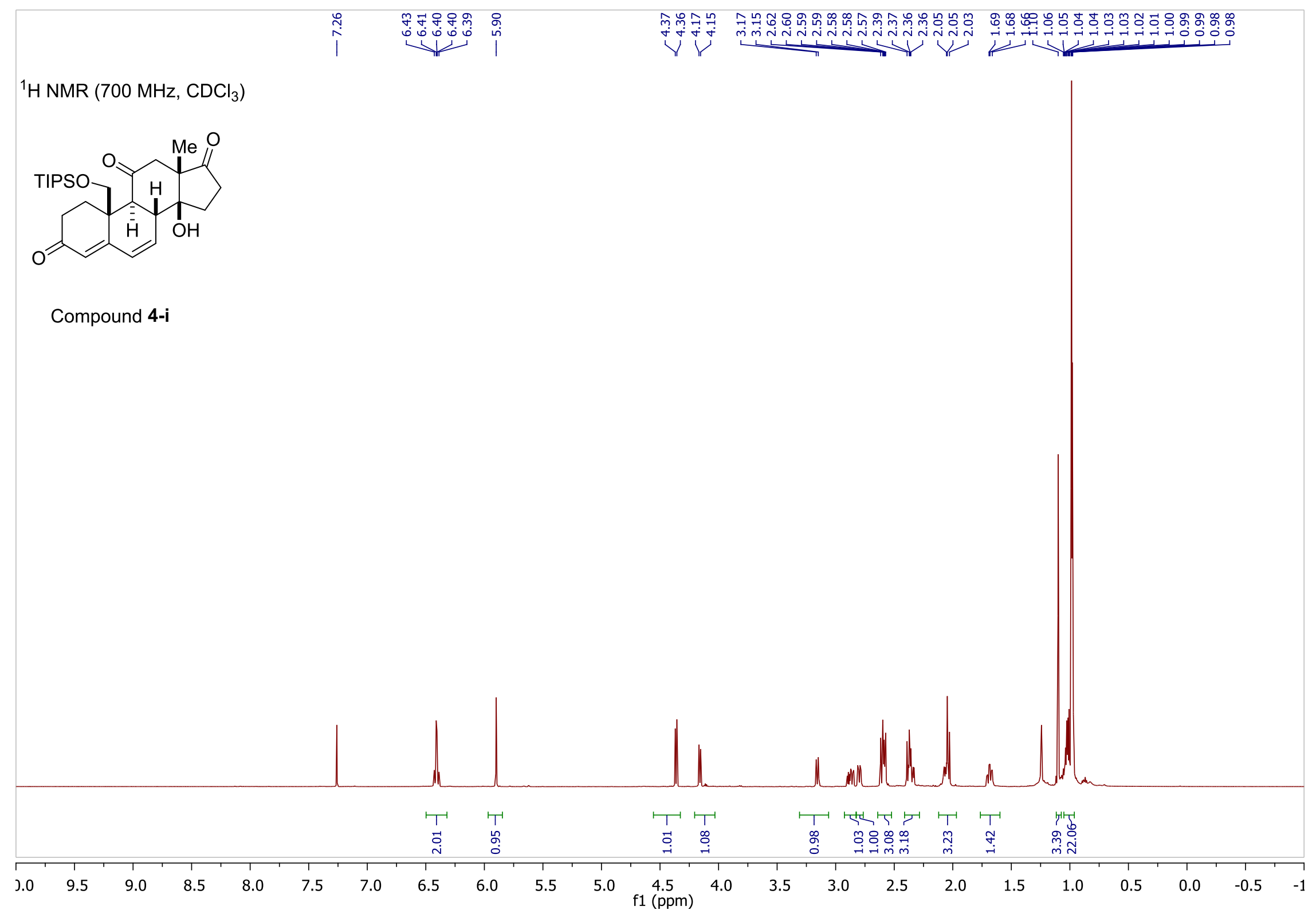




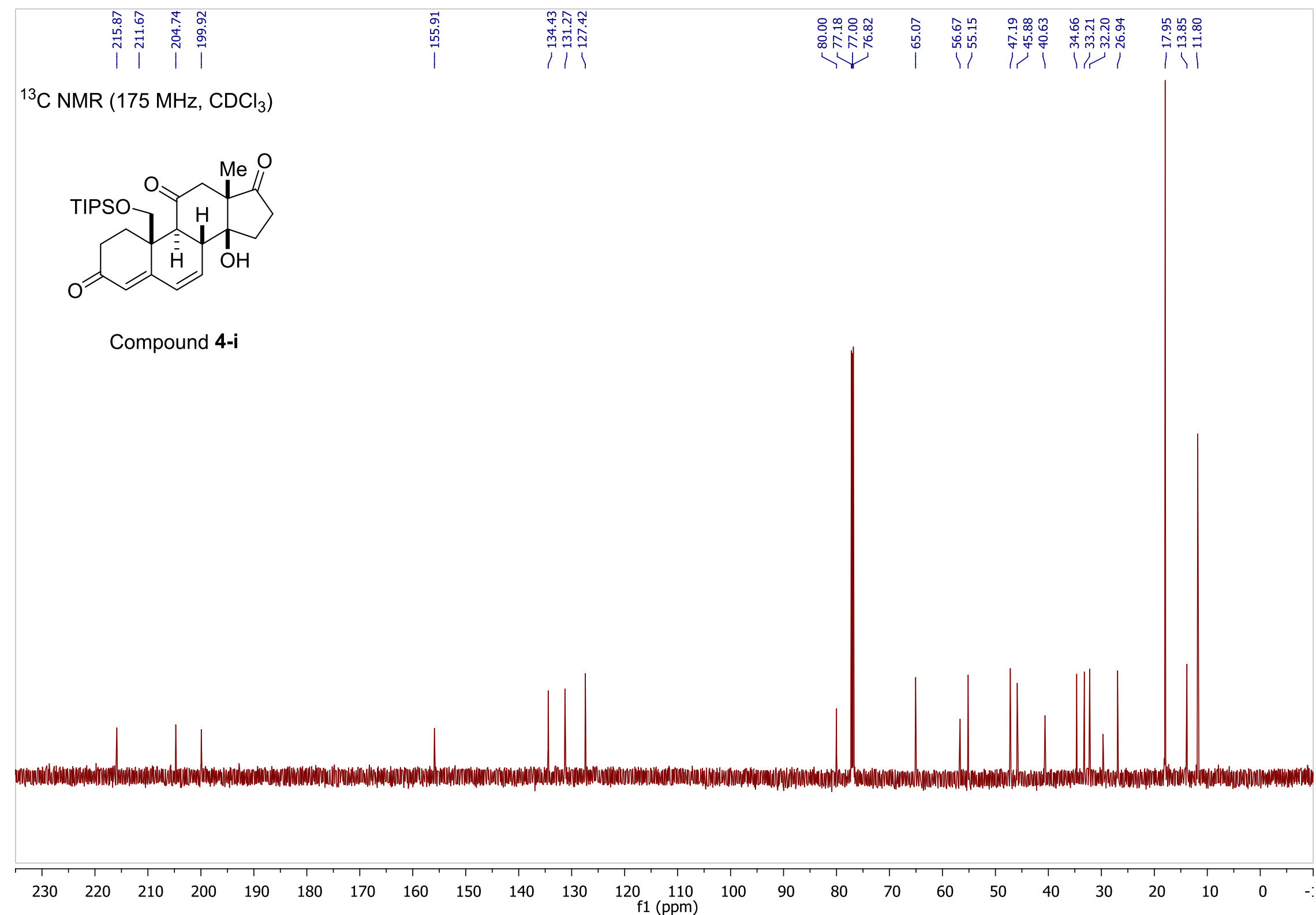


${ }^{1} \mathrm{H}$ NMR $\left(700 \mathrm{MHz}, \mathrm{CDCl}_{3}\right)$

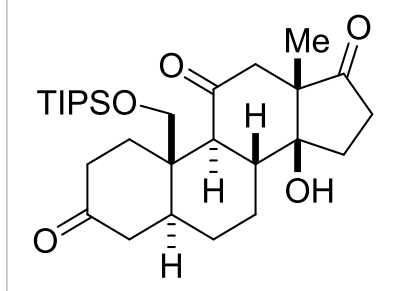

Compound 18

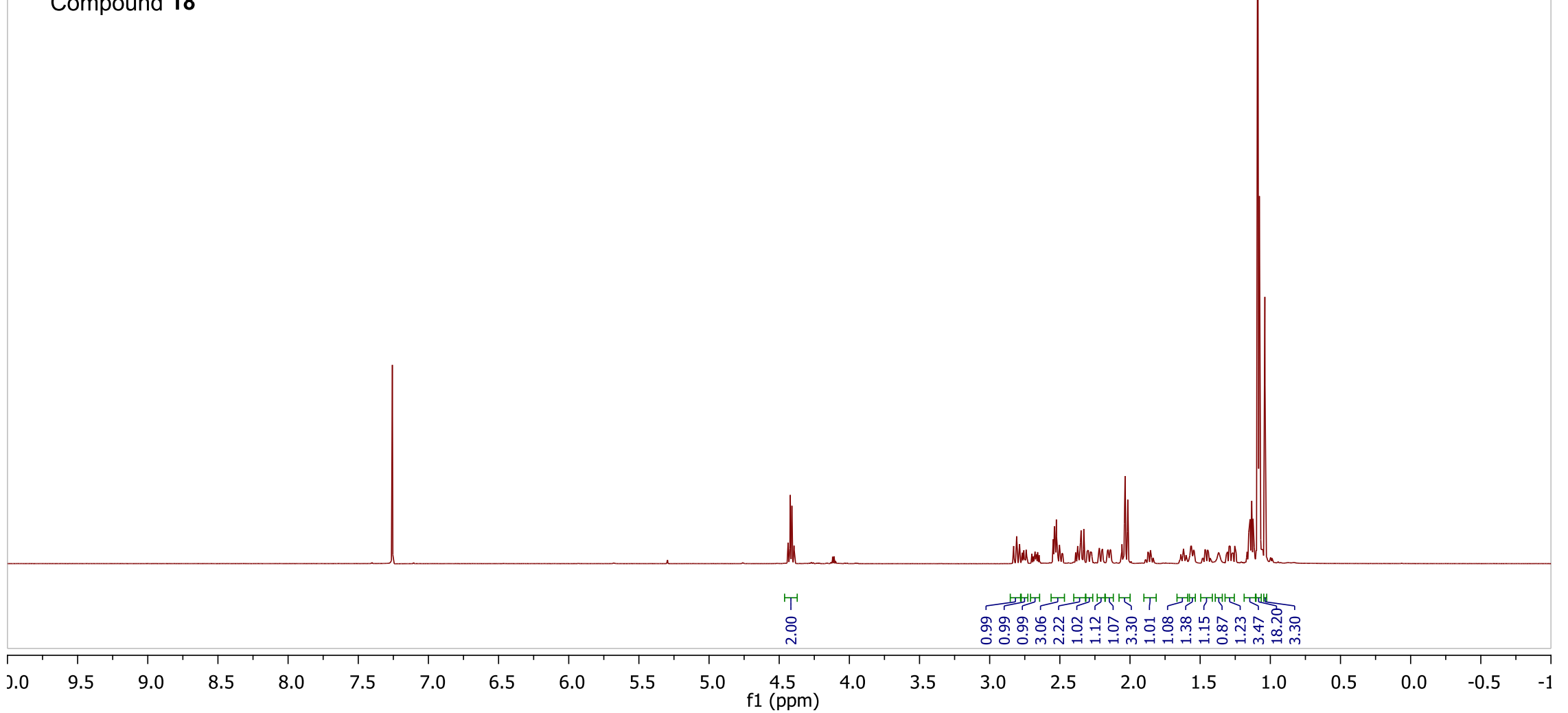




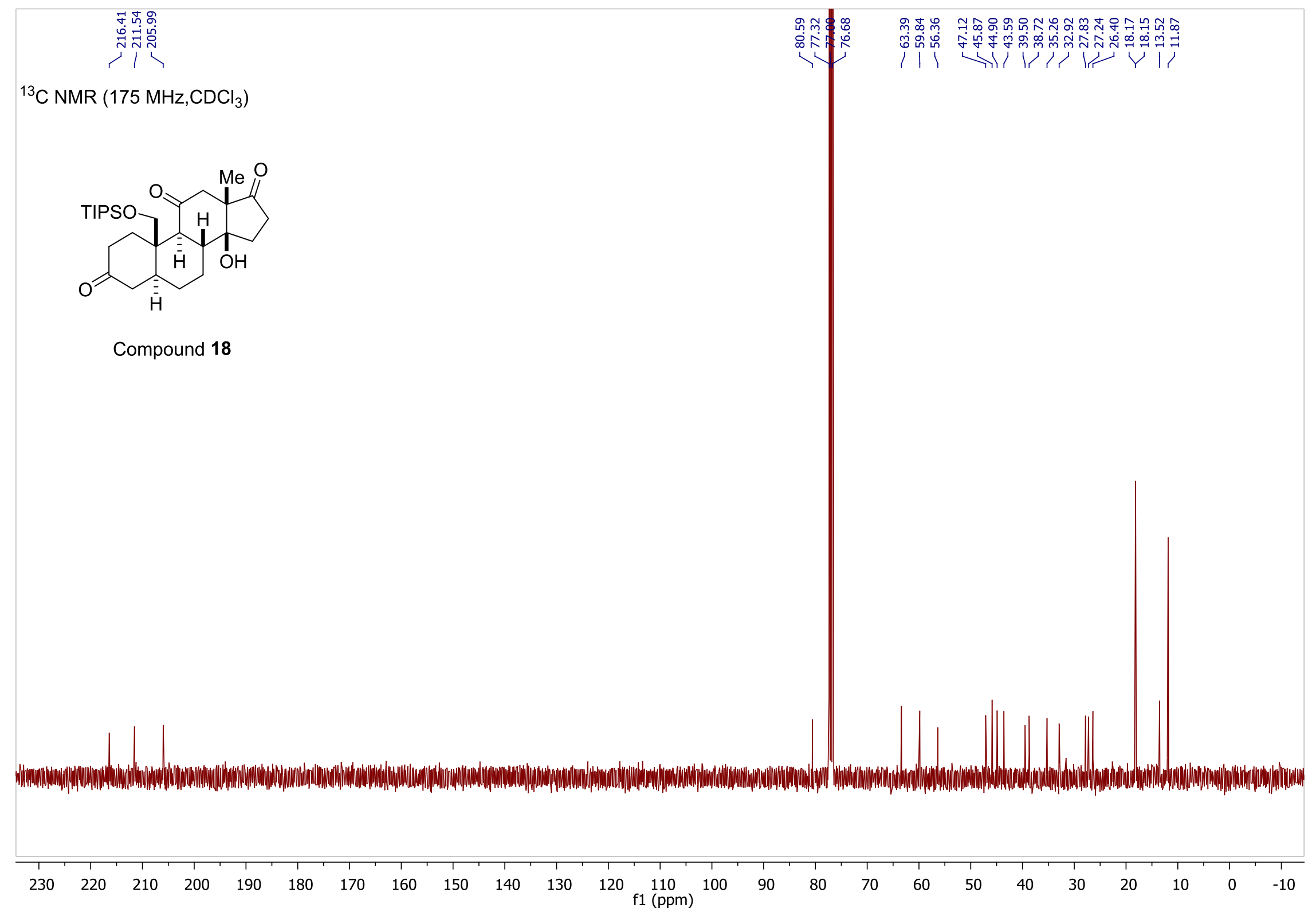




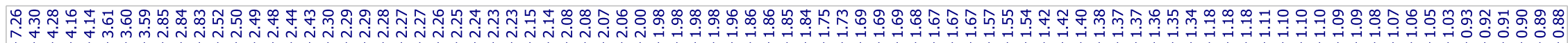

${ }^{1} \mathrm{H} \mathrm{NMR}\left(700 \mathrm{MHz}, \mathrm{CDCl}_{3}\right)$

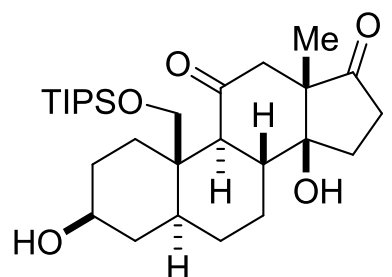

Compound 18-j

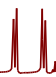

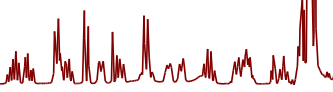

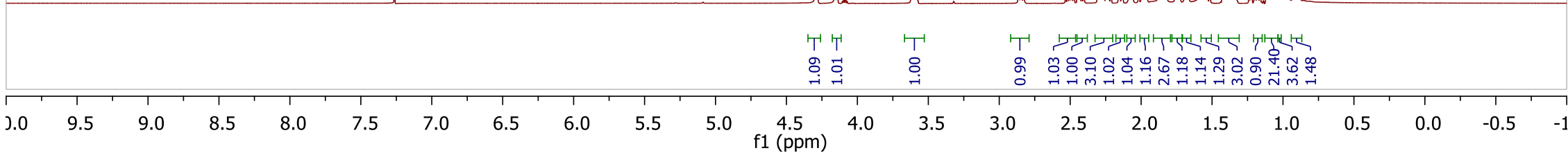




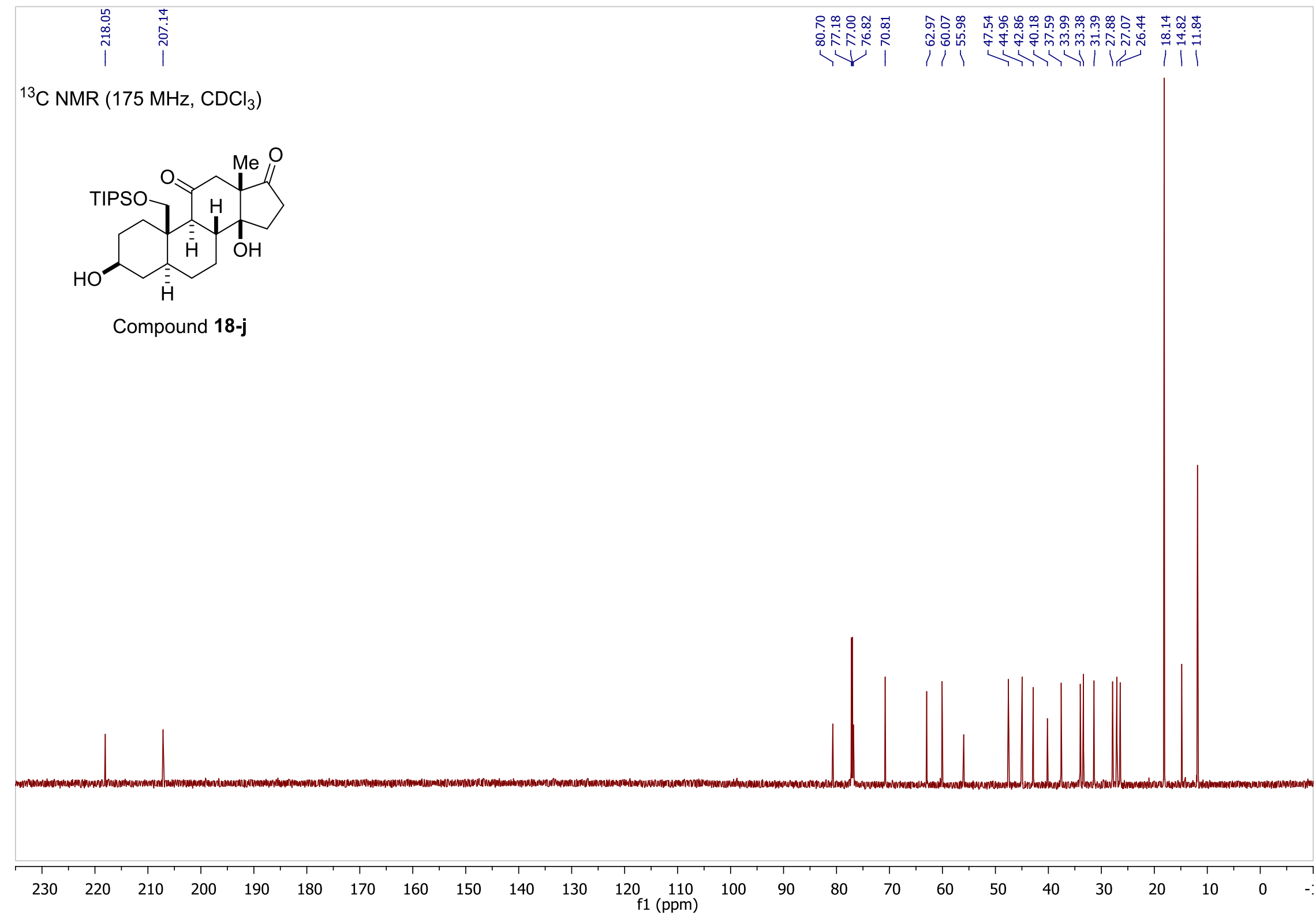


$\operatorname{cosY}\left(175 \mathrm{MHz}, \mathrm{CDCl}_{3}\right)$

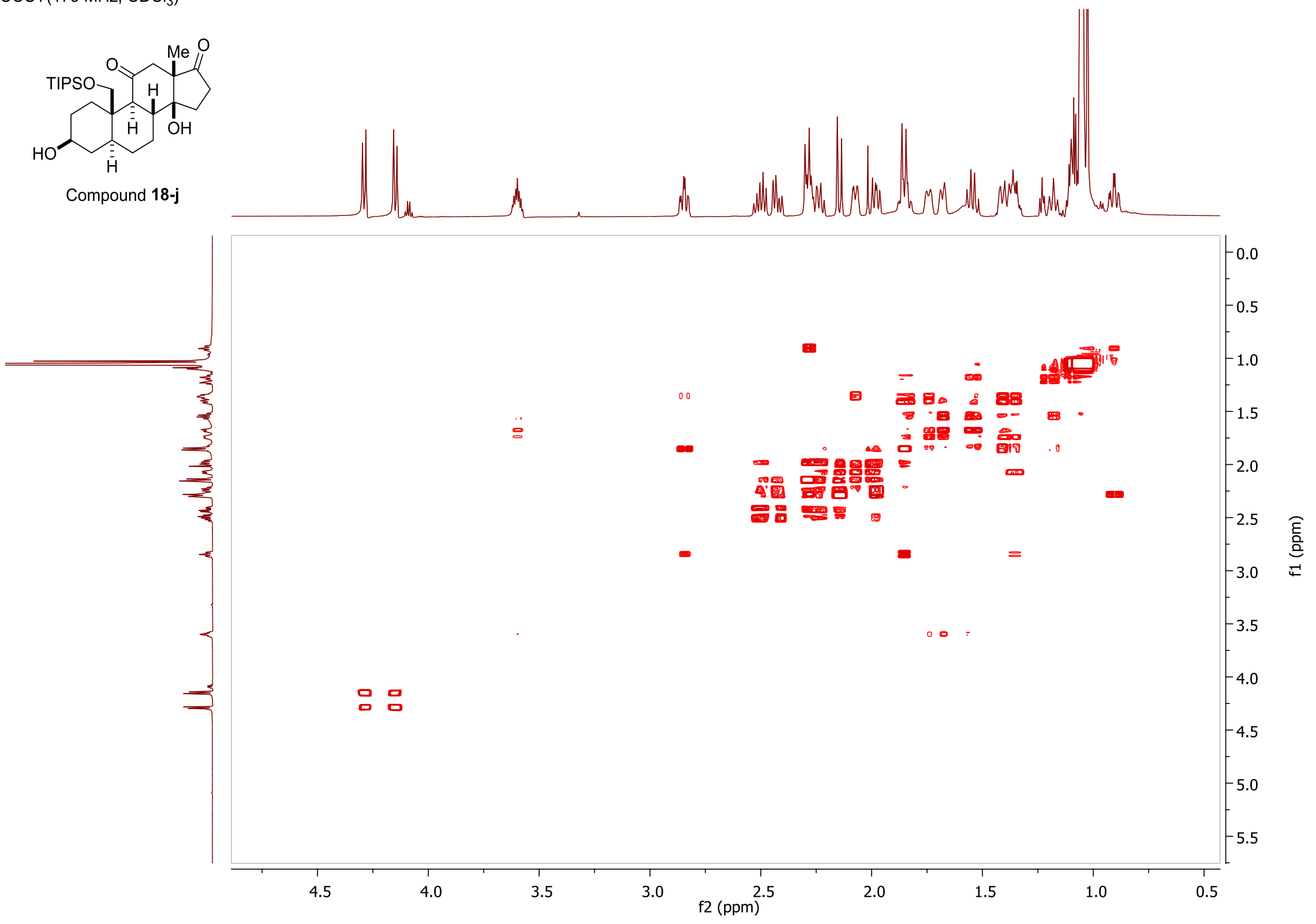


HSQC (175 MHz, $\mathrm{CDCl}_{3}$ )

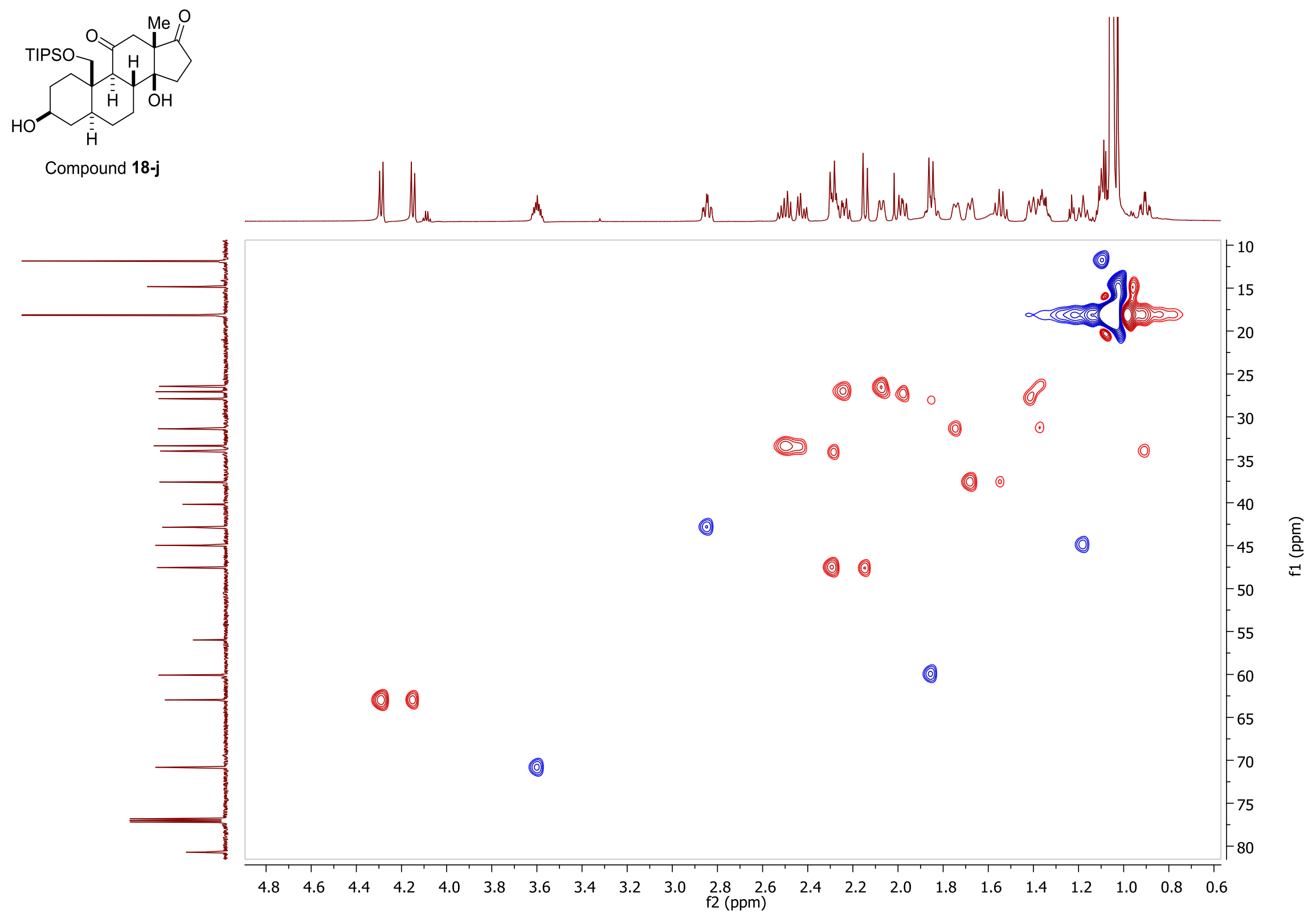




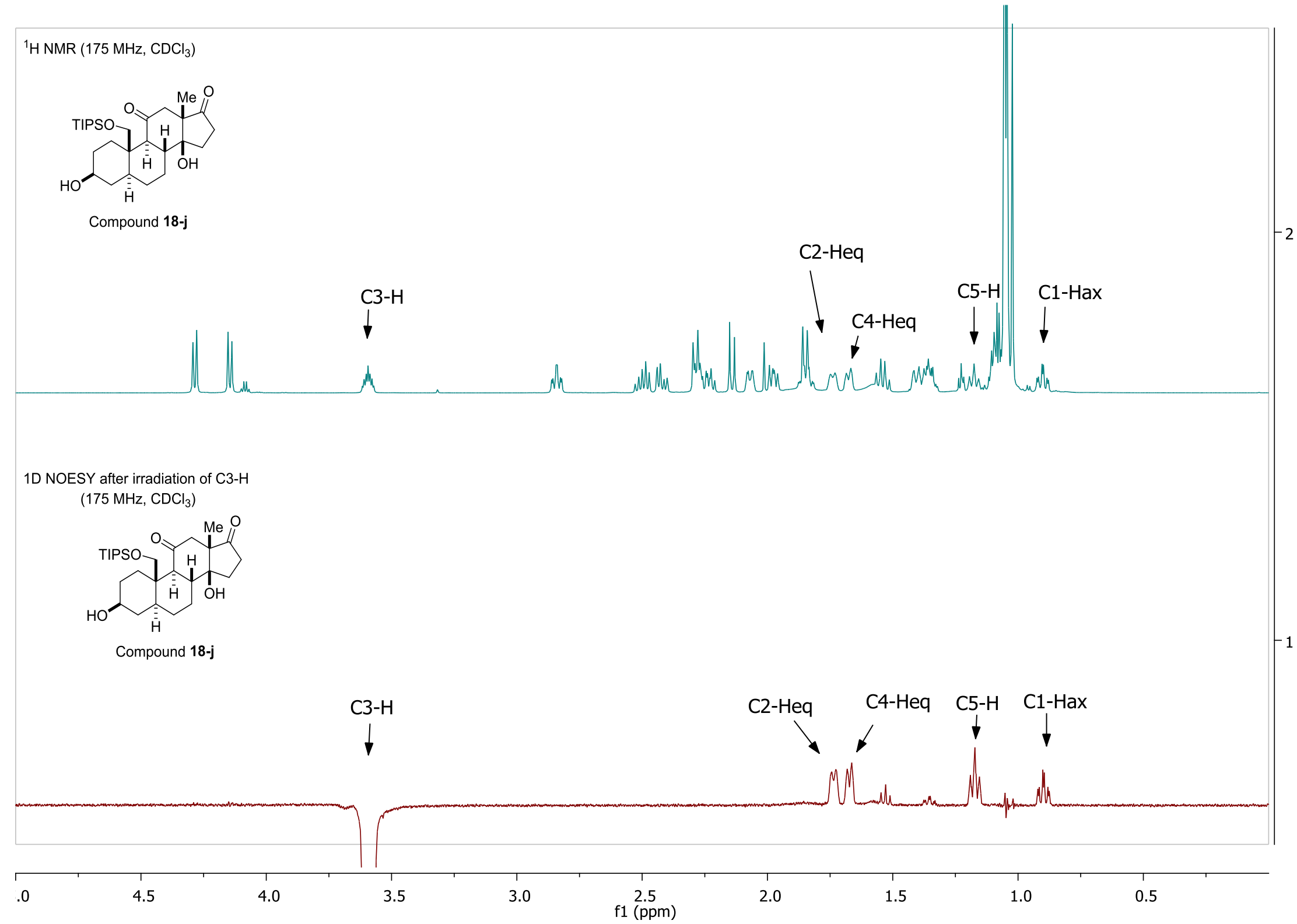


${ }^{1} \mathrm{H}$ NMR $\left(700 \mathrm{MHz}, \mathrm{C}_{6} \mathrm{D}_{6}\right)$

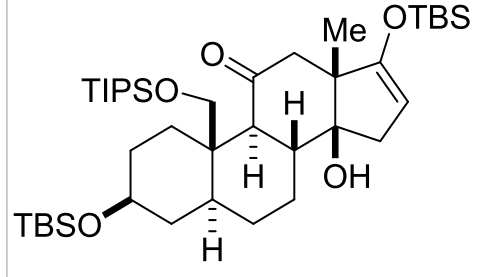

Compound 23

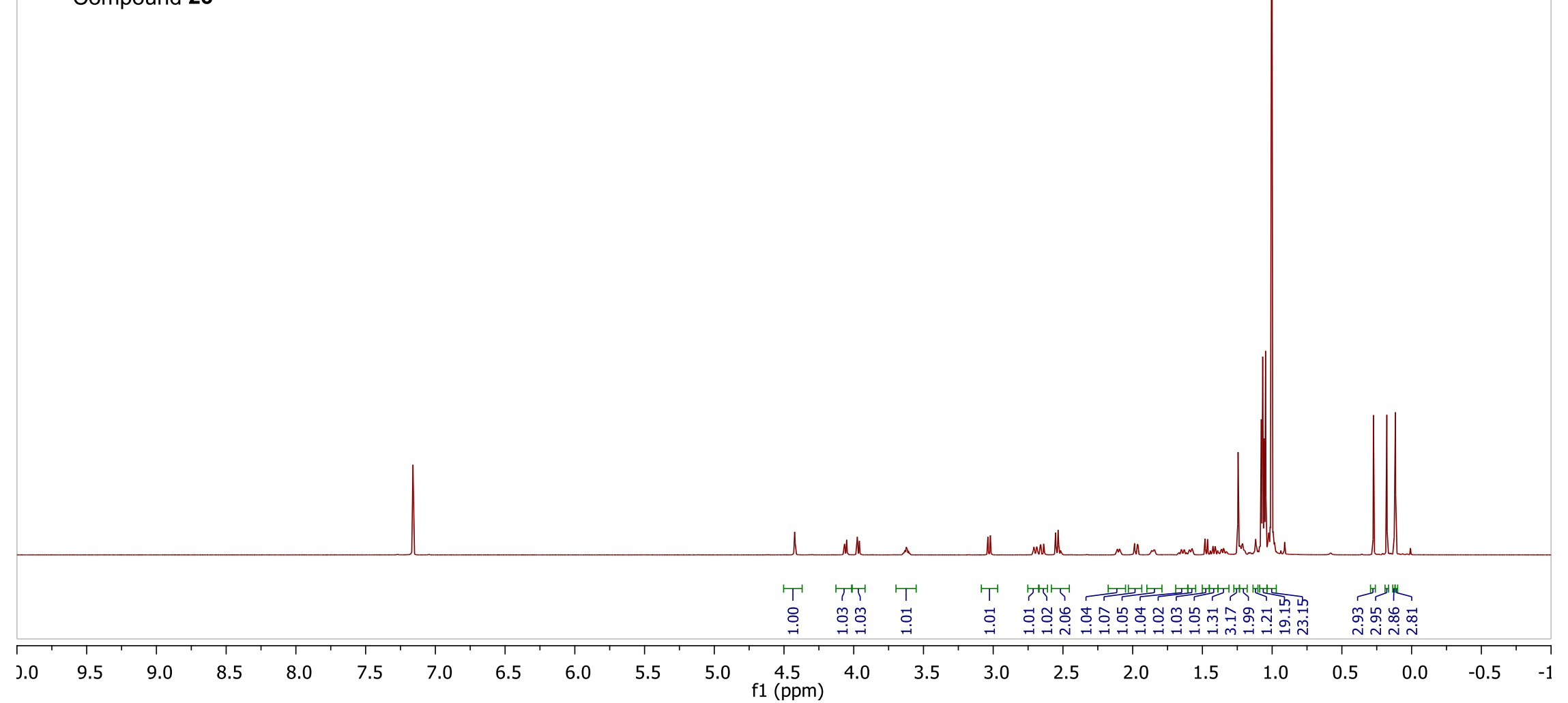




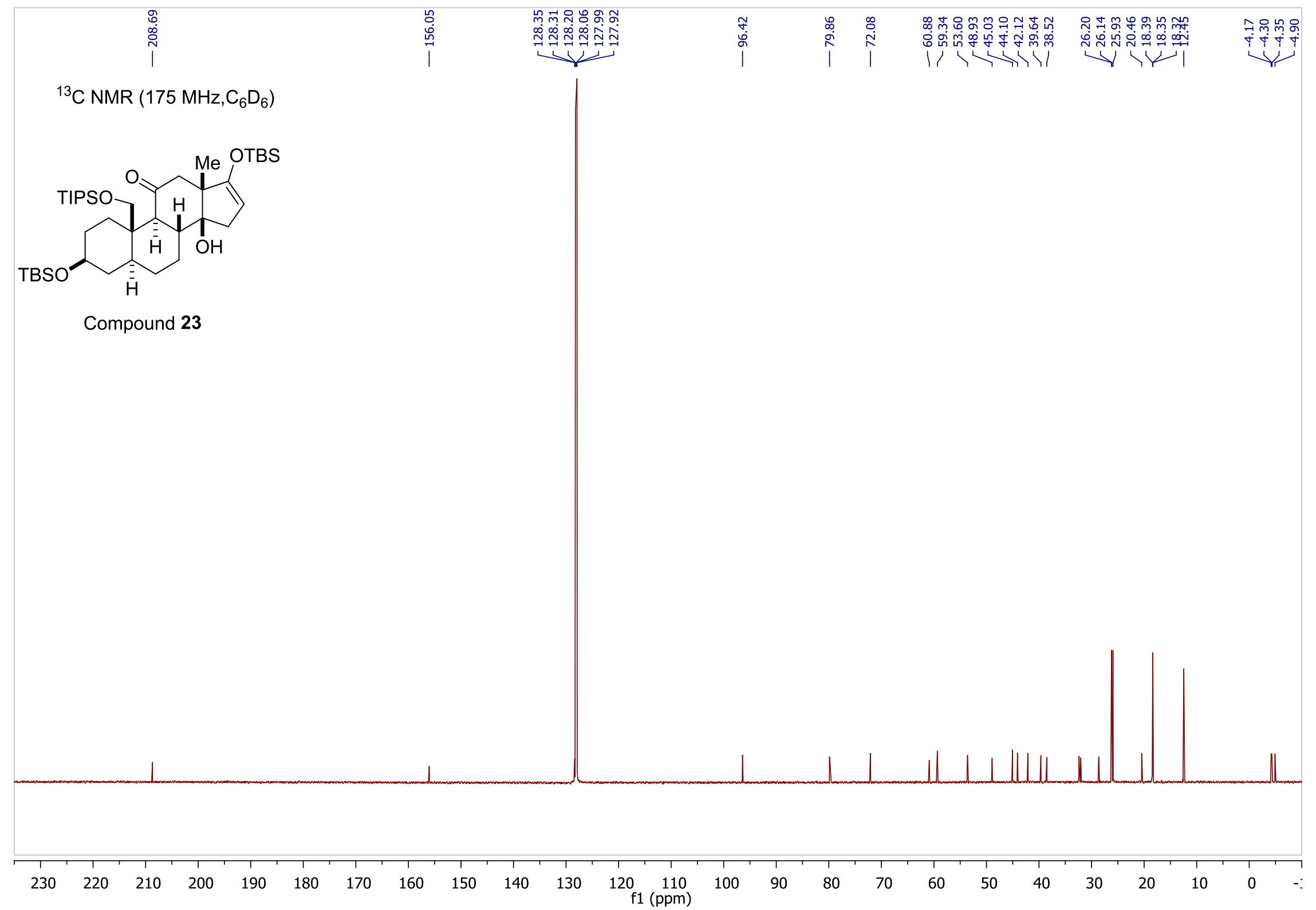


${ }^{1} \mathrm{H}$ NMR $\left(700 \mathrm{MHz}, \mathrm{CDCl}_{3}\right)$

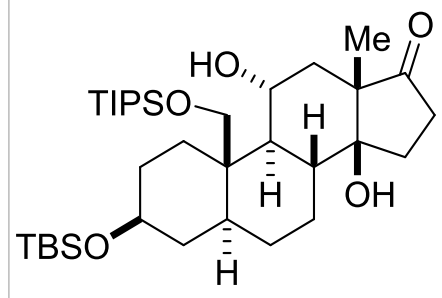

Compound 23-m

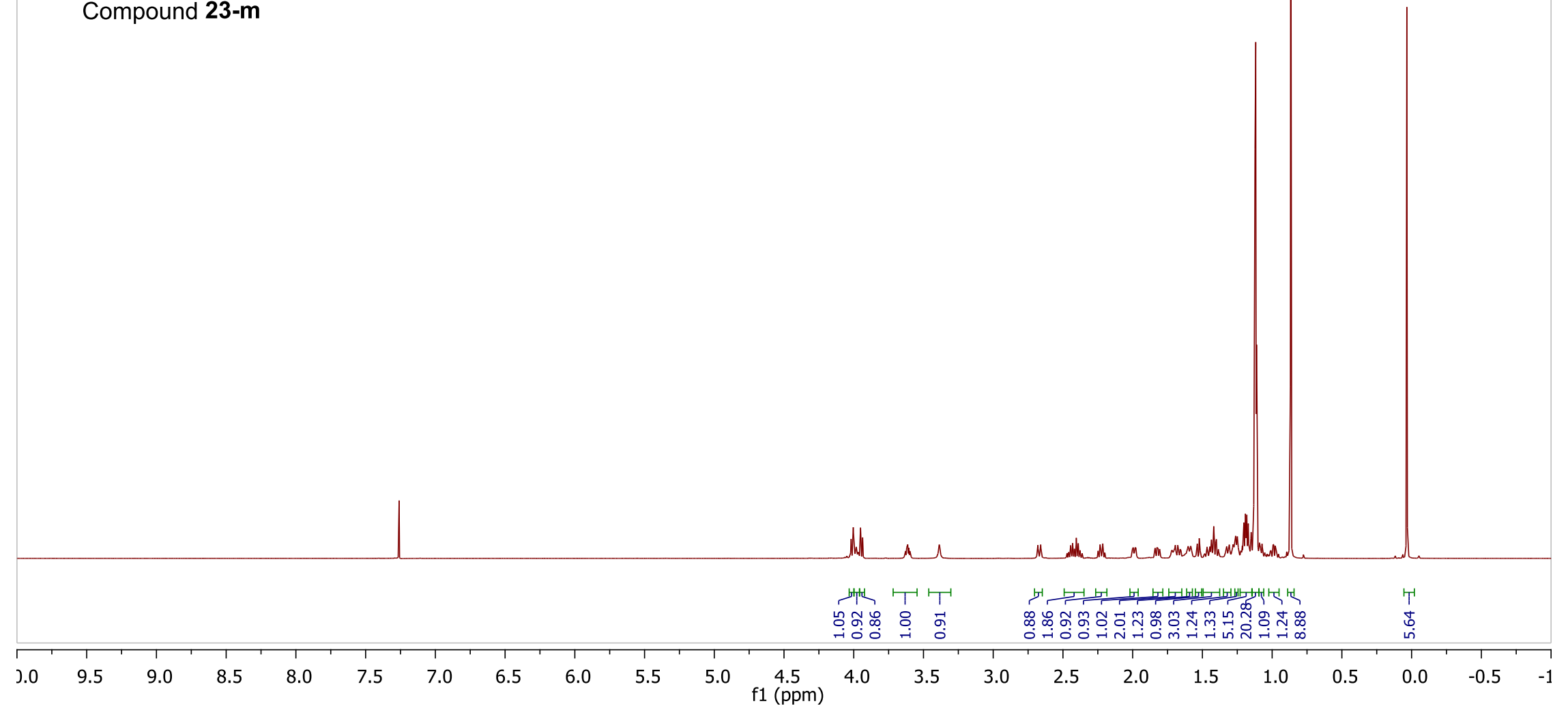




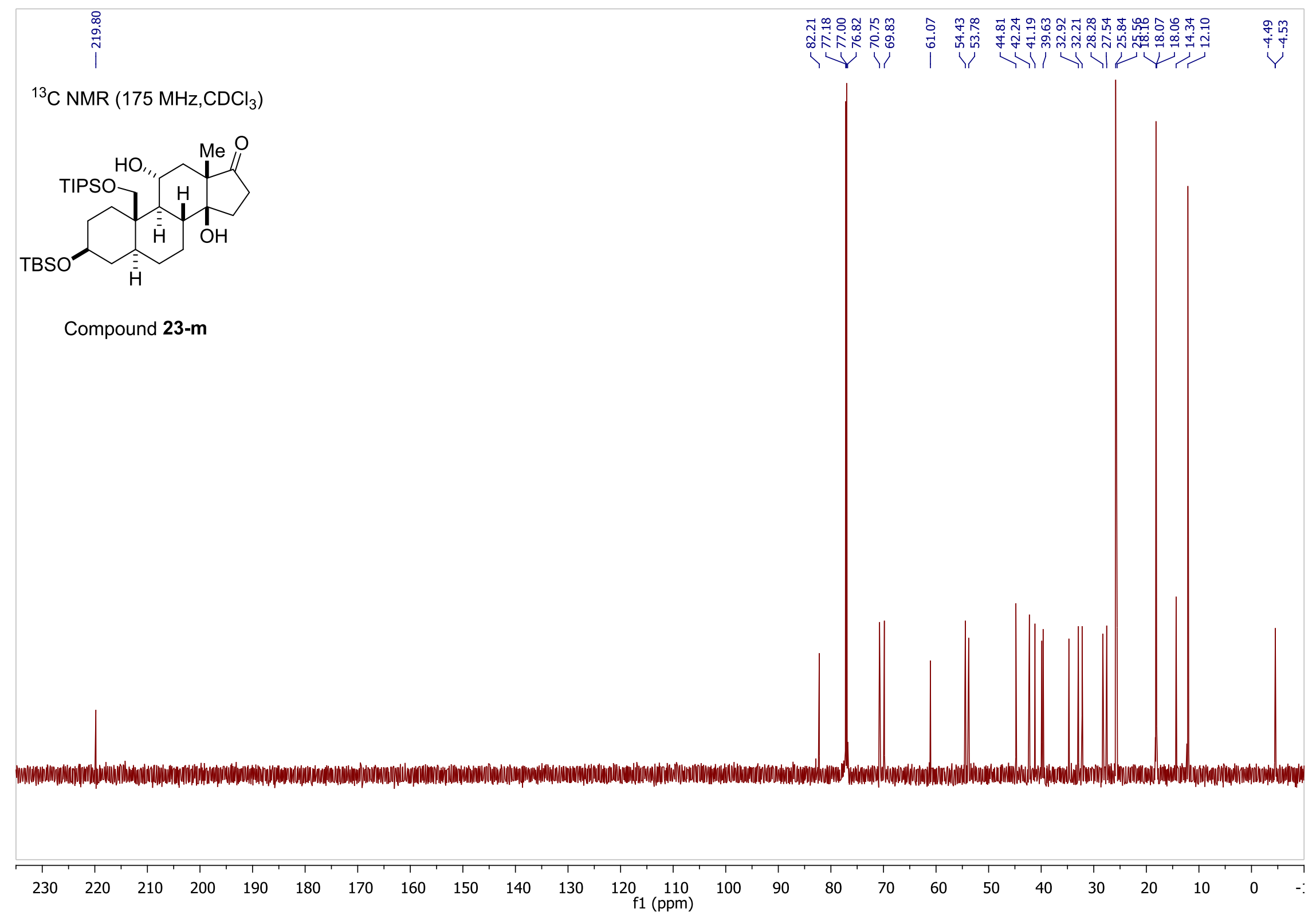


${ }^{1} \mathrm{H}$ NMR $\left(700 \mathrm{MHz}, \mathrm{CDCl}_{3}\right)$

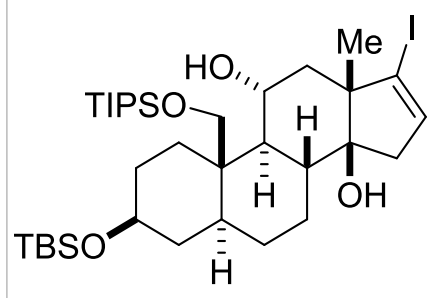

Compound 24

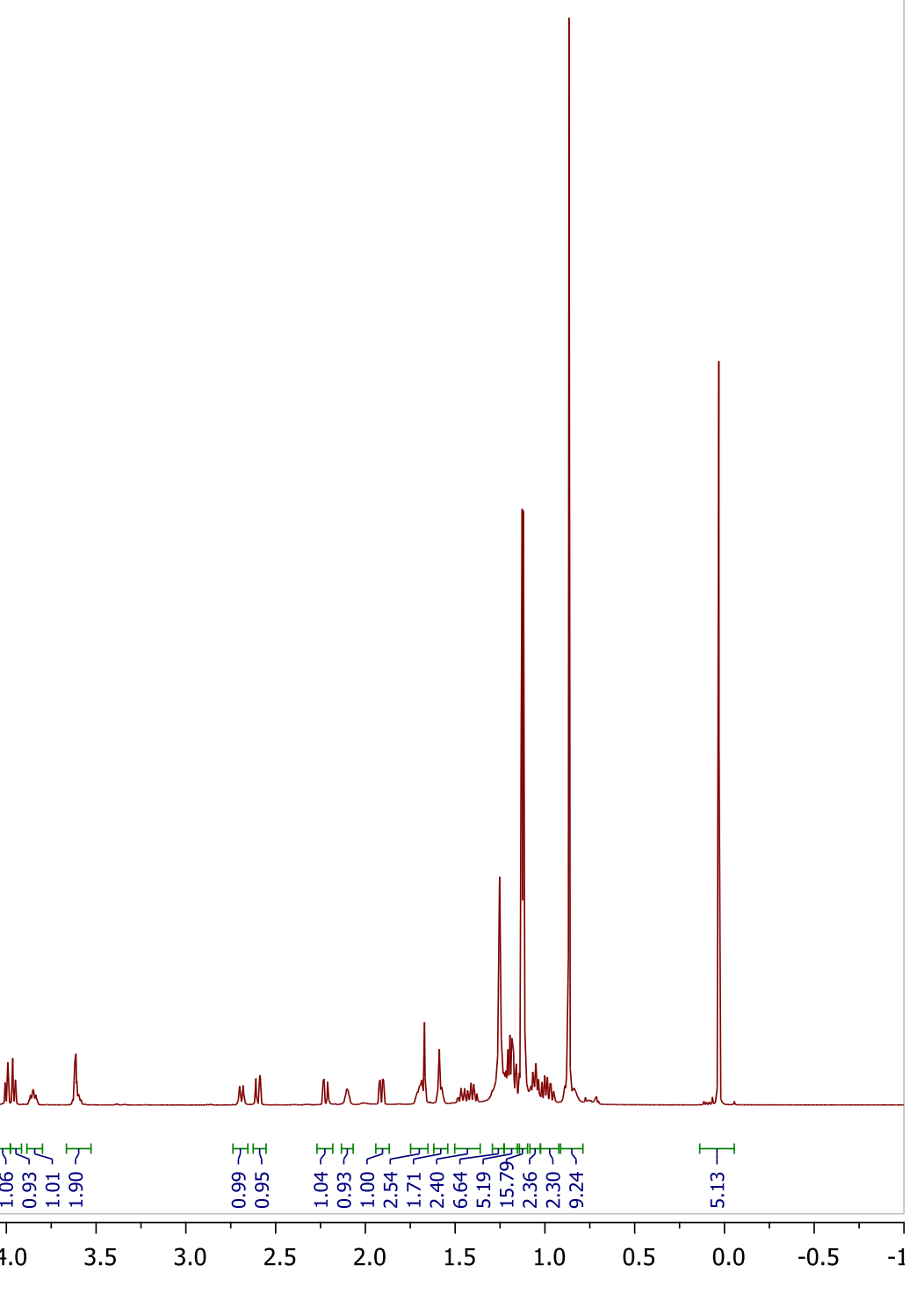




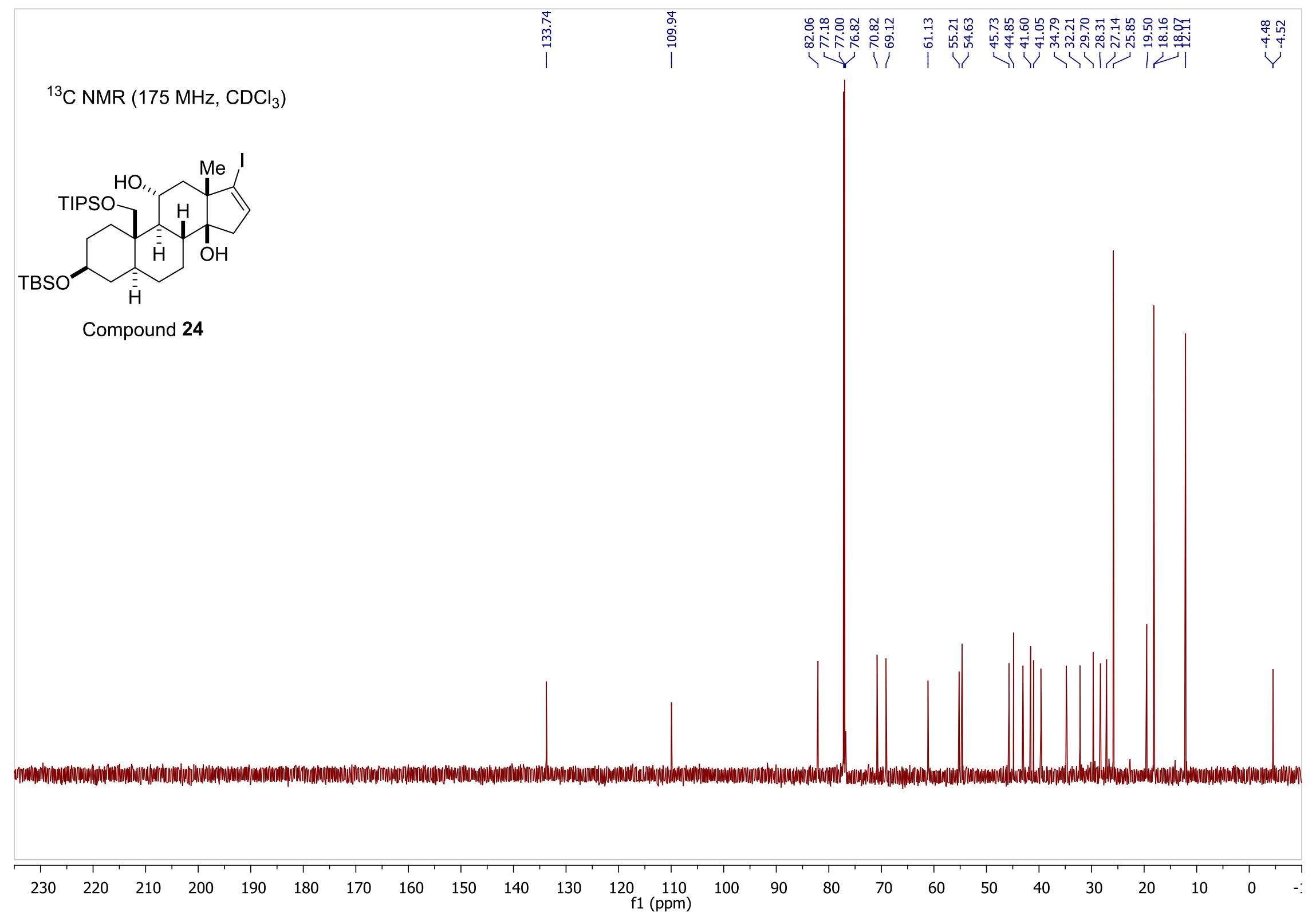




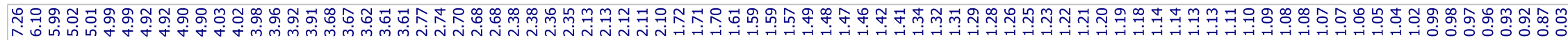

${ }^{1} \mathrm{H}$ NMR $\left(700 \mathrm{MHz}, \mathrm{CDCl}_{3}\right)$

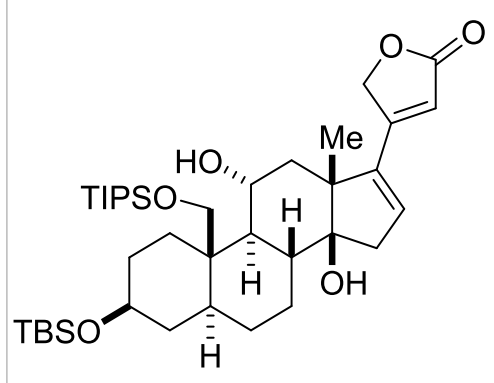

Compound 25

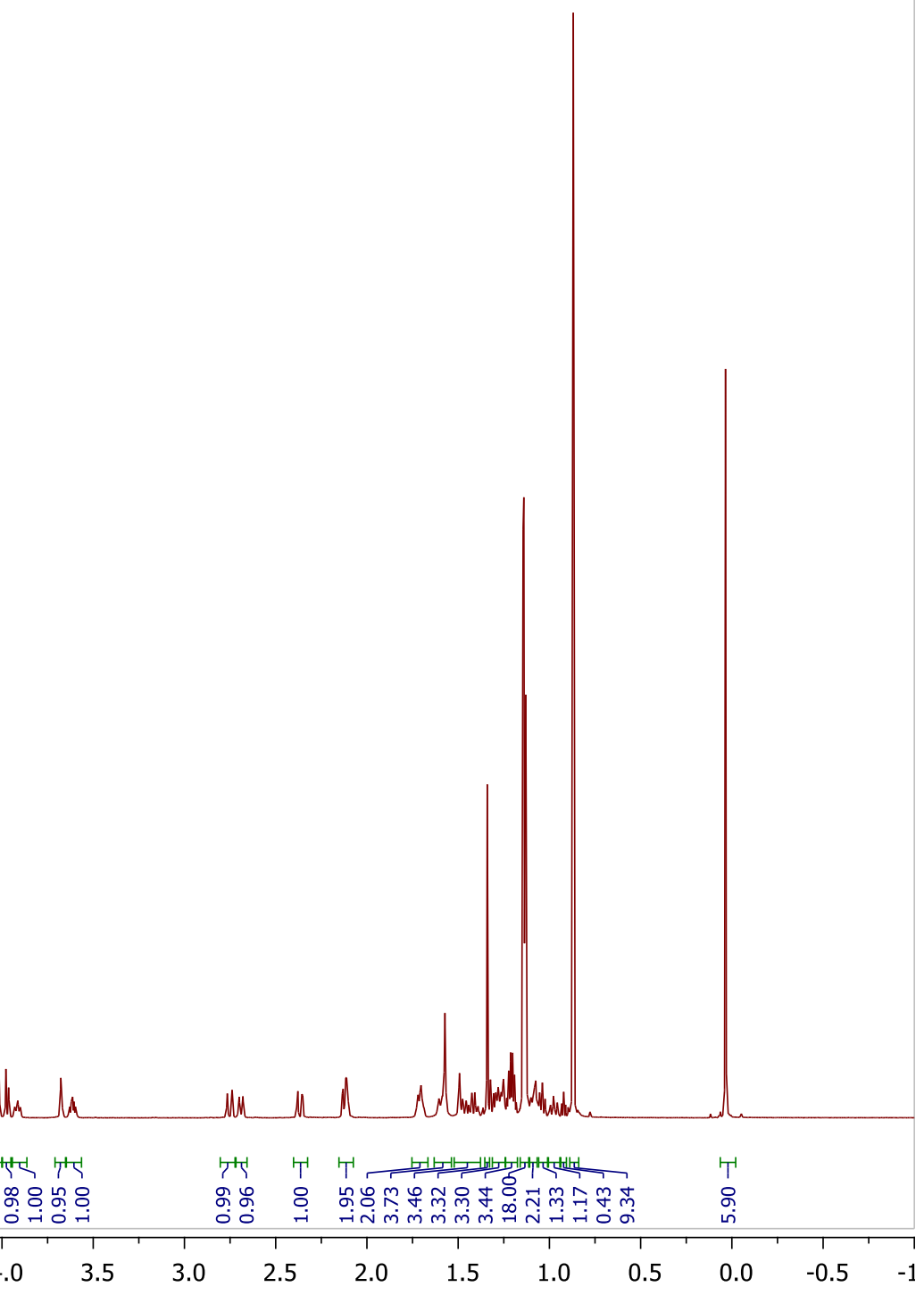




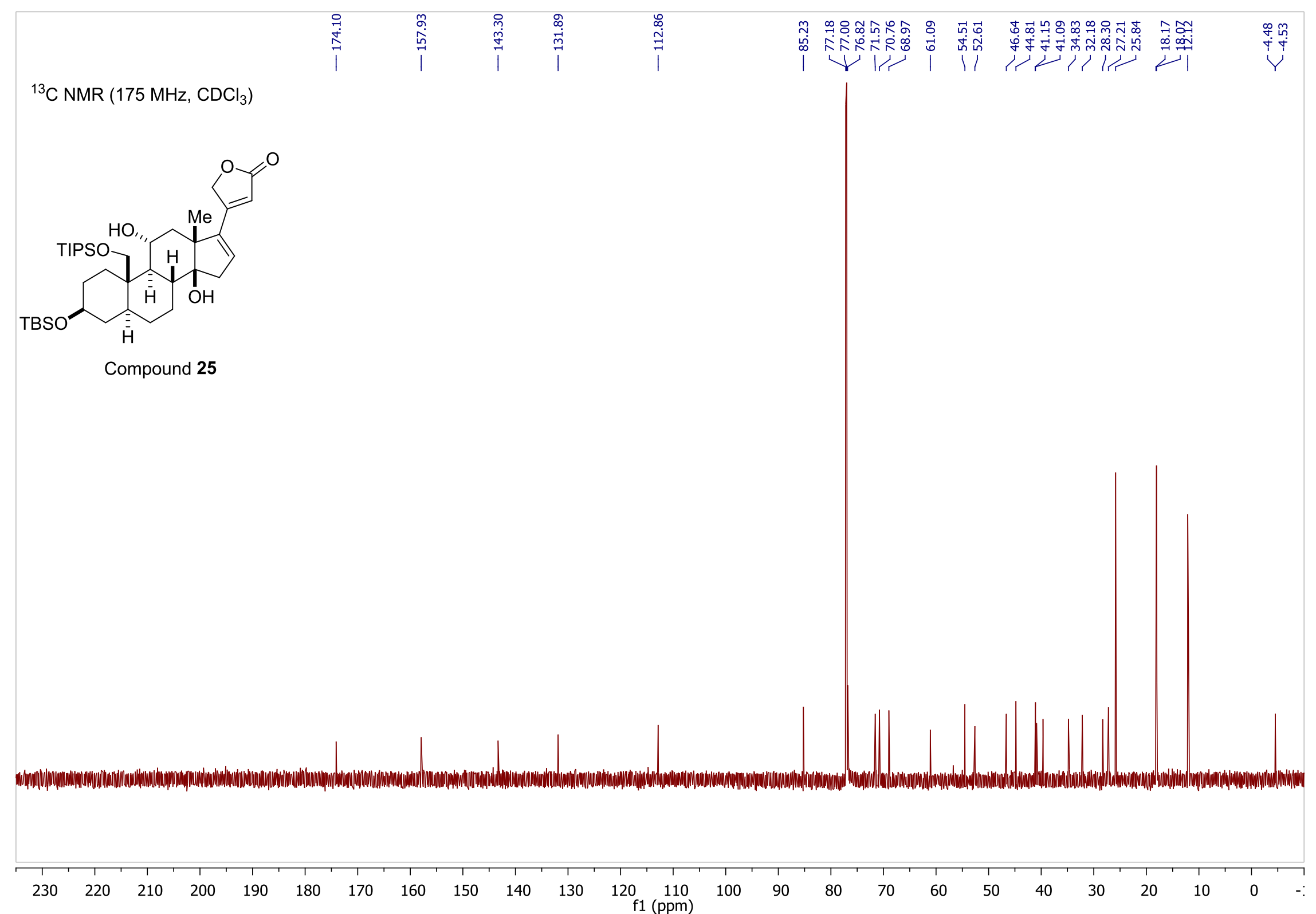




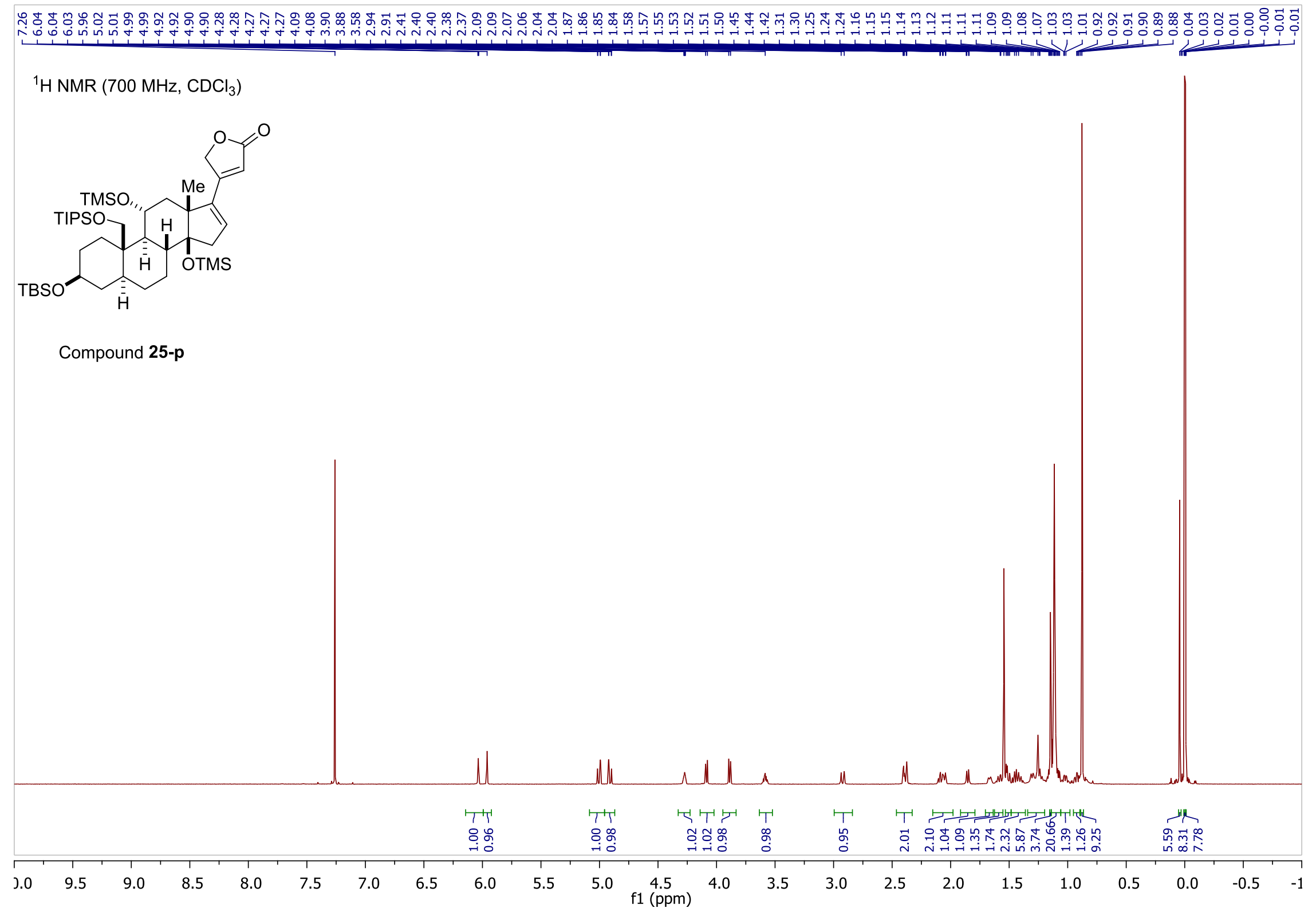




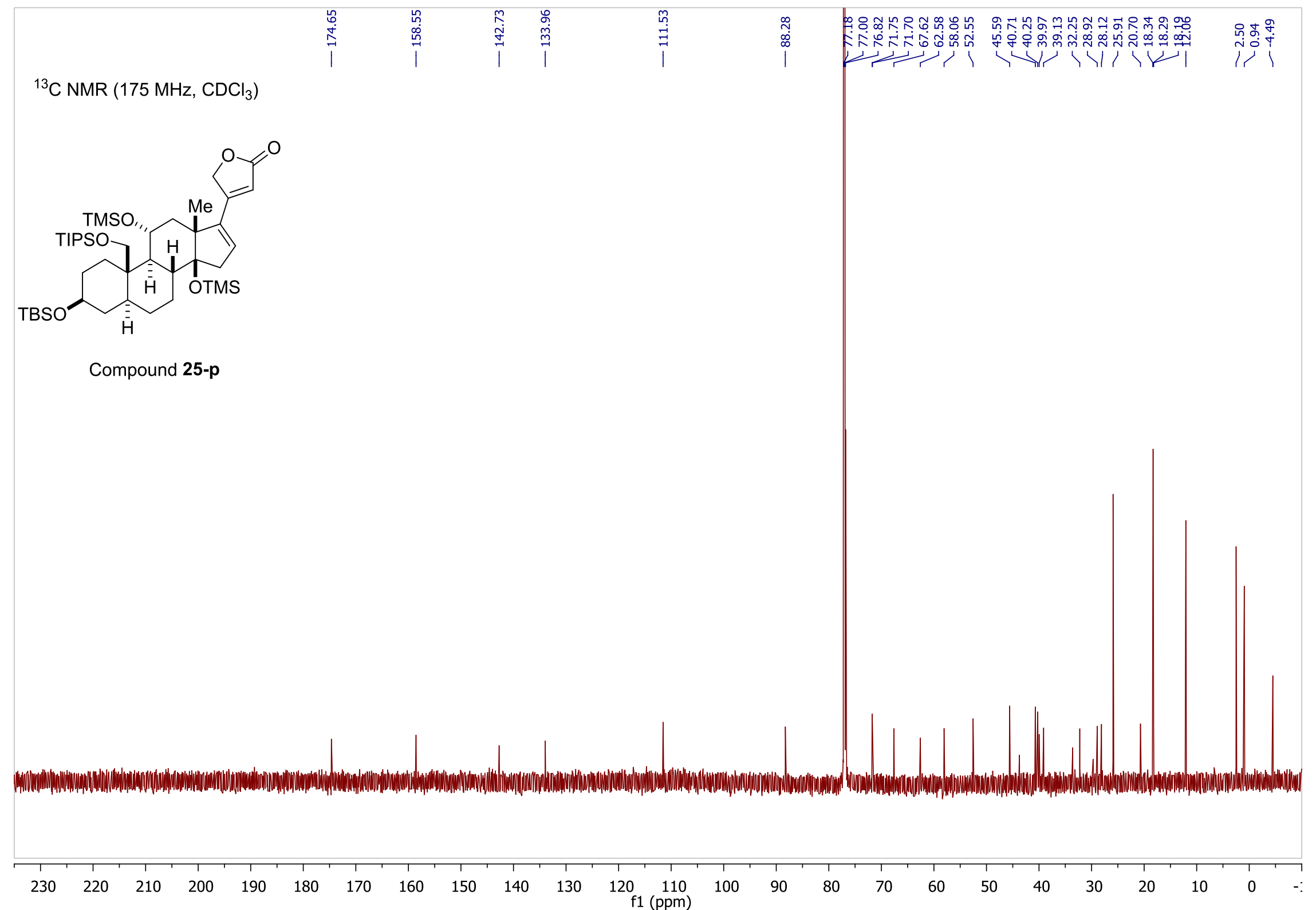




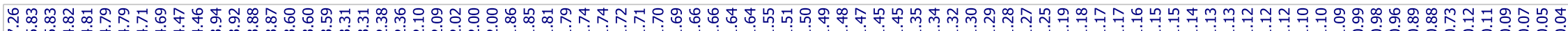

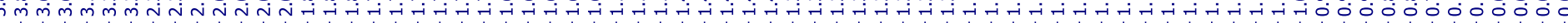

${ }^{1} \mathrm{H}$ NMR $\left(700 \mathrm{MHz}, \mathrm{CDCl}_{3}\right)$

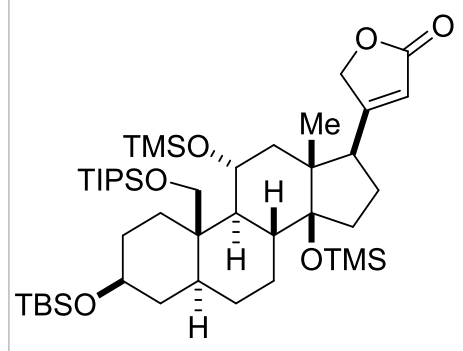

Compound 25-q

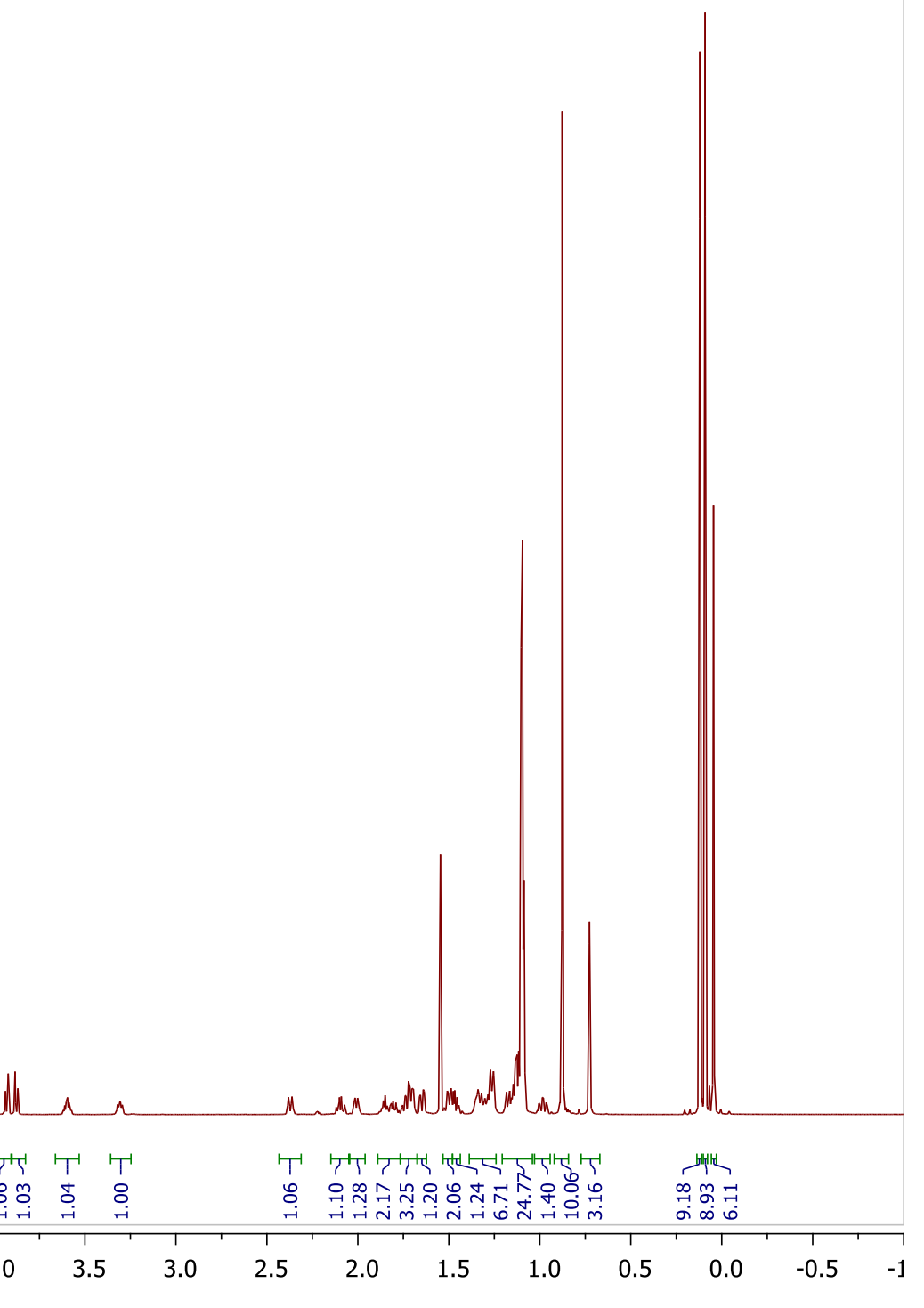




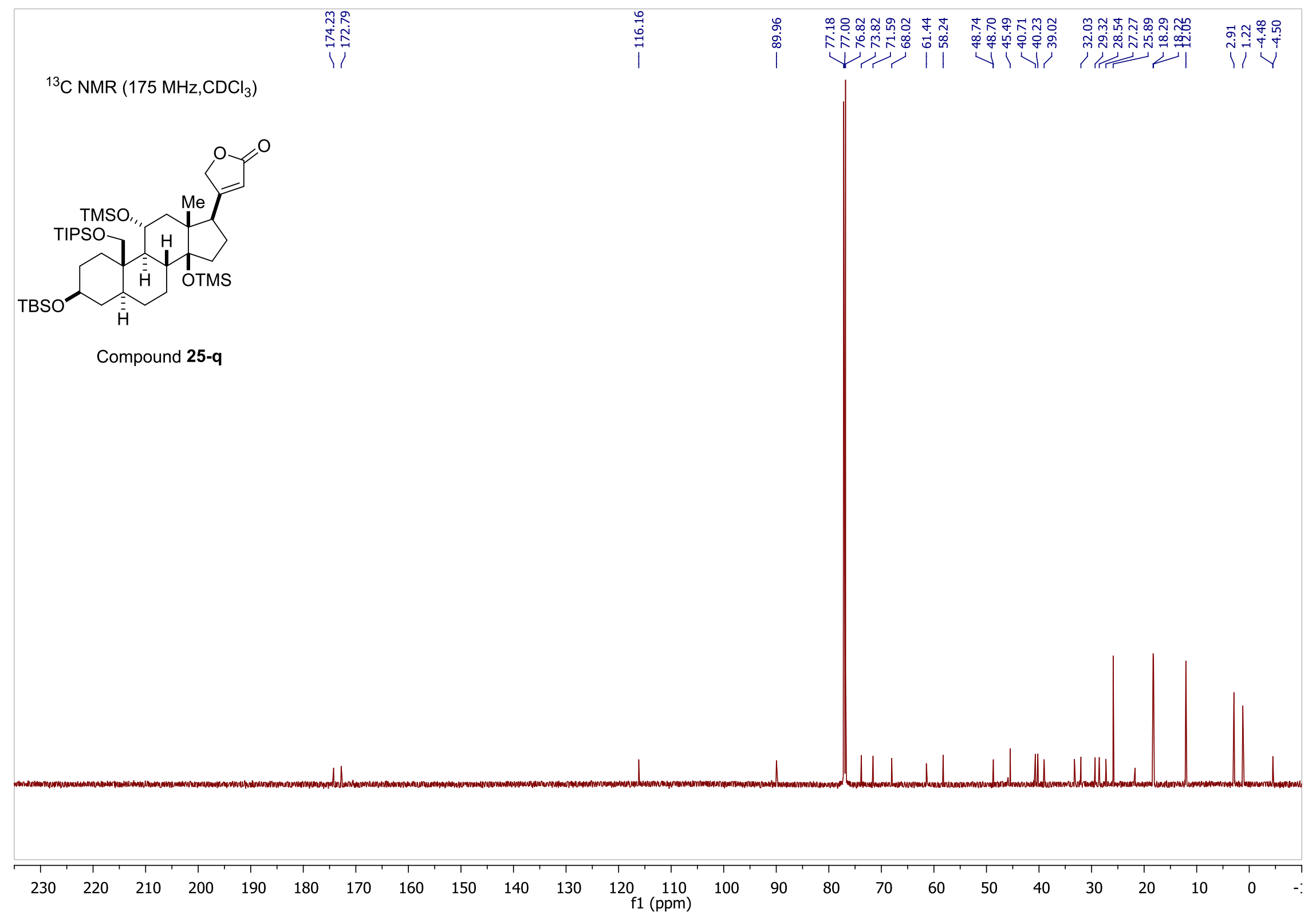


$\operatorname{cosY}\left(175 \mathrm{MHz}, \mathrm{CDCl}_{3}\right)$

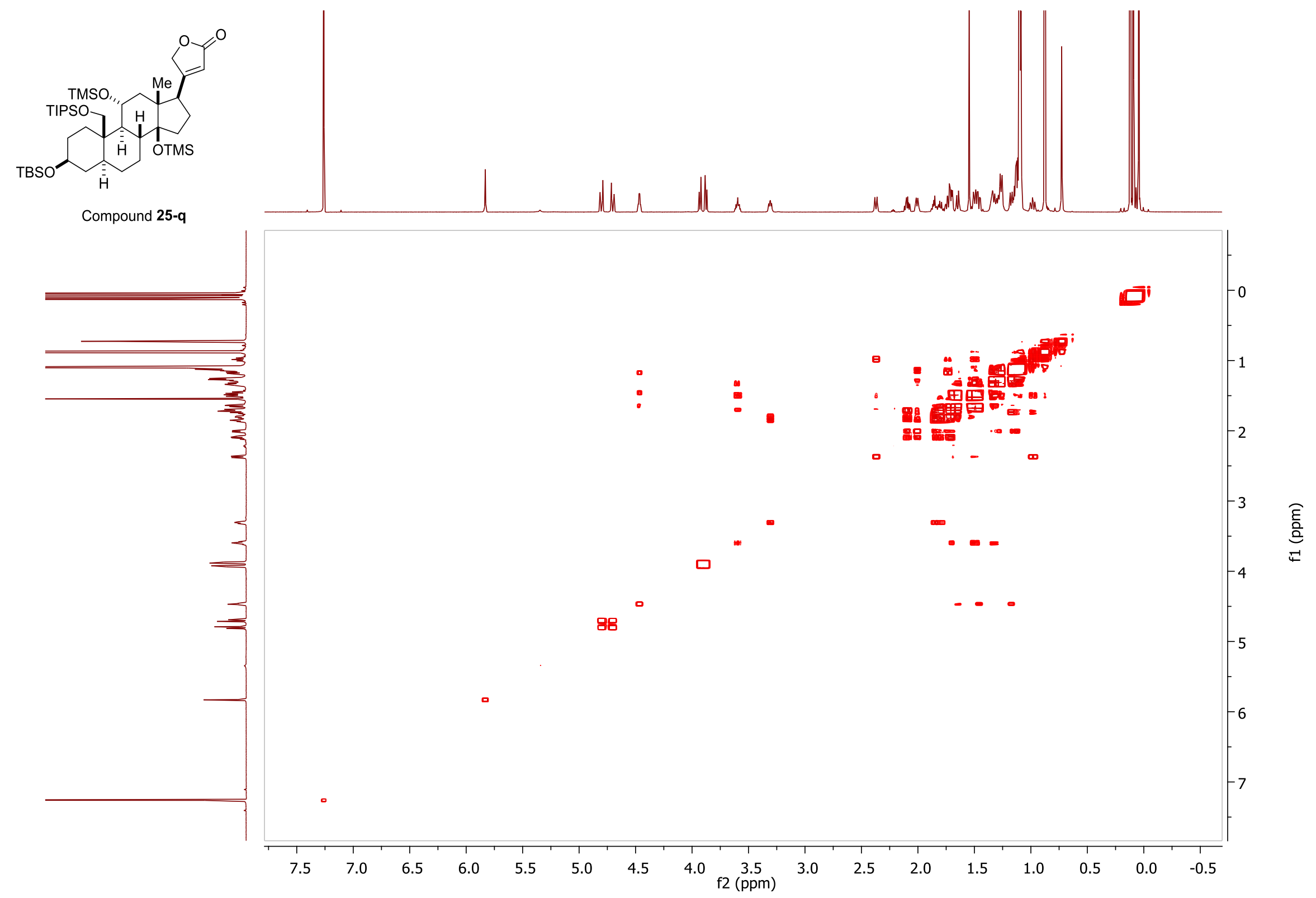


HSQC (175 MHz,CDCl $\left.{ }_{3}\right)$

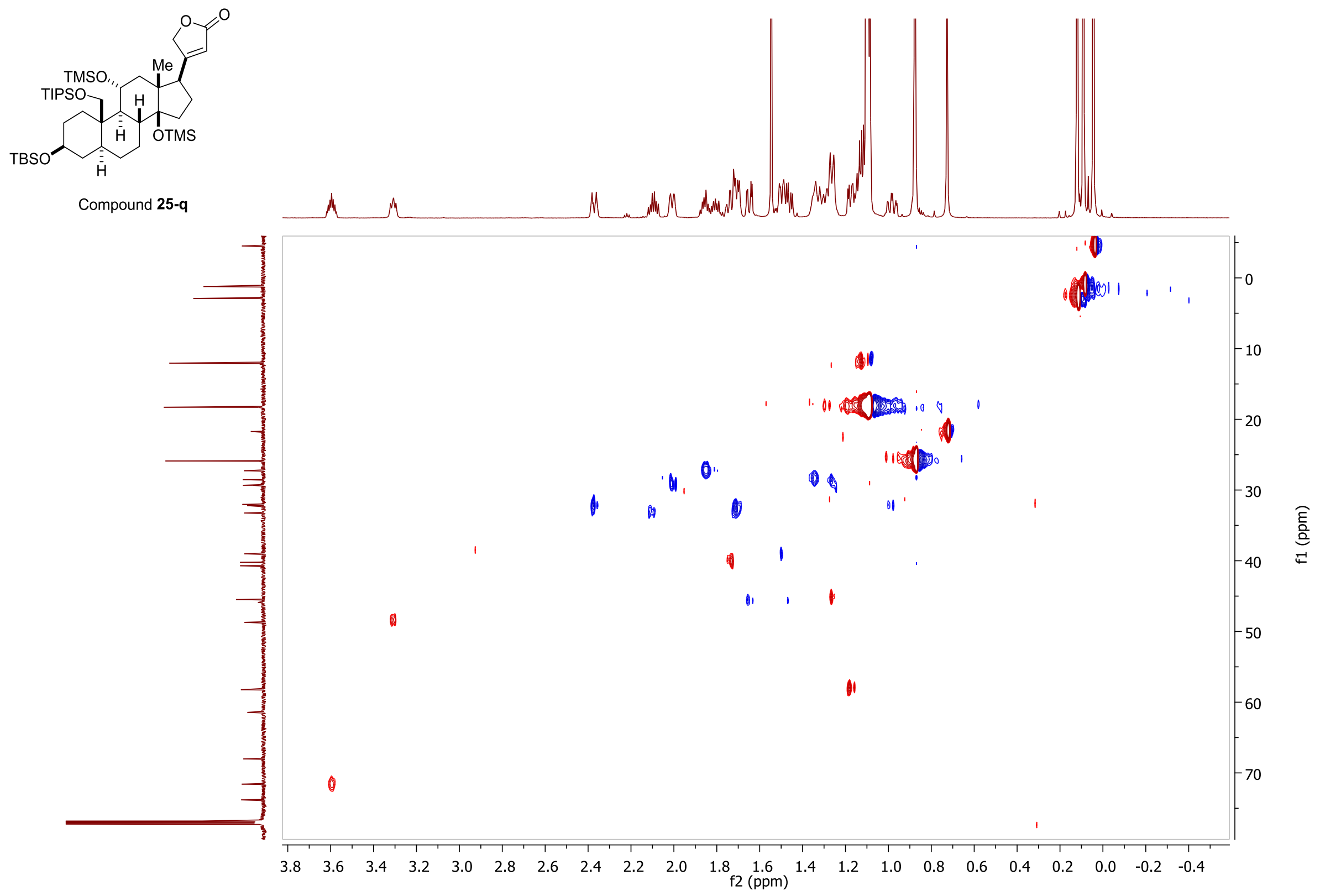




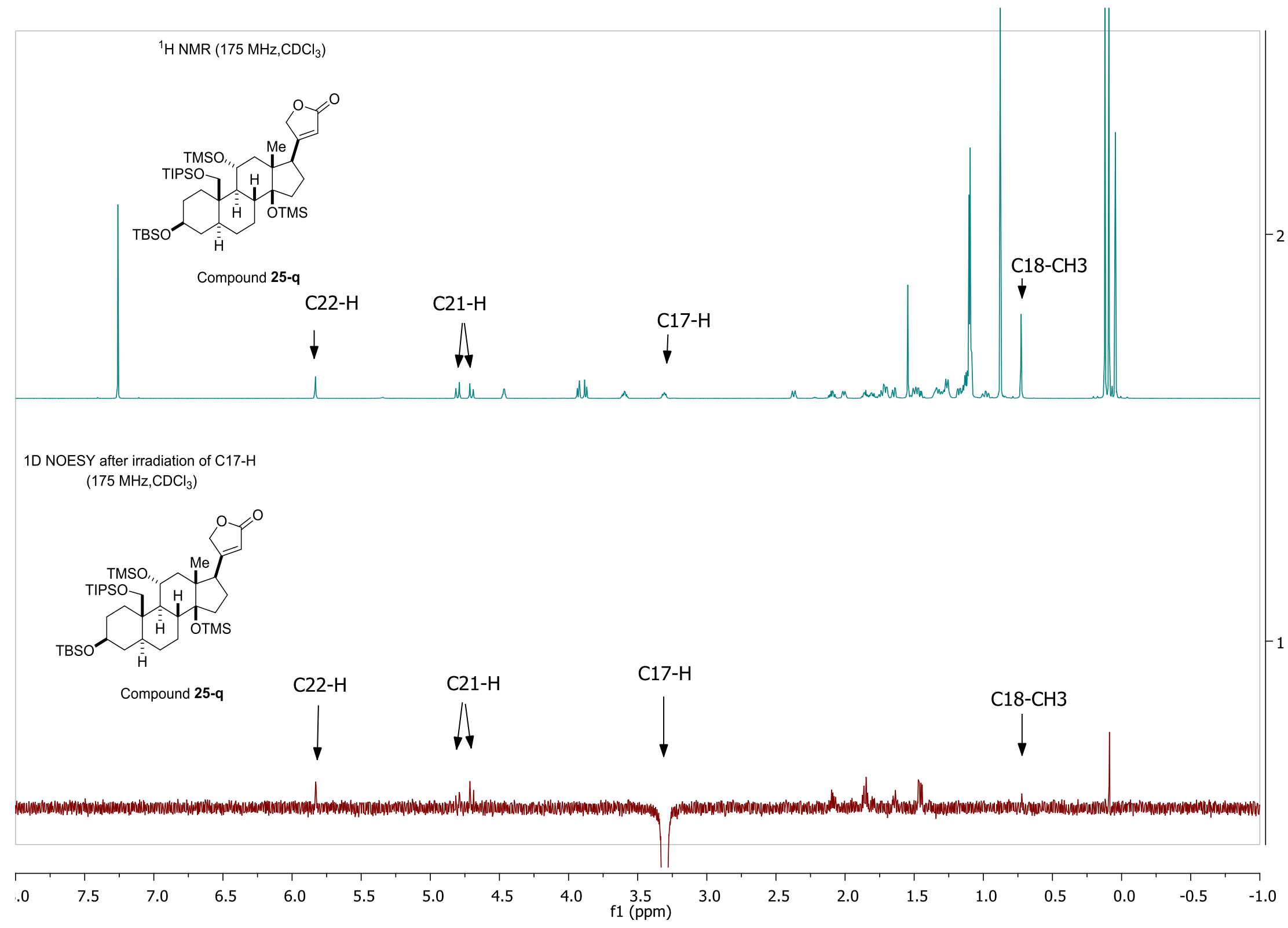


迎

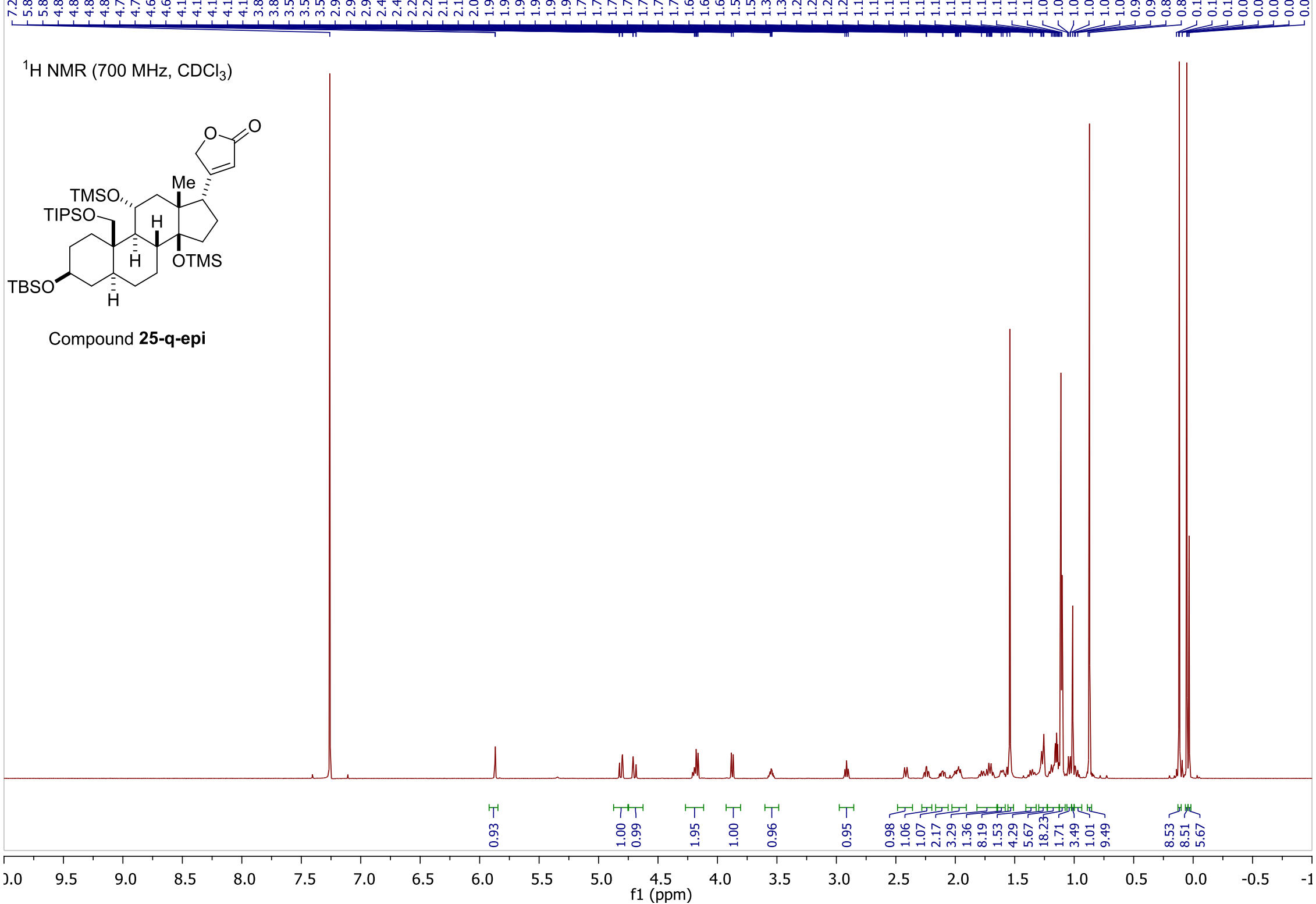




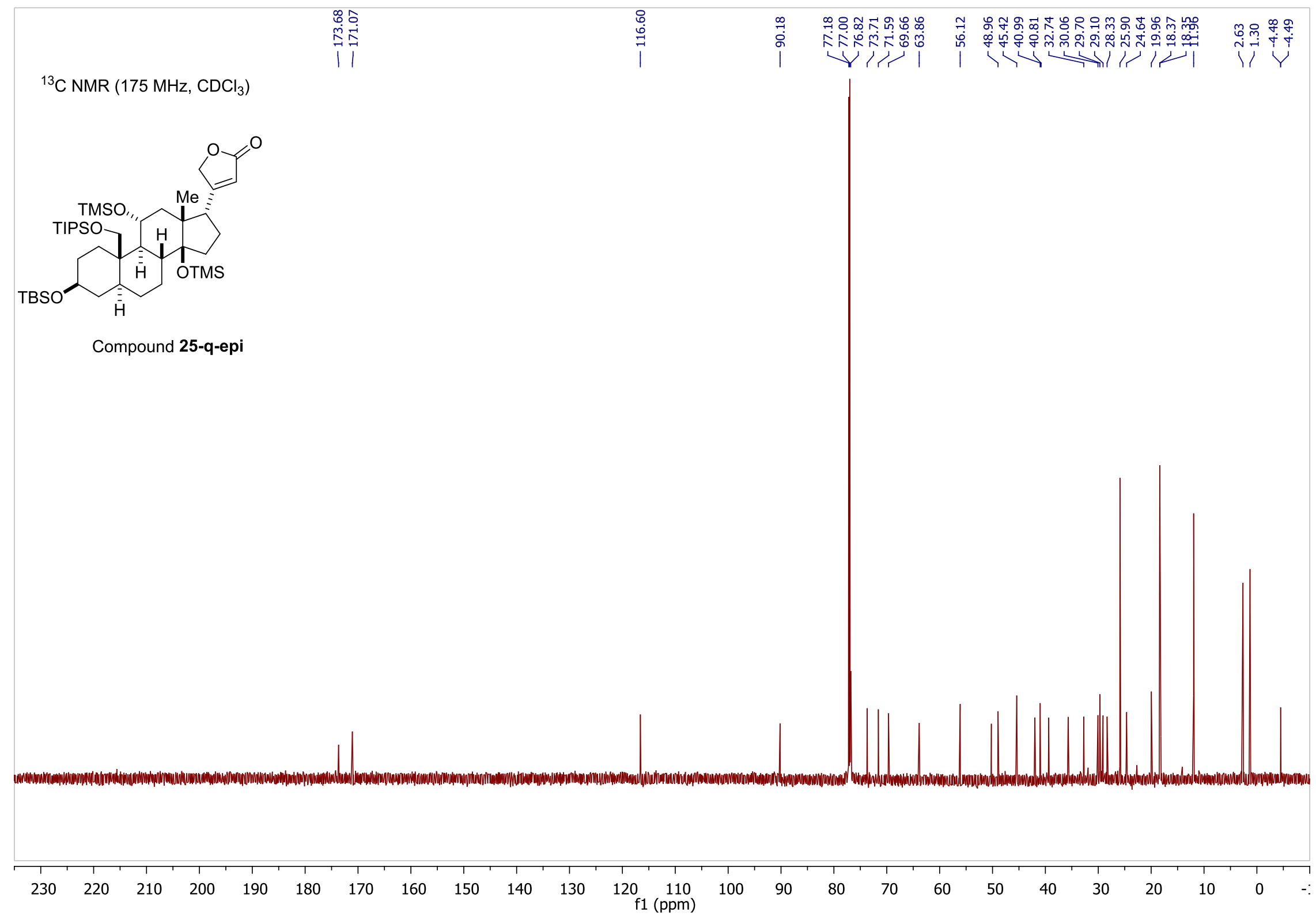


$\operatorname{cosy}\left(175 \mathrm{MHz}, \mathrm{CDCl}_{3}\right)$

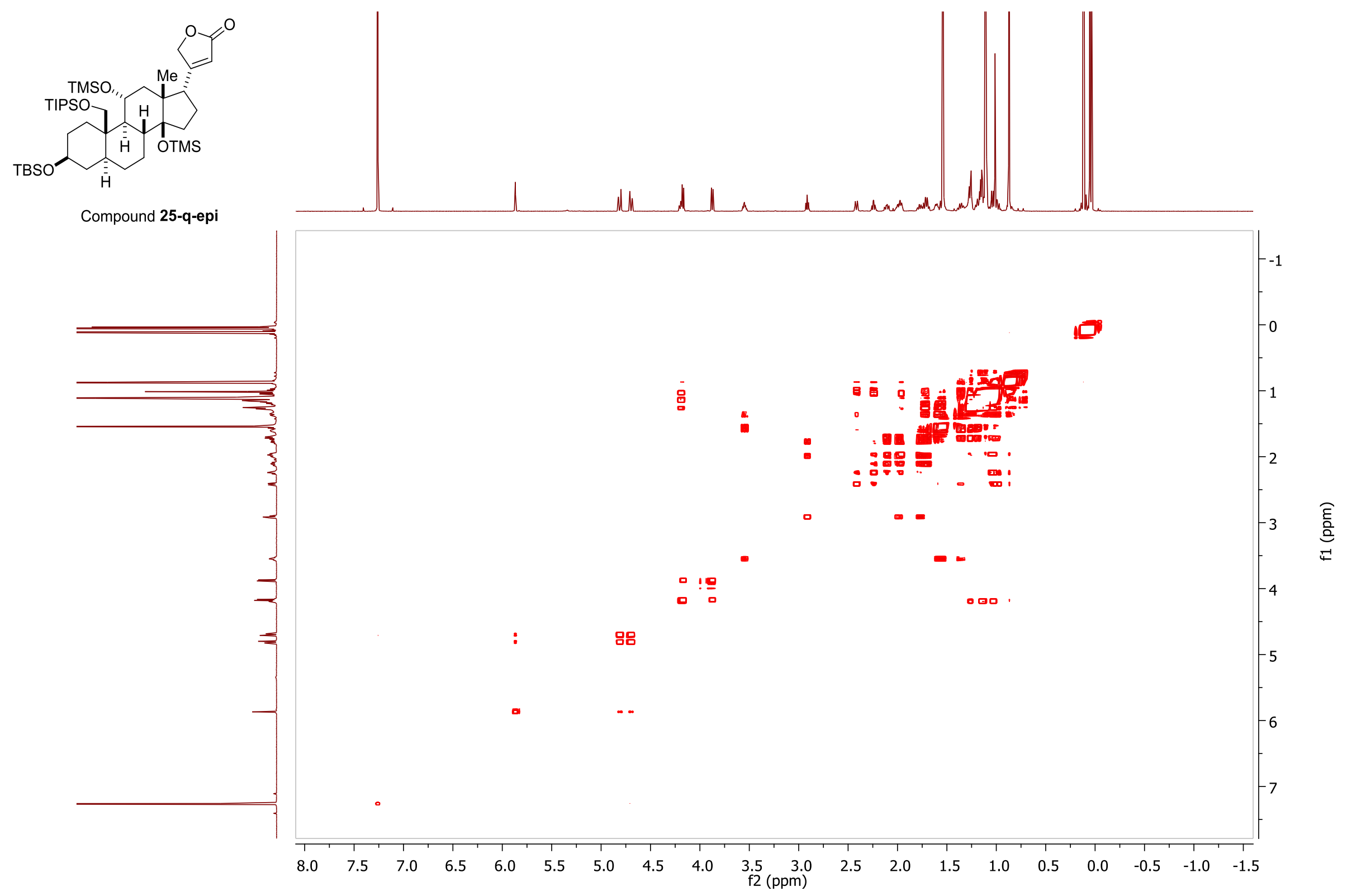


HSQC (175 MHz, $\mathrm{CDCl}_{3}$ )
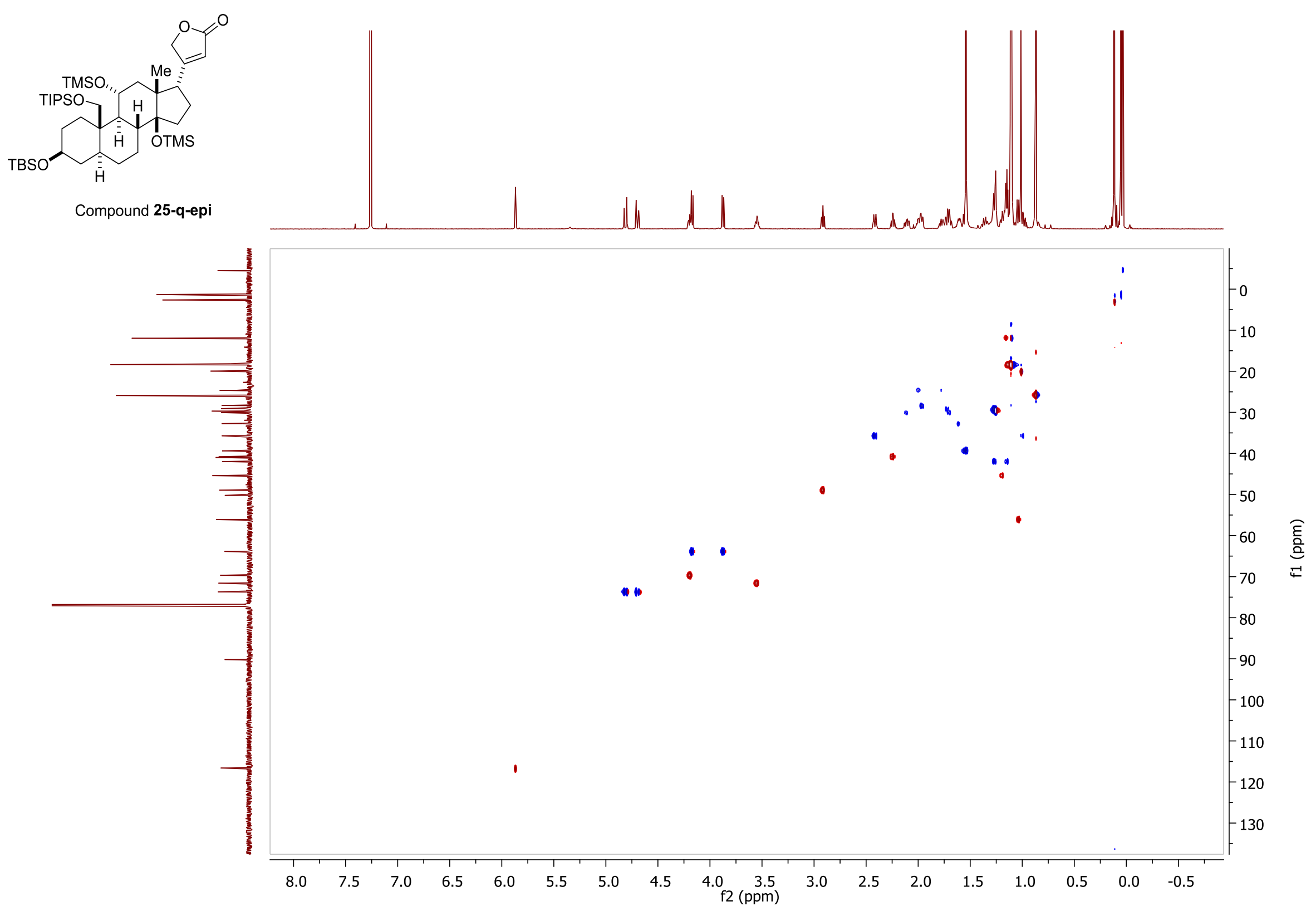


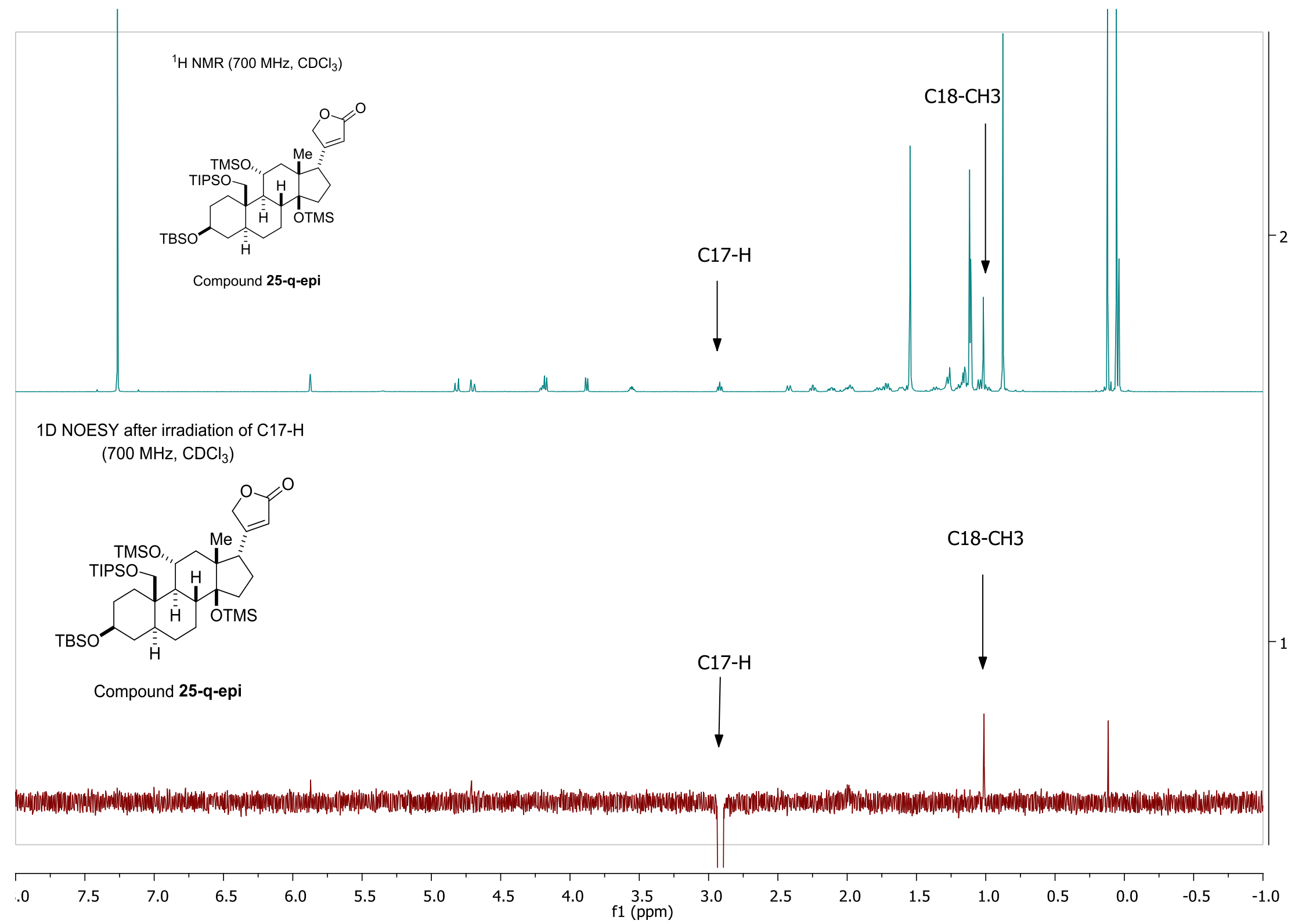




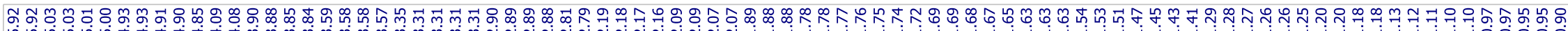

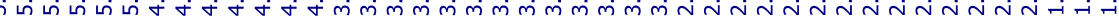

${ }^{1} \mathrm{H}$ NMR (700 MHz, CD ${ }_{3} \mathrm{OD}$ )

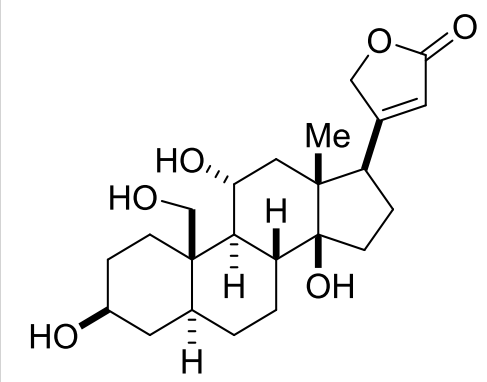

Trewianin aglycone (3)

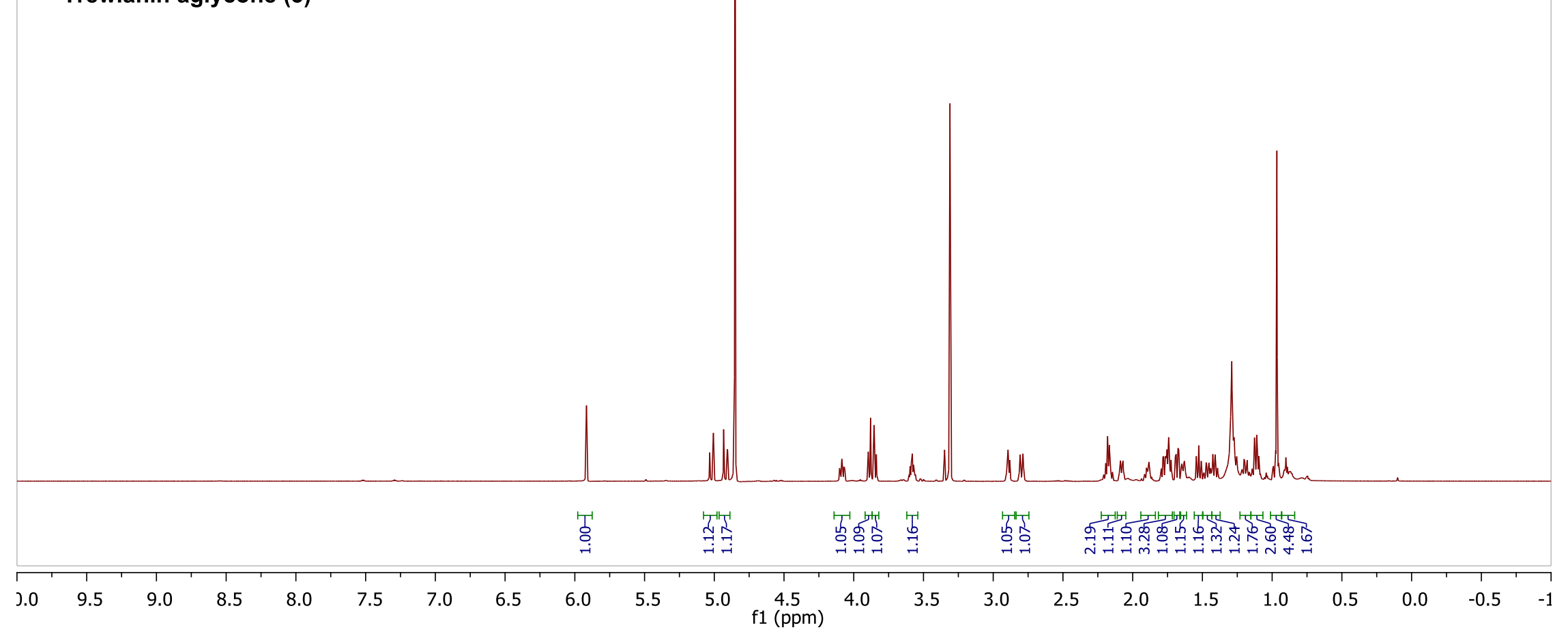



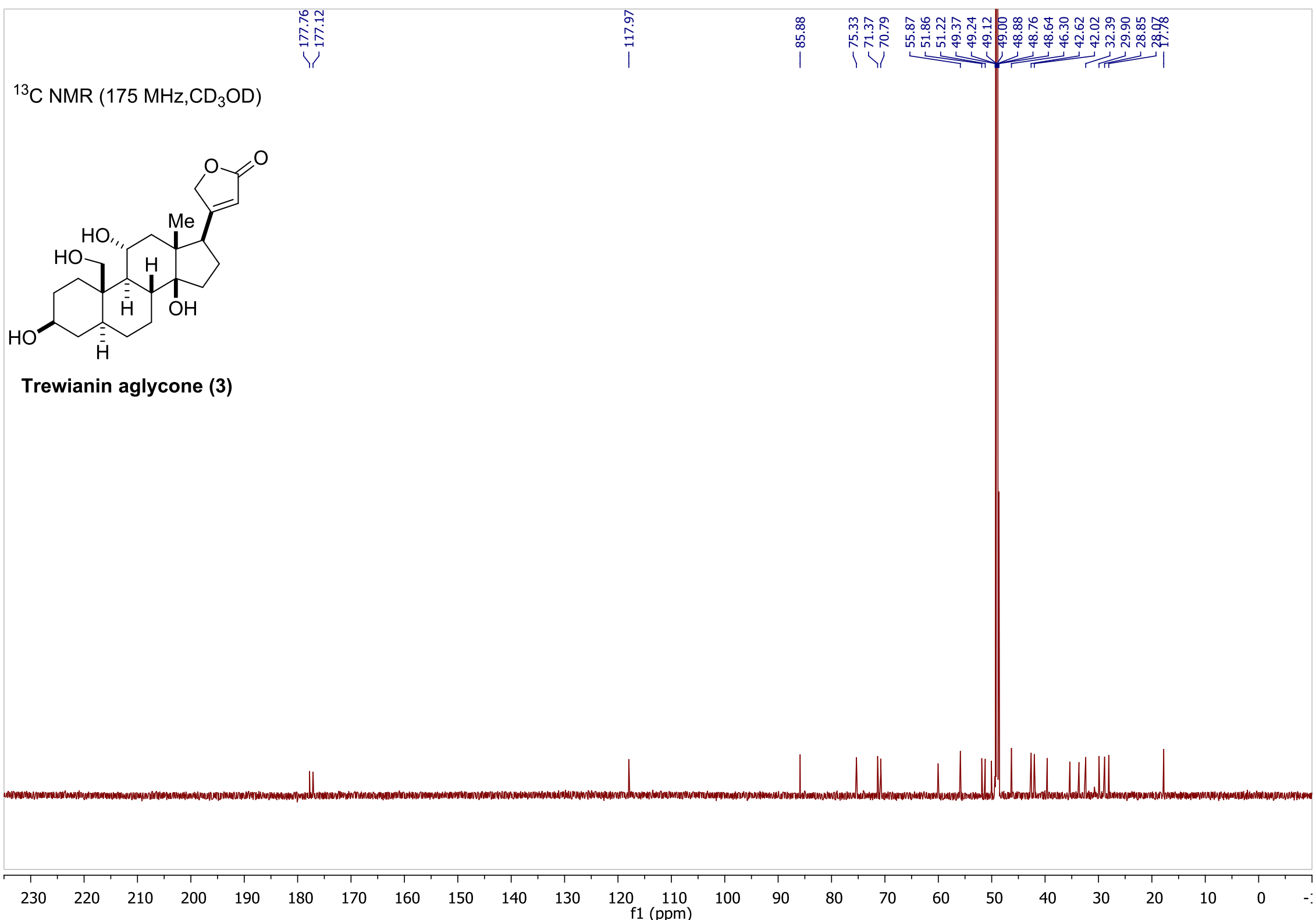


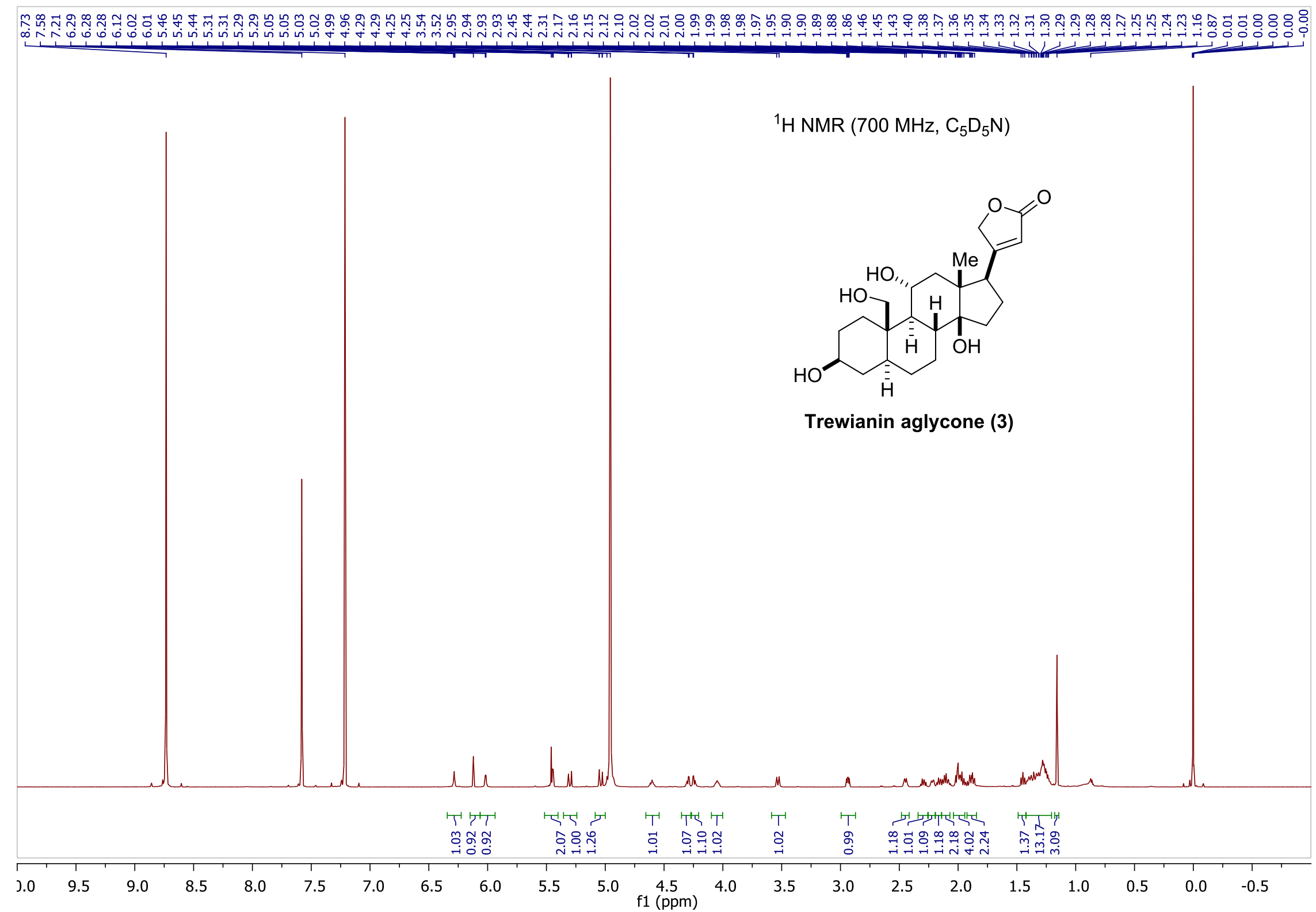




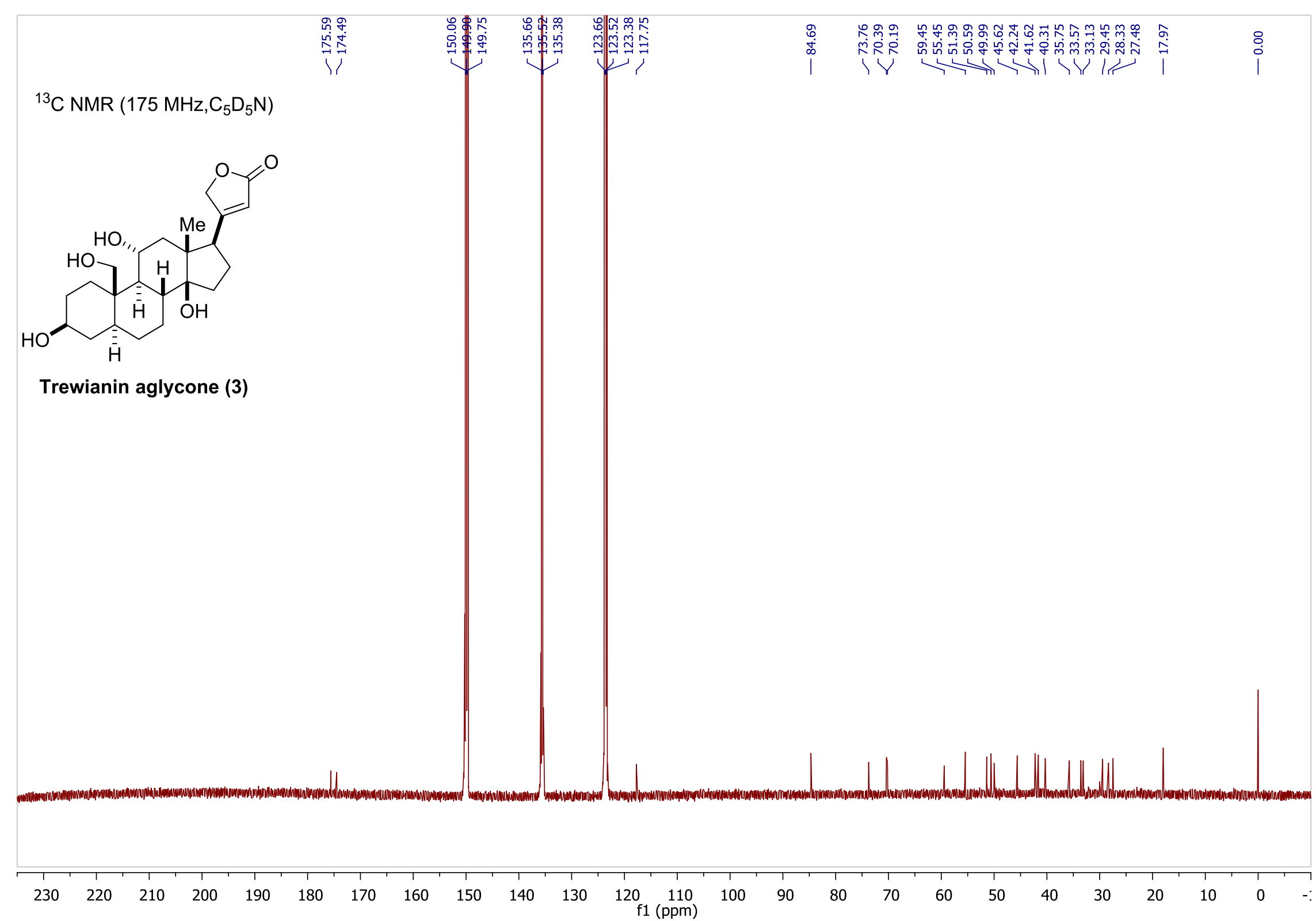


$\cos \left(175 \mathrm{MHz}, \mathrm{C}_{5} \mathrm{D}_{5} \mathrm{~N}\right)$
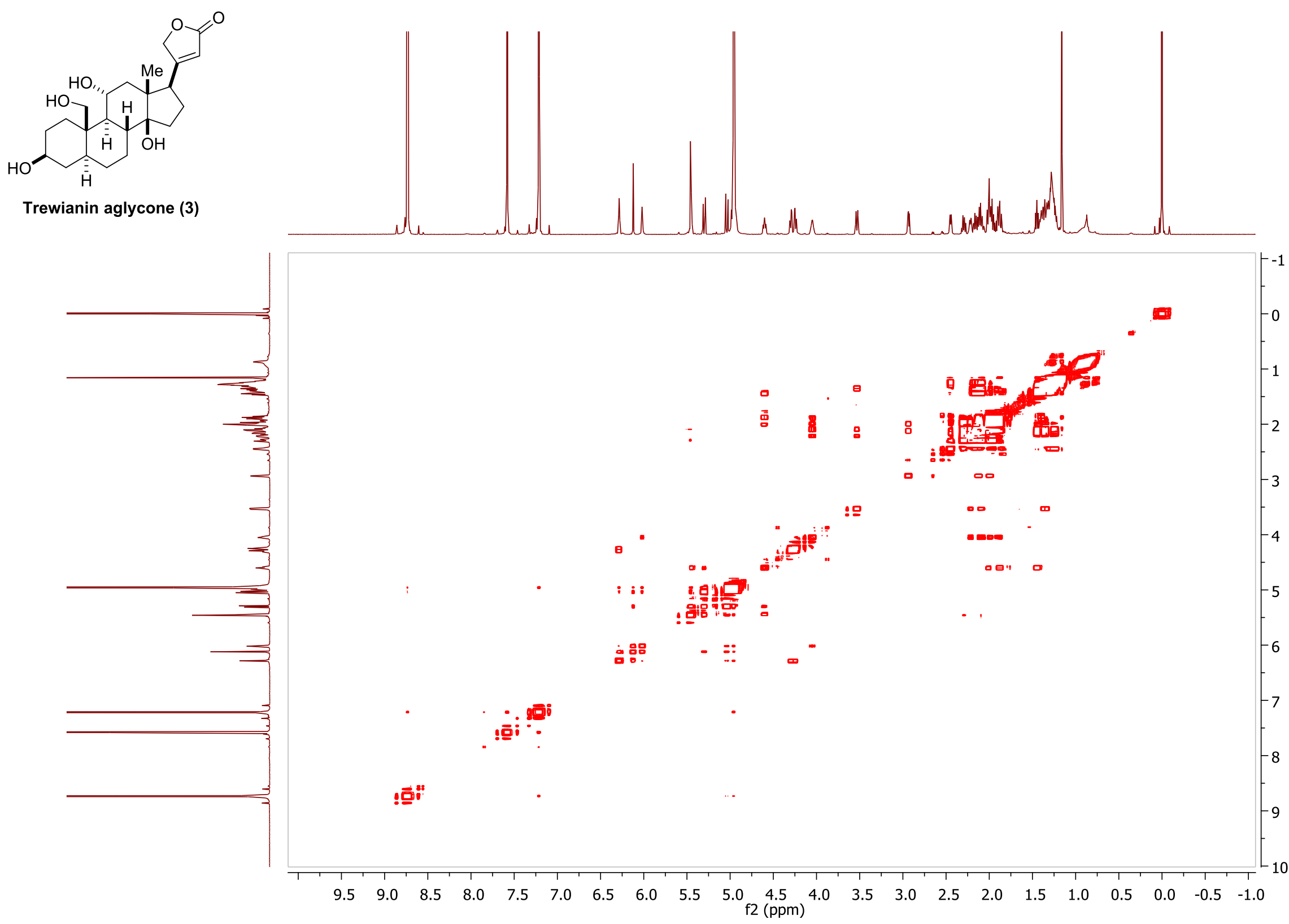
HSQC (175 MHz, $\left.\mathrm{C}_{5} \mathrm{D}_{5} \mathrm{~N}\right)$
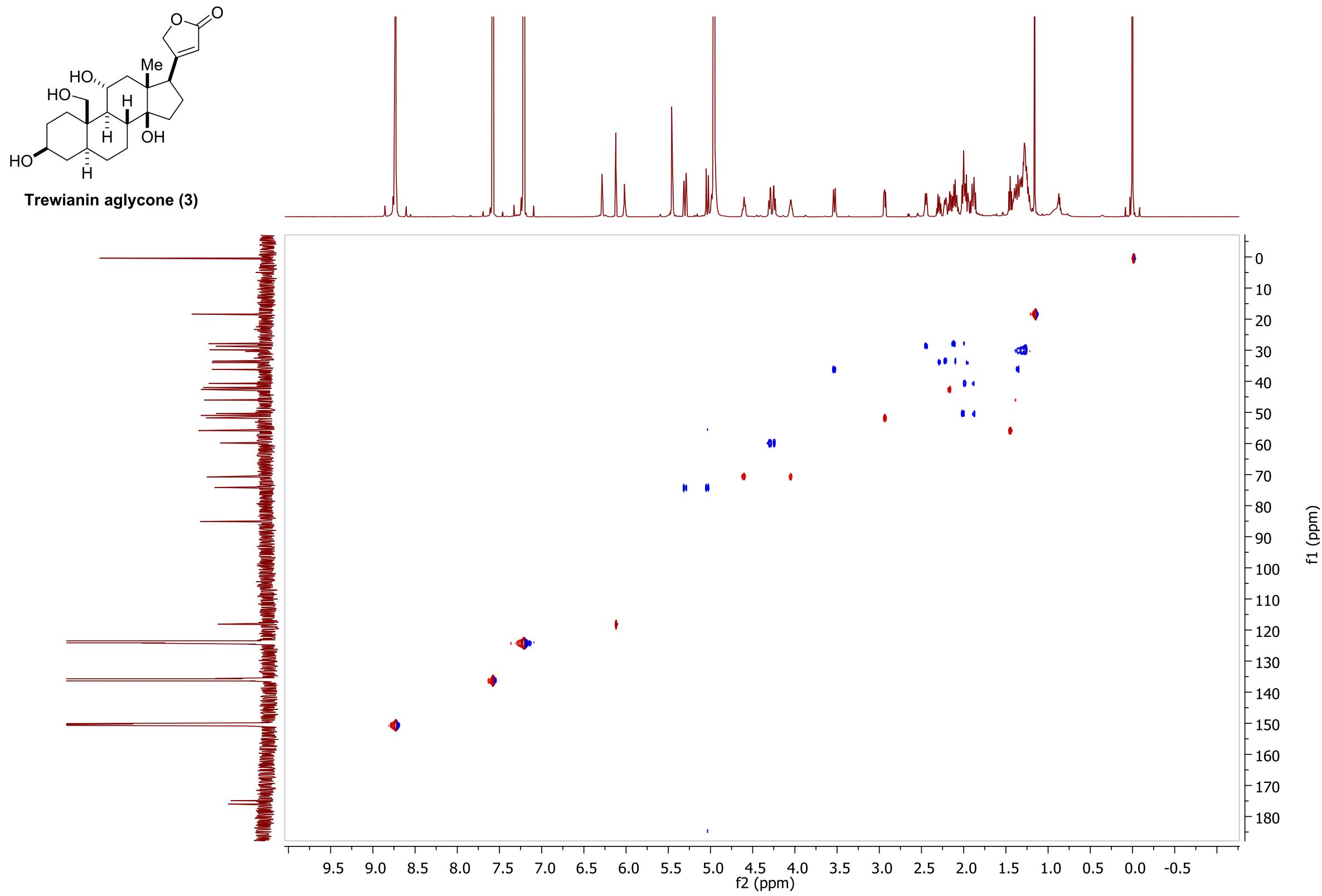
NOESY $\left(175 \mathrm{MHz}, \mathrm{C}_{5} \mathrm{D}_{5} \mathrm{~N}\right)$
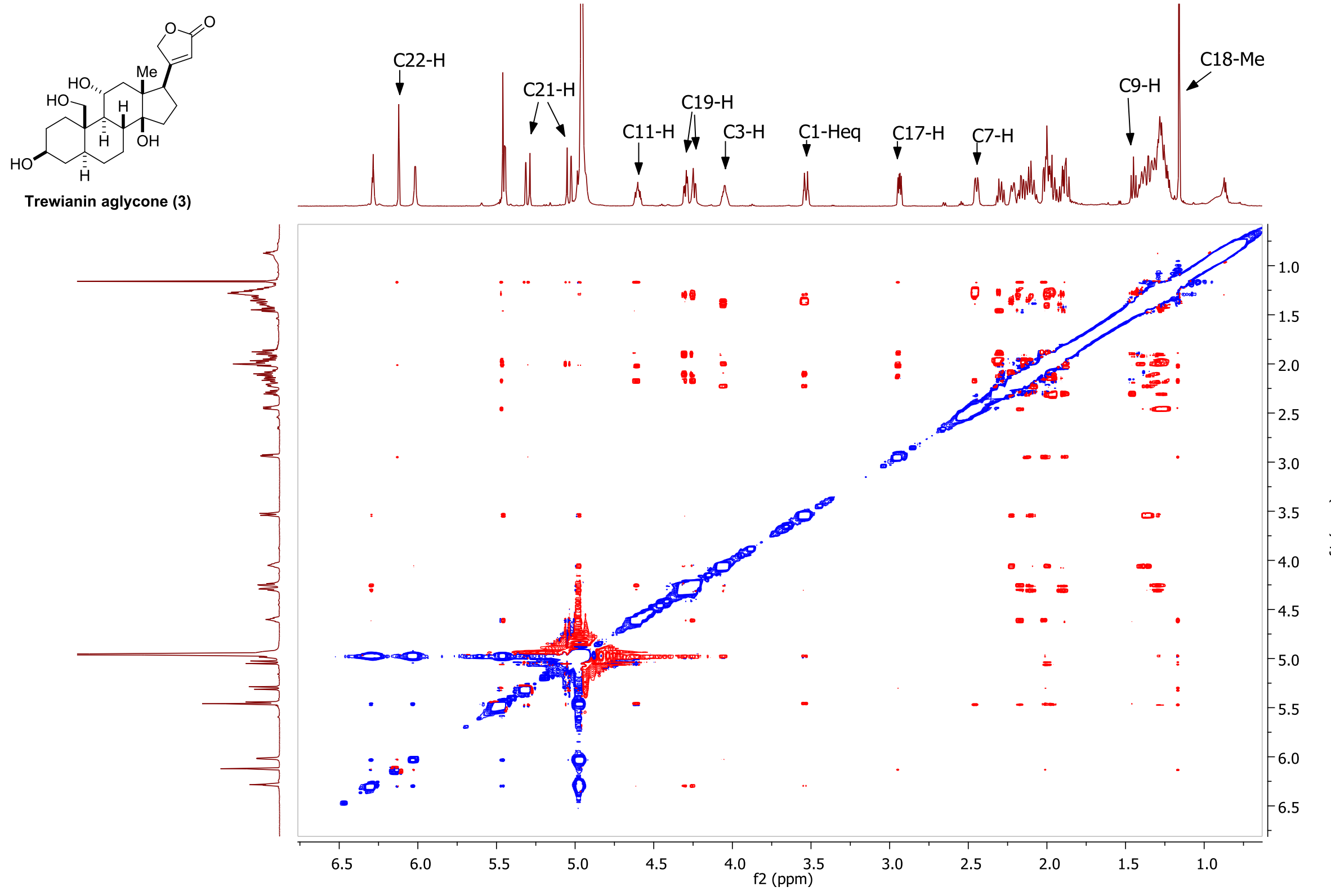
Sections of NOESY (175 MHz, $\mathrm{C}_{5} \mathrm{D}_{5} \mathrm{~N}$ ) for Trewianin aglycone (3)
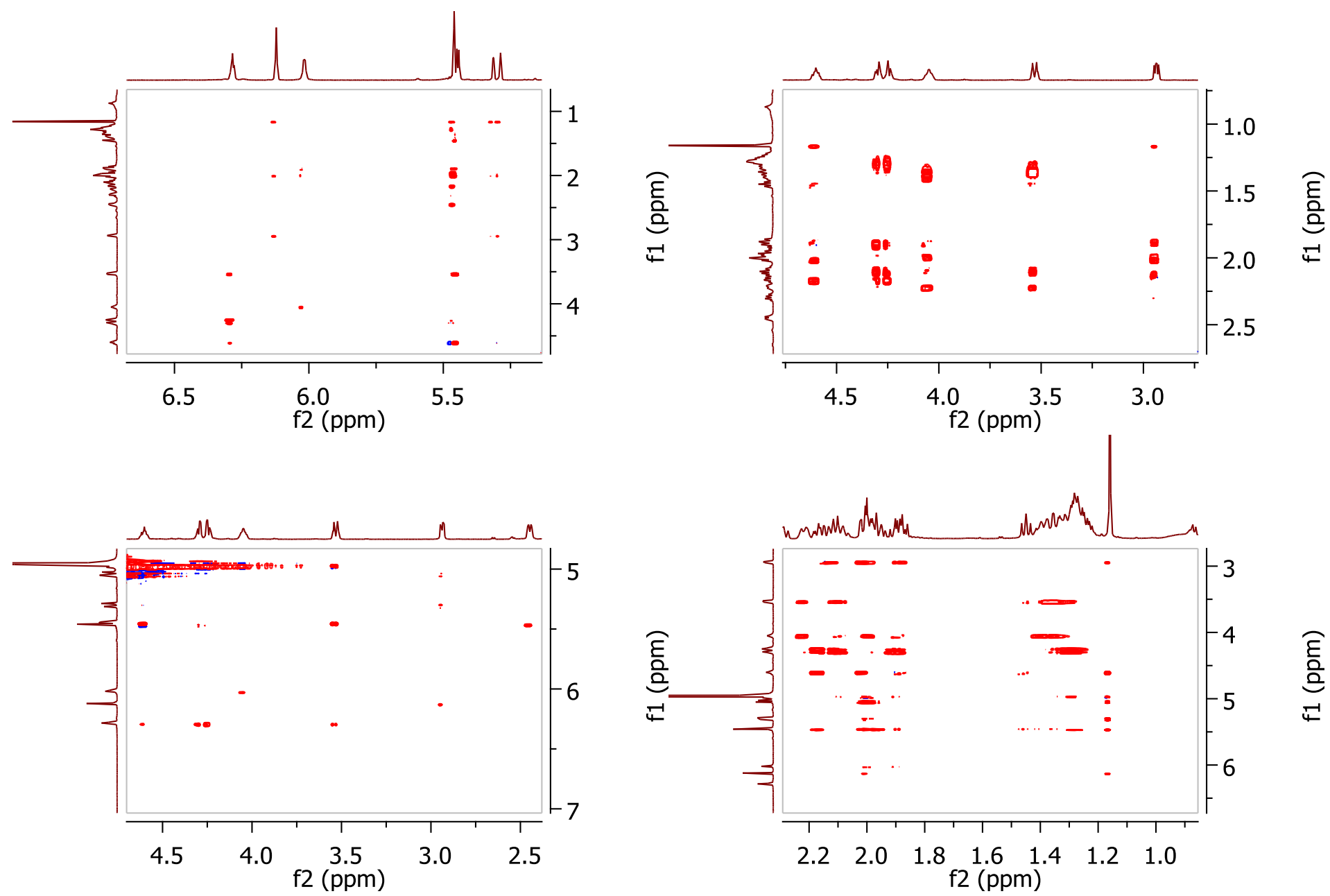


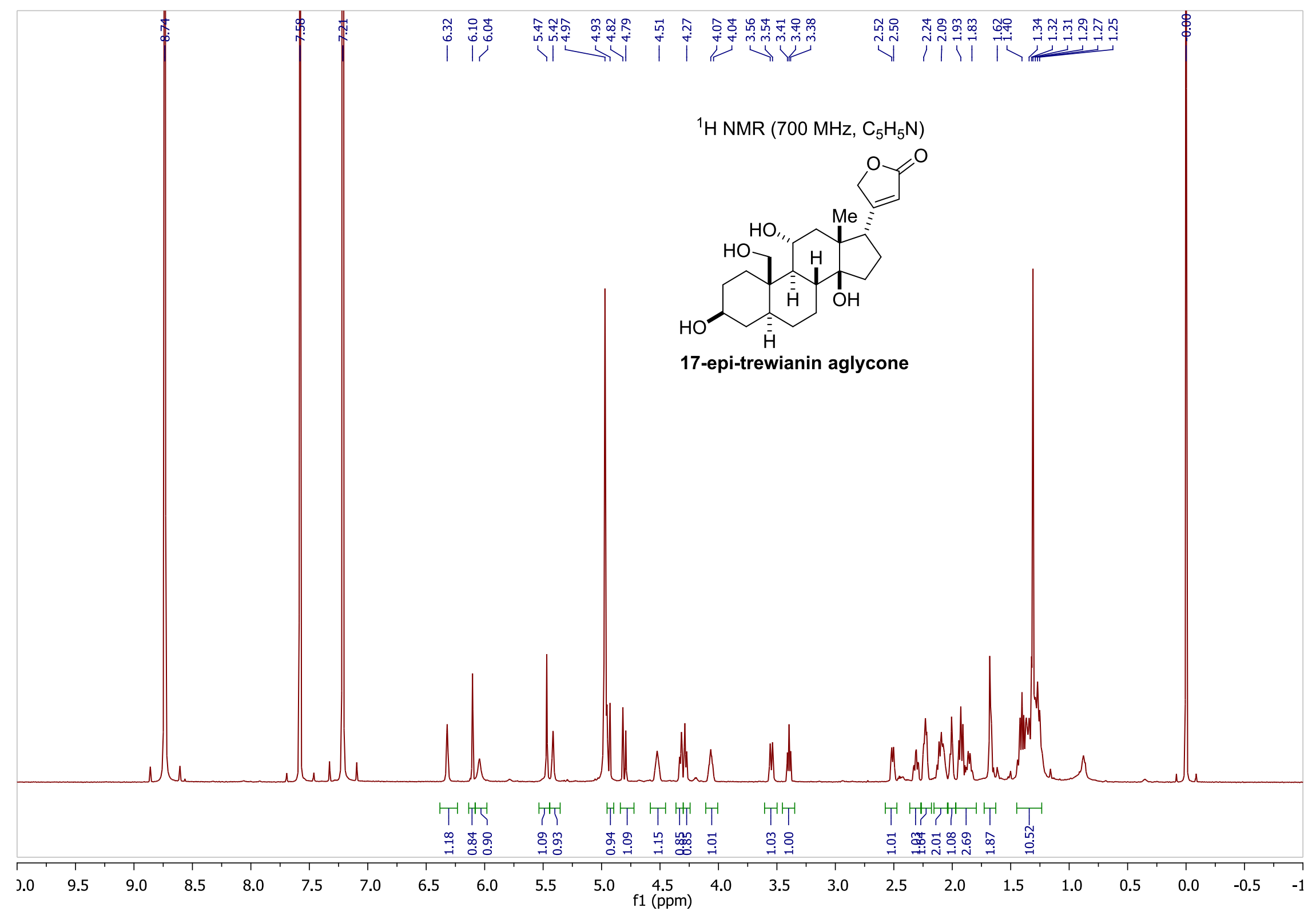



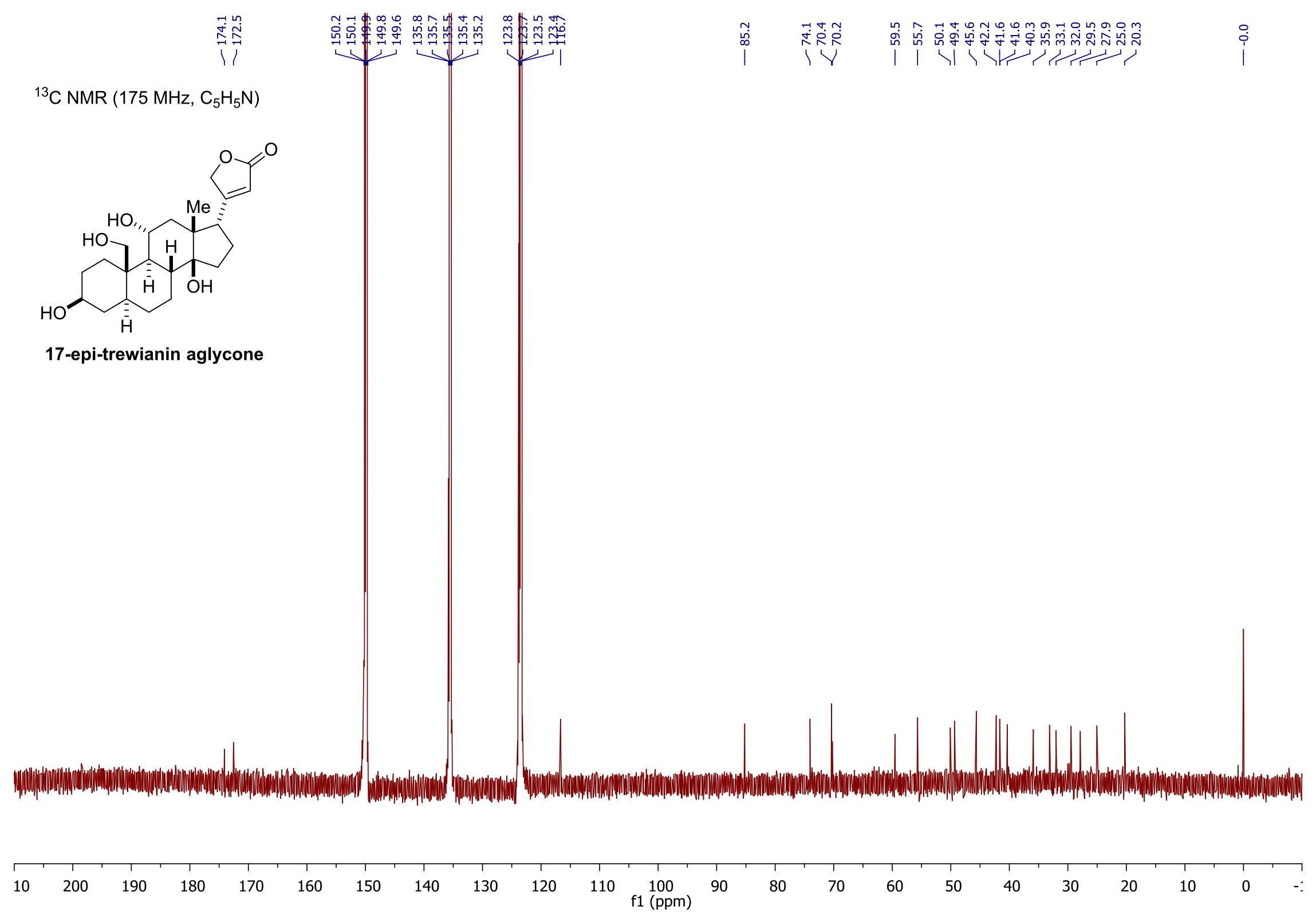


\begin{tabular}{|llll|}
\hline & \multicolumn{2}{c|}{ S A M PLE } & IN F O R M A T I O N \\
\hline \hline Sample Name: & racemic-Obz & Acquired By: & HPLCuser \\
Sample Type: & Unknown & Sample Set Name & racemic OBz \\
Vial: & 66 & Acq. Method Set: & adh 88 \\
Injection \#: & 1 & Processing Methoc & $\mathrm{n}$ \\
Injection Volume: & 15.00 ul & Channel Name: & $220.1 \mathrm{~nm}$ \\
Run Time: & 30.0 Minutes & Proc. Chnl. Descr.: & PDA $220.1 \mathrm{~nm}$ \\
& & \\
Date Acquired: & $3 / 19 / 20144: 44: 30$ PM EDT & & \\
Date Processed: & $2 / 9 / 20165: 40: 04$ PM EST & & \\
\hline
\end{tabular}

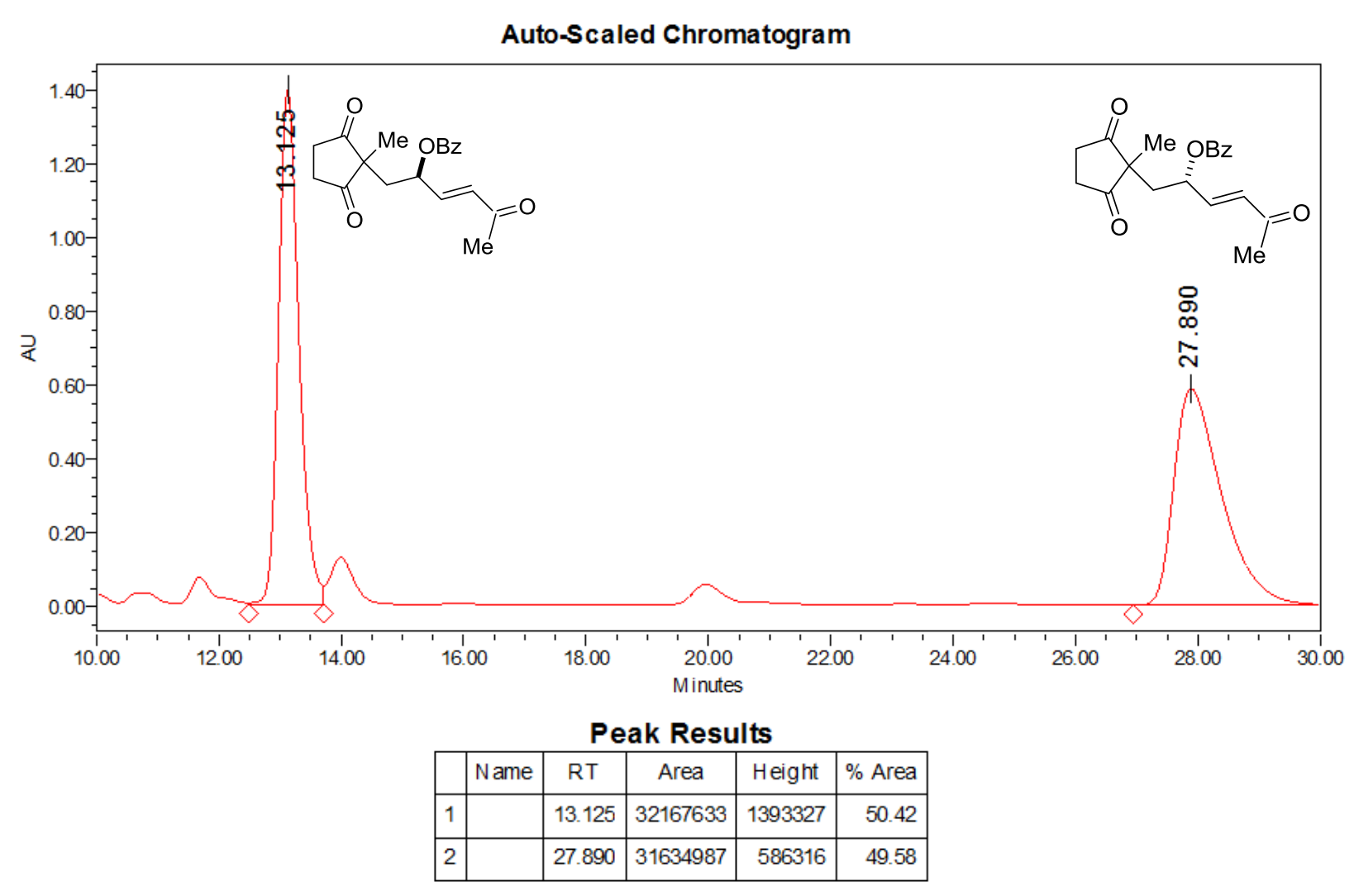




\begin{tabular}{|llll|}
\hline & \multicolumn{2}{c|}{ S A M PLE } & IN F O R M A T I O N \\
\hline \hline Sample Name: & wk-2-087 & Acquired By: & HPLCuser \\
Sample Type: & Unknown & Sample Set Name & wk 2 087 \\
Vial: & 66 & Acq. Method Set: & adh 88 \\
Injection \#: & 1 & Processing Methoc & enone chiral \\
Injection Volume: & $10.00 \mathrm{ul}$ & Channel Name: & $219.3 \mathrm{~nm}$ \\
- & & - &
\end{tabular}

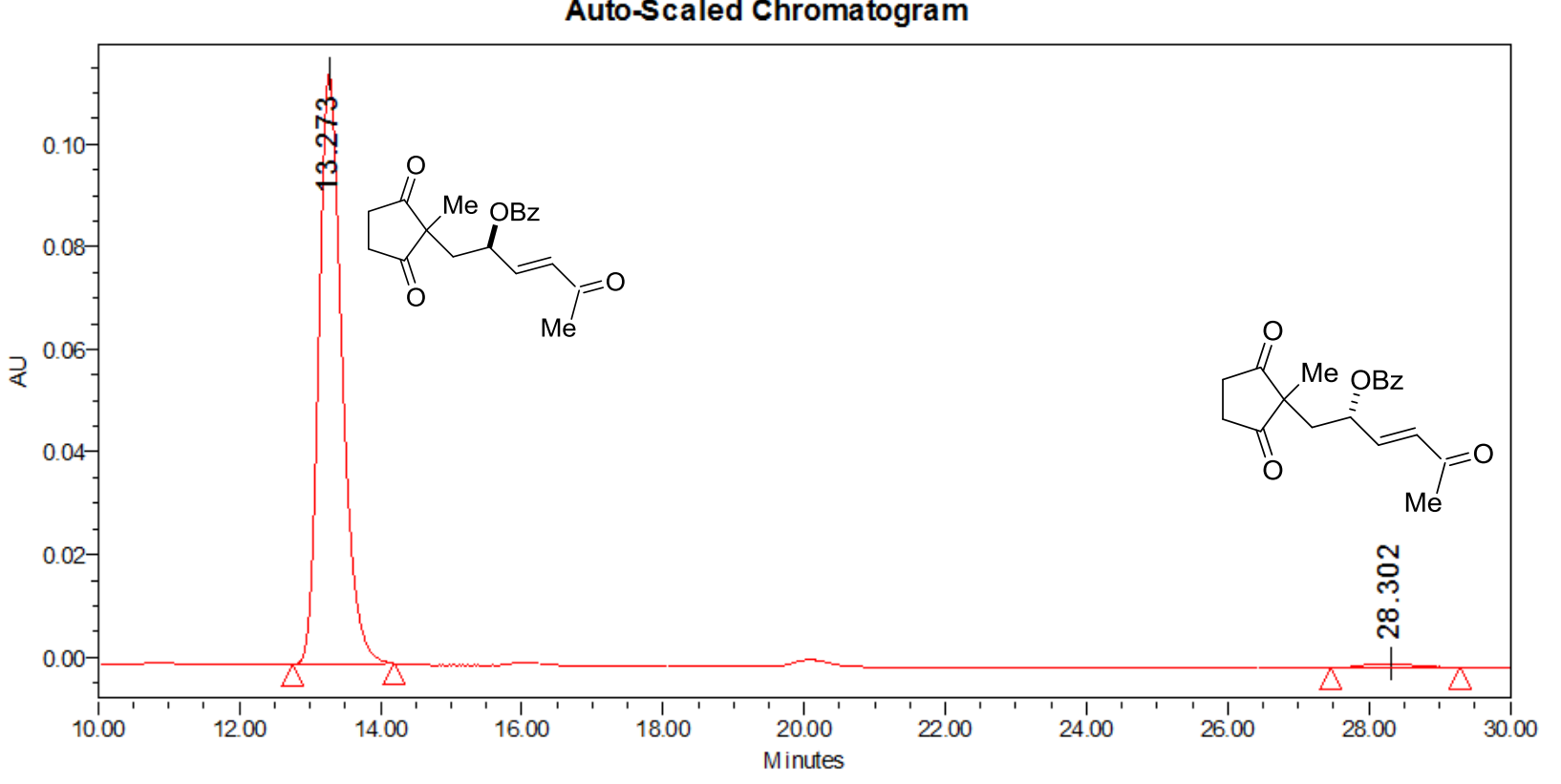

\section{Peak Results}

\begin{tabular}{|c|c|c|c|c|c|c|}
\hline & Name & RT & Area & Height & $\%$ Area & \multirow{3}{*}{$\begin{array}{l}\text { *The R-enantiomer of the enone is obtained by } \\
\text { using the D-proline-derived catalyst. }\end{array}$} \\
\hline 1 & & 13.273 & 2629917 & $11509 \mathrm{C}$ & 98.51 & \\
\hline 2 & & 28.302 & 39902 & 850 & 1.49 & \\
\hline
\end{tabular}




\section{References}

(1) Cichowicz, N. R.; Kaplan, W.; Khomutnyk, Y.; Bhattarai, B.; Sun, Z.; and Nagorny, P. J. Am. Chem. Soc. 2015137 (45), 14341.

(2) Mukai, K.; Urabe, D.; Kasuya, S.; Aoki, N.; and Inoue, M. Angew. Chem., Int. Ed. 2013, 52, 5300.

(3) Shilpi, J. A.; Gray, A. I.; Seidel, V. Fitoterapia 2010, 81, 536.

(4) Robien, W.; Kopp, B.; Schabl, D.; Schwarz, H. Progress In NMR Spectroscopy. 1987, 19, 131. 